\title{
AN ASSESSMENT OF MICROALGAL BIODIESEL WITH ACETONE, BUTANOL AND ETHANOL USING LIFE CYCLE ASSESSMENT (LCA) METHODOLOGY
}

by

\author{
Cherilyn Dignan \\ B. Eng Chemical Engineering, Royal Military College of Canada \\ Kingston, Ontario, Canada, 2008
}

A Thesis presented to Ryerson University in partial fulfillment of the requirements for the degree of

Master of Applied Science in the program of

Environmental Applied Science and Management

Toronto, Ontario, Canada, 2018

(C) Cherilyn Dignan, 2018 


\section{AUTHOR'S DECLARATION}

I hereby declare that I am the sole author of this thesis. This is a true copy of the thesis, including any required final revisions, as accepted by my examiners.

I authorize Ryerson University to lend this thesis to other institutions or individuals for the purpose of scholarly research.

I further authorize Ryerson University to reproduce this thesis by photocopying or by other means, in total or in part, at the request of other institutions or individuals for the purpose of scholarly research.

I understand that my thesis may be made electronically available to the public. 


\author{
ABSTRACT \\ AN ASSESSMENT OF MICROALGAL BIODIESEL WITH ACETONE, BUTANOL \\ AND ETHANOL USING LIFE CYCLE ASSESSMENT (LCA) METHODOLOGY \\ Master of Applied Science \\ 2018 \\ Cherilyn Dignan \\ Environmental Applied Science and Management \\ Ryerson University
}

Canada, as one of the largest producers and consumers of fossil fuels per capita on the planet, is attempting to reduce greenhouse gas (GHG) emissions. In order to accomplish this, fuel alternatives, such as biofuel, are required. Accordingly, this study uses LCA methodology to quantify the GHG impact of a unique biofuel production model. This unique model produces biodiesel (BD), acetone, butanol and ethanol (ABE) from microalgae and assesses the process GHG impact against other microalgal BD production processes.

This study's microalgal $\mathrm{BD}$ and $\mathrm{ABE}$ production process produces $76 \mathrm{kgCO}_{2} \mathrm{e}$ per functional unit, whereas other comparable microalgal BD production processes produce between 3.7 and 85 $\mathrm{kgCO}_{2} \mathrm{e}$. Overall, this study clarifies that without the development of versatile infrastructure to accommodate biofuel production, LCA studies will continue to find renewable fuel production processes net GHG positive for the simple reason that fossil resources are still the primary energy source. 


\section{ACKNOWLEDGEMENTS}

I would like to thank my family and friends for their support and encouragement. I may not have decided to pursue this area of study if they had not encouraged the interest they saw I had in environmental sustainability implementation.

I would like to thank Dr. Yaser Dahman for taking a chance on me.

Thank you to all the professors at Royal Roads University and Ryerson University who assisted and pushed me to improve my work and develop new perspectives. These individuals include Dr. Gillian Kerr, Dr. Glenn Brown, Dr. Lynda McCarthy, Dr. Seth Dworkin and my colleagues at Royal Roads and Ryerson Universities. 


\section{TABLE OF CONTENTS}

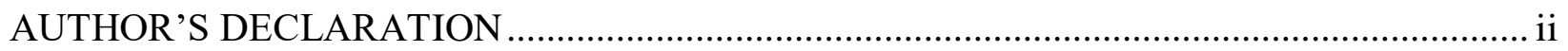

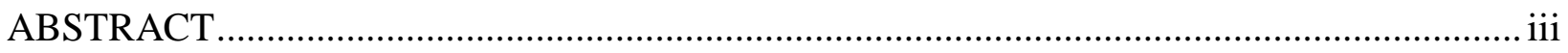

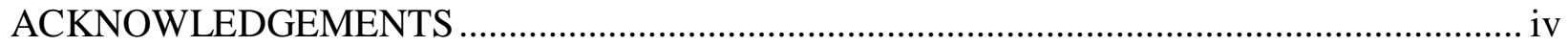

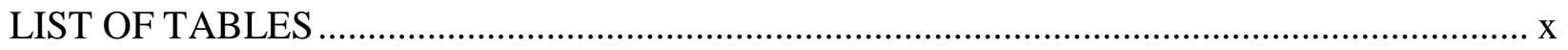

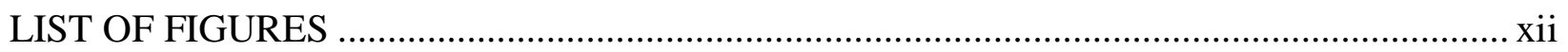

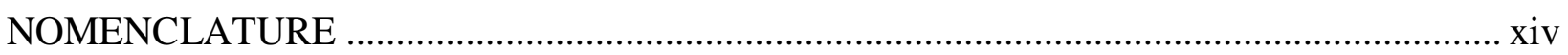

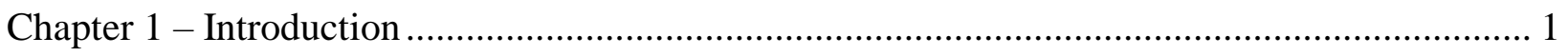

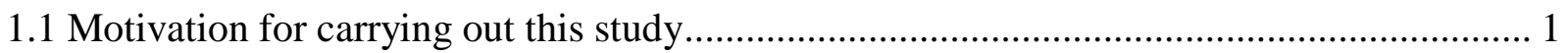

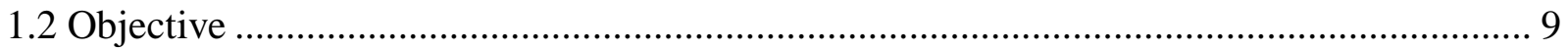

1.3 This study's intended application and audience …………................................................. 9

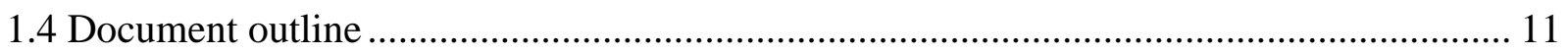

Chapter 2 - Biofuel production history and modern microalgal biofuel production advancements

2.1 Production and use of biofuel and their fossil counterparts ............................................. 13

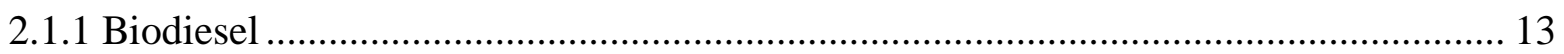

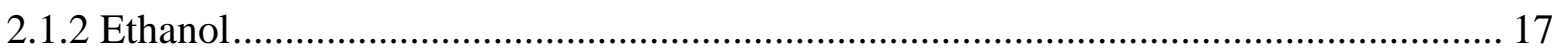

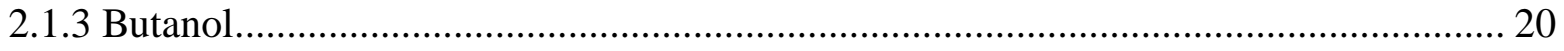

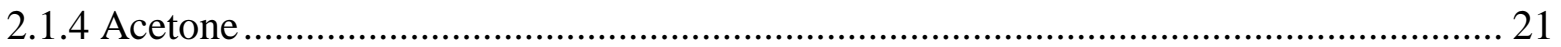

2.2 Biofuel impediments, government policy and government goals ..................................... 22

2.2.1 Biofuel production impediments ............................................................................ 22

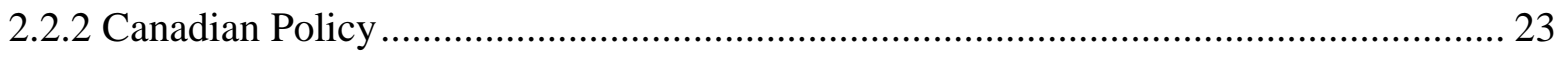

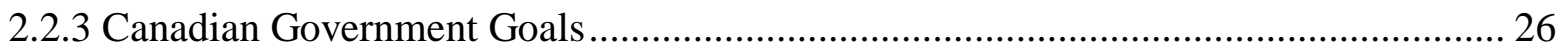

2.3 Microalgae as a source of feedstock for biodiesel and ABE............................................. 27

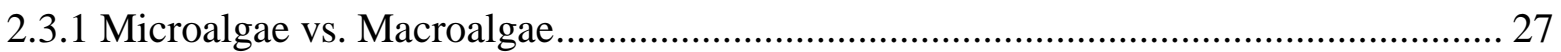

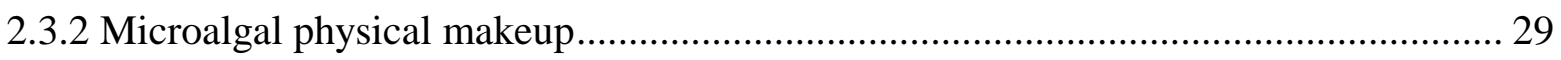

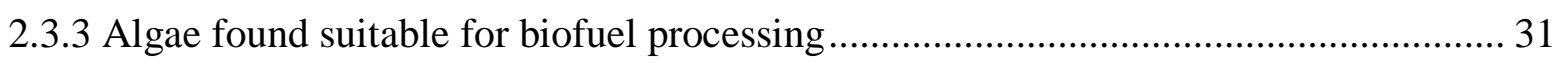

2.3.4 Enhancing microalgal growth while optimizing waste recycling and carbon

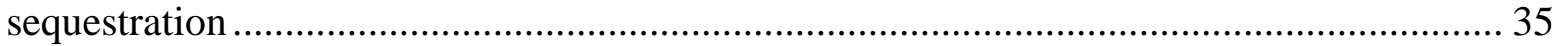

2.4 Microalgal Biofuel Processing Methods ....................................................................... 41

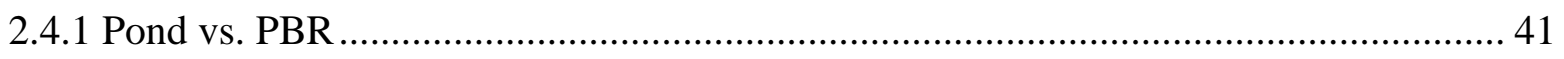

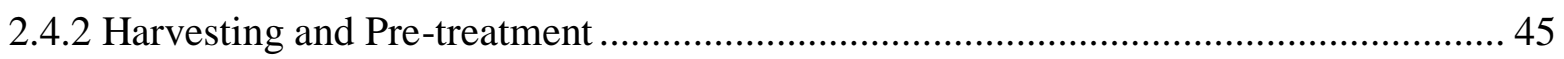


2.4.3 ABE production from lignocellulosic biomass ...................................................... 53

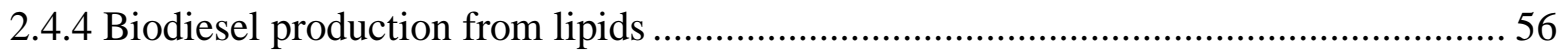

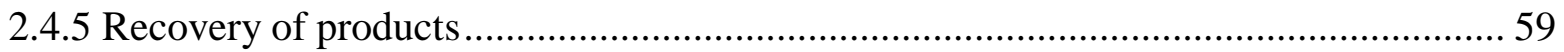

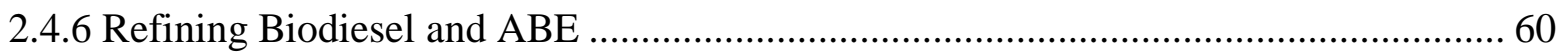

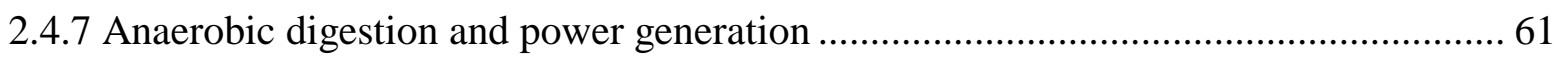

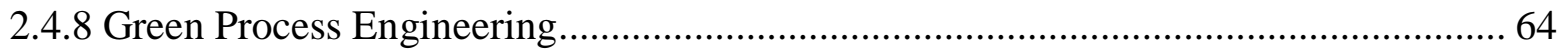

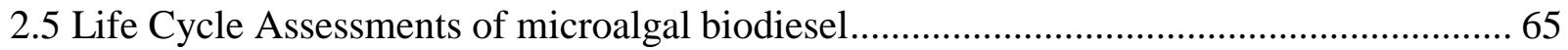

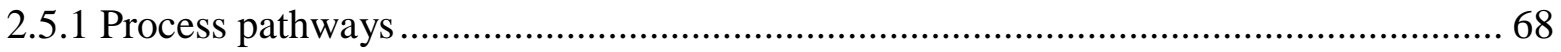

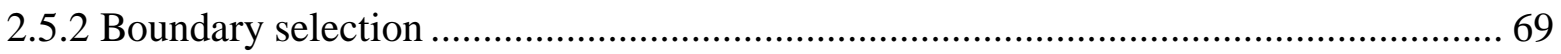

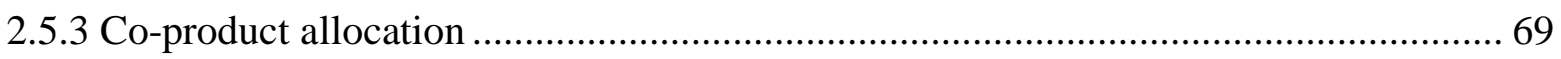

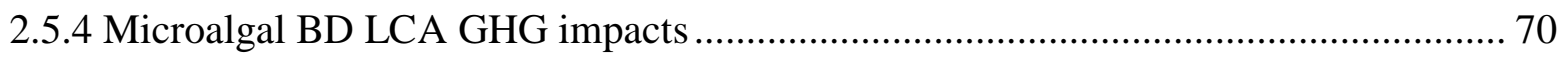

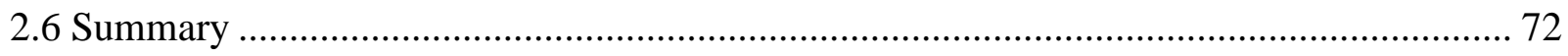

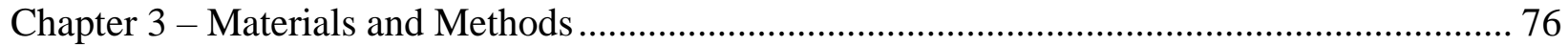

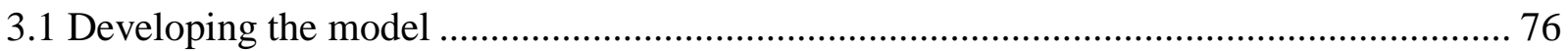

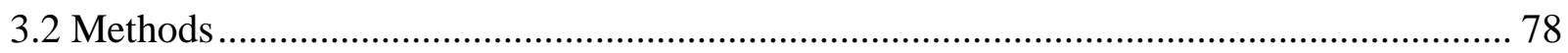

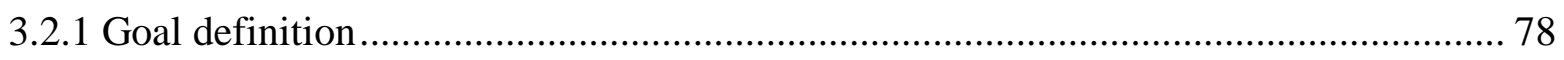

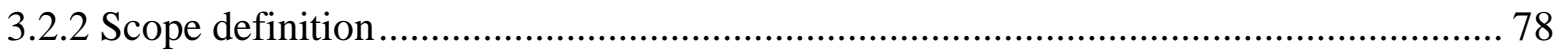

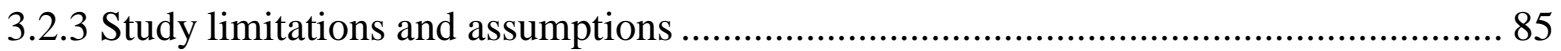

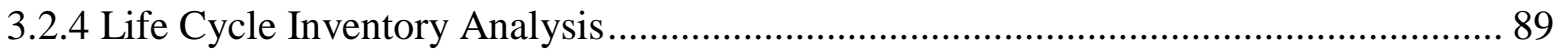

3.2.5 Life Cycle Impact Analysis Results ………………................................................. 94

Chapter 4 - Results, Discussion and Interpretation ................................................................ 99

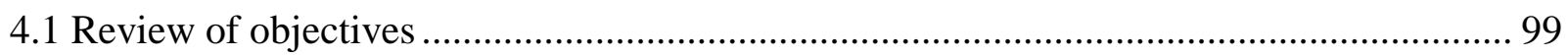

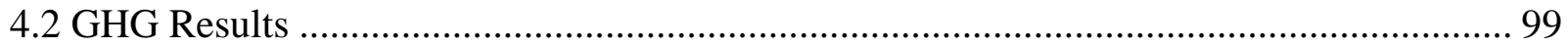

4.3 Comparison with other microalgal BD and co-product processes ................................... 100

4.3.1 Argonne National Laboratory study comparison ....................................................... 101

4.3.2 Biofuel output relative to microalgal biomass input ................................................. 106

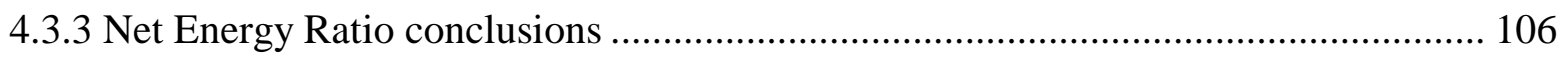

4.3.4 Feedstock parameter variations .................................................................................... 107

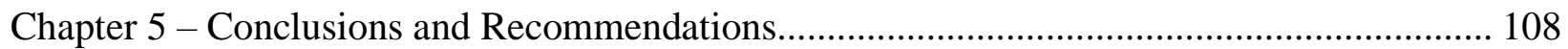

5.1 Model process adjustment....................................................................................... 108

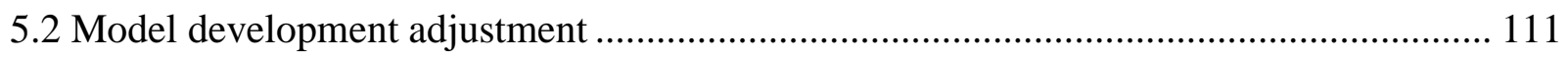

5.3 Model purpose adjustment ........................................................................................ 111 
5.4 Overall process conclusions 112

5.5 Anthropogenic system changes (The biorefinery concept and distribution system) 114

Appendices 117

Appendix A - Input/Output ( $\mathrm{IO}$ ) process parameters and calculations 117

A. 1 IO for PBR. 117

A.1.1 PBR 01 - Tubular photobioreactor (input \#2, 4, 5, 6, 7, 8, output \#1). 117 A.2 IO for Harvesting 124

A.2.1 FLC 01 - Flocculation (input \#3, 4, 5, output \#1) ……………………………..... 125

A.2.2 CEN 01 - Centrifuge to remove wastewater (input \#8, output \#3) ........................ 127

A.2.3 DRY 01 - Dryer to dry all microalgae (input \#11) .............................................. 128

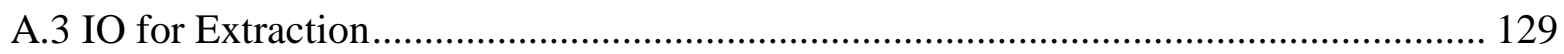

A.3.1 PUV 01 - Air swept pulveriser (input \#2) ......................................................... 129

A.3.2 CSTR 01 - Continuous Stirred Tank Reactor for separation mixing (input \#4, 5, 6, 7, output \#2,3) 130

A.3.3 CEN 02 - Centrifuge to separate lipids from rest of microalgal biomass (input \#12)

A.3.4 EVP 01 - Evaporate hexane from lipid stream (input \#15). 133

A.3.5 CND 01 - Condenser for hexane (input \#15) ...................................................... 135

A.3.6 EVP 07 - Evaporator for ethanol (input \#19) ………………………………....... 136

A.3.7 CND 07 - Condenser for ethanol (input \#21) ...................................................... 137

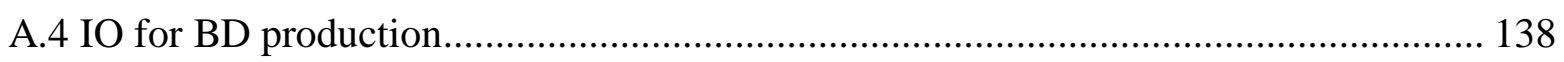

A.4.1 CMP 01 - Compressor for TG feed (input \#1, 3) ................................................. 139

A.4.2 CSTR 02 - Transesterification reaction (input \#6, output\# 3-8) ............................ 140

A.4.3 CLR 01 - Cooler for transesterification products (input\# 13)................................. 148

A.4.4 EVP 02 - Evaporate methanol (output\# 16) ........................................................ 148

A.4.5 CDN 02 - Condenser for methanol (input \#21, 22, output \#17) ............................. 149

A.4.6 CEN 03 - Centrifuge to separate glycerol and lipids (input \#29, output\#19) ........ 150

A.4.7 EVP 03 - Evaporate rest of methanol (input \#32, output \#24) .............................. 151

A.4.8 EVP 04 - Evaporate BD (input\# 38) ……………….................................... 152

A.4.9 CND 03 - Condenser to cool BD to liquid after EVP 05 (input\# 40) .................... 154

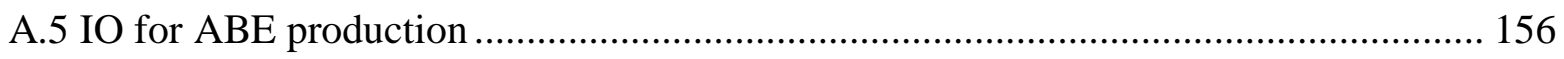


A.5.1 MXT 01 - Mixing sulfuric acid for pre-treatment of lignocellulosic \& protein biomass (input \#1, 2, 3, 4).

A.5.2 STX 01 - Heating of lignocellulosic and protein biomass (input \#7, output \#5, 6)

A.5.3 CLR 02 - Cool after pre-treatment (input \#12) ................................................ 160

A.5.4 MXT 02 - Neutralization tank (input \#14, 16, output \#15) ................................. 161

A.5.5 FRM 01 - Airlift Fermentation Reactor to produce alcohols (input \#19, 23, 24, 20,

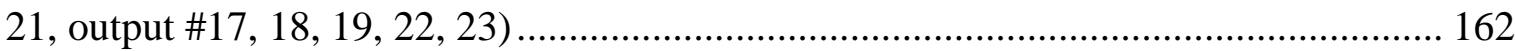

A.5.6 EVP 05 \& 06 - Evaporate bio-acetone and bio-ethanol (input \#28, 29, 30, 31) ... 167

A.5.7 CND 04 - Bio-acetone condenser (input \#33)................................................... 171

A.5.8 CND 05 - Bio-ethanol condenser (input \#38) .................................................. 172

A.6 IO for Anaerobic Digester and Co-generation facility ........................................ 173

A.6.1 AND 01 - Anaerobic digester (input \#4, output \#1, 2, 3)................................. 173

A.6.2 SRP 01 - Stripping biogas and fermenter gas (input 7) ................................. 177

A.6.3 COG 01 - Co-gereration facility for energy production (output \#6):.................... 177

Appendix B - Pump energy and refrigerant requirements............................................... 179

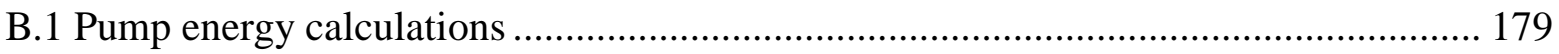

B.2 Refrigeration energy calculations ................................................................ 182

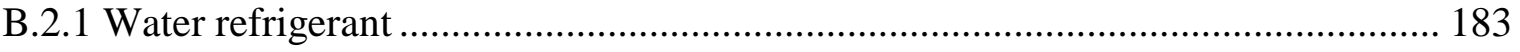

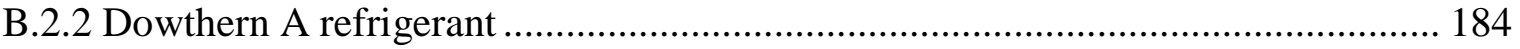

Appendix C - Sourcing materials (distance calculations and assumptions) ......................... 186

Appendix D - Life Cycle Impact Assessment Tables and Assumptions .............................. 189

D.1 PBR Life Cycle Impact Assessment Table, graph and assumptions ......................... 189

D.2 Harvest Life Cycle Impact Assessment Table, graph and assumptions ..................... 191

D.3 Separation Life Cycle Impact Assessment Table, graph and assumptions ................. 193

D.4 BD Life Cycle Impact Assessment Table, graph and assumptions ........................... 195

D.5 ABE Life Cycle Impact Assessment Table, graph and assumptions......................... 197

D.6 AD and Power Life Cycle Impact Assessment Table, graph and assumptions ........... 199

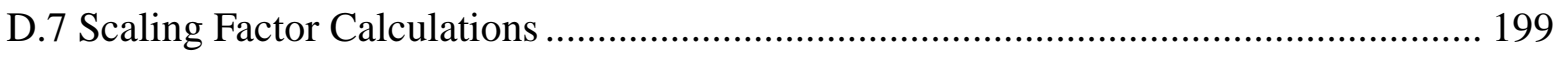

Appendix E - Comparison with other studies (calculations) ........................................... 200

E.1 Comparison with Frank et al. (2011) study calculations.......................................... 200

E.1.1 Difference between power requirements associated with cultivation + flocculation 
E.1.2 Difference in flocculation energy ........................................................................ 200

E.1.3 Difference in centrifuge energy calculations.................................................... 201

E.1.4 Incongruence between Frank et al. (2011)'s centrifuge energy requirements and gross energy use for remaining dewatering ................................................................ 201

E.1.5 Drying vs. wet processing (Homogenization) ………………………………..... 202

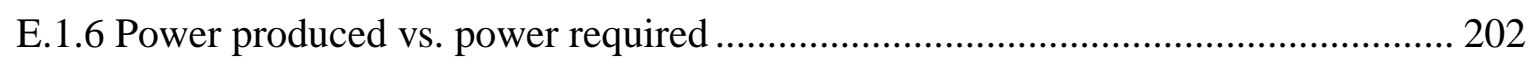

E.1.7 Different in total power requirements ............................................................. 202

E.1.8 Lignocellulosic power generation through digester instead of ABE...................... 203

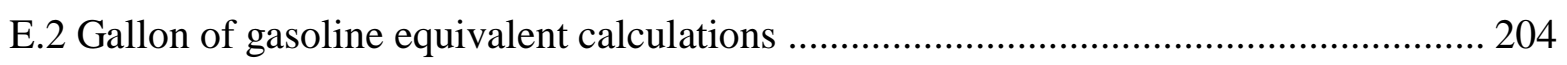

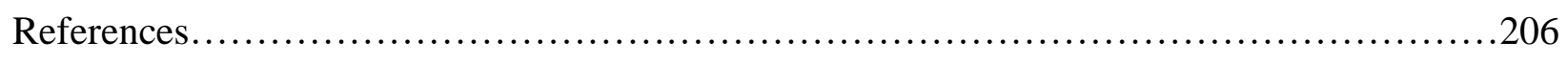




\section{LIST OF TABLES}

Table 2.1: Properties of algal biodiesel, fossil diesel and ASTM biodiesel standards ..............15

Table 2.2: Properties of (bio)ethanol, (bio)butanol and gasoline............................. 19

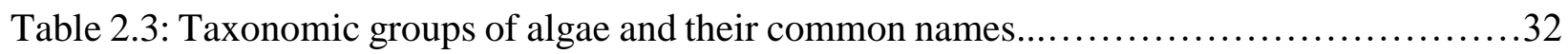

Table 2.4: Microalgal genus and their compositions...................................... 33

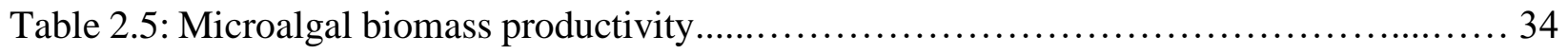

Table 2.6: Lipid content and FAME conversion for cyanobacteria genus........................35

Table 2.7: Microalgae subject to carbon dioxide emissions..................................

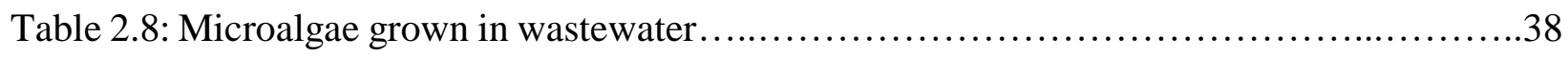

Table 2.9: Effect on algal Chlorella vulgaris culture with the addition of glycerol.................41

Table 2.10: Advantages and limitations of open ponds and photobioreactors....................42

Table 2.11: Biomass methane yield from anaerobic digestion..............................63

Table 2.12: Examples of key LCA variables associated with environmental impacts.............67

Table 2.13: Frank et al. (2013) GHG results for LE and HTL methods to produce RD.............68

Table 2.14: Studies that include GHG impact of microalgal BD and associated co-products, no infrastructure or maintenance included............................................. 74

Table 2.15: Low Sulfur Diesel life cycle GHG impacts.................................. 75

Table 3.1: Materials required for each stage of the microalgal $\mathrm{BD}$ and $\mathrm{ABE}$ production process

Table 3.2: Total climate change impact using three different functional units..................97

Table A.1: Cultivation input/output table............................................ 117

Table A.2: PBR nutrient sources.....................................................119

Table A.3: Harvesting input/output table.......................................... 124

Table A.4: Extraction input/output table......................................... 129

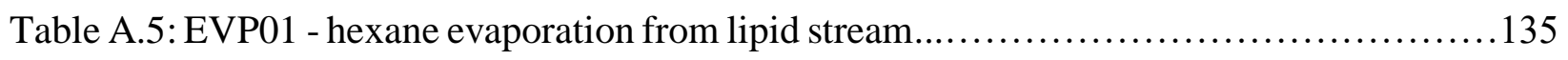

Table A.6: EVP07 - ethanol evaporation from lipid stream.............................. 137

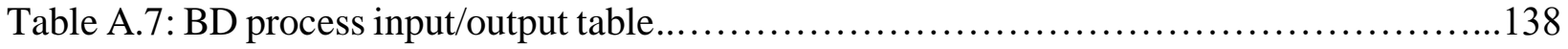


Table A.8: Properties of transesterification products................................... 143

Table A.9: Mass and molar flows of transesterification process.......................... 143

Table A.10: Heat capacity coefficients for liquids...................................... 144

Table A.11: Heat capacity coefficients for gases..................................... 144

Table A.12: Parameters associated with the evaporation of BD (FAME) from residue lipids.....152

Table A.13: Properties of BD stream for cooling purposes............................. 155

Table A.14: ABE process input/output table.......................................... 156

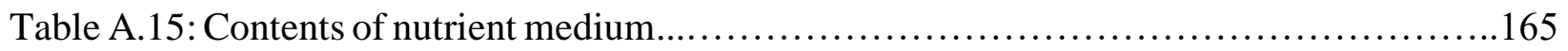

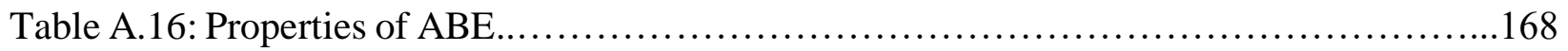

Table A.17: Properties of $\mathrm{ABE}$ in distillation column for increase in temperature from $45^{\circ} \mathrm{C}$ to

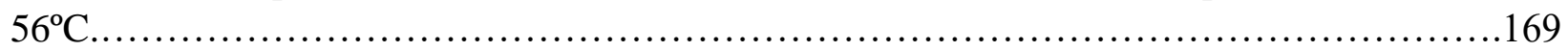

Table A.18: Properties of $\mathrm{ABE}$ in distillation column for increase in temperature from $56^{\circ} \mathrm{C}$ to

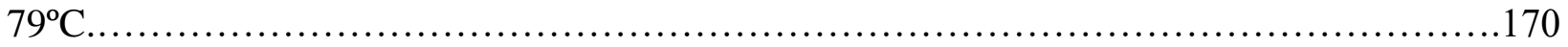

Table A.19: Anaerobic digestion and co-generation facility process input/output table...........173

Table B.1: List of pumps required - based on diagram in Figure 3.4 ....................... 179

Table B.2: Allocation of pump energy requirements by process.......................... 182

Table B.3: Heat energy and temperature delta required for calculating heating and cooling water capacity and power circulation requirements......................................... 183

Table B.4: Heating energy and temperature delta to calculate corresponding amount of Dowthern A and associated circulation power................................................. 184

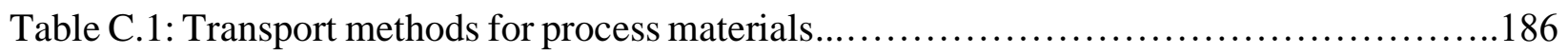

Table D.1: GHG impact associated with the PBR.................................... 189

Table D.2: GHG impact associated with the Harvest process: flocculation to dryer..............191

Table D.3: GHG impact associated with the Separation process: pulverizing to condensing of

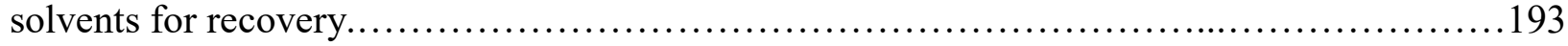

Table D.4: GHG impact associated with the Biodiesel production process.....................195

Table D.5: GHG impact for ABE production process................................. 197

Table D.6: GHG impact of AD and power production process............................. 199 


\section{LIST OF FIGURES}

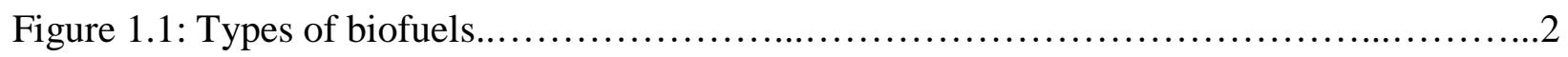

Figure 1.2: Biofuel production process pathways form microalgal biomass.....................6

Figure 1.3: Analogy of a bio-refinery approach for algal biomass with a petroleum refinery........7

Figure 2.1: Biodiesel and fossil diesel basic chemical formula..............................14

Figure 2.2: Chemical formulas of (bio)acetone (B), (bio)ethanol (A) and (bio)butanol (C)........18

Figure 2.3: Basic components (i.e., organelles) of a eukaryotic microalgae (left), Microsopic photo

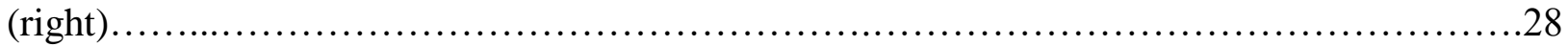

Figure 2.4: Macroalgae from plant to cellular level......................................29

Figure 2.5: Schematic of the location and structure of lignin (web), hemicellulose (casing) and cellulose (inside hemicellulose) in lignocellulosic biomass............................... 30

Figure 2.6: Triglyceride consisting of three fatty acids and glycerol backbone, carboxylic acid group is circled.................................................................

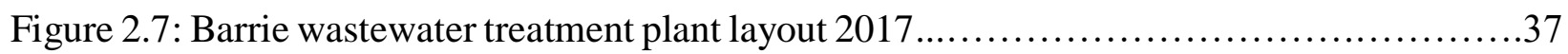

Figure 2.8: Typical horizontal tubular photobioreactor....................................44

Figure 2.9: Schematic of microalgal dewatering steps.................................. 45

Figure 2.10: Pre-treatment process schematic for microalgal biomass....................... 49

Figure 2.11: Scematic of solvent lipid-extraction mechanism for microalgal cells ...............51

Figure 2.12: Wastewater treatment process flow as it concerns microalgal biomass growth, nutrient

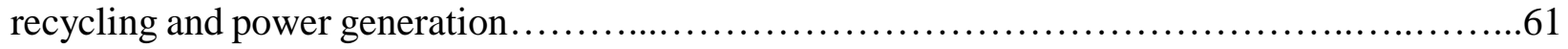

Figure 2.13: Life cycle of a product or process.....................................65

Figure 3.1: Conceptual model of carbon fixation using wastewater nutrients...................76

Figure 3.2: Cradle-to-grave scope of this study's operational microalgal biofuel life cycle and the cradle-to-grave scope of the operational fossil diesel's life cycle.............................84

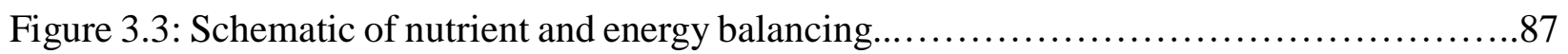

Figure 3.4: Microalgal BD and ABE process flow model ...............................90

Figure 3.5: Global warming potential contribution in $\mathrm{kgCO}_{2} \mathrm{e}$ for this study's microalgal BD and $\mathrm{ABE}$ production process based on $100 \mathrm{~km}$ driven in a compact diesel vehicle..................98

Figure 4.1: GHG impact distribution of NG, material and power per model stage..............100 
Figure 4.2: Percent Average Ontario Electricity Generation by Source in 2018 .103

Figure 4.3: Energy requirements (input) and outputs of this study's process on a per hour basis

Figure 5.1: Canadian percent electrical power generation sources in 2014 ('14) and predicted for 2040 ('40). 114

Figure A.1: Falling film evaporator.

Figure D.1: Global Warming Potential of PBR process requirements.......................... 190

Figure D.2: Global Warming Potential of Harvesting process requirements....................192

Figure D.3: Global Warming Potential of Separation process requirements................... 194

Figure D.4: Global Warming Potential of BD process requirements ......................... 196

Figure D.5: Global Warming Potential of ABE process requirements......................... 198 


\section{NOMENCLATURE}

(bio)acetone - referring to both bio-acetone and acetone

(bio)butanol - referring to both bio-butanol and butanol

(bio)ethanol - referring to both bio-ethanol and ethanol

$\mathrm{ABE}$ - bio-acetone, bio-butanol and bio-ethanol

AD - Anaerobic Digestion

ASP - Aquatic Species Program

BD - Biodiesel

Bio-acetone - Acetone produced by fermentation

Bio-butanol - Butanol produced by fermentation

Bio-ethanol - Ethanol produced by fermentation

BMP - Biological methane potential

BOD - Biological oxygen demand

CCAP - Climate Change Action Plan

CEN - Centrifuge

CHP - Combined Heat and Power

CIE - Combustion Ignition Engine

CIE - Compression Ignition Engine

CMP - Compression

$\mathrm{CN}$ - Cetane Number

$\mathrm{CO}$ - Carbon Monoxide

COD - Chemical Oxygen Demand

CSTR - Continuous Stirred Tank Reactor

DAF - Dissolved Air Floatation

DAP - Di-Ammonium Phosphate

DG - Diglyceride Fatty Acid

DRY - Dryer

EMS - Environmental Management System

EROI - Energy Return on Investment

ERI - Energy Return on Investment

EVP - Evaporator

FAME - Fatty Acid Methyl Esters (also known as biodiesel)

FLC - Flocculation tank

FRM - Fermenter

FU - Functional Unit

$\mathrm{GaBi}$ - German for "holistic balancing"

GGE - Gallon of Gasoline Equivalent

GHG - Greenhouse Gas

GLY - Glycerol

GPE - Green Process Engineering

GWP - Global Warming Potential

HRT - Hydraulic Retention Time 
HTL - Hydrothermal liquefaction

ICE - Internal Combustion Engine

ILCD - International Reference Life Cycle Data System

IO - Input /Output

ISO - International Organization for Standardization

LCA - Life Cycle Assessment

LCFS - Low Carbon Fuel Standard

LCI - Life Cycle Inventory

LCIA - Life Cycle Impact Assessment

LE - Lipid Extraction

LO - Left Over

LPG - Liquefied Petroleum Gas

$\mathrm{MeOH}$ - Methanol

MG - Monoglyceride fatty acid

MMLY - Million Metric Liters per Year

MTBE - Mthyl Tert-Butyl Ether

MXT - Mixing tank

$\mathrm{N}$ - Nitrogen containing compound

NA - Natural Gas

NER - Net Energy Ratio

NREL - National Renewable Energy Laboratory

ORP - Open Raceway Ponds

$\mathrm{P}$ - Phosphorus containing compound

PBR - Photobioreactor

PM - Particulate Matter

PUFA - Polyunsaturated Fatty Acid

RD - Renewable Diesel

RITE - Research Institute of Innovative Technology for the Earth

SAF - Suspended-Air-Floatation

SDTC - Sustainable Development Technology Canada

SHF - Separate enzymatic Hydrolysis and Fermentation

SRT - Solid Retention Time

SSF - Simultaneous Saccharification and Fermentation

STEM - Science, Technology, Engineering \& Mathematics

STX - Heat treating vesse19797

TG - Triglyceride Fatty Acid

TN - Total Nitrogen

TP - Total Phosphorus

TS - Total Solids

UR - Un-reacted

US - United States of America

WCI - Western Climate Imitative

WWT - Waste Water Treatment

VKT - Vehicle Kilometers Travelled 


\section{Chapter 1 - Introduction}

\subsection{Motivation for carrying out this study}

Studies have shown a direct link between anthropogenic GHG emissions and global warming (Bare et al., 2003; Bernstein et al., 2007; Obasi \& Tolba, 1992; Oreskes, 2004; Qin et al., 2013). This link was made back in the 1960s; however, the political climate at the time was not receptive to decarbonizing the economy (Oreskes, 2018). Presently, it is well-known that fossil resource use, including fossil fuels, is one of the leading causes of GHG emissions globally (Wittcoff et al., 2013). The extraction and use of fossil fuel adds additional carbon, in the form of GHG, to the earth's atmosphere that had previously been stored underground, thus exacerbating the global warming effect (Oreskes, 2004).

Given the continued use of liquid fuels for certain forms of transportation for the foreseeable future (Pond Technologies Inc., 2017; United States of America Department of Energy, 2015), one way to reduce transportation-related GHG emissions is to produce and use renewable fuels or biofuels. In short, biofuels sequester carbon dioxide, one of the GHGs, and release the same during use, thus recycling the same atmospheric carbon. If renewable energy is used to produce biofuels, then the use of biofuels can assist in preventing the increase of GHG in the atmosphere and an increase in GHG induced global warming (Jacobson, 2009).

By definition, a biofuel is any fuel derived from terrestrial plants, aquatic plants or animal matter (Knothe, 2010). Biofuels are classified as primary, first generation, second generation and third generation (Wu et al., 2014). As seen in Figure 1, classification of biofuels is by their feedstock. Primary biofuels are organic material burned directly to produce energy (Dragone et al., 2010). Wood and other unprocessed plant matter fall into this category. This material is impractical for transport fuel purposes for a couple reasons. First, burning wood, for example, in any transport vehicle today would be logistically unweildly. Second, burning unprocessed biomass has a reputation of creating air pollution due to incomplete combustion in areas of high population density where large amounts of energy is required (Sanhueza et al., 2009). 


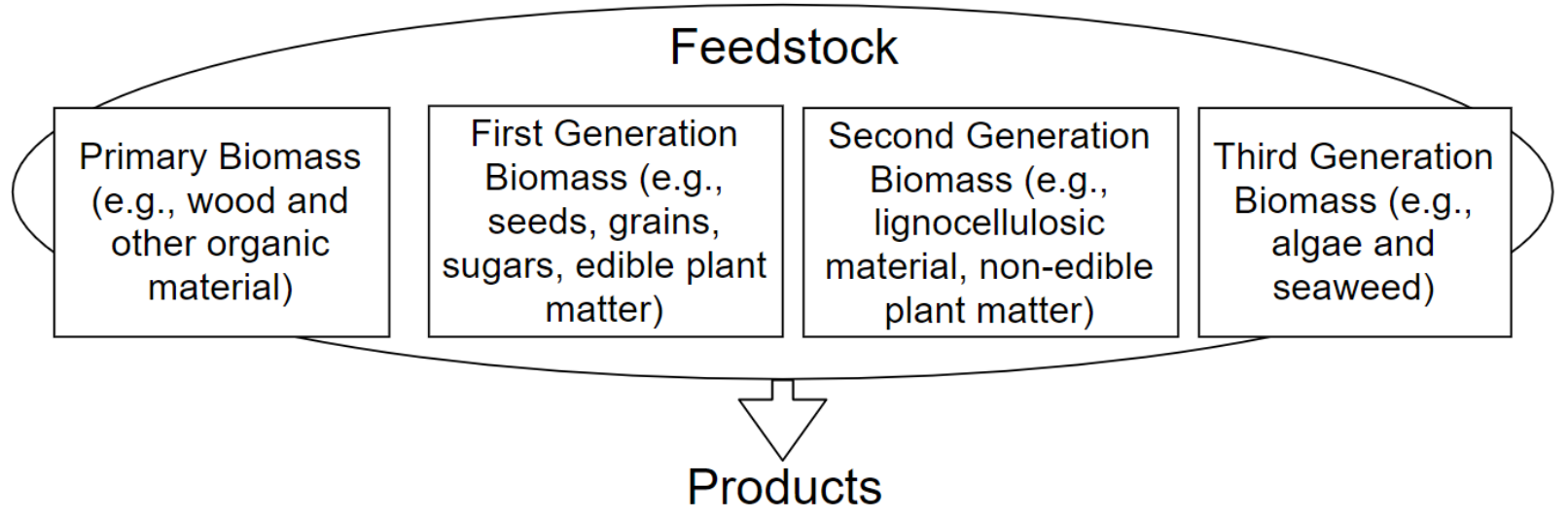

Figure 1.1: Types of biofuels, adapted from (Sharara et al., 2012)

First generation biofuels make up the majority of the biofuels used today. First generation biodiesel and ethanol biofuels produced today use vegetable oils (e.g., corn oil) and animal fats as their source feedstock (Dillon et al., 2008). There is a need to move away from relying on first generation biofuels because their feedstock would otherwise be human food (Suganya et al., 2016). With a growing population, it is more reasonable to use human food feedstock byproduct, known as second generation feedstock, to produce second generation biofuels (Suganya et al., 2016).

Second generation biofuel feedstock is the non-edible by-product of food crops. For example, wheat straw from wheat production and cornhusks from corn cultivation are second generation feedstock (Begum \& Dahman, 2015). There are advantages to using the inevitable by-product of the agricultural industry for biofuel production; no additional fertilizer, water or land is required to grow this feedstock. Industry does use some of this non-edible by-product to produce animal feed, however there is a substantial amount that could also be used for biofuel production (Syed, 2012). Expensive processes arguments against biofuel production from second generation feedstock plague this biofuel pathway (Sims et al., 2010). Regardless, second generation biofuel research and policy has the potential to develop this biofuel pathway into a productive source of biofuel (Balan, 2014; Begum \& Dahman, 2015; United States of America Department of Energy, 2017). 
Third generation biofuel production methods, like second generation biofuel production methods, are still more expensive than fossil fuel production methods. Consequently, the production of third generation biofuels, using algal feedstock, remains today predominantly at the pilot scale (Christenson \& Sims, 2011; Ribeiro et al., 2017). However, algae's ability to sequester carbon dioxide $\left(\mathrm{CO}_{2}\right)$, produce relative large amounts of lipids, grow in variable conditions, and grow orders of magnitude faster than all terrestrial plants (including second generation feedstock) make it an ideal biomass source for biofuels (Sharara et al., 2012; Wu et al., 2014). Several researchers have indicated that algae has a significant role to play in future liquid biofuel development (Chisti, 2007; Christenson \& Sims, 2011; United States of America Department of Energy, 2017). It is for the above reasons, this study focuses on biofuel derived from algae.

Microalgae and macroalgae are two ways of structurally classifying algae. Microalgae can be seen under a microscope and macroalgae is seen with the unaided eye (Radmer, 1996).

Microalgae is deemed more suitable for cultivation with the purpose of biofuel production because their small size takes up less cultivation area and most current algal research focuses on microalgae for the pharmaceutical industry (Suganya et al., 2016). With additional research, biofuel from macroalgae is possible. However, given the current state of research, this study's focus is on microalgal biofuel production.

Microalgae have been harvested for food from natural sources for hundreds of years in Mexico, Africa and Asia (Farrar, 1966). These microphytes were first cultured in a laboratory in the late part of the $19^{\text {th }}$ century and only once laboratory culturing was reliable did researchers start working on understanding microalgae's nutritional requirements and basic physiology (Soeder, 1986). It was in 1942 when Harder and von Witsch proposed microalgae's potential as a renewable fuel source (Harder \& von Witsch, 1942). The early 1950s saw commercial farming pilot project development (Burlew, 1953). The USA, Germany, Japan and Israel produced the first outdoor microalgal cultivation systems using the microalgal genus Chlorella (Burlew, 1953; Soeder, 1986). However, the need for liquid fuel alternatives dwindled after World War II and microalgal research focus turned to microalgae's potential as a food protein source (Geoghegan, 1951; Spoehr \& Milner, 1949). Microalgal nutrient development research in Asia continued, resulting in a present day successful Chlorella industry in Japan, Taiwan and throughout Asia 
(Borowitzka, 2013; Kawaguchi, 1980). The USA and Australia, in contrast, did not start producing different mass cultures in earnest for pharma and fish feed until the 1980s and 1990s (Belay, 1997; Borowitzka et al., 1984).

Microalgae was proposed as a wastewater treatment (WWT) option in 1957, which included the idea of using methane produced from the fermentation of microalgal biomass for energy generation (Oswald \& Gotaas, 1957). There has been little research concerning microalgal biomass fermentation since, however, research in the early 1960s performed by William Oswald at the University of California specifically focused on microalgal biomass production and wastewater treatment (Oswald, 1988). Oswald's research, as well as the 1970s energy crisis, lead to the critical assessment of the possibility of using microalgae for energy towards the end of the 1970s (Oswald \& Benemann, 1977).

The Aquatic Species Program (ASP) was initiated by the United States of America Department of Energy in 1978 (Sheehan et al., 1998). This study's conclusions played a critical role in the biofuel direction of North America. This study concluded that microalgal productivity (i.e., rapid growth) and conditions that lead to high oil content in microalgae were mutually exclusive. This means that rapid miroalgal growth would be synonymous with a reduced lipid (i.e., oil) content, thus discouraging microalgal potential as a feedstock for BD production. The ideal organism(s) for biofuel production would likely be location specific (Sheehan et al., 1998), hence requiring an understanding and practice of traditional knowledge (McGregor, 2004).

Interestingly, the ASP study specifically indicated that there was "no fundamental engineering and economic issues that would limit the technical feasibility of microalgae culture (from open pond) either in terms of net energy inputs, nutrient (e.g., $\mathrm{CO}_{2}$ ) utilization, water requirements, harvesting technologies or general system designs" (Borowitzka, 2013). However, the study recommended that microalgal biofuel development transpire in consort with WWT where economic and resource constraints are relaxed; wastewater would provide required nutrients for microalgal growth, and microalgal productivities could be developed. Consequently, the development of microalgal biofuel in North America stagnated.

The Japanese Ministry of International Trade and Industry initiated the RITE Biological $\mathrm{CO}_{2}$ Fixation Programme in 1990 (Borowitzka, 2013). Although the program was focused on sequestering $\mathrm{CO}_{2}$ and not specifically microalgal energy production, some of this research 
improved knowledge of photobioreactor design using solar capture and radiance. This research also provided a better understanding of high $\mathrm{CO}_{2}$ tolerant microalgal strains that could thrive on flue gas (Usui \& Ikenouchi, 1997).

Now in 2018, a Government of Canada Ministry of Environment and Climate Change objective is to prevent additional global warming by sequestering $\mathrm{CO}_{2}$ and reducing $\mathrm{GHG}$ emissions. The Canadian Foundation for Sustainable Development Technology Federal Act of 2001 and the Federal Sustainable Development Act of 2008 provide a management and funding framework for sustainable technological development in Canada in order to support this government objective (The Government of Canada, 2001, 2008). The Canadian government renewable fuel strategy and associated mandates of $5 \%$ and $2 \%$ renewable fuel (i.e., biofuel) in gasoline and diesel respectively, also supports this government objective (Government of Canada, 2017). Additional supporting provincial objectives and policies, such as the CleanTech strategy in 2018, have created a favourable environment for developing microalgal biofuel (The Government of Ontario, 2018). Given the current political climate, now (2018) is a good time to focus on developing the aforementioned integrated WWT and microalgal biofuel production system proposed by the ASP program (Ferrell \& Reed, 2010).

As seen in Figure 1.2, microalgae can be used to produce several different types of biofuels by various pathways (Wu et al., 2014). Processes a) through f) use various components of the microalgal biomass to produce the biofuels on the far right of Figure 1.2.

Thermochemical processes, as seen in Figure 1.2 d) through f), do not use each component (i.e., lignocellulosic, lipid, protein) of the microalgal biomass separately to produce different products. Rather, microalgal biomass is directly converted to one product resulting in heating or power generation as the only use for the left over waste (Broch et al., 2014; Frank et al., 2013). For example, Frank et al. (2013) produced renewable diesel (RD) using hydrothermal liquefaction (HTL) and subsequently routed all non-oil biomass through a catalytic hydrothermal gasification $(\mathrm{CHG})$ processor to produce biogas for heat generation, power generation and additional aqueous waste. The likely advantage of thermochemical processes is the reduced processing up-front with the resulting disadvantage of substantial follow-on processing in order to produce a variety of products (Broch et al., 2014; Frank et al., 2013). GHG impact for thermochemical processes 
could be more or less intense pending waste or co-product handling and processing methods (Frank et al., 2013).

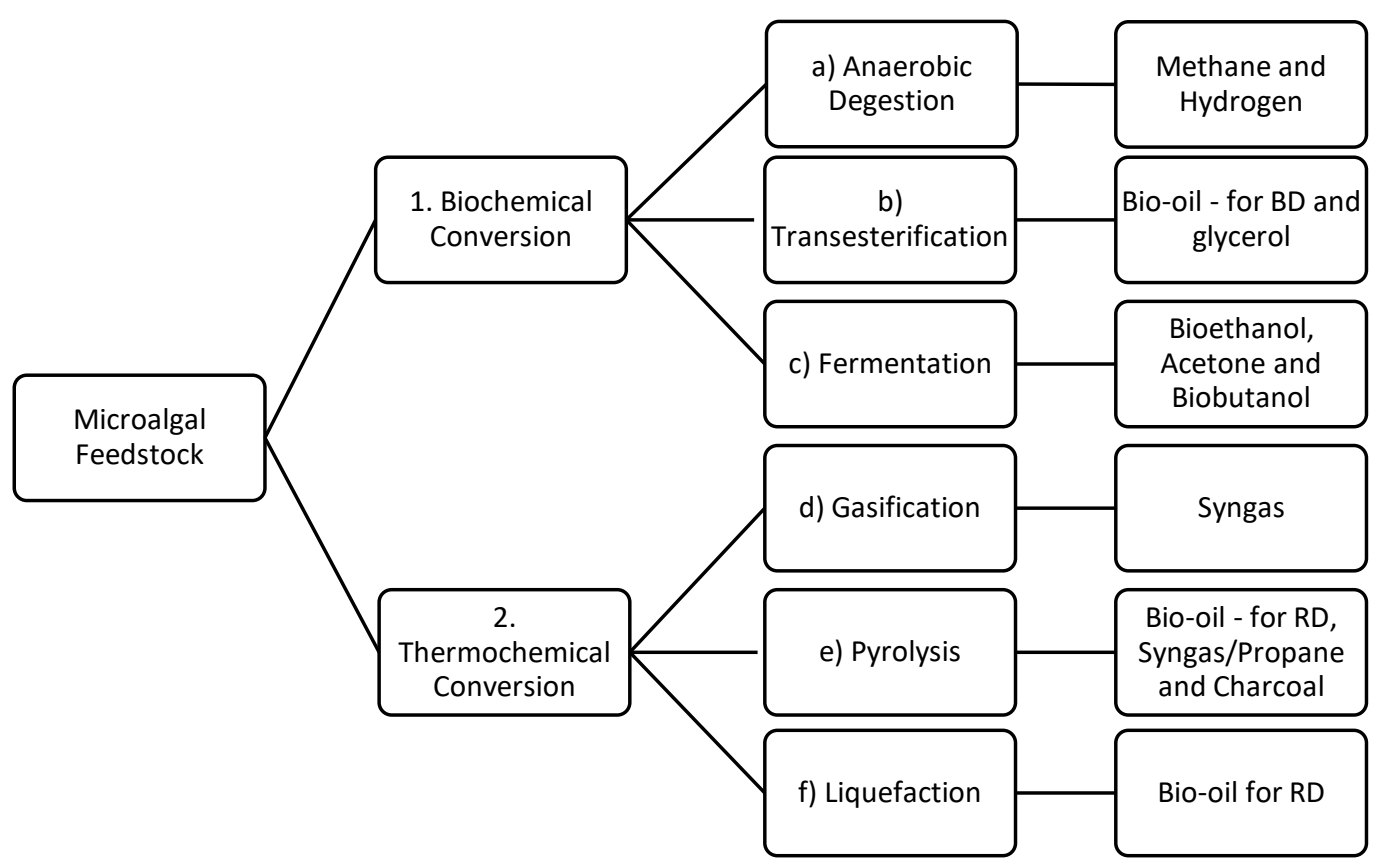

Figure 1.2: Biofuel production process pathways form microalgal biomass, BD - biodiesel, RD renewable diesel, adapted from (Brennan \& Owende 2010; Suganya et al. 2016; Wu et al. 2014)

In contrast, biochemical pathways b) and c) use selective components of the microalgal biomass to produce respective products (Dong et al., 2016). For example, fermentation targets lignocellulosic biomass to produce alcohols and transesterification targets lipids to produce fatty acid methyl esters (FAME). Biochemical processes have the opposite advantages and disadvantages of thermochemical processes mentioned above. Furthermore, GHG impact for biochemical processes could be more or less the same as that of thermochemical processes for the same reasons. Therefore, the only way to predict GHG impact is to create a process flow analysis of the process in question and determine emissions for each material required.

A key concept that influenced this study's selection of unique microalgal biofuel production processes is the Biorefinery concept depicted in Figure 1.3. 


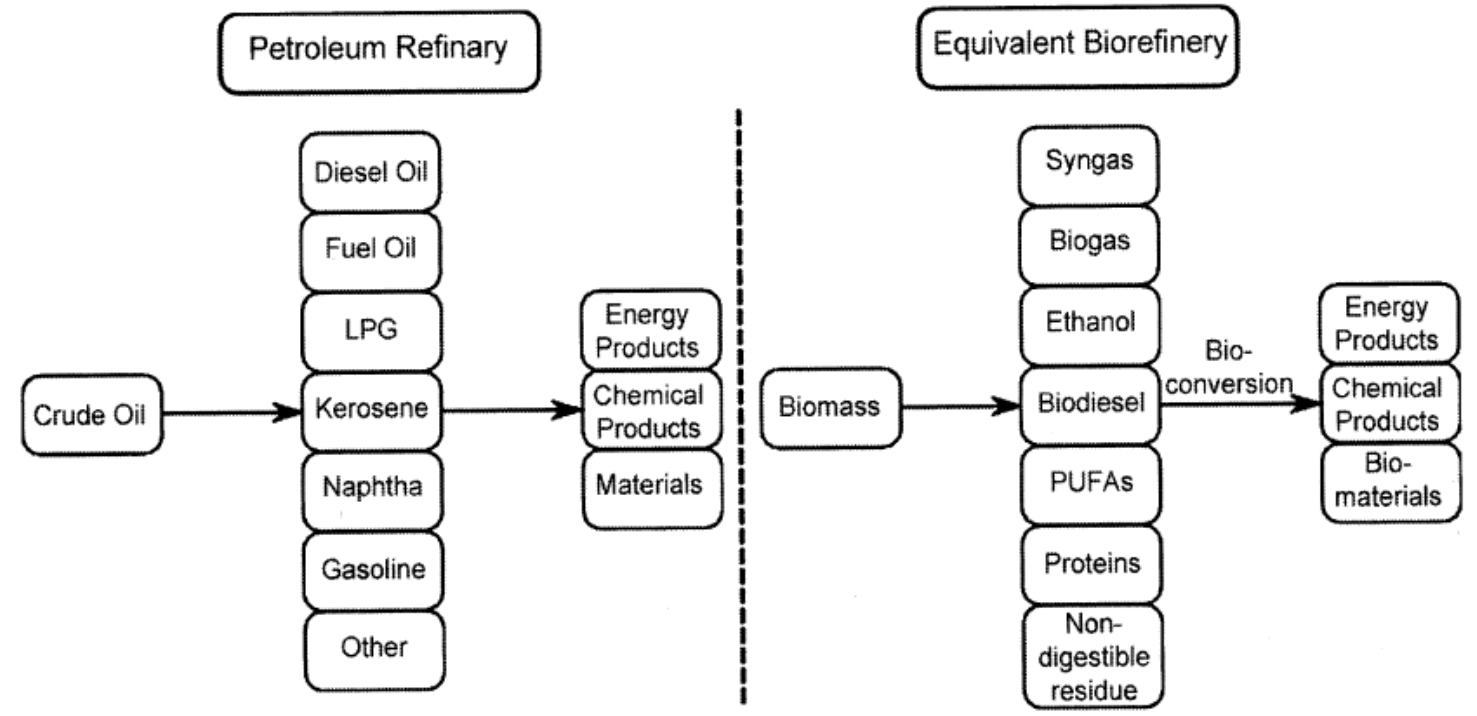

Figure 1.3: Analogy of a bio-refinery approach for algal biomass with a petroleum refinery, LPG - liquefied petroleum gas, PUFA - polyunsaturated fatty acids (Sharara et al., 2012)

A bio-refinery is very similar to the petroleum refineries in operation today except with two key differences. First, petroleum refineries use crude oil, a fossil resource, whereas a bio-refinery would use biomass. Second, petroleum refineries use one feedstock, crude oil, and produce a multitude to intermediate products (e.g., diesel oil, LPG, naphtha) that are then used to produce most of the plastics, fuels and chemicals used today (Wittcoff et al., 2013). In contrast, a biorefinery is flexible enough to use several biomass feedstock (e.g., corn, wheat straw, algae etc.) and produce bio-plastics, bio-fuels and bio-chemicals (Sharara et al., 2012). Not only would a bio-refinery be able to produce products from several biomass feedstocks, it would also have the ability to modify its production line at any time to produce more or less of a given product pending feedstock availability. It is anticipated that because biomass feedstock is either terrestrial or aquatic, an increase in extreme weather (e.g., unexpected drought or flooding) would make certain feedstock availability more of less reliable (United States of America Department of Energy, 2015).

It is not inacurate to describe the fuel and chemical industry of the early $20^{\text {th }}$ century as users of the Biorefinery concept. Most industrial materials at the time (e.g., dyes, solvents, synthetic fibers) were produced using trees and agricultural crop (Ragauskas et al., 2005). However, the 
need for recycling would not have been as critical as it is now. The Biorefinery concept, for the $21^{\text {st }}$ century's purpose, was coined two decades ago (Romero-García et al., 2014; Zhu, 2015). Current research and government reports use this phrase to define an old concept in light of the need to revert to bio-based materials (i.e., materials derived from terrestrial plant or aquatic matter instead of fossil resources) (Petrick et al., 2013; Romero-García et al., 2014; The Government of Ontario, 2018).

Both thermochemical and biochemical processes are suitable bio-refinery processes. Both groups of processes also have the potential to source multiple feedstocks and produce multiple products (Frank et al., 2013). However, biochemical processes b) and c) of Figure 1.2 target the different components (i.e., lipid, lignocellulosic biomass, protein) of biomass that are common to all biomass (Huber \& Corma, 2007). This targeting allows for the production of BD, biogas, biosolids as well as alcohol biofuels with few processing steps. These bio-based alcohols can also be used as feedstock for bio-chemicals (Zhu, 2015). Therefore, analyzing the production of both microalgal BD and alcohol using a Biorefinery concept and fervid recycling will shed light on the potential development of production processes that use biological processes to produce a variety of fuels and bio-based materials.

Because of microalgae's potential for high lipid content, and lipids are the main component of BD, progress in microalgal biofuel research focuses on BD (USA D.O.E., 2017; Yoo et al., 2009). Therefore, this study's main product is BD. However, considering that the lipid content in $\mathrm{BD}$ is variable, it is advantageous to capitalize on the readily available lignocellulosic component of the microalgal biomass to produce concurrent products (United States of America Department of Energy, 2017). Pre-treatment can breakdown microalgal lignocellulosic material to simple sugars for subsequent alcohol biofuel production by fermentation (Chen et al., 2013). There is potential for alcohol biofuel to play a larger role in transportation in the future (Tashiro et al., 2013) and there are few studies that focus on microalgal lignocellulosic biomass processing for biofuel (Chen et al., 2013). Consequently, there is no literature available that characterise the GHG impact of the production of both microalgal BD and ABE. Therefore, this study will analyze the GHG impact of the production of BD and ABE.

This biofuel GHG process analysis, similar to other environmentally focused biofuel process analyses, uses life cycle analysis (LCA) methodology (Spirinckx \& Ceuterick, 1996). LCA 
methodology includes methods to assist with compiling all stages of the life cycle of a product process system to analyze select environmental impacts of the product (International Organization for Standardization (ISO), 2006a; Sinden et al., 2010). For example, scope development, co-product allocating, impact category selection, and aggregating software tools are tailorable aspects of LCA methodology used to analyze the environmental impact of a product pending study objectives.

\subsection{Objective}

The purpose of this study is to determine the GHG impact associated with a unique production process of microalgal $\mathrm{BD}$ and $\mathrm{ABE}$. The results of this study will provide a reference to contrast the resulting GHG impact with that of other microalgal BD production processes and the fossil diesel production process.

\subsection{This study's intended application and audience}

This study follows an attributional LCA approach to quantify the GHG impacts associated with a production process. An attributional LCA approach describes relevant environmental physical flows (e.g., water, material, energy) to and from the processes life cycle system (Finnveden et al., 2009). The results of an attributional LCA use these physical flows to determine the environmental impact (e.g., GHG emissions, land use, eutrophication impact) of the process. Most LCA studies fall into this attributional category, as it is relatively methodical to determine the predominant physical flows of an existing or theoretical system. In contrast, the consequential LCA approach describes how environmentally relevant ecosystem flows will change in response to the introduction of a process (Finnveden et al., 2009). This LCA approach is much more difficult than the attributional approach because the consequential approach requires the quantification of how relevant process flows (e.g., GHG emissions, water use) will influence these same environmentally significant ecosystem components (e.g., GHG levels, water flow).

Given the complexity and expertise required to complete a full attributional LCA, this study will not contain all the recommended parts associated with an attributional LCA as defined by 
Collotta et al. 2016 (i.e. full range of impact categories, social and economic indicators). This study will only contain the Climate Change impact category and associated Global Warming Potential (GWP) categorization factor measured in terms of GHG emissions. Regardless, this study fulfils the requirements a) through i) of paragraph 5.3.1 of ISO 14044:2006 such that this study will meet the requirements for disclosure to the public (International Organization for Standardization, 2006b).

This study's intended audience is not necessarily decision makers who are looking for a complete environmental impact assessment. Instead, those who would benefit from this thesis are decision makers looking for opportunities for an industry-government partnership focused on facility system integration and green process engineering systems. This paper would also provide a good breakdown of the LCA approach, including a good description of the use of software tools and co-product allocation reasoning. The LCA description would be useful for managers in industry who are looking to develop their environmental management system (EMS). Finally, this paper would provide interested educators with a brief history of fuel use, the basic principles of microalgal biofuel production and a good introduction to LCA.

In this study, the term "microalgae" is used when discussing the organism in singular as well as plural. The plural term for microalgae is microalga. The reason for this choice is first, the paper's intended audience are experts in policy, engineering or business and not botany. Second, most of the papers this study references on the topic of microalgae use the term "microalgae" ubiquitously.

It is important to mention here that due to the limits of GaBi Education, this study relies predominantly on GHG emission data from American (i.e., United States of America) sources. All electrical power energy and natural gas GHG impact use average American aggregated impact data. This created the least geographic variability in source data while at the same time used North American data instead of European data. However, as GaBi Education is of German origins, this study used European data when American data was not available. Given the aforementioned asymmetries, the reader should also understand that more fossil energy supplies the American power grid than the Canadian power grid. Due to Canada's hydroelectric 
resources, Canada's fossil fuel use for power generation is approximately one third that of the United States (Johnson et al., 2016; Natural Resources Canada, 2015b; Oil Sands Magazine, 2017; U.S. Energy Information Administration, 2017). Chapter 4 and 5 discuss how this power source difference affects the results of this study.

Given this study found a direct correlation between GHG impact and power use, there would be little value in completing a GHG impact assessment of a new energy production process if the prospective implementation location has a fossil intensive power generation system. Future research in the area of microalgal biomass energy production processes should focus first on ensureing that the process is as energy efficient as possible, and then look at sustainable sources of power and energy to supply that which is required. In the future, researchers and LCA practitioners should see the GHG impact of all processes decrease as power and energy generation move to sustainable processes. Considering energy and power are such key commodities for a modern society, new energy production systems are likely to become widespread. It is imperative because of the broad impact of these systems that researchers ensure that new energy production systems have life cycles that do not significantly affect other areas of the environment. Future LCAs on aforementioned energy production systems can be tailored to help assess other key environmental impacts such as eutrophication, land use impact, species impact, water consumption etc.

This study is written using a socially adpated technical style of writing. The author's intention is to ensure those with a less technical background will easily understand its contents as well as the meaning behind the contents. Considering environmental science issues are interdisciplinary, and often decision makers have varied levels and areas of scientific expertise, there is value in creating literature that bridges the gap between social science and STEM (Choi et al., 2005; Guston, 2001). Therefore, most should be able to understand the majority of the points herein.

\subsection{Document outline}

Chapter 2 of this document contains the review of literature covering:

- A brief history of biodiesel and ABE use in North America

- Current biofuel policy and Canadian government goals concerning biofuel 
- Microalgae's potential as a feedstock for biofuel

- Microalgal BD and ABE processing methods

- Current life cycle assessments of microalgal BD and co-products

Chapter 3 of this document outlines the research process. This includes a detailed description of the LCA method used to quantify and assess the GHG impact associated with the unique microalgal $\mathrm{BD}$ and $\mathrm{ABE}$ production process.

Chapter 4 presents GHG impact associated with the study's unique microalgal BD and ABE production process in relation to other LCAs of microalgal BD outlined in Chapter 2. This chapter also discusses consistancies, uncertainty and discrepancies between this study and others. Chapter 5 presents potential areas of improvement, conclusions and recommendations. 


\section{Chapter 2 - Biofuel production history and modern microalgal biofuel production advancements}

\subsection{Production and use of biofuel and their fossil counterparts}

Note that in the following section 2.1, "bio" in front of a product name distinguishes products derived from biomass with their fossil counterpart. For example, bio-ethanol is derived from biomass, whereas ethanol is a product of fossil resources. Where brackets are used around the word bio (i.e., (bio)), both the bio-product AND fossil product should be considered in context. For example, both ethanol and bio-ethanol are chemically interchangeable, therefore when referring to the product chemical characteristics, (bio)ethanol is written to imply both bio-ethanol or ethanol.

The following section presents the primary materials used to produce (bio)diesel, (bio)ethanol, (bio)butanol and (bio)acetone as well as how (bio)diesel, (bio)ethanol, (bio)butanol and (bio)acetone are used. The section also includes the advantages and disadvantages of using the fuels bio-diesel, bio-ethanol and bio-butanol for energy in a combustion engine as neat fuels or in blends. Neat is a term used for $100 \%$ or without blending.

\subsubsection{Biodiesel}

Petroleum, natural gas and coal are the three large groups of fossil fuels. Crude oil is part of the myriad of material derived from petroleum and diesel is a fossil fuel derived from crude oil processing (Knothe, 2010). In contrast, BD is derived from a plant oil or animal fat (Knothe, 2010).

Both $\mathrm{BD}$ and fossil diesel fuel compression ignition engines (CIE). BD and fossil diesel are made up of fatty acid esters and $n$-alkane chains respectively (Wu et al., 2014). Figure 2.1 depicts a typical fatty acid ester. The fatty acid ester has a carboxylic functional group (circled in Figure 2.1), whereas fossil diesel is a simple alkane or a hydrocarbon chain (see Figure 2.1). The hydrocarbon chain of both the fatty acid esters and $n$-alkane hydrocarbons used for fuel 
production most often contain between 16 and 22 carbon atoms connected together with either single or double bonds (Knothe, 2010).

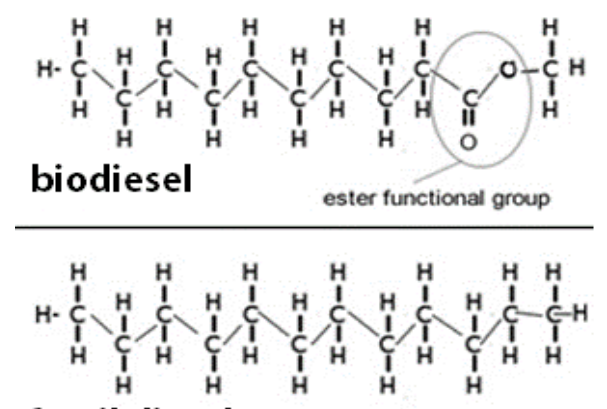

fossil diesel

Figure 2.1: Biodiesel and fossil diesel basic chemical formula, adapted from (Wu et al., 2014)

$\mathrm{CN}$ is a metric used to measure the ignition quality of diesel fuel and is an important characteristic of both fossil diesel and biodiesel. The higher the $\mathrm{CN}$ number, the less delay between fuel compression and ignition, which generally results in more complete combustion and a more efficient engine. The more unsaturated (i.e., more double bonds) the fuel's hydrocarbon chains, the lower the cetane number $(\mathrm{CN})$ of the fuel, the lower the viscosity of the fuel, the higher the biodegradation potential of the fuel, and the higher the lubricity of the fuel (Suganya et al., 2016; Zaimes \& Khanna, 2013). The opposite is true for fuel hydrocarbon chains that are more saturated (i.e., more hydrogen atoms) (Suganya et al., 2016). Longer hydrocarbon chains increase the $\mathrm{CN}$ of the fuel, increase the viscosity of the fuel, and increase the lubricity of the fuel (Suganya et al., 2016). The opposite is generally true for shorter hydrocarbon chains (Suganya et al., 2016). Hydrocarbons with branching and aromatic compounds tend to lower the fuel's CN and decrease the viscosity of the fuel (Knothe, 2010).

BD tends to have a lower CN, a slightly lower HV (heating value), a slightly higher viscosity, a higher biodegradability, an increased NOx emission level (i.e., +10\%), consistent or higher particulate matter (PM) emissions, lower hydrocarbon (i.e., unburnt fuel) emissions and lower carbon monoxide (CO) emission level when compared to fossil diesel (Corriere et al., 2013; Igbokwe \& Nwafor, 2014; Knothe, 2010; Mattarelli et al., 2015). A lower CN and a lower HV have the greatest potential to dissuade BD use as they pose operational interference to the vehicle end user. For example, a lower $\mathrm{CN}$ has the potential to affect engine start timing and reduce 
engine efficiency. A lower HV increases the amount of fuel required per kilometer. Regardless, microalgal biodiesel's physical and chemical properties are quite similar to that of fossil diesel (see Table 2.1) and are appropriate based on ASTM fuel standards found in Table 2.1.

Therefore, microalgal BD will provide sufficiently comparable engine performance to petrol diesel (Maio \& Wu 2006). Consequently, GHG emission impact, associated with burning both $\mathrm{BD}$ and petrol diesel in a CIE, are virtually the same.

Table 2.1: Properties of algal biodiesel, fossil diesel and ASTM biodiesel standards ((a) = Brennan \& Owende, 2010; (b) = Maio \& Wu, 2006; (c) = ASTM INTERNATIONAL, 2014)

\begin{tabular}{|l|l|l|l|}
\hline Properties & $\begin{array}{l}\text { Biodiesel from } \\
\text { microalgal oil (a) }\end{array}$ & Diesel Fuel (b) & $\begin{array}{l}\text { ASTM biodiesel } \\
\text { standard (c) }\end{array}$ \\
\hline Cetane $(\mathrm{CN})$ & $45-60$ & $90-100$ & Minimum 47 \\
\hline Density $(\mathrm{kg} / \mathrm{L})$ & 0.864 & 0.838 & $0.86-0.9$ \\
\hline $\begin{array}{l}\text { Viscosity }\left(\mathrm{mm}^{2} / \mathrm{s} \mathrm{cSt}\right. \\
\left.\text { at } 40^{\circ} \mathrm{C}\right)\end{array}$ & 5.2 & $1.9-4.1$ & $3.5-5$ \\
\hline Flash point $\left({ }^{\circ} \mathrm{C}\right)$ & 115 & 75 & Minimum 100 \\
\hline $\begin{array}{l}\text { Cold filter plugging } \\
\text { point }\left({ }^{\circ} \mathrm{C}\right)\end{array}$ & -11 & -3 to max -6.7 & $\begin{array}{l}\text { Summer max } 0, \\
\text { winter max }<-15\end{array}$ \\
\hline $\begin{array}{l}\text { Acid value }(\mathrm{mg} \\
\text { KOH/g) }\end{array}$ & 0.374 & Max 0.5 & Max 0.5 \\
\hline $\begin{array}{l}\text { Lower heating value } \\
(\mathrm{MJ} / \mathrm{kg})\end{array}$ & 41 & $40-45$ & No requirement \\
\hline $\mathrm{H} / \mathrm{C} \mathrm{ratio}$ & 1.81 & 1.81 & No requirement \\
\hline NOx, PM, HC, CO & - & - & No requirement \\
\hline
\end{tabular}

Based on the characteristics in Table 2.1, BD (B100) also known as neat BD, is compatible for use in light vehicle diesel engines without blending or reduced engine life expectancy (Al-Hasan, 2013; Knothe, 2010). The US military has run vehicles and ships on pure biodiesel (Kumar \& Jain, 2014; Pond Technologies Inc., 2017). Yet, although there are currently 10 operational BD plants in Canada producing over 662.2 million metric liters of fuel per year (mmly) (Biodiesel Magazine, 2017), there is no mainstream neat BD or even RD available at pumps in Canada. The capacity of BD operating plants in Canada range from 0.2 mmly (Cowichan BD cooperative uses recycled waste cooking oil) to 265 mmly (Archer Daniels Midland Co. uses vegetable oil) (Archer Daniels Midland Co., 2018; Cowichan Biodiesel Co-op, 2015). Local business owners run some of the smaller plants and supply local populations, thus those living in 
the area are the only people who benefit. Furthermore, given Canadians consumed 13.8 billion liters of diesel fuel in 2015 (Statistics Canada, 2015), most renewable BD produced is either blended to support the Canadian Federal Government's renewable fuel component mandate or is sold to the United States to support their mandates.

\section{Renewable Diesel (RD)}

$\mathrm{BD}$ and RD are not synonymous. The "bio" in biodiesel also refers to the biodegradability of biodiesel because of the limited refining used for production (Knothe, 2010). The transesterification process produces BD from FAMEs and FAMEs are a component of terrestrial and aquatic plant oils. Similarly, terrestrial and aquatic plants are the bio-feedstock of RD. In contrast to $\mathrm{BD}$, however, extensive processing modifies the bio-feedstock such that the final RD product has the chemical and ultimately the combustion characteristics of fossil diesel (Knothe, 2010). RD is also often called "Green diesel". The "green" applies to the origin of the feedstock (i.e., bio-feedstock), but does not refer to the fuel's biodegradability, which is nullified to an extent by the subsequent modifications performed to emulate fossil diesel (Knothe, 2010).

Introduced in section 1.1 and depicted in Figure 1.2, microalgal biomass is subject to thermochemical processes to produce RD (Suganya et al., 2016). In fact, both microalgae and BD can be used as a feedstock to produce RD with thermochemical processes (Knothe, 2010; Wiens et al., 2011). Today, to produce RD from microalgae, microalgae would be subject to pyrolysis or hydrothermal liquefaction (Frank et al., 2013). To produce RD from BD, BD would be subject to hydrocracking. Hydrotreating, including hydrocracking, is an established refinery process used to reduce sulfur, nitrogen and aromatics from a fuel while enhancing the cetane number, density and smoke point (Shell Global, 2017). Catalysts such as $\mathrm{NiMo} / \gamma-\mathrm{Al}_{2} \mathrm{O}_{3}$ or $\mathrm{CoMo} / \gamma-\mathrm{Al}_{2} \mathrm{O}_{3}$, at high temperatures and pressures, are used to produce saturated alkanes by removing the carboxyl group from the alkane (Frank et al., 2013; Knothe, 2010).

Industry publically favours RD over BD (Honeywell UOP, 2018; Neste, 2018). Also in favour of RD, Kalnes et al. (2009), argue that RD production requires less fossil-based energy than BD (Kalnes et al., 2009). However, Frank et al. (2013) find little difference in life cycle GHG impact between their RD and BD processes (Frank et al., 2013). Regardless, both BD and RD 
can be stored, transported and used the same way, therefore the preference for RD is likely because RD is chemically indistinguishable from fossil diesel, whereas BD is not. RD is a drop in fuel, whereas the use of BD likely requires small, but significant industry accommodations (e.g., variation in fuel LHV, different fuel additives, changes to material safety data, additional human resources training etc.). Furthermore, RD, a more refined fuel, prevents certain types of air pollution (i.e., NOx and PM), which follows the same historical reasoning used to cease primary biofuel use in urban areas outlined in section 1.1 (Kalnes et al., 2009). Regardless, there is likely a place for both RD and BD instead of opting for one or the other.

\subsubsection{Ethanol}

Ethanol is produced by the hydrolysis of ethylene, a petrochemical, whereas bio-ethanol is produced by the fermentation of several different types of feedstock: corn, soybean, sugarcane, wheat straw, woodchips or algae (Arbor, 1986; Hira \& De Oliveira, 2009; Mills \& Ecklund, 1987; Wittcoff et al., 2004). Producing bio-ethanol by fermentation for vehicles was quite common until the advent of cheap ethylene from steam cracking in the 1950s (Wittcoff et al., 2004). Once federal tax credits favoured bio-ethanol production in the 1980s after the energy crisis, the fermentation process became more widely used again in the United States (Mills and Ecklund 1987; Wittcoff et al., 2004). In Canada, federal and provincial incentives in the first part of the $21^{\text {st }}$ century contributed to the bio-ethanol industry's growth (Le Roy \& Klein, 2012)

Both ethanol and bio-ethanol are chemically indistinguishable (same properties) as they are both the same compound. This makes both synthetic and bio versions interchangeable in end-uses. Figure 2.2 depicts the chemical formula of (bio)ethanol. Presently, ethanol is used as a blend in gasoline, as a neat fuel for burners and as a solvent in the chemical and pharmaceutical industry (Hira \& De Oliveira 2009; Linden et al., 1985; Mills and Ecklund 1987). Whereas, bio-ethanol is primarily used as a blend in gasoline, given government regulations mandating a certain percentage of biofuel blended with fossil fuel. 


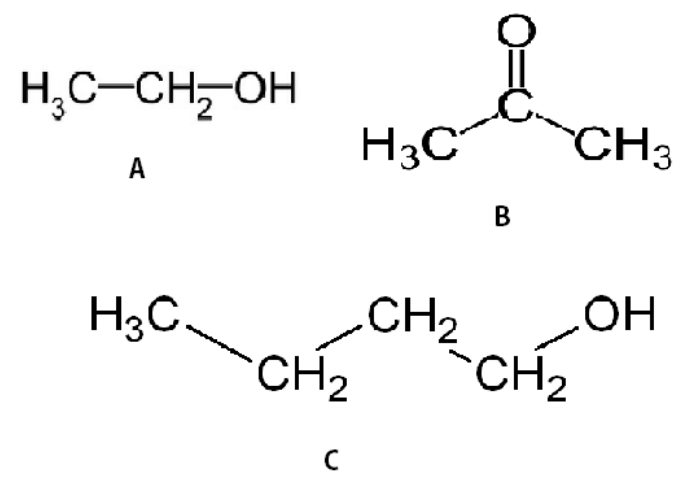

Figure 2.2: Chemical formulas of (bio)acetone (B), (bio)ethanol (A) and (bio)butanol (C), Note (bio)butanol or n-butanol has 3 other isomeric structures: isobutanol, 2-butanol and tert-butanol not shown here, adapted from (Monick, 1968)

There are several advantages to using (bio)ethanol in fossil fuel blends. One such advantage is the increase in the fuel's ability to achieve complete combustion due to the reduction of unburnt hydrocarbons, as (bio)ethanol contains a hydroxyl group (i.e., naturally oxygenated) (Yüksel \& Yüksel, 2004). (Bio)ethanol blends also have higher octane ratings due to a high engine compression ratio (fuel can withstand a higher pressure before igniting) resulting in improved engine timing and increased efficiency (Yüksel \& Yüksel, 2004). Environmentally, (bio)ethanol blends have been found to lower $\mathrm{CO}$ and NOx emissions upon combustion without large engine modifications (Yüksel \& Yüksel, 2004). (Bio)ethanol blends of 5-10\% are quite common in today's gasoline blends and require no engine modifications (Smerkowska, 2011).

(Bio)ethanol's auto ignition temperature and flash point are higher than gasoline, which makes it safer for transportation and storage (Yüksel \& Yüksel, 2004). Finally, (bio)ethanol also has a low freezing point, which makes it a good freezing inhibitor in fuel systems.

When used as a fuel in combustion engines, neat (bio)ethanol is more difficult to vaporize at cold temperatures, as (bio)ethanol has a higher auto ignition temperature and lower vapour pressure than gasoline (see Table 2.2) (Hansen et al., 2004). (Bio)ethanol has also been found to cause some degradation of engine components as (bio)ethanol and water are miscible and therefore have the potential to separate the alcohol and gas blend resulting in more frequent contamination and corrosion (Arbor, 1986; Hira \& Guilherme De Oliveira, 2009; Yüksel \& Yüksel, 2004). Another disadvantage of using neat ethanol from an end user perspective is that a vehicle will 
require more frequent fill-ups than if the vehicle ran on gasoline. This is because more (bio)ethanol is required per kilometer of car mileage; 1.5-1.8 times the alcohol fuel to achieve the same energy output (see LHV in Table 2.2) (Hira \& De Oliveira, 2009; Yüksel \& Yüksel, 2004). Therefore, (bio)ethanol is usually blended with gasoline to reduce its negative effects while capitalizing on the positive aspects of (bio)ethanol fuel (Yüksel \& Yüksel, 2004).

Regardless of the drawbacks of neat (bio)ethanol fuel outlined above, combustion engines can and have been modified to suit (bio)ethanol use. Against popular belief, cars that run on neat (bio)ethanol can also operate reliably in the Canadian climate (Arbor, 1986). The engine timing is modified as the fuel/air ratio required for (bio)ethanol combustion is different from that of gasoline combustion (Yüksel \& Yüksel, 2004). Engine component materials are modified to reduce the risk of corrosion (Hira \& De Oliveira, 2009). There are also flexible fuel engines that run on neat alcohol, neat gasoline or any combination of the two as they have computerized system that analyzes fuel properties and adjusts engine parameters accordingly (Arbor, 1986). These flexible engine vehicles were prototyped back in the 1980s and are only now starting to appear on roads in Ontario.

Table 1.2: Properties of (bio)ethanol, (bio)butanol and gasoline (Moo-Young, 1985*; Rakopoulos et al., 2011***; Schädlich et al., 2003**; Szwaja \& Naber, 2009*****; Yüksel \& Yüksel, 2004)

\begin{tabular}{|l|l|l|l|}
\hline Characteristics & $($ Bio)ethanol & $($ Bio $)$ butanol & Gasoline \\
\hline Formula & $\mathrm{C}_{2} \mathrm{H}_{5} \mathrm{OH}$ & $\mathrm{C}_{4} \mathrm{H}_{9} \mathrm{OH}^{* *}$ & $\mathrm{C}_{4}$ to $\mathrm{C}_{12}$ \\
\hline $\begin{array}{l}\text { Density }(\mathrm{kg} / \mathrm{L} \text { at } \\
\left.15^{\circ} \mathrm{C}\right)\end{array}$ & 0.79 & 0.81 & $0.69-0.79$ \\
\hline $\begin{array}{l}\text { Vapour pressure }(\mathrm{kPa} \\
\left.\text { at } 38^{\circ} \mathrm{C}\right)\end{array}$ & 15.9 & $2.1^{*}$ & $48-103$ \\
\hline Flash point $\left({ }^{\circ} \mathrm{C}\right)$ & 13 & 35 & -43 \\
\hline $\begin{array}{l}\text { Auto ignition temp } \\
\left({ }^{\circ} \mathrm{C}\right)\end{array}$ & 423 & 343 & 257 \\
\hline $\begin{array}{l}\text { Viscosity }(\mathrm{mPa} / \mathrm{s} \text { at } \\
\left.20^{\circ} \mathrm{C}, \text { at } 25^{\circ} \mathrm{C} * * * *\right)\end{array}$ & 1.19 & $2.57 * * * *$ & $0.37-0.44$ \\
\hline $\begin{array}{l}\text { Lower heating value } \\
(\mathrm{MJ} / \mathrm{kg})\end{array}$ & $26.8 * * *$ & $\begin{array}{l}36,681.0 \mathrm{~kJ} / \mathrm{kg}^{*} \\
33.1^{* * *}\end{array}$ & $30-33$ \\
\hline Octane number & 89.7 & $86 * * * *$ & $80-90$ \\
\hline
\end{tabular}




\subsubsection{Butanol}

Butanol is produced from propylene by hydroformylation (Wittcoff et al., 2004) and bio-butanol is produced by the fermentation of a variety of feedstock such as wheat, rye, wood, molasses, cheese whey etc. (Linden et al., 1985). In 1912, Weizmann discovered Clostridium acetobutylicum fermented starchy grains and produced bio-acetone, bio-butanol and bio-ethanol (Linden et al., 1985). At this time, acetone was the important product as it was used to produce cordite for naval ammunition (Linden et al., 1985). Therefore, Canadian plants used the Weizmann process to produce ABE to support the WWI war effort in Europe and continued using this method to support bio-butanol production for DuPont's post war production of nitrocellulose lacquers for the automobile industry (Linden et al., 1985). Like bio-ethanol production, with the advent of cheap petrochemicals in the 1950s, the fermentation of biomass to produce bio-butanol disappeared (Linden et al., 1985).

Similar to ethanol and bio-ethanol, butanol and bio-butanol are chemically interchangeable and can be used in the same end-uses. Figure 2.2 depicts the chemical formula of (bio)butanol. Butanol is used as a solvent and as a chemical intermediate; it is used as a solvent for waxes, resins, shellacs and varnishes as well as an intermediate to manufacture lacquers, rayon, detergents and brake fluids (Linden et al., 1985). Not until recently has butanol been used as a fuel additive for gasoline; prior to 2004, MTBE (mthyl tert-butyl ether), derived from isobutene, was the primary fuel additive to increase the octane rating of gasoline (Linden et al., 1985). When MTBE was banned in California due to adverse environmental impacts, butanol became a good candidate for fuel extension (Wittcoff et al., 2013). Now, (bio)butanol is used as a fuel additive for both gasoline and diesel fuel (Wittcoff et al., 2013).

(Bio)butanol has a low vapour pressure (see Table 2.2), low miscibility with water, and unlike (bio)ethanol, is completely miscible with diesel fuel and gasoline (Wittcoff et al., 2004). (Bio)butanol is less corrosive than (bio)ethanol, thus safer to handle, and can also be blended at the refinery with gasoline or diesel, thus (bio)butanol does not require separate transport and blending at the point of sale, like (bio)ethanol (Linden et al., 1985). (Bio)butanol blends in gasoline or diesel also do not require engine modifications as stoichiometric air-fuel ratio requirements are in line with that of gasoline and diesel (Linden et al., 1985) (see Table 1 in reference). Neat (bio)butanol has the potential to be a gasoline substitute because of (bio)butanol 
and gasoline's similar physical and chemical properties (e.g., lower heating value and octane number) found in Table 2.2. The octane rating of a fuel is the measure of compression a fuel can withsand before igniting based on a reference mixture of iso-octane and heptane. Higher octane ratings of fuels are more important for gasoline engines than diesel engines because the fuel is subject to compression prior to spark ignition, whereas diesel fuel ignites immediately from the increased temperature and pressure within the engine cylinder (Pasadakis et al., 2006). Neat (bio)butanol has never been used for transportation, but definitely, for reasons just mentioned, has the potential (Tashiro et al., 2013).

\subsubsection{Acetone}

Acetone and bio-acetone, like the alcohols previously discussed, are chemically interchangeable. Figure 2.2 depicts the chemical formula of (bio)acetone. Bio-acetone is rarely produced today. This is because the majority of acetone is produced as a by-product of phenol production (Howard, 2011). Phenol is produced by the following sequential processes: the alkylation of benzene by propene into cumene (isopropylbenzene), the partial oxidation of cumene and the cleavage of cumyl hydroperoxide to produce phenol and acetone in a 1.64:1 ratio (Juguin et al., 1990). Both reactants of the phenol production process, benzene and propene, are products of petroleum processing (Willcoff et al. 2004). Phenol is predominantly used as a precursor for plastics but also plays a role in drug and herbicide production (Chen et al., 2013). Since the demand for phenol and subsequent products is usually higher than the demand for acetone, producing acetone from any other method, including bio-methods, is inconsequential. Although bio-acetone can be produced by the fermentation of lignocelluosic material, with the predominance of the phenol production method, bio-acetone will likely not be produced unless there is added incentive to do so.

Acetone is biodegradable and is used as a solvent to produce the following, but not limited to, products: fats, oils, waxes, resins, rubber, plastics, lacquers and varnishes (Howard, 2011). Acetone is also used as an intermediate to make chemical compounds such as rayon, plastics, fibers, drugs, paint, varnish removers etc. (Hoffmeister et al., 2016; Howard, 2011). If acetone where produced by fermentation, subsequent product production using acetone has the potential to be more sustainable. The term "potential" is used here for good reason. Increasing a products 
sustainability involves not only taking into account the sustainability of the process used to create the product, but also the process used, to ensure the product is recycled or degraded into biodegradable material. For example, if bio-acetone is used to create plastic that is not biodegradable, than the sustainable production of acetone is arguably nullified.

\subsection{Biofuel impediments, government policy and government goals}

This section begins with a sub-section on the main impediments of biofuel production and use. This section concludes with current Canadian government biofuel policy and goals.

\subsubsection{Biofuel production impediments}

As discussed in section 2.1, mainstream society used biofuels as the primary fuel source before the 1950s rise of the petrochemical industry. Thus, an example of such an industry, on a smaller scale, is not a foreign concept and a lack of technology is not the reason for biofuel production impediment. North America was aware that anthropogenic carbon dioxide emissions would induce global warming in the 1960s (Oreskes, 2018). Thus, both the scientific and political community were aware in the 1970s that modifying the trajectory of the petrochemical industry and distribution system was the main impediment to a biofuel industry (Arbor, 1986). Consider, in the 1940s and 1950s, however, just after World War Two, many large petrochemical companies today, such as KOCH, were just beginning business development (U.S. Securities and Exchange Commission, 2005). The lack of political will to alter the economy and dissuade small business development is likely the cause of the political community's choice to overlook biofuels. This choice, in hindsight was irresponsible, but would have seemed completely understandable at the time. Similar public feelings associated with fostering small local business development are prominent today, and political figures are aware of public opinion.

Unfortunately, today, production chains that support traditional fuel production also support the production of several other chemicals, thus, making the conglomerate very difficult to modify now without significant investment and overall system modification. For example, the major objective of American refineries in the last 50 years has been to increase the octane rating of 
gasoline (Wittcoff et al. 2004). This objective gave rise to a multitude of different processes, including oligomerization, akylation and catalytic reforming (Schädlich et al. 2003). These processes are not only used in consort with crude oil distillation and refining but with several other product developments. Naphtha, one of the products of the crude oil distillation process, is subject to catalytic reforming to produce benzene, toluene and xylenes (Schadlich et al. 2003). Naphtha is also subject to anaerobic steam cracking to produce olefins such as propylene and ethylene (Schadlich et al. 2003). These products all play a role in the production of not only octane enhancing additives but in the production of plastics (e.g., polyethylene and polypropylene), synthetic fiber precursors (e.g., acrylonitrile) and industrial chemicals (e.g., glycols) (Willcoff et al. 2004; Schadlich et al. 2003). Products, such as gasoline, plastics, detergents, fibers, pesticides, tires, shampoo and sunscreen are based on seven raw materials derived from petroleum and natural gas: ethylene, propylene, $\mathrm{C} 4$ olefins (i.e., butenes and butadienes), benzene, toluene, xylenes and methane all using well established production processes (Wittcoff et al. 2013). Ninety five percent of the 500 billion pounds of chemical products produced each year come from the processes based on the production of these seven raw materials (Wittcoff et al. 2013).

\subsubsection{Canadian Policy}

Government policy has a large and significant impact on product development (Le Roy \& Klein, 2012). Neat RD is produced internationally and available in California because California's LCFS (Low Carbon Fuel Standard) guidelines, initiated in 2007, supports and mandates and increase in sustainable fuels (Cobb, 2015). First generation biofuels have seen incredible growth in the last decade largely due to policy goals (Seraj, 2014). In Canada, current biofuel production and consumption are highest in regions where government has played a large role in initiating and fostering relationships with the renewable energy industry (Taylor et al., 2005).

Current Canadian Federal government renewable fuel mandate, implemented in 2010 and modified in 2011, requires 5\% renewable contents in gasoline and 2\% renewable content in biodiesel sold in Canada (Government of Canada, 2017). On top of these federal regulations, provinces have created additional regulation to assist in the production and use of renewable fuels. For example, the Ontario Environmental Protection Act requires $4 \%$ total bio-based 
volume in fossil diesel, and requires a $70 \%$ reduction in emissions associated with the production of fossil diesel blends by 2017 (Cleantech Canada, 2014; Ontario Environmental Protection Act, 2014).

Even though these regulations are a step in the right direction towards reducing the GHG impact of the Canadian transport system, key exemptions such as fuel for aircraft, competition vehicles, trains, heating oil and military use as well as exemptions for some provinces make the federal and provincial regulations not as effective (Moorhouse \& Wolinetz, 2016). For example, Alberta's regulations do not apply to fuel produced and consumed within industrial operations, such as volumes of diesel fuels used in oil sand operations (Moorhouse \& Wolinetz, 2016). The exemptions have the potential to make policy GHG targets difficult to achieve.

Since the cost of producing or purchasing renewable content for both gasoline and petrol diesel (e.g., bioethanol and BD) is more than the cost of petrol diesel production, federal programs such as ecoEnergy of Biofuels, Next Gen Biofuels Fund and AAFC Growing Forward have been developed. These programs provide subsidies to Canadian biorefineries and suppliers to facilitate the renewable fuel mandate (Natural Resources Canada, 2015a). Some of these federal subsidies include production tax credits to support Canadian manufacture of BD, interest free loans and grants. The subsidies also target both the energy and agricultural sectors (small facilities) to support local ownership.

The overall effectiveness of these specific subsidies mentioned in the previous paragraph is still vague. Programs initiating these subsidies were developed in 2006 (Laan et al. 2009). Most of the programs listed above have ended in 2017 or are finishing in 2018 (Natural Resources Canada, 2015a). However, one way of measuring the cost effectiveness of these subsidies is by comparing the cost of the subsidies intended to prevent the use of carbon dioxide emissions with the amount it cost to purchase carbon offsets. The cost per ton of carbon dioxide equivalent offset is between \$4.23 US and \$33.83 US on the Chicago and European Climate Exchanges (Laan et al., 2009). From an economic perspective, it is thus 8 to 137 times more costly to avoid carbon dioxide emissions by producing biofuel (Laan et al., 2009). Comparatively, the way to measure a nation's overall GHG reduction effectiveness is by tracking GHG emissions and material use. A study completed by Moorhouse \& Wolinetz (2016) for Clean Energy Canada, found that Canada had increasingly reduced GHG emissions from 2011 to 2014 thus achieving a 
total of 4.3 MT of $\mathrm{CO}_{2} \mathrm{e}$ in 2014. This reduction in GHG emissions was calculated by using the 3.9 million cubic meters of renewable fuel Canadians used instead of fossil fuels that year (5\% of the total fuel use in Canada) (Moorhouse \& Wolinetz, 2016).

Ontario, the Canadian province with the largest population, has also created policies to reduce GHG emissions. More recently, Ontario passed the Climate Change Mitigation and Low Carbon Economy Act in 2016 and developed a Climate Change Action Plan (CCAP) adopted in 2017 that focuses on fighting climate change, reducing GHG emissions and transitioning to a low carbon economy (Ontario Ministry of the Environment and Climate Change, 2016). The cap and trade program, introduced in 2017 as part of the CCAP, puts a price on carbon. It is too early to determine the impact of the cap and trade program. It has been suggested that there could be some benefits to Ontario leaving the Western Climate Initiative (WCI) cap and trade program with California and opting for a carbon tax similar to British Columbia (Cameron, 2017). However, even if the Ontario government were to implement a carbon tax similar to the carbon tax in BC, Seraj (2014) indicates that unless this tax far exceeded BC's threshold tax rate, the tax would not create the incentive required to reduce fossil fuel use. Seraj (2014) indicates that the level of carbon tax required to perpetuate a shift in practice is around $\$ 2,000 / \mathrm{tCO}_{2} \mathrm{e}$. British Columbia's carbon tax is currently at its highest rate at $\$ 30 / \mathrm{tCO}_{2} \mathrm{e}$ (Seraj, 2014). Seraj (2014) argued that $\$ 30 / \mathrm{tCO}_{2} \mathrm{e}$ does not dissuade the use of fossil fuels nor does it make bio-fuel ventures economical (Seraj, 2014).

Presently, the focus of Ontario’s February 2018 CleanTech strategy as part of the CCAP prioritizes 1) energy generation and storage, 2) energy infrastructure, 3) bio-products and biochemicals and 4) water and wastewater (The Government of Ontario, 2018). The purpose of the strategy is to push Ontario's technology sector to develop more clean technology (i.e., sustainable technology); this includes less GHG intensive processes. By definition, clean technology is any process, product or service that reduces environmental impacts by reducing pollution (e.g., reducing GHG), more efficiently using natural resources and/or the use of significantly less energy than current industry standard (The Government of Ontario, 2018). Therefore, companies classified as clean technology companies need to fulfill at least one of these requirements. In order to foster the clean technology sector, the Ontario strategy focuses on developing programs and regulations that allow potential green companies to develop and 
thrive. The government of Ontario plans to leverage existing programs and develop new ones in order to improve information flow to new clean technology companies, provide access to needed capital and facilitate more efficient regulatory frameworks (The Government of Ontario, 2018).

\subsubsection{Canadian Government Goals}

Current renewable fuel regulations, implemented by the Canadian government in 2010, play a key role in supporting the current Canadian government's overall renewable fuel strategy (Government of Canada, 2017). The current Canadian government's sustainable technology development strategy supports the overall objective of reducing GHG emissions and this includes the development and use of biofuels (Natural Resources Canada, 2018). For example, Sustainable Development Technology Canada (SDTC) is a re-established organization funded by the Government of Canada to support CleanTech projects and coach fledging companies that meet CleanTech standards (Government of Canada, 2018). This organization would financially and socially support a company's project plans if this plan proves it reduces GHG impact compared to the process used to produce a pre-existing redundant function. The above mentioned regulations, strategy and programs support the Canadian 2015 goal of a reduction of 17\% GHG emissions from 2005 levels by 2020 (Natural Resources Canada, 2015a).

Canadian 2005 total GHG emissions was $738 \mathrm{MT} \mathrm{CO}_{2} \mathrm{e}$ (Environment and Climate Change Canada, 2015). Increases in GHG emissions between 1990 and 2015, according to Environment and Climate Change Canada, was due to an increase in mining, upstream oil and gas production and transport. Hence, Canadian $2015 \mathrm{GHG}$ emissions was not that much less at $722 \mathrm{MT} \mathrm{CO}_{2} \mathrm{e}$. A decrease in GHG emissions here was attributed to a reduction in public electricity and heat production utilities (Environment and Climate Change Canada, 2015). Thus, transport has not yet played a significant role in the reduction of Canada's GHG impact. If Canada is going to reach $17 \%$ of the $2005 \mathrm{GHG}$ level $\left(125 \mathrm{MT} \mathrm{CO}_{2} \mathrm{e}\right)$ by 2020 , a reduction of more than $16 \mathrm{MT}$ $\mathrm{CO}_{2} \mathrm{e} /$ year (given 738-722) is required and the transport sector should play a role in this reduction.

Canada also joined the Asia Pacific Partnership in 2005 with the intent of working with other nations and private sector companies to meet national goals for energy security, air pollution 
reduction and climate change reduction (Pond Technologies Inc., 2017). The focus of the partnership is to expand investment in and trade of sustainable technologies, specifically energy technologies including biofuels. Moorhouse \& Wolinetz (2016) indicated that in order for Canada to achieve substantial decarbonisation in support of international partnerships, biofuels would need to account for $20 \%$ of fuel use in Canada by 2030 and $90 \%$ by 2050 .

\subsection{Microalgae as a source of feedstock for biodiesel and ABE}

This section presents microalgae's basic characteristics that make it ideal for a biofuel feedstock. Also included is how best to use human waste streams to reduce the environmental impact of uncontrolled microalgal propogation, while at the same time enhancing microalgal growth in anthropogenic systems.

\subsubsection{Microalgae vs. Macroalgae}

There are estimated 300 thousand species of algae classified into two main groups: microalgae and macroalgae (Suganya et al., 2016). Microalgae are unicellular eukaryotic microphytes (i.e., aquatic plants) and by virtue of their name, contain membrane bound organelles with plastids that contain chlorophyll in order to carry out photosynthesis (Suganya et al., 2016). Figure 2.3 presents the basic organelles (e.g., lipid globules, lignocellulsic cell wall, protein) found in eukaryoic microalgae. Microalgae live in both fresh water and marine ecosystems (Suganya et al., 2016) and range is size between 3-30 $\mu \mathrm{m}$ (Molina Grima et al., 2003).

Macroalgae, also known as seaweed, are multicellular eukaryotic macrophytes that are also photosynthetically inclined and live in both fresh water and marine ecosystems (Suganya et al., 2016). Figure 2.4 depicts macroalgae with similar cellular components to microalgae presented in Figure 2.3. The cell walls and structural components that keep organelles in place in each cell contain most of the algae's lignocellulosic material. 


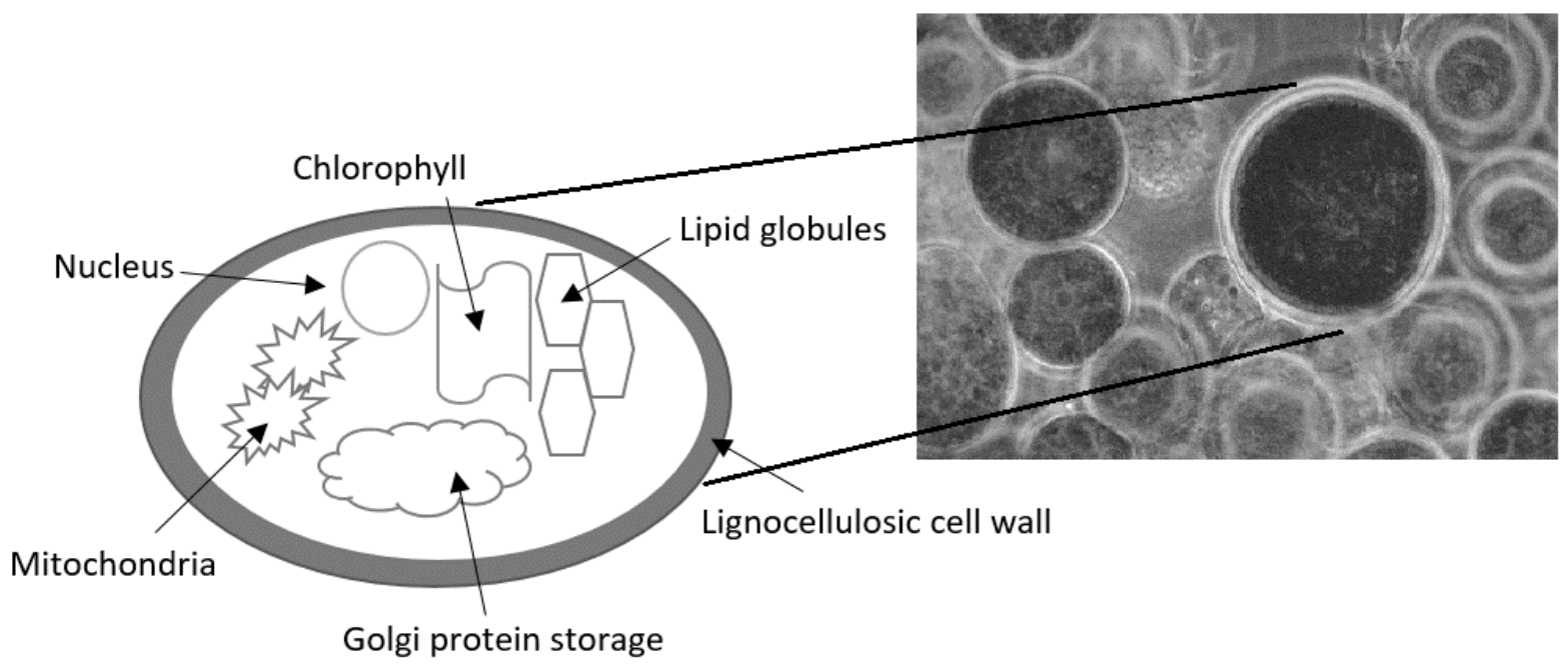

Figure 2.3: Basic components (i.e., organelles) of a eukaryotic microalgae (left), Microsopic photo (right), adapted from (FEBICO (Far East Bio-Tech. Co.) Ltd., 2018; Hammer \& Avalos, 2017)

Both micro and macroalgae have a much higher growth rate and $\mathrm{CO}_{2}$ fixation than any other plant on earth (Sander \& Murthy, 2010). The photosynthetic efficiency of algae is on average $13 \%$ whereas terrestrial plants have photosynthetic efficiencies of between one and two percent (Singh \& Ahluwalia, 2013). Both micro and macroalgae can also accumulate high levels of lipids. As biodiesel predominantly consists of mono-alkyl esters of long chain fatty acids, both macro and microalgae are ideal for biodiesel feedstock (Sharara et al., 2012; Wiley et al., 2011) There are limited reports concerning the production of biofuels from macroalgae (Suganya et al., 2016). It is suspected that higher production costs and harvesting difficulties make using macroalgae less appealing in general and less appealing than microalgae (Brennan \& Owende, 2010). Suganya et al. (2016) indicate that growing macroalgae is suited to a limited area such as coastline in relatively stagnant waters. Harvesting a reliable quantity is also difficult because of climatic variability (Suganya et al., 2016). There is also the possibility that microalgae has been studied more because it has been grown in small capacities for niche markets (i.e., pharmaceutical and aquiculture) (Suganya et al., 2016). 


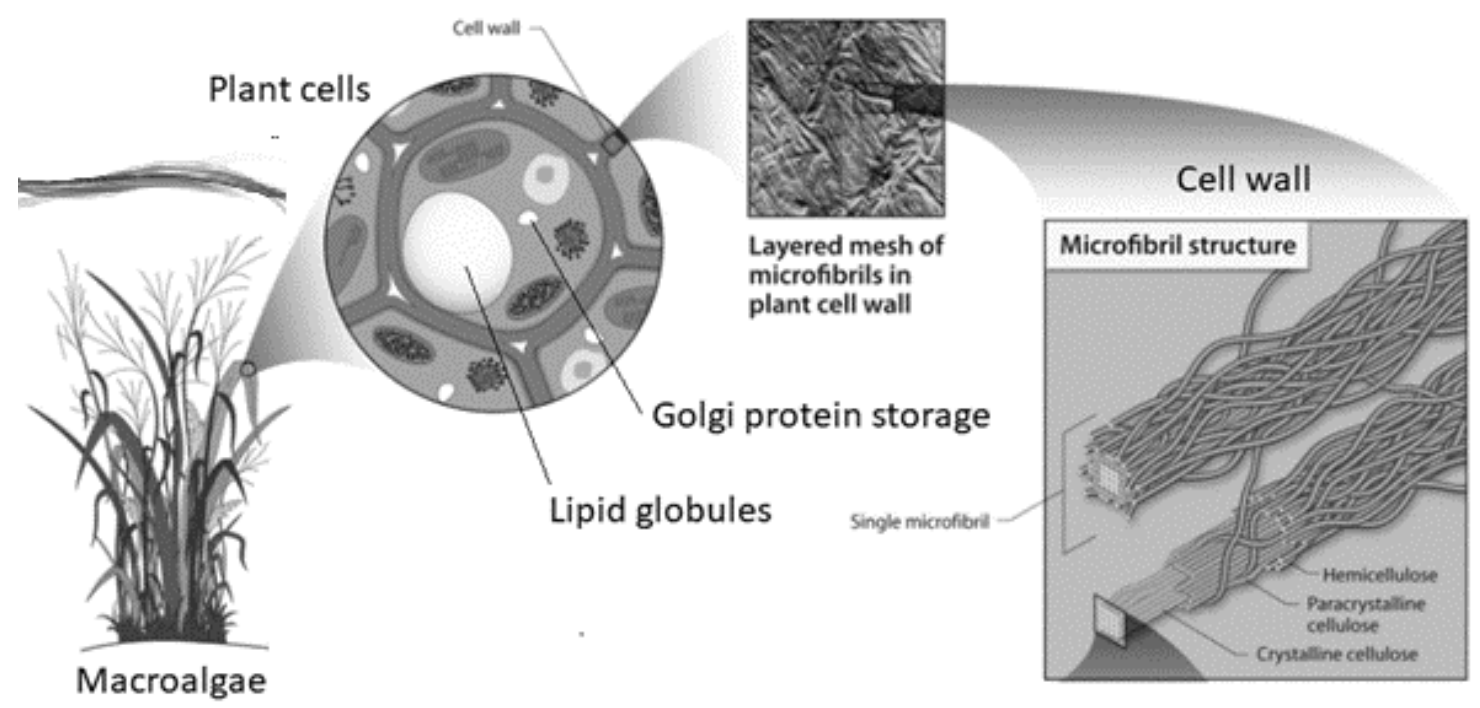

Figure 2.4: Macroalgae from plant to cellular level, adapted from (Quiroz-Castañeda \& FolchMallol, 2013)

\subsubsection{Microalgal physical makeup}

Microalgae contain carbohydrates, lipids and proteins. Carbohydrates, also called "lignocellulosic biomass", make up most of microalgae's physical structure. Lignocellulosic biomass in microalgae consist of cellulose, hemicellulose and lignin (see Figure 2.5). For biofuel production, cellulosic and hemicellulosic biomass are broken down into monomers and fermented to produce alcohols (Mu et al., 2010; Sheehan et al., 2003).

For this study's purpose, lipids in microalgae are either triglycerides (TG), fatty acids (FA) or free fatty acids (FFA). Triglycerides are composed of one, two or three FAs attached to a glycerol backbone as shown in Figure 9. A transesterification reaction converts triglycerides to FAME (also known as BD). Therefore, one of many characteristics sought after in microalgae for $\mathrm{BD}$ production is TG content. 


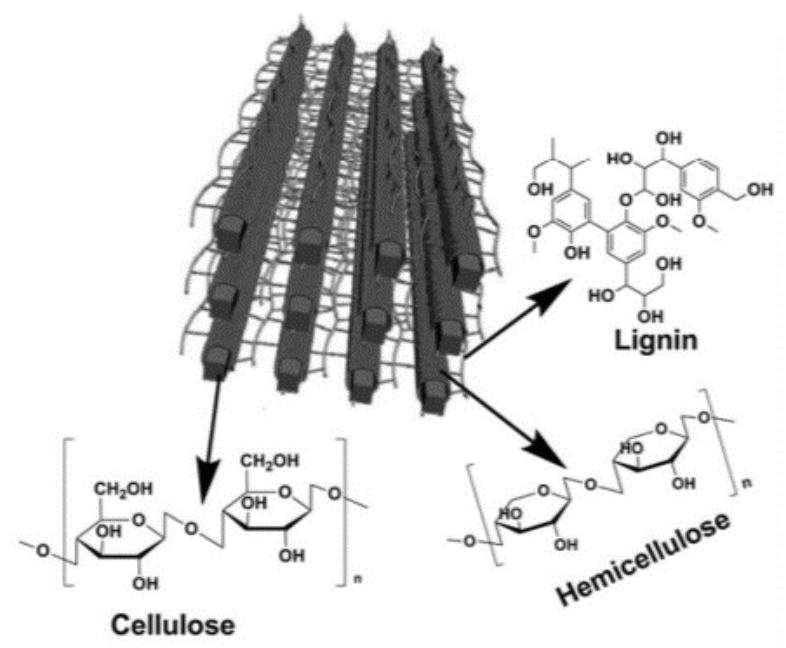

Figure 2.5: Schematic of the location and structure of lignin (web), hemicellulose (casing) and cellulose (inside hemicellulose) in lignocellulosic biomass, adapted from (Liu et al., 2015)

Fatty acids are carboxylic acids that have long hydrocarbon chains (normally between 4 and 22 carbons in length) with a carboxylic acid group at one end (Cabus-Llaurado et al., 2007). The basic form of a fatty acid has a hydrocarbon chain completely saturated with hydrogen (i.e., no double bonds). When double bonds are present in the fatty acid hydrocarbon chain, either one or more, these fatty acids are called monounsaturated fatty acids (MUFAs) or polyunsaturated fatty acids (PUFAs) respectively (see Figure 2.6). Saturated fatty acids make superior biodiesel thus, algal species for use as biomass for biodiesel production are selected accordingly (Patil et al., 2012). The most abundant saturated fatty acid in microalgae is palmitic acid (16:0), followed by stearic acid (18:0), while of the monounsaturated types, oleic acid proves most abundant (18:1n 9) (Martinez et al., 2000). The ratios represent the nomenclature used for the fatty acids also shown in Figure 2.6.

FFAs are lone FAs not part of a triglyceride. Microalga with an inordinate amount of FFA are not suitable for BD production, however an acid pre-treatment can facilitate BD production from these lipids as well (Dong et al., 2016). 


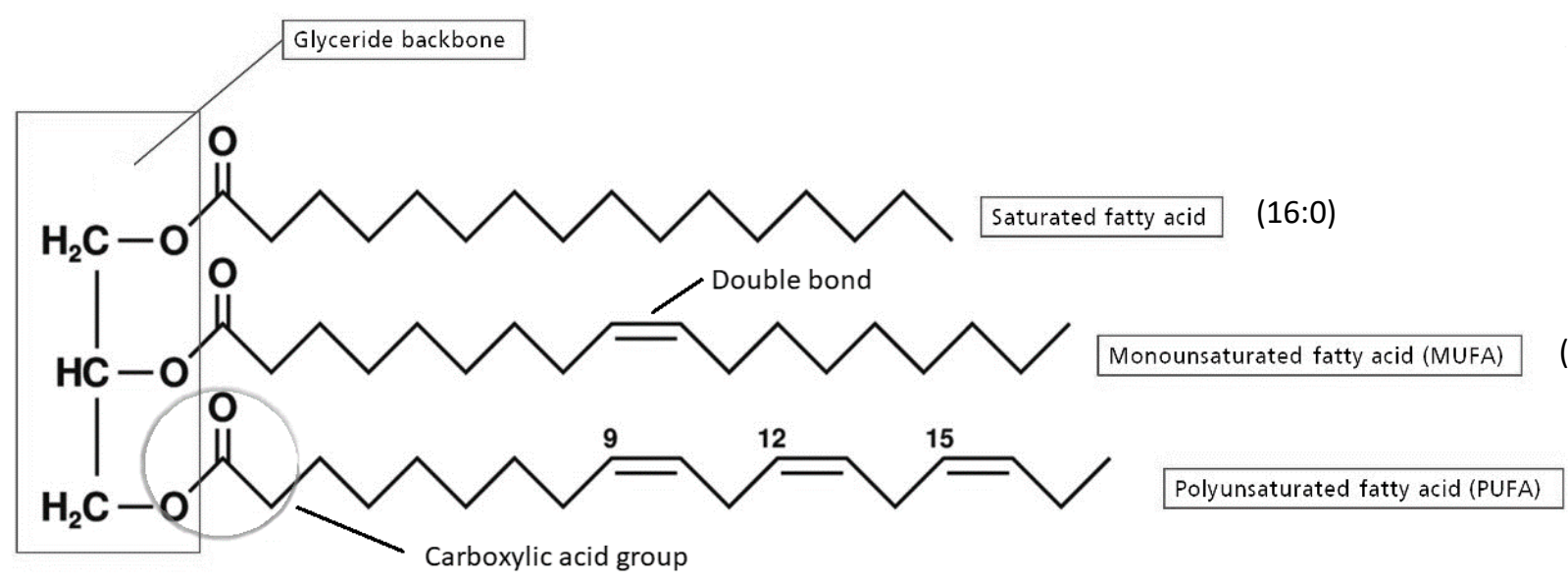

$(18: 1 n 9)$

Figure 2.6: Triglyceride consisting of three fatty acids and glycerol backbone, carboxylic acid group is circled, adapted from (Oxford Instruments, 2018)

\subsubsection{Algae found suitable for biofuel processing}

Microalgae have varying ratios of lignocellulosic biomass, proteins and lipids depending on the genus (taxonomic level) and the environment in which they grow. Table 2.3 below gives examples of the main algal taxonomic divisions. There are over 40 thousand different known species of algae and new species are discovered on a regular basis (Suganya et al., 2016).

Algae taxonomic groups contain several algae genera. Each algal genus may have several species. Research tends to refer to different algal species not only by their species name but also by their batch numbers, which facilitates research reproducibility (Yun et al., 1997). The microgalae species found in Table 2.4 are numbered based on their corresponding taxonomic division in Table 2.3. Not all microalgal genera could be linked to a taxonomic division, therefore it should be assumed that microalgal genus in Table 2.4 may or may not be associated with a taxonomic division in Table 2.3. Table 2.4 also outlines the microalgae species percent composition of carbohydrates, proteins and lipids. For biofuel (i.e., biodiesel and alcohol) production, microalgal biomass must have relatively high lipid and lignocellulosic content. Microalgae species can be forced, with varying degrees of success, to produce higher lipid yields when grown in a medium with a limiting amount of nitrogen (Ratledge, 2004). It is quite common, in such nitrogen deficient circumstances, to see some microalgae produce up to $50 \%$ oil content (Chisti, 2007; Suganya et al., 2016). However, productivity and lipid accumulation 
are inversely related (Hu et al., 2008). A balance is required in order to increase lipid yield but only to the extent that this increase does not substantially slow down overall biomass growth. Mata et al. (2010) contains a comprehensive breakdown of lipid content, lipid productivity and biomass productivity for marine and freshwater microalgae.

Table 2.3: Taxonomic groups of algae and their common names- adapted from (Suganya et al., 2016)

\begin{tabular}{|l|l|l|l|}
\hline $\begin{array}{l}\text { Reference } \\
\text { Number }\end{array}$ & $\begin{array}{l}\text { Taxonomic groups } \\
\text { (divisions) }\end{array}$ & Best known as & $\begin{array}{l}\text { Nuclear } \\
\text { characteristics }\end{array}$ \\
\hline 1 & Bacillariophyta & Diatoms & Eukaryote \\
\hline 2 & Chloro phycophyta & Green algae & Eukaryote \\
\hline 3 & Chrysophycophyta & Golden algae & Eukaryote \\
\hline 4 & Cyanobacteria & Blue green algae & Prokaryote \\
\hline 5 & Phaeco phycophyta & Brown algae & Eukaryote \\
\hline 6 & Dinophyta & Dinoflagellates & Mesokaryote \\
\hline 7 & Rhodo phycophyta & Red algae & Eukaryote \\
\hline 8 & Euglenoids & & Mesokaryote \\
\hline 9 & Cryptophyta & & Eukaryote \\
\hline 10 & Haptophyta & & Eukaryote \\
\hline 11 & Xanthophyta & Yellow green algae & Eukaryote \\
\hline 12 & Raphidiophyta & Chloromonads & Eukaryote \\
\hline
\end{tabular}

As can be seen in Table 2.5, microalgae found the most suitable for feedstock biodiesel processing are species of the genera Nannochloropsis, Chlorella and Scenedesmus (also shaded in Table 2.4). These genera naturally have high lipid content (Chisti 2007; Liu et al. 2017; Patil et al. 2012; Sharara et al. 2012). Cristi (2007) found Chlorella sp. and Nannochloropsis sp. to have oil content based on percent dry weight of between $28 \%$ and $68 \%$, and thus suitable for biodiesel production. These same genera also have relatively high biomass productivities (growth rate) as shown in Table 2.5. Pond Technologies Inc. in Markham Ontario is currently using Chlorella for biofuel, biolubricant and biochemical production (Pond Technologies Inc., 2017). Pond Technologies does not disclose the specific species used. 
Table 2.4: Microalgal genus and their compositions

\begin{tabular}{|c|c|c|c|c|c|}
\hline $\begin{array}{l}\text { Reference } \\
\#\end{array}$ & Microalgal genus & $\begin{array}{l}\text { Oil content } \\
(\% \text { dry wt })\end{array}$ & $\begin{array}{l}\text { Carbohydrate } \\
\text { (\% dry wt) }\end{array}$ & $\begin{array}{l}\text { Protein } \\
\text { (\% dry } \\
\text { wt) }\end{array}$ & Reference \\
\hline 2 & $\begin{array}{l}\text { Botryococcus } \\
\text { braunii }\end{array}$ & $25-75$ & & & (Chisti, 2007) \\
\hline 2 & Chlorella & $18.0-57.0$ & & & (Mata et al., 2010) \\
\hline 2 & Chlorella emersonii & $25.0-63.0$ & & & (Mata et al., 2010) \\
\hline 2 & $\begin{array}{l}\text { Chlorella } \\
\text { protothecoides }\end{array}$ & $14.6-57.8$ & & & (Mata et al., 2010) \\
\hline 2 & $\begin{array}{l}\text { Chlorella } \\
\text { protothecoides }\end{array}$ & 55.2 & 15.0 & & (Miao \& Wu, 2006) \\
\hline 2 & $\begin{array}{l}\text { Chlorella } \\
\text { sorokiniana }\end{array}$ & $19.0-22.0$ & & & (Mata et al., 2010) \\
\hline 2 & Chlorella sp. & $28-32$ & & & (Chisti, 2007) \\
\hline 2 & Chlorella sp. & $10.0-48.0$ & & & (Mata et al., 2010) \\
\hline 2 & $\begin{array}{l}\text { Chlorella } \\
\text { syrenoidosa }\end{array}$ & 2.0 & & & (Mata et al., 2010) \\
\hline 2 & Chlorella vulgaris & $14-22$ & $12-17$ & $51-58$ & (Suganya et al., 2016) \\
\hline 2 & Chlorella vulgaris & $22.1 \pm 2.0$ & & & (Sobczuk et al.. 2008) \\
\hline 2 & Chlorella vulgaris & $5.0-58.0$ & & & (Mata et al., 2010) \\
\hline 6 & $\begin{array}{l}\text { Crypthecodinium } \\
\text { cohnii }\end{array}$ & 20 & & & (Chisti, 2007) \\
\hline 1 & Cylindrotheca sp. & $16-37$ & & & (Chisti, 2007) \\
\hline 2 & $\begin{array}{l}\text { Dunaliella } \\
\text { primolecta }\end{array}$ & 23 & & & (Chisti, 2007) \\
\hline \multirow[t]{2}{*}{2} & Dunaliella $s p$. & $17.5-67.0$ & & & (Mata et al., 2010) \\
\hline & Euglena gracilis & $14-20$ & $14-18$ & $39-61$ & (Suganya et al.. 2016) \\
\hline 11 & Monallanthus salina & $>20$ & & & (Chisti, 2007) \\
\hline 2 & Nannochloris sp. & $20-35$ & & & (Chisti 2007) \\
\hline \multirow[t]{4}{*}{2} & Nannochloris sp. & $20.0-56.0$ & & & (Mata et al., 2010) \\
\hline & $\begin{array}{l}\text { Nannochloropsis } \\
\text { oculata }\end{array}$ & $22.7-29.7$ & & & (Mata et al., 2010) \\
\hline & Nannochloropsis sp. & $31-68$ & & & (Chisti, 2007) \\
\hline & Nannochloropsis sp. & $12.0-56.0$ & & & (Mata et al., 2010) \\
\hline 2 & $\begin{array}{l}\text { Neochloris } \\
\text { oleoabundans }\end{array}$ & $35-54$ & & & (Chisti, 2007) \\
\hline 1 & Nitzschia sp. & $45-47$ & & & (Chisti, 2007) \\
\hline \multirow[t]{2}{*}{1} & $\begin{array}{l}\text { Phaeodactylum } \\
\text { tricornutum }\end{array}$ & $20-30$ & & & (Chisti, 2007) \\
\hline & Prymnesium parvum & $22-39$ & $25-33$ & $28-45$ & (Suganya et al., 2016) \\
\hline 2 & $\begin{array}{l}\text { Scenedesmus } \\
\text { dimorphus }\end{array}$ & $16-40$ & $21-52$ & $8-18$ & (Suganya et al., 2016) \\
\hline 2 & Scenedesmus acutus & 40 & 40 & 20 & (Dong et al., 2016) \\
\hline 2 & $\begin{array}{l}\text { Scenedesmus } \\
\text { obliquus }\end{array}$ & $12-14$ & $10-17$ & $50-56$ & (Suganya et al., 2016) \\
\hline 2 & $\begin{array}{l}\text { Scenedesmus } \\
\text { obliquus }\end{array}$ & $11.0-55.0$ & & & (Mata et al., 2010) \\
\hline
\end{tabular}




\begin{tabular}{|l|l|l|l|l|l|}
2 & $\begin{array}{l}\text { Scenedesmus } \\
\text { quadricauda }\end{array}$ & $1.9-18.4$ & & & (Mata et al., 2010) \\
\hline 2 & Scenedesmus sp. & $19.6-21.1$ & & & (Mata et al., 2010) \\
\hline & Schizochytrium sp. & $50-77$ & & & (Chisti, 2007) \\
\hline & Spirogyra sp. & $11-21$ & $33-64$ & $6-20$ & (Suganya et al., 2016) \\
\hline
\end{tabular}

Cyanobacteria are between 0.2 and $100 \mu \mathrm{m}$ in size and can also accumulate high levels of lipids and lignocellulosic biomass (Chorus \& Bartram, 1999; Karatay \& Dönmez, 2011; Tonietto et al., 2014). Most microalgae accumulate lipids as storage when under stress, whereas cyanobacteria accumulate lipids in thylakoid membranes when they are the most photosynthetically productive and increasing biomass (Karatay \& Donmez, 2011).

Table 2.5: Microalgal biomass productivity

\begin{tabular}{|l|l|l|}
\hline Microalgae & Growth rate & Reference \\
\hline Nannochloropsis $\mathrm{sp}$. & $0.27 \mathrm{~g} / \mathrm{L} \cdot$ day & (Brennan \& Owende, 2010) \\
\hline Chlorella sorokiniana & $1.47 \mathrm{~g} / \mathrm{L} \cdot$ day & (Brennan \& Owende, 2010) \\
\hline Chlorella & $3.8 \mathrm{~g} / \mathrm{L} \cdot$ day & (Brennan \& Owende, 2010) \\
\hline Botryococcus sp. & $1.1 \mathrm{~g} / \mathrm{L} \cdot$ day & (Murakami \& Ikenouchi, 1997) \\
\hline Chorella vulgaris & $0.26 \mathrm{~g} / \mathrm{L} \cdot$ day & (Ma, 2016) \\
\hline Scenedesmus sp. & $0.194-0.248 \mathrm{~g} / \mathrm{L} \cdot$ day & (Zhou et al., 2011) \\
\hline Nannochloropsis & $0.51 \mathrm{~g} / \mathrm{L} \cdot$ day & (Liu et al., 2017) \\
\hline Nannochloropsis & $1-3 \mathrm{doubling} /$ day & (Patil et al., 2012) \\
\hline unspecified & $1.535 \mathrm{~g} / \mathrm{L} \cdot$ day & (Chisti, 2007) \\
\hline
\end{tabular}

Karatay and Donmez (2011) studied cyanobacteria lipid content and found large lipid content (\% dry mass) as well as strong FAME yield from the cyanobacteria genus shown in Table 2.6.

Cyanobacteria can be found in freshwater lakes such as Lake Erie where there was a significant cyanobacteria bloom in 2012 (Scavia et al., 2014; Suganya et al., 2016). According to Conroy et al. (2017), Cyanobacterium cylindrospermopsis is likely the ongoing predominant cyanobacteria strain found in lake Erie and will most likely remain so as lake temperatures rise and water levels fall (Conroy et al., 2007). 
Table 2.6: Lipid content and FAME conversion for cyanobacteria genus (Karatay \& Donmez, 2011)

\begin{tabular}{|l|l|l|l|}
\hline $\begin{array}{l}\text { Cyanobacteria } \\
\text { Genus }\end{array}$ & $\begin{array}{l}\text { Maximum lipid } \\
\text { content (\% dry } \\
\text { weight) }\end{array}$ & $\begin{array}{l}\text { C16 and C18 methyl } \\
\text { ester yield (as a \% of } \\
\text { lipid content) }\end{array}$ & $\begin{array}{l}\text { Saturated lipid } \\
\text { content (\% of lipid } \\
\text { content) }\end{array}$ \\
\hline Synechococcus sp. & 42.8 & 46.9 & 74.5 \\
\hline $\begin{array}{l}\text { Cyanobacterium } \\
\text { aponinum }\end{array}$ & 45.0 & 67.7 & 77.9 \\
\hline Phormidium $\mathrm{sp}$. & 38.2 & 90.6 & 84.7 \\
\hline
\end{tabular}

Sourcing algae from natural freshwater can reduce costs associated with exclusively growing algae (Ma, 2016). Cyanobacteria size also naturally lend itself to a relatively cost effective and energy efficient microscreen harvest method which could make this microalgae easier to extract from natural water bodies (Brennan \& Owende, 2010). Extensive algal growth in fresh water lakes is not something to perpetuate for the want of microalgal biomass for fuel production, however, temporarily removing toxic algae from a lake while simultaneously benefiting biofuel production has its merits.

Regardless, there has been limited mention of cyanobacteria use as a biofuel source. Karatay \& Donmez (2011) suggested that biodiesel produced from cyanobacteria biomass would have a high $\mathrm{CN}$ and high oxidative stability due to high percent saturation, which are important advantage for fuel performance and storage capability. However, there is no other literature that mention current biofuel production from cyanobacteria.

2.3.4 Enhancing microalgal growth while optimizing waste recycling and carbon sequestration

Flue Gas

Certain microalgae have the tolerance for and can thrive in an environment with high $\mathrm{CO}_{2}$ concentrations (Bhola et al., 2014). Carbon dioxide is a food source for the algae that requires $\mathrm{CO}_{2}$ to complete photosynthesis and grow. Thus, alga, used in this way, is a $\mathrm{CO}_{2}$ sequestration tool. Between 10 and 15 percent $\mathrm{CO}_{2}$ concentration produces maximum biomass for most microalgal species (Bhola et al., 2014; Singh \& Ahluwalia, 2013). Industrial flue gas $\mathrm{CO}_{2}$ 
concentrations currently released into the atmosphere range from 7 to 20\% (Bhola et al., 2014). It is essential to select strains of algae that can grow effectively and accumulate biomass in industrial flue gas (emissions), as this is where most of the carbon sequestration is required (Singh et al. 2011; Suganya et al. 2016).

Table 2.7 outlines growth rates associated with varying concentrations of $\mathrm{CO}_{2}$. All microalgae in Table 2.7 are eukaryotic green microalgae except for Spirulina sp., which is a prokaryotic cyanobacterium. Most species are of the green algae genus because the decreased $\mathrm{pH}$ in the culture medium caused by high concentration of $\mathrm{CO}_{2}$ is favorable for green algae (Murakami \& Ikenouchi, 1997).

Table 2.7: Microalgae subject to carbon dioxide emissions

\begin{tabular}{|c|c|c|c|}
\hline Microalgae & Growth rate / Other & $\mathrm{CO}_{2}$ concentration & Reference \\
\hline Spirulina sp. & $0.2 \mathrm{~g} / \mathrm{L} \cdot \mathrm{day}$ & $6 \%$ & $\begin{array}{l}\text { (De Morais \& Costa } \\
\text { 2007) }\end{array}$ \\
\hline Chlorella sp. & $\begin{array}{l}0.24 / \mathrm{hr} \text { (doubling in } \\
\text { less than } 3 \text { hours) }\end{array}$ & $20 \%$ at $40^{\circ} \mathrm{C}$ & (Sakai et al., 1995) \\
\hline Chlorella sp. & Fixed $1 \mathrm{gCO}_{2} / \mathrm{L} \cdot$ day & $15 \%$ at $35^{\circ} \mathrm{C}$ & $\begin{array}{l}\text { (Murakami \& } \\
\text { Ikenouchi, 1997) }\end{array}$ \\
\hline Chlorella sp. & $\begin{array}{l}\mathrm{NOx} \text { at } 45 \mathrm{mg} / \mathrm{m}^{3} \text { and } \\
\mathrm{CO} \text { at } 3 \mathrm{mg} / \mathrm{m}^{3} \text { did } \\
\text { not affect growth }\end{array}$ & $6-8 \%$ & (Doucha et al., 2005) \\
\hline Botryococcus braunii & $\begin{array}{l}0.027 \mathrm{~g} / \mathrm{L} \cdot \text { day }(21 \% \\
\text { lipid) }\end{array}$ & $10 \%$ & (Yoo et al., 2009) \\
\hline Botryococcus sp. & $1.1 \mathrm{~g} / \mathrm{L} \cdot$ day & Not stated & $\begin{array}{l}\text { (Murakami \& } \\
\text { Ikenouchi, 1997) }\end{array}$ \\
\hline Senedesmus obliquus & $0.14 \mathrm{~g} / \mathrm{L} \cdot \mathrm{day}$ & $12 \%$ & $\begin{array}{l}\text { (De Morais \& Costa } \\
\text { 2007) }\end{array}$ \\
\hline
\end{tabular}

Microalgae can also grow well in a range of temperatures, thus reducing the need for substantial cooling of the flue gas. Additionally, microalgae can grow with moderate levels of nitrogen dioxide and sulfur dioxides present, which are common elements in flue gas (Singh \& Ahluwalia, 2013). Brennan \& Owende (2010) found that flue gas can actually control algal invasive species to a certain extent because only certain strains can survive in high $\mathrm{CO}_{2}$ concentrated flue gas environments. 


\section{Wastewater}

In order to reduce the cost and environmental impact of using processed water for microalgal growth, wastewater should be used instead (Ferrell \& Reed, 2010). Growing microalgae in wastewater benefits both the biorefinery that will produce the microalgal biofuel and the wastewater treatment facility (Singh et al., 2011; Wang et al., 2008; Wiley et al., 2011). First, the effective use of wastewater eliminates the need for large amounts of additional freshwater to grow microalgae. Second, wastewater also has significant amounts of phosphorus, nitrogen and ammonia, which eliminates the environmental impact associated with fertilizer production.

This study looked at the wastewater treatment plant in Barrie Ontario for reference purposes. Figure 2.7 is an aerial photo of the Barrie wastewater treatment plant. The wastewater is pumped to raised tanks (1) and then moves to the primary clarifiers (2), where most of the large solids precipitate out of solution. Pure oxygen supplies and saturates the wastewater in the aeration tanks (3) and steady mixing allows the bacteria culture to break down the organic material in the wastewater releasing phosphorus and ammonia into solution. It is here from the secondary clarifiers where wastewater should be drawn to support microalgal growth as it is here where phosphorus and nitrogen are readily available for uptake by microalgal cells (Sen et al., 2013).

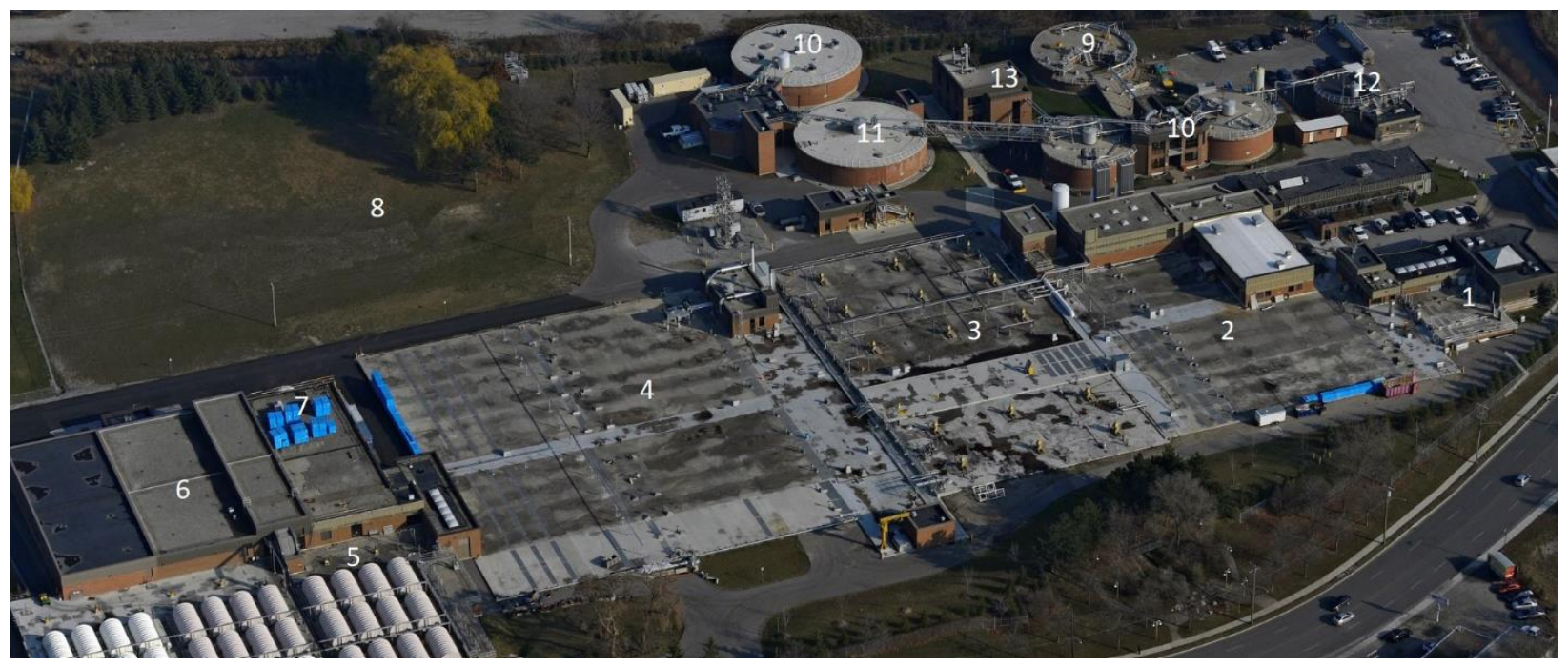

Figure 2.7: Barrie wastewater treatment plant layout 2017: 1 - Pump house, 2 - Primary clarifiers, 3 - Aeration tanks, 4 - Secondary clarifiers, 5 - Rotating biological contactors, 6 Sand filter, 7 - UV treatment, 8 - Clean water discharge piping, 9 - Aerobic digester, 10 Primary digesters, 11 - Secondary digester, 12 - Biosolid holding tank, 13 - Co-generation facility (City of Barrie, 2004) 
Table 2.8 below includes the growth rate and nutrient removal rates of some microalgae using wastewater. Kong et al. (2010) experimented with nutrient balancing and found that adding nutrients improved the growth rate of Chlamydomonas reinhardtii overall. Table 2.8 growth rates vary, however, the removal of the majority of phosphorus and nitrogen is more consistent.

Table 2.8: Microalgae grown in wastewater

\begin{tabular}{|c|c|c|c|}
\hline Microalgae & Growth rate & Removal of nutrients & Reference \\
\hline $\begin{array}{l}\text { Scenedesmus } \\
\text { obliquus }\end{array}$ & $1.0 \mathrm{mg} / \mathrm{L} \cdot \mathrm{day}$ & $\begin{array}{l}35.5 \% \text { decrease in BOD with } \\
100 \% \text { recycled water from } \\
\text { an olive oil extraction plant }\end{array}$ & (Hodaifa et al. 2007) \\
\hline $\begin{array}{l}\text { Scenedesmus } \\
\text { obliquus }\end{array}$ & $\begin{array}{l}0.77 \mathrm{mg} / \mathrm{L} \cdot \text { day at } \\
25^{\circ} \mathrm{C}\end{array}$ & $\begin{array}{l}98 \% \text { removal of } \mathrm{P} \text { and } 100 \% \\
\text { removal of } \mathrm{N} \text { in stirred } \\
\text { culture with HRT of } 4 \text { days }\end{array}$ & $\begin{array}{l}\text { (Aslan \& Kapdan, } \\
\text { 2006) }\end{array}$ \\
\hline $\begin{array}{l}\text { Scenedesmus } \\
\text { obliquus }\end{array}$ & $\begin{array}{l}68 \% \text { oil and } 16 \% \\
\text { lignocellulosic }\end{array}$ & $\begin{array}{l}\text { Synthetically created } \\
\text { wastewater used }\end{array}$ & $\begin{array}{l}\text { (Martinez et al., } \\
\text { 2000) }\end{array}$ \\
\hline Chorella vulgaris & $0.624 \mathrm{~g} / \mathrm{L} \cdot$ day & $\begin{array}{l}\text { Removal of } 0.92 \mathrm{~g} \\
\mathrm{NH}_{3} / \mathrm{m}^{3} \cdot \mathrm{hr} \text { supplemented } \\
\text { with } 46 \mathrm{~g} \mathrm{PO}_{4}^{-3} / \mathrm{m}^{3} \text { using } \\
\text { wastewater from a steel plant } \\
\text { and } 15 \%(\mathrm{v} / \mathrm{v}) \mathrm{CO}_{2}\end{array}$ & (Yun et al., 1997) \\
\hline Chorella sp. & Not specified & $\begin{array}{l}\text { Removal of } 93.3 \% \mathrm{NH}_{3}, \\
89.1 \% \mathrm{TN}, 80.9 \% \mathrm{TP} \text { from } \\
\text { municipal wastewater }\end{array}$ & (Chen et al. 2015) \\
\hline $\begin{array}{l}\text { Chlamydomonas } \\
\text { reinhardtii }\end{array}$ & $\begin{array}{l}2.0 \mathrm{~g} / \mathrm{L} \cdot \text { day at } \\
\text { lipid content of } \\
25.25 \%(\mathrm{w} / \mathrm{w})\end{array}$ & $\begin{array}{l}\text { Removal of } 55.8 \mathrm{mg} / \mathrm{L} \cdot \text { day } \\
\text { of TN and } 17.4 \mathrm{mg} / \mathrm{L} \cdot \text { day of } \\
\text { TP removed from municipal } \\
\text { wastewater }\end{array}$ & $\begin{array}{l}\text { (Kong, Li, Martinez, } \\
\text { Chen, \& Ruan, 2010) }\end{array}$ \\
\hline
\end{tabular}

The correct ratio of nitrogen, phosphorus and carbon is one of the most important nutrient characteristics for optimal microalgal growth (Christenson \& Sims, 2011). Optimal C:N:P ratio for microalgal growth is approximately 100:11:1 (Chisti, 2007). Municipal wastewater typically has between 20 and $50 \mathrm{w} \%$ organic carbon (Torri et al. 2014), $120.6-530 \mathrm{mg} / \mathrm{L}$ of total phosphorus and 128.6 - $290 \mathrm{mg} / \mathrm{L}$ of total nitrogen (Kong et al., 2010; Min et al., 2011). Ma (2016) used levels of total nitrogen and phosphorus of $141 \mathrm{mg} / \mathrm{L}$ and $178 \mathrm{mg} / \mathrm{L}$ respectively (Ma, 2016). Therefore, the microalgal culture does require additional nitrogen and carbon by means of nutrient recycling to assist with optimal nutrient concentrations for optimal microalgal growth. 
Another option for microalgal growth of even greater benefit to the wastewater treatment facility is the use of microalgae as part of the wastewater treatment process. Instead of using the wastewater for microalgal growth, with the understanding that the wastewater will return and be further treated using the wastewater treatment plant, microalgae can also be used as a primary source of wastewater treatment. Biological wastewater treatment systems use microalgae as part of the wastewater treatment process. Biological wastewater treatment systems have increased in importance in the last 50 years and are now accepted as an effective means of treating wastewater (Sen et al., 2013). However, these plants still have the primary purpose of wastewater treatment and not necessarily the dual objective of producing clean water and significant amounts of microalgal biomass. Effective wastewater treatment in consort with biomass production for biofuel has yet to be demonstrated economically feasible (Christenson \& Sims, 2011). One of the reason why the collaboration potential between the biofuel industry and the wastewater industry has not yet been realized is because those testing algae production technologies have not often integrated their research with that of the wastewater industry and vice versa (Christenson \& Sims, 2011).

The Barrie wastewater treatment plant does not use biological wastewater treatment, but does use aerobic and anaerobic digesters along with a co-generation facility. The Barrie wastewater treatment plant provides wastewater treatment to a city of approximately 285 thousand people. Sewers are directed to the plant, however, road run off is not. Using the Barrie wastewater treatment plant as a reference, this study calculated the approximate amount of microalgae growth achievable in a year given the total amount of wastewater produced by the city. At an approximate wastewater production of 403 liters per person per day (City of Barrie, 2004), a city the size of approximately 285,000 people would produce just under enough wastewater to produce 10,000 tonnes of microalgae per year given a microalgal growth rate of $265 \mathrm{mg} / \mathrm{L} \cdot$ day. Ten thousand tonnes of microalgae is equivalent to approximately 3,400 tonnes or 4 million liters of biodiesel.

Mixed strain use

Studies suggest using multiple strains of microalgae in the overall biofuel production process especially if using municipal wastewater as a source of water and nutrients (Awudu \& Zhang, 
2011). Monocultures of high lipid producing strains are likely to be outcompeted by faster growing species of microalgae or cyanobacteria (Vasudevan \& Briggs, 2008). In wastewater treatment plants, naturally occurring mixed cultures dominate. Wastewater might already contain some algal growth, which would contaminate the monoculture of a PBR if monocultures were attempted. Also, if microalgae were used to assist in the wastewater treatment process, the literature is predominantly focused on mixed algal strain systems (Christenson \& Sims, 2011).

Lipid yield of mixed cultures in wastewater has achieved 11.3\% lipid and as high as $29 \%$ when grown in anaerobic digester effluent (Woertz et al., 2009). Griffiths (2009) found FAME content of $23.4 \%$ after in-situ transesterification of mixed culture gown in municipal wastewater (Griffiths, 2009). Farooq et al. (2013) found that the yield of BD from extracted algal oil using a mixed microalgal culture was upwards of $92 \%$ (Farooq et al., 2013). There is therefore potential for successful microalgal growth using wastewater, and subsequent oil extraction, with a variety of microalgal genera grown concurrently in a mixed culture.

\section{Mixotrophic conditions}

When growing microalgae in wastewater, a carbon nutrient source, usually glucose or glycerol, is required to balance the $\mathrm{C} / \mathrm{N}$ ratio to promote algal growth. Summarized in Table 2.9, Ma (2010) found that freshwater Chlorella vulgaris can grow in wastewater and yield triple the amount of biomass in a given time under mixotrophic conditions (Ma, 2016). Mixotrophic conditions include light as well as carbon sources of energy (e.g., glycerol), for algal cell growth. Ma (2016) found glycerol added to a Chlorella vulgaris culture grown in wastewater increased the microalgal biomass productivity rate (Ma, 2016). Biomass productivity with no glycerol produced biomass at a rate of $89 \mathrm{mg} / \mathrm{L}$-day, whereas when glycerol was added at $5 \mathrm{~g} / \mathrm{L}$, algal biomass productivity increased to $260 \mathrm{mg} / \mathrm{L} \cdot$ day. Carbohydrate and lipid content of algal biomass also increased with the addition of glycerol in the wastewater medium. Carbohydrate and lipid content with no glycerol produced algal biomass with $15 \%$ and $10 \%$ content respectively, whereas, carbohydrate and lipid content with glycerol produced algal biomass with $25 \%$ and $32 \%$ content respectively. 
Table 2.9: Effect on algal Chlorella vulgaris culture with the addition of glycerol (Ma, 2016)

\begin{tabular}{|l|l|l|}
\hline Algal culture properties & No Glycerol added & Glycerol maintained at 5 g/L \\
\hline Biomass growth & $0.82 \mathrm{~g} / \mathrm{L}$ & $1.82 \mathrm{~g} / \mathrm{L}$ \\
\hline Biomass productivity & $89 \mathrm{mg} / \mathrm{L} \cdot$ day & $260 \mathrm{mg} / \mathrm{L} \cdot$ day \\
\hline Carbohydrate yield & $15 \%$ & $25 \%$ \\
\hline Lipid yield & $10 \%$ & $32 \%$ \\
\hline
\end{tabular}

There are some microalgae that accumulate higher lipid composition when grown heterotrophically (Miao \& Wu, 2006). Heterotrophic microalgae, which use only organic carbon to produce energy by cellular respiration, have been shown to produce lipid content of up to $55 \%$, which is four times higher than autotrophic microalgae of the same genus (Miao \& Wu, 2006). Miao \& Wu found heterotrophic Chlorella protothecoides' lipid content reached 55.20\% when grown with $10 \mathrm{~g} / \mathrm{L}$ glucose and $0.1 \mathrm{~g} / \mathrm{L}$ glycine as part of culture medium. The carbohydrate component of Chlorella protothecoides also increased from approximately $10 \%$ to 15\%. Although some microalgae can grow heterotrophically, Christensen \& Sims (2011) indicate that using microalgae's autotrophic abilities is imperative in order for the overall process to achieve sustainability. It is likely that growing food for microalgae to consume will be more energy and GHG intensive than recycling waste nutrients and allowing light energy to support biomass growth.

Recycling glycerine, a sugar substitute, from a transesterification process back into the algae cultivation process can provide organic carbon sources that can increase biomass productivity of heterotrophic microalgae without the expense of acquiring additional sources of organic carbon (Silva et al., 2014). Recycling crude glycerol back to the cultivation stage after it has been extracted from the transesterification process not only provides algae with an additional food source but also uses some of the excess glycerol by-product.

\subsection{Microalgal Biofuel Processing Methods}

\subsubsection{Pond vs. PBR}

The most studied methods of producing microalgae for biofuels are suspended systems (Christenson \& Sims, 2011). A suspended system is one where the microalgae grows in solution 
and not on a physical structure. There are also fixed microalgal growth systems, such as algal turf scrubbers and biofilm facilities (Christenson \& Sims, 2011).

There are two main types of suspended systems used to grow microalgae: an open pond system and a photobioreactor (PBR) system. There are different configurations of each of these systems; the most promising open pond system is the raceway pond and the most promising bioreactors are flat bed and tubular photobioreactor (PBR) (Travieso et al., 2001). Table 2.10 outlines and compares the most critical system aspects of open raceway ponds and PBR microalgal growth system.

Table 2.10: Advantages and limitations of open ponds and photobioreactors adapted from (Brennan \& Owende, 2010; Kumar \& Jain, 2014; Rosello Sastre et al., 2007; Travieso et al., 2001; Vonshak \& Richmond, 1988)

\begin{tabular}{|l|l|l|}
\hline Key system aspects & Open Pond & Photobioreactor \\
\hline Light efficiency & Fairly good & Excellent (advantage) \\
\hline Temperature control & None & Fairly good (advantage) \\
\hline Gas transfer & Poor (disadvantage) & Varies \\
\hline Oxygen production & Low (advantage) & High \\
\hline Accumulation & Low (advantage) & Varies \\
\hline Hydrodynamic stress on algae & Difficult to restrict & Easier to manipulate \\
\hline Species control & Poor & Achievable (advantage) \\
\hline Sterility & Poor & High (advantage) \\
\hline Cost to scale up & $\begin{array}{l}\text { Low (lower capital costs and } \\
\text { can use non-arable land) }\end{array}$ & $\begin{array}{l}\text { High (expensive technology } \\
\text { and sometimes large land } \\
\text { space) }\end{array}$ \\
\hline Volumetric productivity & $\begin{array}{l}\text { High (larger capacity }= \\
\text { advantage) }\end{array}$ & $\begin{array}{l}\text { Low (small volume, but } \\
\text { large cross sectional area) }\end{array}$ \\
\hline Biomass productivity & Varies & Good (advantage) \\
\hline
\end{tabular}

Several studies concerning microalgal growth for biofuel production have found cultivation of microalgae in open raceway ponds (ORP) as the most cost effective method of producing microalgal biofuel (Amanor-Boadu et al., 2014; Colosi, 2012; Nanaki \& Koroneos, 2012; Ogden, 2014; Pfromm et al., 2011; Sander \& Murthy, 2010). The overall cost of fuel produced by algae grown in open ponds (US \$/ US gal) in 2014 dollars ranges from $\$ 1.65$ /gal to $\$ 25.00$ /gal (\$0.44/L - \$6.60/L) (Pienkos \& Darzins, 2009; Quinn \& Davis, 2015). The overall cost of 
fuel produced using microalgae cultivated in PBRs (US \$/ US gal) in 2014 dollars ranges from \$5.30/gal to \$33.16/gal (\$1.40/L - \$8.76/L) (Quinn \& Davis, 2015; Chisti, 2007).

Even though PBRs are more expensive to purchase and operate, they require less land than open pond systems as they can be built vertically with a resulting high surface to volume ratio (Wang et al., 2008). PBRs are superior for contamination control, increased light efficiency and increased temperature control to help maximize growth, which allows for overall increased microalgal biomass production per unit area (Shen et al., 2009). Regardless of the advantages and disadvantages of both open pond and PBRs, considering the Canadian climate and the reduced growing season, PBRs are the only option to consider if looking at continual production of microalgae in Canada (Chen et al., 2011; Singh, 2012).

Fixed microalgal growth system (i.e., algal turf scrubber and biofilm plants) studies are fewer than those supporting suspended systems. Growing microalgae using biofilms are currently expensive and there are less reliable data available as to average microalgal productivities (Barlow et al. 2016). Algal turf scrubbing operations are outdoors and even though two of the four larger microalgal growth operations in the United States use this system, considering the Canadian climate, microalgal growth using algal turn scrubbing will likely be unfeasible (Christenson \& Sims, 2011).

Flat Panel PBR and Tubular PBRs are the most promising PBRs for algal production (Singh, 2012; Wang et al., 2012). Microalgal productivity in a PBR varies with microalgal genus and specific conditions, but typical biomass productivity range is $0.05-3.8 \mathrm{~g} / \mathrm{L} \cdot$ day (Brennan \& Owende, 2010). Both $\mathrm{Hu}$ et al. (1996) and $\mathrm{Hu} \&$ Richmond (1996) have shown high culture densities using well-mixed flat panel reactors (Christenson \& Sims, 2011). However, tubular PBRs are the only type of closed system currently used at a larger scale (Chisti 2007; Mata et al. 2010). Figure 2.8 below shows an example of one of several horizontal tubular PBRs that would make up a microalgal processing facility. Wastewater can also be used to supply tubular PBRs (Willson et al., 2009). Problems that have stalled expansion of tubular PBR use however are toxic accumulation of oxygen, adverse $\mathrm{pH}$ and $\mathrm{CO}_{2}$ gradients, overheating, bio-fouling and high material and maintenance costs (Carvalho et al. 2006; Mata et al. 2010; Molina Grima et al. 1999). There is progress towards increasing both $\mathrm{CO}_{2}$ transfer and $\mathrm{O}_{2}$ release by shifting to 
bioreactors that use gas-liquid contactors, such as the rotating biological contactors (RBCs) (Patwardhan 2003; Zeevalkink et al. 1979).

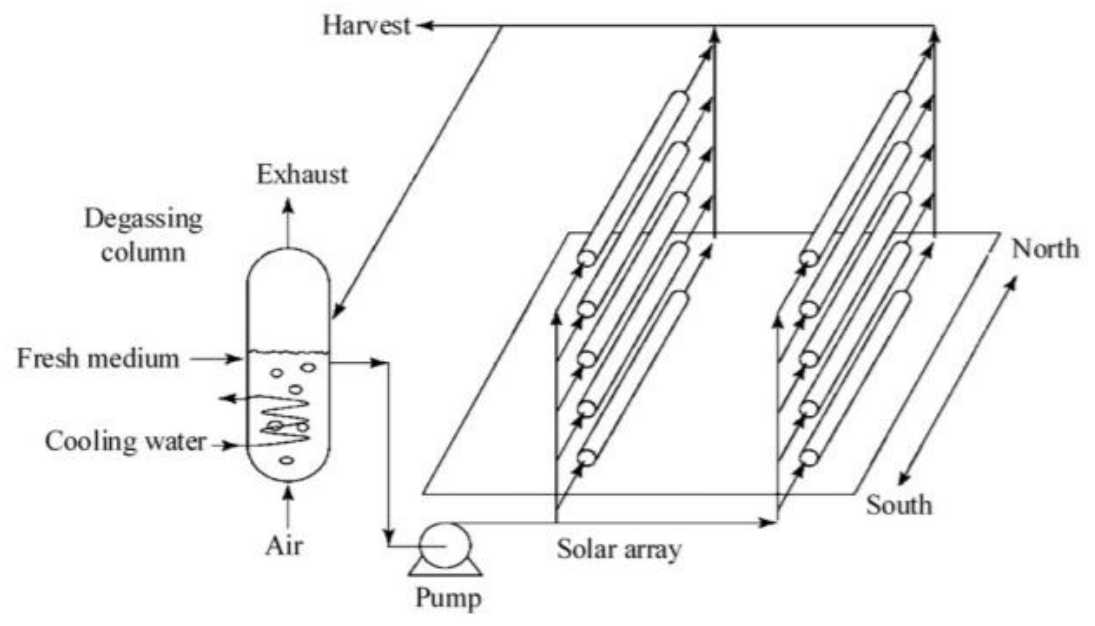

Figure 2.8: Typical horizontal tubular photobioreactor from (Harun et al., 2011)

The majority of companies using the PBR approach operate at the bench scale with some at the pilot scale (bench scale is between 10 and 1000 US gallons (of water) and pilot scale is several thousand gallons or a site of 0.5 - 10 acres) (Christenson \& Sims, 2011). Recently, pilot PBRs have been co-located at industrial facilities (e.g., cement factory) in order to recycle the carbon dioxide from flue gas (Pond Technologies Inc., 2017; Singh, 2012; Wang et al., 2012). Carbon dioxide sequestration from industry using a PBR will improve the GHG impact of both the PBR and the overall biofuel production process, assuming the microalgae is used for biofuel production. Additionally, as mentioned in section 2.3.4, the increased carbon dioxide concentrations in microalgal cultures can have positive impacts on microalgal productivity.

As mentioned in section 2.3.4, wastewater use in microalgal growth is essential for reducing the cost and the environmental impact of using fresh water resources and the use of fertilizer. Lundquist et al. 2010 indicate that operational costs would increase by $10 \%$ if non-wastewater sources were used to grow microalgae due to the loss in benefit associated with wastewater treatment and reduced fertilizer use (Lundquist et al. 2010). Pending the follow on use of microalgae, glycerol and left over biomass from the downstream microalgal production process can be recycled back in the PBR growth medium to reduce the cost of nutrients required for microalgal growth (Hazlebeck \& Dunlop 2010). Zhou et al. 2014's multilayer bioreactor system 
(20,000 L pilot scale) used centrate wastewater (i.e., supernatant from anaerobic digesters), which has relatively high levels of nitrogen and phosphorus to supplement microalgal growth in the PBR (Min et al., 2014; L. Wang et al., 2010).

There is also the option and potential of sourcing wastewater from industrial facilities (Yun et al., 1997). This study did not consider sourcing wastewater from industrial facilities because of the unknown heavy metals corresponding to certain industrial facilities and their impact on not only algal growth but also subsequent biofuel production and waste recycling.

If an onsite PBR and associated processing facility is not co-located with a large industrial facility and piping carbon dioxide from the industrial facility to a biorefinery is not an option, the industrial facility could convert its carbon dioxide to carbonate. Several microalgal species can use carbonates (i.e. $\mathrm{Na}_{2} \mathrm{CO}_{3}$ and $\mathrm{NaHCO}_{3}$ ) for cell growth (Colman and Rotatore 1995; Ginzburg 1993; Huertas et al. 2001; Merrett et al. 1996). These algae either convert the carbonate to free $\mathrm{CO}_{2}$ to facilitate use in energy production, or they directly uptake the bicarbonate. Industrial facilities can convert the carbon dioxide from stripped flue gas to carbonate salts for storage and use to grow microalgae when required.

\subsubsection{Harvesting and Pre-treatment}

Figure 2.9 depicts the schematic process for removing microalgae from the aqueous solution in which it was grown. All microalgae regardless of specific origins (e.g., freshwater lake, wastewater PBR) undergo the same process to remove growth water. Current commercial production of microalgae for pharmaceutical purposes uses a centrifuge, spray dryer and bead mill for harvesting and subsequent pre-treatment (Borowitzka, 2013). Therefore, this study outlines a similar sequential process below.

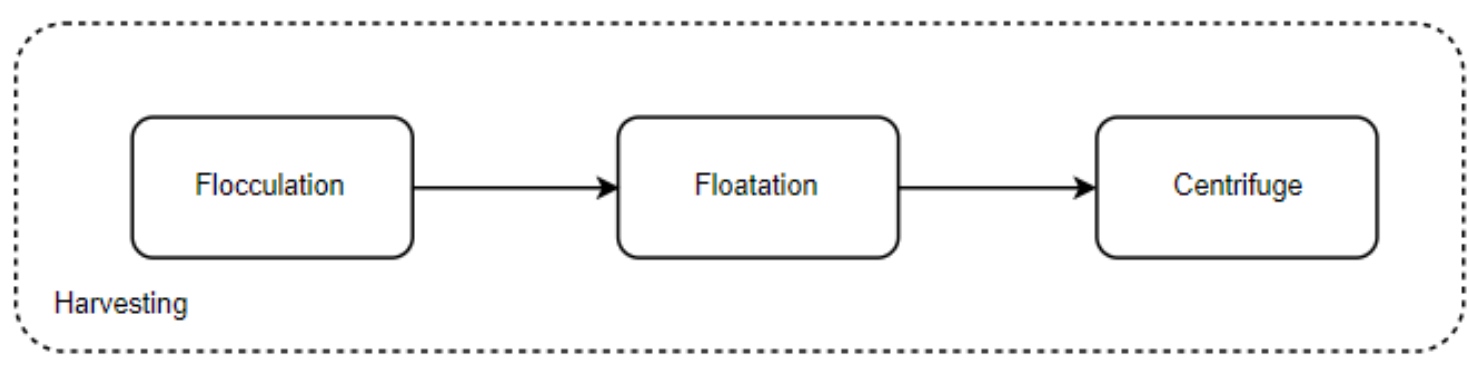

Figure 2.9: Schematic of microalgal dewatering steps 


\section{Flocculation and Filtration}

After the microalgae flows from the PBR, flocculants are added to the diluted microalgal growth solution to induce the clumping of microalgal cells into larger aggregates so these aggregates can be more easily separated from water. To induce coagulation of particles, flocculants modify cellular charges by changing culture conditions (e.g., adding an acid or a base to create $\mathrm{pH}$ changes, inducing an electric field). Flocculation, in consort with floatation, is currently the most efficient and cost effective method of initiating the extraction of microalgae from the growth water (Wang et al., 2008).

Flocculants are inorganic or organic. Inorganic flocculants include aluminum, iron or zinc sulfates or chlorides (Chatsungnoen \& Chisti, 2016). Organic flocculants include cationic polymers, starches, chitosans (i.e., linear polysaccharide), or other algae (Salim et al., 2011). Regardless of microalgal species or the residual flocculant adhering to the microalgal biomass after dewatering, organic flocculants pose little risk to the subsequent lipid extraction process (Borges et al., 2011; Chatsungnoen \& Chisti, 2016).

Inorganic sodium hydroxide $(\mathrm{NaOH})$ has proven a successful microalgal flocculant in several studies. Adjusting solution $\mathrm{pH}$ with the addition of $\mathrm{NaOH}$ effectively separated a Chlorella Vulgaris microalgae culture from solution with 98.5\% efficiency (Leite \& Hallenbeck, 2012). Yang et al. (2016) also found that high-pH induced flocculation using $\mathrm{NaOH}$ (5-7 mM) could concentrate marine Chlorella sp. strains 20-fold quickly with a flocculation efficiency of $90 \%$. Sodium hydroxide is also relatively inexpensive, non-toxic and does not affect downstream processing (i.e., water removal).

Microfiltration has also been demonstrated suitable for harvesting microalgae (Hooper et al. 1998; Hung \& Liu, 2006). However, current large-scale microalgal biomass production facilities do not use membrane filtration due to membrane fouling, pumping requirements and associated high costs (Singh et al. 2013; Wang et al. 2008). Strainers are more easily fouled due to algal cell release of extracellular organic material (EOM) resulting in interruptions of the filtration process that would not be suitable for large-scale algal biomass processing (Pittman et al. 2011). 


\section{Floatation}

Floatation uses gravity and varying densities to separate algal cultures from solution. Once the algal culture has undergone flocculation, coagulated algae settles or floats and can be removed. Solids that are hundreds of microns in size settle, whereas solids that are tens of microns in size float. Microalgae used in wastewater treatment need to be over $70 \mathrm{um}$ in size for the sedimentation method of harvesting to be successful (Brennan \& Owende, 2010). Otherwise, the sedimentation option, due to the small size of microalgae such as Chlorella, Dunaliella and Scenedesmus, takes significant time and requires a large surface area (Singh et al. 2013). Therefore, floatation technology is recommended as the best primary harvesting technique for microalgal biomass (Bunker et al., 1995; French et al., 2000; Green et al., 1996; Teixeira \& Rosa, 2007).

Suspended-air-floatation (SAF) units and Dissolved Air Floatation (DAF) units are the two main types of floatation technology used in industry today that have been successfully used to separate algal cultures (Al-Shamrani et al. 2002; Teixeira \& Rosa 2007). SAF is relatively energy efficient and requires surfactants to generate microbubbles. The reduced amount of power will likely reduce GHG impact. However, the use of surfactants will, in all likelihood, increase GHG intensity due to surfactant production as well as the additional processing required to remove the surfactant from the feedstock stream.

A DAF unit comprises of a compressor, saturator and floatation cell. To increase the air or nitrogen content of process water, the DAF compresses water in the saturator using a minimum pressure of $390 \mathrm{kPa}$ (Al-Shamrani et al. 2002). The water is released into the floatation cell where the drop in pressure causes bubbles (i.e., optimally between $10-100 \mu \mathrm{m}$ in size) to precipitate from solution in the culture medium (Al-Shamrani et al., 2002). Hydrophobic solids (e.g., algal cells) adhere to the air or nitrogen bubbles and float up to the surface of the chamber. DAF has an output of 3-6\% total solids, 99\% efficiency of biomass removal and requires energy input of approximately $0.015-20 \mathrm{kWh} / \mathrm{m}^{3}$ culture solution (Féris \& Rubio, 1999; Rance Bare et al., 1975; Shelef et al., 1984; Vandamme, 2013; Wiley et al., 2011).

The DAF unit can combine the flocculation and floatation stages (Ross et al., 2000). The unit has two chambers. In the first chamber, sodium hydroxide is added to the microalgal solution to induce microalgal flocs. The flocs flow into the floatation chamber where the microalgal flocs 
float to the surface and are collected by a skimmer. The water is continually recycled and circulated back into the process.

\section{Centrifuge}

A centrifuge generally follows after the flocculation and floatation of the microalgal cell culture. A centrifuge is timelier and more expensive than a straining filter system. However, strainers are also more easily fouled due to algal cell release of extracellular organic material (EOM) resulting in interruptions of the filtration process that would not be suitable for large scale algal biomass processing (Wang et al., 2008).

Centrifuges are usually decanters or disk stack. Decanter centrifuges require high inlet cell concentrations (>10\%), yield algal pastes of up to $40 \%$ or more in dry weight and require approximately $8 \mathrm{kWh} / \mathrm{m}^{3}$ culture volume (Shelef et al., 1984; Wiley et al., 2011). Disk Stack centrifuges operate with dilute cell concentrations ( $>0.02 \%)$, yield algal pastes of $20 \%$ dry weight and require $0.7-1.3 \mathrm{kWh} / \mathrm{m}^{3}$ culture volume (Molina Grima et al., 2003; Schenk et al., 2008). The EVODOS dynamic disk settler is $95 \%$ efficient at producing a solid yields of $25-$ $30 \%$ dry weight with relatively low energy consumption (i.e., $1 \mathrm{kWh} / \mathrm{g}$ dry wt at $0.05 \%$ feed, $0.53 \mathrm{kWh} / \mathrm{g}$ dry wt at $0.1 \%$ and $0.45 \mathrm{kWh} / \mathrm{g}$ dry wt at $0.15 \%$ feed) (EVODOS, 2011; Giang et al., 2017). As algal cell mediums leaving the floatation stage are quite dilute and disk stack centrifuges are less energy intensive, disk stack centrifuges are preferred for algal cell harvesting.

\section{Pre-treatment}

Figure 2.10 presents a schematic of the pre-treatment process. The paragraphs below explain in detail the stages in Figure 2.10. 


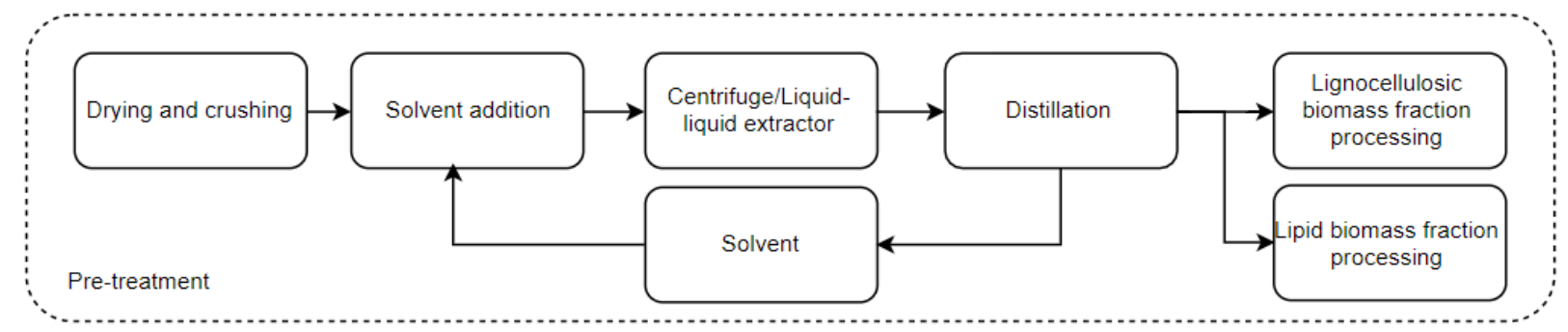

Figure 2.10: Pre-treatment process schematic for microalgal biomass - adapted from (Bradley et al. 2015; Dong et al. 2016; Gnansounou \& Raman, 2016; Pegallapati \& Frank 2016; Yuan et al., 2015)

\section{Drying and crushing}

Once the algal paste exits the centrifuge it is very perishable and must be quickly processed. Spray drying, roller drying, flash drying, vacuum drying or freeze-drying are all methods that can be used to dry the algal paste before further processing. Spray drying dries the algae in seconds. For algal paste with $80 \%$ moisture content, $30 \mathrm{kWh}$ of electric power is required to leave algal paste with 4\% water content (Petrick et al., 2013; Toptionlab, 2017). Flash drying is preferred as it allows for rapid drying with minimal power output (Petrick et al., 2013). Power requirements for flash drying is around 1.2 $\mathrm{MWh} /$ ton of water evaporated plus an additional 180 $\mathrm{kWh} / \mathrm{ton}$ of water removed for dryer operation for a total of $3.2 \mathrm{kWh} / \mathrm{kg}$ of dried algae (GEA Engineering, 2017; Petrick et al., 2013). These power requirements are based on a 30\% dry algal content entering the dryer.

Additional physical pre-treatments are used after the microalgal biomass is completely dried to help breakdown the strong bonds keeping the lignocellulosic biomass and lipids within a tightly bound fiber matrix (Lee et al., 2010; Zheng et al., 2011). Physical pre-treatment methods involve crushing the microalgal biomass with a ball mill, pulverising technology, French press or sonication technology to name a few. French presses are not appropriate for scale (Bajpai et al. 2014). As microalgal cell size can be as small as $3 \mu \mathrm{m}$ (Hu, 2014), ball milling is not appropriate (SME China Mining Equipment, 2017). Air swept pulverisers have the technology to crush very small material (Towers, 2016). Jacobson pulverisers can produce particles as low as 5 micron (Carter Day International Inc., 2012). Power requirements for the air swept 
pulveriser is approximately $90 \mathrm{HP}$ (Towers, 2016), which is similar to the pulveriser power requirements from a different manufacturer (75-100 HP) (Premium Pulman PVT Ltd., 2017).

\section{Separation}

Once the microalgal biomass has been dried and crushed, the lipids are separated from the rest of the microalgal biomass. A common way to separate lipids from the rest of the microalgal biomass is by using a mixture of methanol, ethanol, chloroform or hexane solvents (Petrick et al., 2013). These solvents disrupt the hydrogen bonds and electrostatic forces between membrane bound lipids, lignocellulosic biomass and proteins (see Figure 2.11). In Figure 2.11, both pathways (\#1-5) have the same steps (Halim et al., 2012). The solvent (i.e., polar and/or nonpolar) penetrates the cell membrane in Step 1. The solvent interacts with the lipids and forms a solvent-lipid complex in Steps 2 and 3. Finally, the solvent-lipid complex diffuses back through the cell membrane and the static solvent film to settle in the bulk solvent in Steps 4 and 5. Hydrogen bonds strongly bind some of the lipids to proteins in the cell; thus, the complete extraction of lipids requires both polar and non-polar solvents to break these bonds (Halim et al., 2012). The solvents ideally should have a low boiling point, be non-toxic and easily recoverable, as these chemicals should be continually recycled (Chatsungnoen \& Chisti, 2016).

The Bligh-Dyer method is the most common process used to extract lipids from solution (Bai et al., 2014; Bligh \& Dyer, 1959). Chloroform:methanol in a 1:1 (v/v) or 1:2 (v/v) mixture is added to the microalgal biomass (Bai et al., 2014). Water can be added to the algal biomass solvent mixture and the solution separates into methanol and chloroform layers (Bai et al., 2014). Carbohydrates and proteins are soluble in methanol and lipids are soluble in chloroform (Bligh \& Dyer, 1959). A liquid-liquid decanter or a centrifuge separates the two streams and distillation separates the corresponding solvents.

As chloroform is environmentally toxic (Petrick et al., 2013), other methods of separation are being considered for this study. Hexane extraction requires the addition of hexane to the microalgal biomass with the subsequent use of a centrifuge and distillation column to assist phase separation and subsequent lipid fraction separation (Dong et al., 2016). Using only hexane as the separation solvent, however, has proven to extract just over $20 \%$ of the available lipids (Petrick et al., 2013). Although these results are incongruent with those studies using hexane to 
extract lipids performed by Bai et al (2014) and Dong et al. (2016). Bai et al. (2014) recovered over $90 \%$ of the available lipids and Dong et al. (2016) recovered over $87 \%$ of the available lipids. Both Bai et al. (2014) and Dong et al. (2016) used an acid pre-treatment prior to lipid extraction.

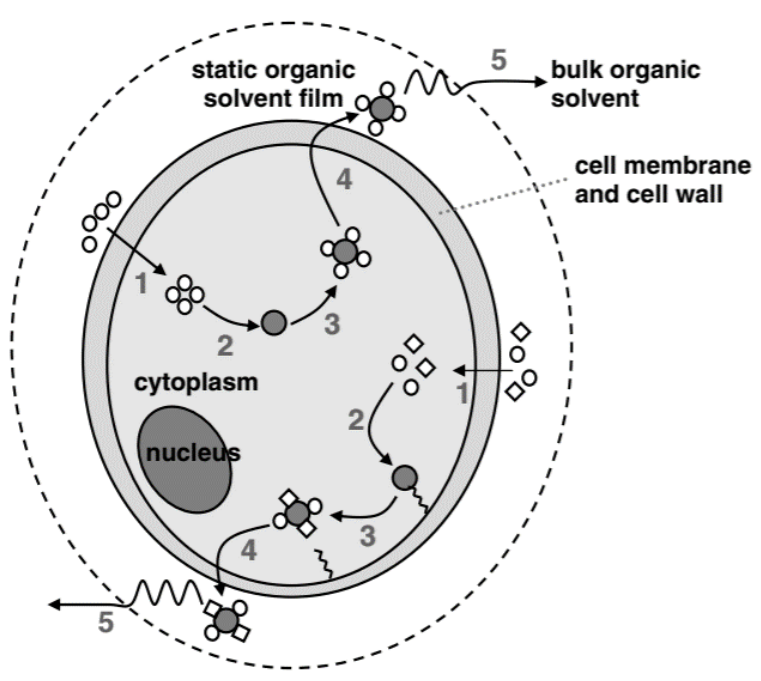

Figure 2.11: Scematic of solvent lipid-extraction mechanism for microalgal cells. Top pathway (1-5) is the mechanism for non-polar solvent (e.g., hexane) extraction. Bottom pathway (1-5) is the mechanism for the non-polar/polar solvent (e.g., hexane \& ethanol) mixture extraction. Lipids (dark circles), Non-polar solvent (white circles), polar solvent (diamonds), adapted from (Halim et al., 2012)

Additional processing of both lignocellulosic and lipid fractions

The lignocellulosic biomass fraction of the microalgal biomass is subject to additional physical and/or chemical pre-treatment to prepare the microalgal biomass for conversion to fuels. This additional pre-treatment is required to further breakdown strong bonds keeping the lignocellulosic biomass within a tightly bound fiber matrix (Syed, 2012). Physical and chemical pre-treatment techniques include steam explosion, acid wash and others outlined in Harmsen et al. (2010). A combination of the two types of pre-treatment have the potential to produce better overall yields of ABE but is not always necessary (Harmsen et al. 2010).

Additional Lignocellulosic biomass pre-treatment includes an acid pre-treatment, steam treatment or heat treatment and subsequent acid neutralization. Steam treating, also known as 
steam explosion, is used most often in industrial processes due to high yield. In this study's case, the appeal is the lack of additional chemical required. Steam explosion usually takes place at temperatures ranging between 160 and $260^{\circ} \mathrm{C}$ and at pressures ranging between 0.69 and 4.83 MPa (Taherzadeh \& Karimi, 2007). Adding a catalyst decreases the time and temperature needed overall thereby decreasing the likelihood of inhibitor production during the treatment (Kumar et al., 2009). After the catalyst, typically a strong acid, is added and allowed to react, the biomass is then moved to a steam reactor, which operates at approximately $190^{\circ} \mathrm{C}$ and 1.3 $\mathrm{MPa}$ (Begum \& Dahman, 2015). Once the biomass is heated and pressured, it is moved to a second, larger reactor where the biomass experiences a drastic drop in pressure that will cause it to explode. A strong base then neutralizes the treated lignocellulosic material to increase the $\mathrm{pH}$. Additional chemical pre-treatment of the lipid fraction could be used if the lipid stream has a larger amount of FFA. As mentioned in section 2.3.2, a lipid stream with large amounts of FFA is not suitable for BD production. Treating the lipid stream with dilute acid can reduce the FFA content and increase FAME yield during the transesterification process (Bai et al., 2014; Dong et al., 2016). Also, completing glycerolysis can reduce FFA and increase biodiesel yield during transesterification (Silva et al., 2014). Glycerolysis uses the waste glycerol from the transesterification process and through reactions with water/supercritical carbon dioxide (i.e., at high pressure) or with a base catalyst, produces more Triglycerides (TG), diglycerides (DG) and monoglycerides (MG) suitable for conversion to FAME (Silva et al., 2014).

Wet pre-treatment and extraction is a process that has the potential to be more sustainable. This wet treatment process would save on drying energy requirements. Dong et al. (2016) studied processing microalgae biomass using a wet pre-treatment process similar to that used in the corn ethanol industry (Dong et al. 2016; Noureddini et al. 2009). Dong et al. 2016 used a centrifuge to increase solid content of microalgal slurry to $20 \mathrm{wt} \%$, pre-treated the biomass with dilute acid, fermented the entire slurry to extract ethanol (i.e., 79\% sugar extracted) and then transesterified the rest of the biomass (i.e., $87 \%$ oil converted to FAME) to produce BD. The left over biomass was then assumed to be used for power and heat production. The total fuel energy yield was 126 GGE (gallons of gasoline equivalent) per ton of biomass. Dong et al. 2016 found that most of the total process cost was still associated with cultivation and harvesting of the microalgae, which includes the wet extraction process. Unfortunately, Dong et al. 2016 did not specify how 
much the wet extraction process contributed to aforementioned cost, as the paper's focus was on yields rather than the breakdown of energy requirements.

\subsubsection{ABE production from lignocellulosic biomass}

The ABE production process consists of hydrolysis, fermentation and subsequent distillation to produce bio-acetone, bio-butanol and bio-ethanol. Besides alcohol products, fermentation of lignocellulosic biomass also produces unreacted lignocellulosic biomass, carbon dioxide and heat (Arbor, 1986). The unreacted lignocellulosic biomass can be co-digested with other process organic wastes, the carbon dioxide can be used for microalgal growth and the heat can be used for process heating requirements.

\section{Hydrolysis}

Hydrolysis is required to convert cellulose and hemi-cellulose to fermentable products. Hydrolysis, in this case, can follow acid hydrolysis or enzymatic hydrolysis methods. Acid hydrolysis adds either dilute or concentrated acid to the algal culture to produce glucose molecules and other short polysaccharide chains (Maurice 2011). The disadvantage of the dilute acid method is a relatively low yield of glucose when compared to the concentrated acid method and enzymatic hydrolysis method. The disadvantage of the concentrated acid method, even though it produces more glucose than the dilute acid method, is that the concentrated acid method requires an additional step to recover it from solution, as it would kill the yeast required for the following fermentation step (Maurice 2011).

Enzymatic hydrolysis uses enzymes to cleave bonds between molecules with the help of water. This process occurs in several stages using several different enzymes. First, enzymes digest the lignin producing cellulose. Then, the enzymes endocellulase and exocellulase digest the cellulose to produce polysaccharides. Another enzyme, beta-glucosidase, is subsequently introduced to convert the polysaccharides to glucose (Maurice, 2011). The ideal pH and temperature for hydrolysis is around 5 and $50^{\circ} \mathrm{C}$ respectively. One disadvantage of enzymatic hydrolysis is the rate of hydrolysis decreases as the concentration of the desired product, glucose, 
increases. Excess glucose inhibits the enzymes ability to convert cellulose to glucose. Another disadvantage is a given batch takes several days to convert to glucose and is relatively expensive compared to the acid hydrolysis method (Taherzadeh \& Karimi, 2007).

\section{Fermentation}

Fermentation requires a microorganism (e.g. yeast or bacteria) to convert hydrolyzed glucose into alcohols. ABE fermentation occurs in two main stages. The first stage, acidogenesis, produces butyric acid and acetic acid. The second stage, solventogenesis, produces the final products acetone, butanol and ethanol (Kótai et al., 2013).

There are several fermentation strategies currently used and under study to produce $\mathrm{ABE}$ from lignocellulosic biomass. The two most common processes are separate enzymatic hydrolysis and fermentation (SHF) and simultaneous saccharification and fermentation (SSF) (Taherzadeh \& Karimi, 2007).

SHF treats the lignocellulosic algal biomass to hydrolysis and then fermentation separately in two different vessels. The advantage here is that the optimal temperature and culture conditions can be achieved for each stage of the SHF process. Cellulose hydrolysis optimal temperature range is 45 to $50^{\circ} \mathrm{C}$ whereas fermentation prefers a temperature range between 30 and $37^{\circ} \mathrm{C}$ (Taherzadeh \& Karimi, 2007). A disadvantage of SHF is the released sugar from the hydrolysis reaction can inhibit this same reaction. With as low a concentration of $3 \mathrm{~g} / \mathrm{L}$, glucose can reduce beta-glucosidase activity by $75 \%$ (Taherzadeh \& Karimi, 2007). Another disadvantage of SHF is the chance of batch contamination over the one to four day process.

To overcome some of the disadvantages of SHF mentioned above, SSF combines the hydrolysis and fermentation processes. As enzymatic hydrolysis produces glucose, the fermentation yeast or bacteria subsequently consume it (Procentese et al., 2014). This assembly line like process prevents inhibition effects of glucose and cellobios found in SHF. Furthermore, since the SSF process occurs in only one vessel, the risk of contamination is reduced. A disadvantage of SSF is butanol inhibition. Butanol at high concentrations can become toxic for microorganisms. Another drawback to SSF is that the single vessel temperature cannot be adjusted for both hydrolysis and fermentation optimal temperatures. A temperature of $38^{\circ} \mathrm{C}$ is normally the 
standard temperature for hydrolysis. However, there are some bacteria, such as Kluyveromyces marxianus and fused strains of Clostridium beijerinckii and Clostridium thermocellum, that have higher optimal temperature ranges and can operate at temperatures close to the hydrolysis temperature range (Saini et al. 2015).

SHF or SSF processes use either yeast or bacteria. Bacteria tend to have stricter reaction condition requirements than yeast. For example, purging the reactor of oxygen with nitrogen and creating an anaerobic environment is optimal when using bacteria for the fermentation step, as oxygen is an inhibitor of fermentation reactions (Maurice et al. 2011; Stanbury et al. 2016). Contamination is also of real concern for bacteria, whereas yeast does not require high sanitation standards since it has such a fast growth rate. Another advantage of using yeast is that it can be purchased and used without alteration whereas bacteria need to be inoculated and scaled up before use (Pfromm et al., 2011). However, yeast tends to operate at lower than optimal temperatures for ABE fermentation making SSF a less desirable option when using yeast. Moreover, using yeast for the fermentation process requires the growth and addition of enzymes, whereas bacteria create their own required enzymes. Therefore, even though bacteria are more sensitive, they are the better choice for SSF.

In recent years, new studies have modified $\mathrm{ABE}$ production by genetically engineering Clostridium acetobutylicum and Clostridium beijerinckii bacteria strains to improve the fermentation process (Castro et al., 2015; Ellis et al., 2012; Huesemann et al., 2012; Syed, 2012; Yen \& Wang, 2013). These modifications have improved ABE yields, and specifically biobutanol yields. A study conducted by Begum \& Dahman (2015) found a fused Clostridium beijerinckii with Chlostridium thermocellum $(\mathrm{CbCt})$ achieved a 10\% higher yield of butanol and a $26 \%$ higher yield of $\mathrm{ABE}$ (i.e. gram $\mathrm{ABE} /$ gram sugar) than the reigning study (Begum \& Dahman, 2015). Even though the feedstock used to produce ABE was wheat straw, it can be assumed that cellulose and hemicellulose from microalgal biomass has the potential to produce similar results (Ellis et al., 2012; Qureshi et al., 2006).

There are several different types of reactors used for fermentation. These include continuous stirred tank reactors (CSTR), bubble column reactors, airlift reactors, and fluidized bed reactors (Spier et al., 2011). The preferred fermentation vessel for sensitive organisms is an airlift bioreactor. An airlift bioreactor uses nitrogen gas for mixing and not a more invasive 
mechanical stirrer used in a CSTR (Spier et al., 2011). An airlift reactor has gas emerge and rise from all points along a tube running through the centre of the reactor. This design makes for effective mixing.

\subsubsection{Biodiesel production from lipids}

Microalgal lipids have a high viscosity and are thus incompatible for direct use in a CIE (Silva et al., 2014). Therefore, as indicated in section 2.3.2, the BD production process converts lipids to FAMEs and FAMEs are compatible for use in a CIE. Triglycerides (TG) and TG derivatives (i.e., monoglyceride (MG) and diglyceride (DG)) are the easiest lipids to convert to FAMEs. If the microalgal oil has a high acid number (i.e., high FFA content), the pre-treatment of FFAs with dilute acid allows the additional conversion of FFA to FAMEs during the subsequent transesterification process (Dong et al., 2016). Section 2.4.2 outline the dilute acid treatment and glycerolysis processes. TG, DG and MG are converted to FAME and glycerol (GLY) by the overall transesterification reaction (1) through the stepwise reactions (2) through (4) (Silva et al., 2014)

Overall reaction:

$$
1 T G+3 M e O H \leftrightharpoons 1 G L Y+3 F A M E
$$

Stepwise reaction:

$$
\begin{aligned}
& T G+M e O H \leftrightharpoons D G+F A M E \\
& D G+M e O H \leftrightharpoons M G+F A M E \\
& M G+M e O H \leftrightharpoons G L Y+F A M E
\end{aligned}
$$

One mole of FAME is generated per mole of methanol $(\mathrm{MeOH})$ reacted in each of the above reactions (2-4), which produces three moles of FAME (Ataya et al. 2007). Although methanol can be obtained from renewable sources for this reaction, it is usually derived from natural gas, a non-renewable resource (Knothe, 2010). 
Either an acid or base catalyst is typically used to facilitate the transesterification process (Ahmad et al. 2013; Kumar and Jain 2014; Xu et al. 2006). Types of catalysis include sulfuric acid, hydrogen chloride and sodium hydroxide (Kumar \& Jain, 2014). Table 1 in Liu et al. (2011) includes a list of common catalysts (Liu et al., 2011).

Industrial operations with commercial biodiesel production employs homogeneous alkali catalysts as they produce higher yields and faster reaction rates (Alsalme, 2008; Silva et al., 2014). These alkali catalysts need to be washed out of the FAME and glycerol product phases in order to be recirculated (Silva et al., 2014). There is also the option of using a solid base catalyst, thus circumventing the washing requirements (Liu et al., 2011; Silva et al., 2014). Ahmad et al. (2013) found a 92\% BD yield from oil extracted from a mixed microalgal culture grown in wastewater using the solid base catalyst, sodium methoxide. The sodium methoxide was separated out of the $\mathrm{BD} / \mathrm{glycerol}$ phase using a separating funnel under vacuum (Ahmad et al., 2013).

The main reason for lower rates of transesterification reactions using acid catalysts is the mass transfer limitations; the acid and alcohol form immiscible phases in the system (Kelkar et al., 2007). Regardless, transesterification uses acid catalysts instead of alkaline catalysts to allow for varied feedstock. If the BD production process uses mixed strain microalgae and the oil has an FFA content of larger than $0.5 \%$, an alkaline catalyst would produce large amounts of soap formation. This soap formation would make the removal of the BD from the glycerol more difficult, thus resulting in reduced overall BD yield (Guan et al., 2009). The water concentration of the microalgal lipid solution should be limited to less than $0.1 \%$ water as water too leads to soap formation when using an alkaline catalyst (Guan et al., 2009). Using an acid catalyst, such as hydrosulfuric acid, requires long transesterification reaction times (i.e., more than 1 day) therefore, heat and high concentrations of methanol are used to speed up the process (Guan et al., 2009; Kumar \& Jain, 2014). It is these difficulties and drawbacks that are the main impediments of producing renewable fuel from microalgal oils (Kumar \& Jain, 2014).

To avoid the use of catalysts all together, the transesterification process can proceed using supercritical conditions. Super critical $\mathrm{CO}_{2}$ is the most popular supercritical fluid because of its low critical parameters and ready availability (Bernal et al., 2012). However if supercritical methanol is used in the transesterification process, it acts as not only a catalyst and solvent, but 
also a needed reactant for the process (Kusdiana \& Saka, 2004). The critical parameters for methanol are $239.6^{\circ} \mathrm{C}$ and $8.09 \mathrm{MPa}$, therefore, the transesterification process must be at least this temperature and pressure (Bernal et al., 2012).

Another advantage of the non-catalytic supercritical methanol transesterification process is the output of highly purified extracts (i.e., FAME and glycerol) (Patil et al., 2012). This process eliminates a neutralization step as no catalyst is used and FFAs are converted to FAMEs along with TG and TG derivatives, which increases the overall BD yield (Kusdiana \& Saka, 2004). Unlike the subcritical traditional transesterification process, water does not affect the supercritical process (Kusdiana \& Saka, 2004). Conversion of oil to FAME is rapid (i.e., less than 3 minutes) with a $98 \%$ conversion at $10-20 \mathrm{MPa}$, between 375 and $400^{\circ} \mathrm{C}$ with a methanol:oil ratio of 3:1 to 6:1 (Bernal et al. 2012; Marulanda et al. 2010; Pinnarat and Savage 2008). Drawbacks of this process are high operational energy consumption, high equipment cost and safety concerns associated with operations at supercritical conditions (Bernal et al., 2012) Ultrasonic and hydrodynamic cavitation can also facilitate the transesterification process (Kumar \& Jain, 2014). Ultrasonic power used for enhancing transesterification has low maintenance costs, reduces process time and is functionally an environmentally friendly technology (Mason \& Lorimer, 2002; Suslick, 1989). Ultrasonic heating produces cavitation in the reactor resulting in emulsion droplets forming between the alcohol and oil phases (Mostafaei et al.,2015). Naderloo et al. (2017) used low frequency ultrasonic power (28 and $40 \mathrm{kHz}$ ) to convert vegetable oil TG, FA and FFA into BD using methanol in the presence of each of the three catalysts in separate trials: $\mathrm{NaOH}, \mathrm{H}_{2} \mathrm{SO}_{4}$ or $\mathrm{KOH}$ (Naderloo et al. 2017). Naderloo et al. (2017) found that with significantly less ultrasound than in previous studies, the amount of catalyst could be reduced, the amount of methanol could be reduced, and saponification could be virtually eliminated with a final oil conversion of between 95 and 97\%. Experiments required 6:1 molar ratio of methanol to oil, a catalyst quantity of $1 \%$ by weight, $45^{\circ} \mathrm{C}$ temperature and 3 minute reaction time. Overall energy input required was 36.652 MJ/L of BD produced and output energy was 47.005 MJ/L of BD produced. This energy ratio resulted in an ERI of 1.283 (Naderloo et al., 2017).

Transesterification with hydrodynamic cavitation carried out by Kelkar et al. (2007) with methanol used a molar ratio of FA to alcohol of 1:10). The process requires excess methanol since the water formed during the esterification reaction dissolves in this excess methanol 
(Kelkar et al., 2007). The process also requires a catalyst of $1 \%$ (w/w) of $\mathrm{H}_{2} \mathrm{SO}_{4}$. Ambient operating conditions of $28^{\circ} \mathrm{C}, 1$ atm pressure and reaction time of 3 hours was able to produce an over $90 \%$ conversion $(\mathrm{mol} \%)$. Superacid clay slowed down the reaction time and the heterogeneous catalyst could not be recycled in this case, which is a drawback. Regardless, cavitation in combination with acid and methanol is an excellent way to achieve process intensification of biodiesel synthesis (Kelkar et al., 2007). The power usage for this process was a $1.5 \mathrm{~kW}$ multistage centrifugal pump used for cavitation purposes. Hydrodynamic cavitation proved a more energy efficient way of creating turbulence that will make acid and alcohol more miscible when compared to traditional transesterification without cavitation (Kelkar et al., 2007).

CSTRs are the most common reactor used in industry today and are suitable for the transesterification process (Fogler, 2006). CSTRs can be modified to operate under supercritical conditions as required. CSTR operate at steady state (i.e., same temperature, pressure and reaction rate throughout). The reactor receives a continuous stream of reactants and outputs a continuous stream of products and by-products. Cleaning the reactor is relatively easy and its operation inexpensive, when compared to other reactors (i.e., batch reactors). CSTRs do not need to be opened on a regular basis; interrupting production is only required twice a year for maintenance (Fogler, 2006).

\subsubsection{Recovery of products}

Distillation, adsorption, gas stripping, liquid-liquid extraction, pervaporation or evaporation methods will recover bio-acetone, bio-butanol and bio-ethanol. The most widely used practice in the fuel refining industry for recovery of fuel products is distillation (Kujawska et al., 2015). Separation by distillation occurs due to differences in material volatilities. Long tube vertical evaporators in industry today are the most efficient units available to separate solutions by distillation. Long tube vertical evaporators, specifically the falling-film type, use gravity to help the solution form a thin film moving down the inner wall of the tube. Gravity speeds up the process reducing the amount of time the solution spends in the tube and yielding higher heattransfer coefficients. The higher the heat transfer coefficient $\left(\mathrm{W} / \mathrm{m}^{2} \cdot \mathrm{K}\right)$, the faster the heat transfer, and the faster, in this case, the solvent evaporates. Distillation is favourable because of 
its high recovery rate, multi-stage operation and ease of scale up. A disadvantage of distillation is the energy required for this process when dealing with low alcohol concentrations.

Condensers are required to convert the vaporized fuels back into liquids for transport and distribution. Shell and tube heat exchangers often use water as the cooling fluid. These heat exchangers do require regular maintenance to prevent tube fouling and can be quite large, but their versatility (e.g., installation positions and use) and low operating costs outweigh these disadvantages.

After the transesterification process, as much of the extra methanol as possible is evaporated, condensed and recycled. This process uses similar equipment mentioned in the paragraphs above. Recovery of BD (FAME) and residual oil from glycerol use a centrifuge or liquid-liquid decanter. The density of glycerol and FAME is approximately $1,261 \mathrm{~kg} / \mathrm{m}^{3}$ and $874 \mathrm{~kg} / \mathrm{m}^{3}$ respectively. Due to the differences in density, the top of the decanter draws the light FAME and the bottom of the decanter collects the denser glycerol and adhered residual methanol not previously evaporated. Although using a decanter takes more time per volume of solution than a centrifuge, it is simple, operates continuously and a lot less expensive than a centrifuge (Ahmad et al., 2013). FAMEs can then be evaporated to separate them from the residual lipid stream and then condensed for subsequent refining, transport and distribution.

\subsubsection{Refining Biodiesel and ABE}

After evaporating and condensing the FAME product to remove the FAME from the residual lipids, there may be additional refining or processing required to ensure the BD is at standard for use. Additional washing with petroleum ether and water as well as subsequent evaporation may be required (Kumar \& Jain, 2014). This washing would require additional materials and energy. It is likely that these additional processes would be completed at a different facility (i.e., a petroleum refining facility) as this facility would likely already be the hub for subsequent fuel transport and distribution (Graham, 2011).

Neat or $100 \%$ ethanol (or butanol) is usually prepared by azeotropic distillation (Wittcoff et al., 2004). The mixture requires benzene for the distillation process. The ternary azeotrope that 
distills takes the benzene and water with it, leaving anhydrous alcohol as the bottom product. Another method of alcohol purification uses countercurrent extraction with glycerol or ethylene glycol. The added component (i.e., glycerol or ethylene glycol) hydrogen bonds to the water and allows anhydrous alcohol to be drawn from the top of the processing column (Wittcoff et al., 2004). Similar to follow-on FAME processing, a petrochemical facility will likely complete the alcohol refining for use as fuel for the same reasons as above.

\subsubsection{Anaerobic digestion and power generation}

\section{Digestion}

Figure 2.12 depicts the part of the wastewater treatment process that involves the anaerobic digestion process associated with nutrient recycling and power generation.

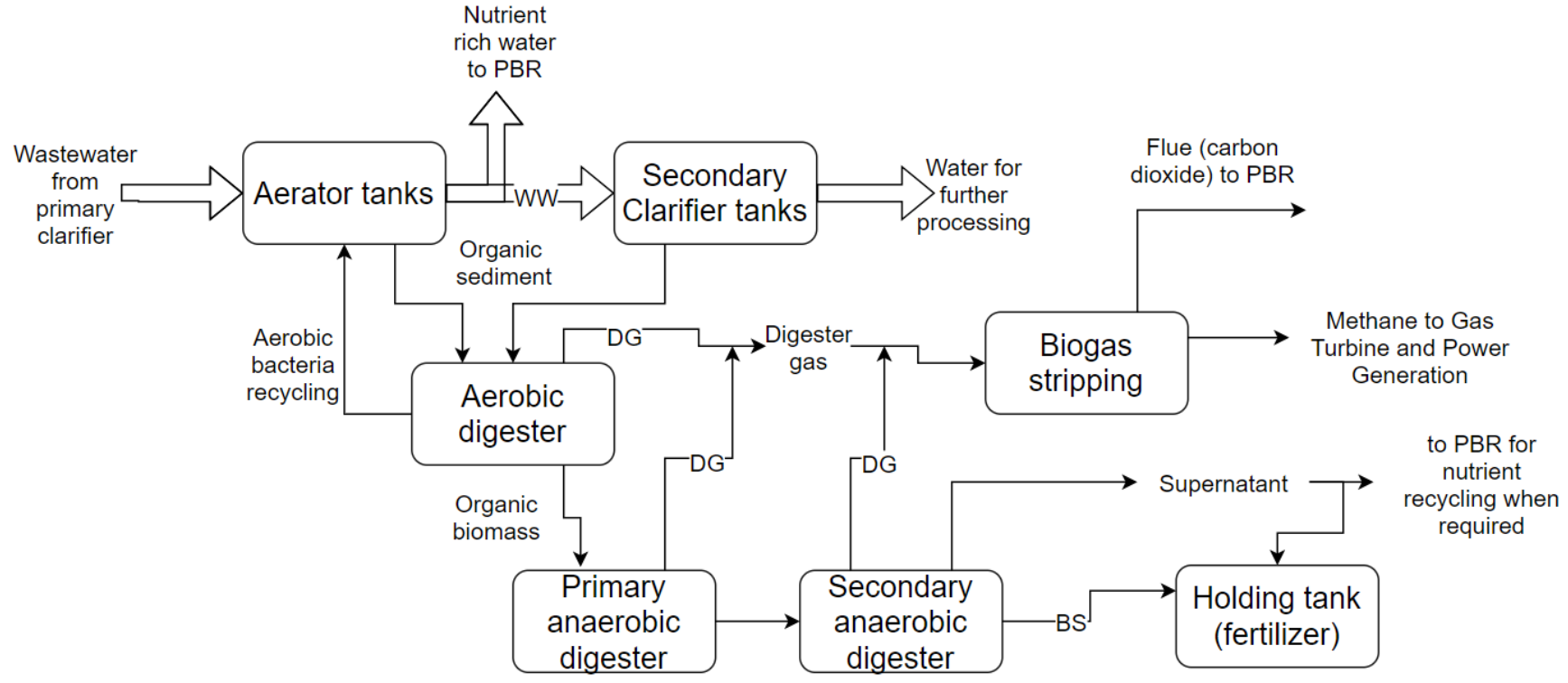

Figure 2.12: Wastewater treatment process flow as it concerns microalgal biomass growth, nutrient recycling and power generation, WW - wastewater, DG - digester gas, BS - biosolids, modified from (Tchobanoglous et al. 1991; Yun et al. 1997)

Anaerobic digestion is the biological conversion of organics to biosolids and biogas (i.e., referred to as digester gas in Figure 2.12) in an oxygen free environment (Parkin \& Owen, 1986). Both biosolids and biogas are important products for use in the microalgal biomass production 
process. The supernatant from the secondary anaerobic digester contains nitrogen and phosphorus from the microalgal biomass and can be recycled continuously for microalgal biomass growth as required (Kim et al. 2015). The biosolid portion can be used as fertilizer for farms in the local area (Gómez et al. 2006). Biogas components (i.e., carbon dioxide and methane) help supplement carbon in the PBR for microalgal growth as well as on-site power generation.

To maintain the fermentation process and subsequent biogas and biosolid formation in the anaerobic digesters, it is important to maintain a stable population of methane forming bacteria (Parkin \& Owen, 1986). To do this, optimizing digester temperature, adequate mixing, long enough retention time, adequate nutrients and the absence of toxic materials is required.

In order to maintain temperature and mixing requirements, digesters require thermal and electrical energy (Frank et al. 2011). Approximately 30\% of the heat value of burning biogas is sufficient to provide enough heat to maintain digester temperature (Collet et al., 2011). Therefore, it can be assumed that when biogas is burnt for power generation purposes, this heat generated will be sufficient to maintain a $35^{\circ} \mathrm{C}$ digester temperature. Electrical power requirements for mixing are $0.11 \mathrm{kWh} / \mathrm{kg}$-TS (Collet et al., 2011). Total solids (TS) is the solid organic content in the digester.

Another key factor to maintaining a stable population of methane forming bacteria is an adequate retention time in the digester to allow substrate metabolism and to prevent washout of bacteria (Barrie wastewater treatment facility, 2017; Parkin \& Owen, 1986). Minimum adequate time is 10 to 15 days in primary anaerobic digester and 15 days in the secondary anaerobic digester (Barrie wastewater treatment facility, 2017). Thus, SRT is a very important and often limiting factor concerning bacterial culture maintenance (Parkin \& Owen, 1986).

Maintaining nutrient balance within the digester is also important to maintain bacteria culture. Considering protein will dominate inputs to the digesters if all wastewater is maximized for microalgal growth, there will be a need to maintain optimal nutrient levels for the bacteria (Olsson et al., 2014). This might require more than glycerol inputs from the transesterification output. 
Biogas from the digestion process consists primarily of methane (50-70\%) and carbon dioxide (30-50\%) and also trace amounts of dihydrogen sulfide, water vapor, ammonia and siloxanes (Parkin \& Owen, 1986; Shen et al. 2017). The digester produces a certain amount of methane and carbon dioxide depending on the final amount of lignocellulosic biomass, proteins and lipids that enter the digester. An added advantage of digesting a multitude of organic material, wastewater organic material and microalgae, is the increase in carbon dioxide yield and biological methane potential (BMP) (Ağdağ \& Sponza, 2007; Jingura \& Matengaifa, 2009; Olsson et al., 2014). Table 2.11 contains the methane yield for lignocellulosic biomass, proteins and lipids.

Both methane and carbon dioxide are valuable components of the biogas. The carbon dioxide can be recycled back to the PBR as a carbon nutrient supplement and the methane can be substituted for NG for power generation (Wiley et al., 2011). For this to occur, the biogas is stripped to separate the carbon dioxide and methane (Kapdi et al. 2005). The stripping energy requirements range from 0.15 to $0.5 \mathrm{kWh} / \mathrm{N} \cdot \mathrm{m}^{3}$ (N refers to the temperature measurement at STP) (Bauer et al. 2013). The methane burnt in a gas turbine to turn a generator for on-site power production as part of a power generation system of less than $5 \mathrm{MW}$ had a power generation conversion efficiency of 30\% (Cengel \& Boles, 2002; Frank et al., 2011).

Table 2.11: Biomass methane yield from anaerobic digestion (Frigon \& Guiot, 2010)

\begin{tabular}{|l|l|}
\hline Biomass & Methane yield \\
\hline Carbohydrates (lignocellulosic biomass) & $0.37 \mathrm{~m}^{3} / \mathrm{kg}$ \\
\hline Proteins & $0.51 \mathrm{~m}^{3} / \mathrm{kg}$ \\
\hline Fats (lipids) & $1.0 \mathrm{~m}^{3} / \mathrm{kg}$ \\
\hline Plant biomass & $0.48 \mathrm{~m}^{3} / \mathrm{kg}$ \\
\hline
\end{tabular}

Observed trends in biogas usage in urban areas larger than 150k in the US and 50k in Canada found that $66 \%$ of facilities had an anaerobic digester system and of those only $35 \%$ had an energy recovery system (Lackey et al. 2015). This means that some wastewater treatment plants do not have the facilities to recycle nutrients for PBR growth nor do some have the ability to reduce their power consumption with an on-site power generation system. Ideally, a process, 
such as the one in this study, would require a biofuel processing facility co-located with a wastewater treatment plant with digesters and co-generation.

\subsubsection{Green Process Engineering}

The term "Green Process Engineering" or "Green Engineering" emerged in the last two decades in education and industry circles because of growing environmental concern with traditional manufacturing and processing of materials (García-Serna et al., 2007; Poux, 2014a, 2014b; Toulouse INP (Universite), 2018). Green Process Engineering (GPE) is defined as the design, commercialization and use of feasible and economical processes and products while minimizing a) the generation of pollution at the source and b) the risk to human health and the environment (Patel et al., 2014).

Inroads have been made in education and industry concerning the expansion and understanding of GPE (Toulouse INP (Universite), 2018; University of Western Ontario, 2018). International working groups, such as the Toulouse INP conference, have and continue to focus on introducing and discussing GPE concepts. The University of Western Ontario has created a GPE based chemical engineering degree and Environmental Engineering is offered in several Canadian universities and colleges (Kimantas, 2014; University of Western Ontario, 2018). The emphasis in GPE in industry, however, as per the definition, continues to focus on economic feasibility. This hierarchy leaves closed loop systems and environmental risk as secondary priorities or primary priorities only if they reduce cost.

Naturally, the economic feasibility of a new green engineering project is an undeniable requirement given the current economic system. However, in addition to a cost analysis, including proof of a green engineering process could provide a company with grant capital that could shift the balance between start up project economic unfeasibility and feasibility. For example, as explained in section 2.2.2, the Ontario government's CleanTech strategy supports companies developing green engineering processes (The Government of Ontario, 2018). With this new strategy, the Ontario government is not only looking to streamline the environmental compliance process and provide opportunities to acquire capital for green energy companies, which has been the focus for several years, but for bio-based companies in general (The Government of Ontario, 2018). Once a project completes its overall cost-analysis, there is 
already an understanding of material requirements and sources. At this point, completing a preliminary LCA would be straightforward and would provide good proof of "green engineering" for CleanTech associated grant applications. Furthermore, completing a preliminary LCA would serve to shed light on environmental invasive and energy intensive impact areas, which could foster design changes prior to project planning phases that offer reduced flexibility in overall concept (Hauschild et al., 2004).

2.5 Life Cycle Assessments of microalgal biodiesel

A life cycle assessment is a method to assess the life cycle impact of a particular product or process (International Organization for Standardization, 2006a). The life cycle of a product, for example, consists of all the stages the product experiences and influences throughout its lifetime. In Figure 2.13, the technosphere depicts how anthropogenic processes draw material from the natural environment in order to produce a product and then the technosphere is required to put the material back into the natural environment when the product reaches the end of its anthropogenic usefulness (Thinkstep GaBi, 2017).

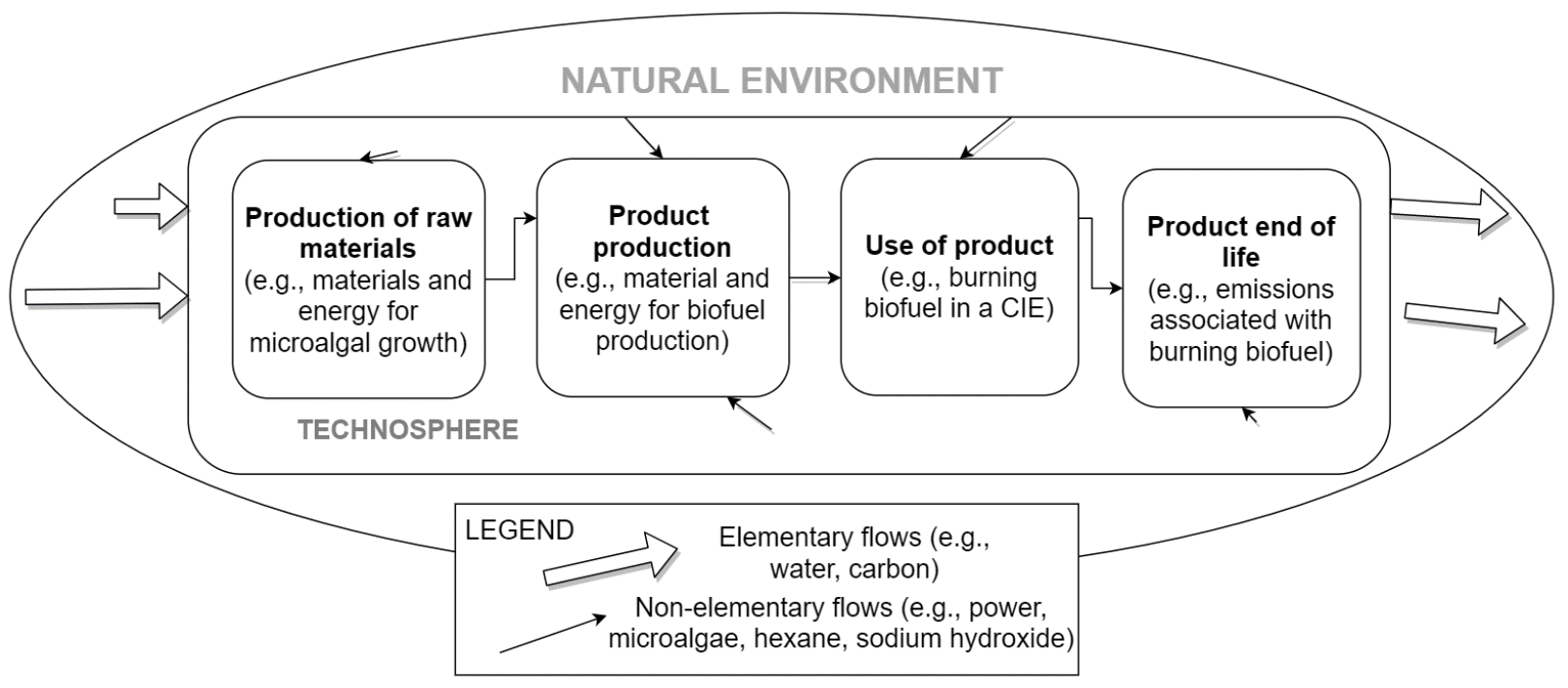

Figure 2.13: Life cycle of a product or process - adapted from GaBi (Thinkstep GaBi, 2017) 
The flows of material to and from the natural environment are called elementary flows (Thinkstep $\mathrm{GaBi}$, 2017). These flows are the movement of natural material such as water, wood, coal etc to, from and within the natural environment. Non-elementary flows represent the movement of material within the technosphere (Thinkstep GaBi, 2017). This material does not interact with the natural system. For example, steel is manufactured by combining iron, carbon, nickel, titanium and potentially other metals depending on the steel characteristics required. This manufacturing process produces an elementary flow that draws material from the natural environment. The steel then flows (i.e., a non-elementary flow) or moves to the use phase where it is used to build a structure. This non-elementary flow also requires energy and the use of other materials. At some point however, steel will reach the end of its life and will need to be recycled or reduced to its natural components in order for these components to return to the natural environment as elementary flows.

A life cycle assessment quantifies both the elementary flows and the non-elementary flows associated with the production of a product or process (Thinkstep GaBi, 2017). As all of these flows require energy and material, the amalgamation and quantification of these flows determine the overall impact of the product's life cycle. When looking at any kind of impact (e.g., environmental), the inputs and outputs of a product or process are quantified based on the impact category. Table 2.12 includes different impact categories (e.g., climate change, energy use) and their associated units of measurement. If, for example, climate change were an impact category requirement as part of the life cycle assessment of a product, the quantity of all material and energy used to produce this product as well as the material and energy required to recycle and dispose of it, in terms of elementary and non-elementary flows, would be acquired. Then these values would be converted to the unit associated with the impact category "Climate Change", which is kilograms of equivalent carbon dioxide $\left(\mathrm{kg} \mathrm{CO}_{2} \mathrm{e}\right)$ (International Organization for Standardization, 2006a). The amalgamation of all the elementary and non-elementary flows in terms of $\mathrm{g} \mathrm{CO}_{2} \mathrm{e}$ would represent the impact that this product's life cycle has on the natural environment, specifically in terms of Climate Change (International Organization for Standardization, 2006a). 
Table 2.12: Examples of key LCA variables associated with environmental impacts (Bare et al., 2000; International Organization for Standardization, 2006a)

\begin{tabular}{|l|l|l|l|l|}
\hline LCA Variables & Definition & \multicolumn{3}{|l|}{} \\
Category & $\begin{array}{l}\text { Environmental } \\
\text { concerns to which Life } \\
\text { Cycle Impact } \\
\text { Assessment results are } \\
\text { assigned }\end{array}$ & $\begin{array}{l}\text { Climate } \\
\text { Change }\end{array}$ & $\begin{array}{l}\text { Fossil } \\
\text { depletion }\end{array}$ & Energy use \\
\hline $\begin{array}{l}\text { Impact } \\
\text { Category } \\
\text { Indicator }\end{array}$ & $\begin{array}{l}\text { Environmental result } \\
\text { of impact category } \\
\text { concern }\end{array}$ & $\begin{array}{l}\text { Increased } \\
\text { Radiative } \\
\text { Forcing }\end{array}$ & Scarcity & Consumption \\
\hline $\begin{array}{l}\text { Categorization } \\
\text { knoctor (also } \\
\text { point category*) }\end{array}$ & $\begin{array}{l}\text { A quantifiable } \\
\text { representation of an } \\
\text { impact category in } \\
\text { order to aggregate } \\
\text { results within the } \\
\text { impact category }\end{array}$ & $\begin{array}{l}\text { Global } \\
\text { Warming } \\
\text { Potential }\end{array}$ & $\begin{array}{l}\text { Displaced } \\
\text { quantity }\end{array}$ & $\begin{array}{l}\text { Quantity } \\
\text { consumed }\end{array}$ \\
\hline Units & $\begin{array}{l}\text { Measurement of the } \\
\text { categorization factor }\end{array}$ & $\begin{array}{l}\text { kg CO } 2 \text { (based } \\
\text { on energy, } \\
\text { renewable or } \\
\text { otherwise) }\end{array}$ & kg of oil eq & $\begin{array}{l}\text { MJ of energy } \\
\text { (renewable } \\
\text { or otherwise) }\end{array}$ \\
\hline $\begin{array}{l}\text { End-point } \\
\text { category }\end{array}$ & $\begin{array}{l}\text { Ultimate impact of the } \\
\text { impact categories } \\
\text { and ecosystem } \\
\text { quality }\end{array}$ & $\begin{array}{l}\text { Resource } \\
\text { availability }\end{array}$ & $\begin{array}{l}\text { Resource } \\
\text { availability }\end{array}$ \\
\hline
\end{tabular}

Three main areas of variance differentiate environmental microalgal BD LCAs. These areas of variance are process pathways, boundary selection and co-product impact allocation (Quinn and Davis, 2015). Co-products are the multitude of products produced in consort with BD from microalgae. These include fertilizer, animal feed, pigments, solvents, pharmaceuticals and other biofuels such as bio-methane, bio-ethanol and bio-butanol (Broch et al., 2014; Maddi et al., 2016; Wiley et al., 2011).

The differences between microalgal BD process pathways in LCA studies can allow for comparison between different processes, which is very useful for individuals trying to determine which process has the least environmental impact. However, boundary differences and coproduct allocation differences can make it more difficult to make comparisons between studies. The following sections describes the areas of variance in microalgal BD LCA models and present the most current LCA microalgal BD studies and their associated climate change 
impacts. The section also includes some RD and LS diesel climate change data for context and comparison purposes.

\subsubsection{Process pathways}

Processes used to produce microalgal BD and similar co-products differ between LCA microalgal biodiesel studies. This variance is valuable because it allows for the comparison between process chains. For example, Frank et al. (2013) completed a study that compared the production of RD using a lipid extraction (LE) method to the production of RD using a hydrothermal liquefaction (HTL) method. Both processes used the same microalgae grown in an open pond with the same harvesting methods (i.e., DAF and centrifuge). Both processes subjected the wet microalgae to high-pressure homogenization. After homogenization, one process used hexane extraction for the LE method and the other process used hydrothermal liquefaction for the HTL method. The aqueous product from both methods went to a catalytic hydrothermal gasification (CHG) processor to produce biogas and $\mathrm{CHG}$ aqueous product. The aqueous product cycled back to the pond for nutrient supplementation and the biogas went to a co-generation facility to produce power. The final stage of the process upgraded the bio-oil from both LE and HTL processes using hydrogen. The LE bio-oil and the HTL bio-oil required different amounts of hydrogen due to their different amounts of oxygen and nitrogen in the product fuel. In this example, it is easy to compare the climate change impact of the LE and HTL processes to create RD. As seen in Table 2.13, the overall GHG emissions of the LE process $\left(3.7 \mathrm{kgCO}_{2} \mathrm{e}\right)$ is less than that of the HTL process $\left(5.4 \mathrm{kgCO}_{2} \mathrm{e}\right)$.

Table 2.13: Frank et al. (2013) GHG results for LE and HTL methods to produce RD

\begin{tabular}{|l|l|l|}
\hline Study Parameters & Net GHG Emissions & $\begin{array}{l}\text { Net GHG Emissions (based on 183 } \\
\text { MJ or 100km travelled in a } \\
\text { compact CIE vehicle) }\end{array}$ \\
\hline $\begin{array}{l}\text { WTW, from LE (Lipid Extracted } \\
\text { Algae), biogas co-product }\end{array}$ & $\begin{array}{l}21.50 \mathrm{kgCO}_{2} \mathrm{e} \text { per MMBTU } \\
(1055 \mathrm{MJ})\end{array}$ & $\mathbf{3 . 7}$ kgCO2e \\
\hline $\begin{array}{l}\text { WTP, sequestration included, from } \\
\text { LEA, biogas co-product }\end{array}$ & $-56.00 \mathrm{kgCO}_{2} \mathrm{e}$ per MMBTU & $-9.7 \mathrm{kgCO} 2 \mathrm{e}$ \\
\hline PTW, combustion only, from LEA & $77.5 \mathrm{kgCO} 2 \mathrm{e}$ per MMBTU & $13.44 \mathrm{kgCO} 2 \mathrm{e}$ \\
\hline
\end{tabular}




\begin{tabular}{|l|l|l|}
\hline $\begin{array}{l}\text { WTW, HTL (Hydrothermal } \\
\text { Liquefaction), biogas co-product }\end{array}$ & $31.00 \mathrm{kgCO}_{2}$ e per MMBTU & $\mathbf{5 . 4} \mathbf{~ k g C O 2 e}$ \\
\hline $\begin{array}{l}\text { WTP, sequestration included, from } \\
\text { HTL, biogas co-product }\end{array}$ & $-46 \mathrm{kgCO} 2 \mathrm{e}$ per MMBTU & $-7.8 \mathrm{kgCO} 2 \mathrm{e}$ \\
\hline PTW, combustion only, from HTL & $77.5 \mathrm{kgCO} 2 \mathrm{e}$ per MMBTU & $13.44 \mathrm{kgCO} 2 \mathrm{e}$ \\
\hline
\end{tabular}

\subsubsection{Boundary selection}

Boundary selections also differentiate microalgal biodiesel and co-product production LCA studies. As seen in Table 2.13, a well to wheel (WTW) boundary differs from a well to pump (WTP) and a pump to wheel (PTW) boundary. The WTW boundary is synonymous with the entire life cycle of a fuel product: production to end of life. The WTP boundary is synonymous with the portion of the life cycle of a fuel from production to the pump station, before it is used for transportation purposes. The PTW boundary is synonymous with a fuel's use and end of life stage (i.e., consumed in a combustion engine). These boundaries need to be the same for effective comparisons.

There are also other ways boundaries in LCA studies can differ that lead to misleading comparisons. For example, Yuan et al. (2015) completed a WTP study assessing the GHG and energy impact associated with producing microalgal $\mathrm{BD}$ and co-products without including infrastructure construction energy, infrastructure and equipment material impacts, maintenance of operation equipment impact, or waste management energy (Yuan et al., 2015). Most microalgal BD studies do not include life cycle impacts other than operational impacts (see Table 2.14 title). The reason for this boundary cut off includes study time constraints or corporate knowledge constraints. Regardless, each LCA study needs to identify these boundary constraints in order for that study to be used comparatively.

\subsubsection{Co-product allocation}

Co-product allocation variation between microalgal BD studies can further complicate the comparison results if it is not clear in the calculations how these co-products contributed to the overall environmental impacts. If a researcher cannot distinguish the impact of the co-product from the overall results, it limits how the researcher can compare the study with another. For 
example, Sander \& Murthy (2010) presented all the GHG impacts associated with each stage of the microalgal BD production process (Table 4 of Sander \& Murthy, 2010). Total GHG impact of the microalgal $\mathrm{BD}$ process is $253 \mathrm{kgCO}_{2} \mathrm{e}$ per 1,000 MJ of BD. The study also included the total value of GHG impacts associated with the co-product: $273 \mathrm{kgCO}_{2} \mathrm{e}$ per 1,000 MJ of BD (i.e., co-product credit). This made assessing the GHG impact of the microalgal BD production system separate from the co-product impact simple.

\subsubsection{Microalgal BD LCA GHG impacts}

Overall, studies have concluded that BD from renewable resources has less greenhouse gas impact than diesel due to the sequestration capacity of the fuel biomass (Nanaki \& Koroneos, 2012) (see Figure 3 in reference). As seen in Table 2.14, emissions produced from WTP stages, in the cases of Sander \& Murthy (2010) and Batan et al. (2010), are less than the emissions emitted to produce the similar WTP phases of fossil fuel (see Table 2.15) (Frank et al., 2013; GREET 2017). These results do not necessarily mean that the GHG impact of the production of microalgal biofuel is less than the GHG impact associated with the production of fossil diesel. The sequestration of carbon offsets the overall WTP results in favour of microalgal fuel. Therefore, to make a true comparison between process pathways, the full WTW results are required. Thus for comparison purposes, the full WTW GHG impact values for microalgal biodiesel and fossil diesel must be determined. In the far right column of Table 2.14, fuel combustion GHG emissions were added to WTP study values such that all studies have final WTW values.

For all studies, microalgal BD and associated co-products produce net positive GHG emissions for the full WTW environmental assessments (see Table 2.14). It is expected that there will be some greenhouse gas impact associated with the production of BD as fossil resources are still very much a part of the anthropogenic material and energy systems (Pfromm et al., 2011). For example, Zaimes \& Khanna (2013) found that the WTP least GHG intensive microalgal BD production process used wet extraction to produce $\mathrm{BD}$, power and heat $\left(30 \mathrm{gCO}_{2} \mathrm{e}\right.$ per $\mathrm{MJ}$ of fuel). Whereas, the WTP most GHG intensive process used dry extraction to produce RD in consort with power, fertilizer and propane using $\mathrm{AD}\left(240 \mathrm{gCO}_{2}\right.$ e per MJ of fuel). Overall, 
Zaimes \& Khanna (2013) found total WTW GHG emissions per $100 \mathrm{~km}$ traveled range between 23.4 and $61.9 \mathrm{kgCO}_{2} \mathrm{e}$.

Sander \& Murthy (2010), Batan et al. (2010) and Frank et al. (2011) produce microalgal BD with less GHG impact than fossil diesel. All three studies use different processes, produce different co-products and allocate these co-products differently. Frank et al. (2011) uses a traditional open pond system for microalgal growth whereas Batan et al (2010) uses a polyethylene bag system. Sander \& Murthy (2010) indicate the lipid-extracted biomass will be feedstock for bio-ethanol production. Thus, Sander \& Murthy (2010) use the system expansion method, described in Chapter 3, for co-product allocation to credit the microalgal BD process with the GHG saved by not having to produce the extra feedstock for bio-ethanol production. Similarly, Batan et al. (2010) indicate that the glycerine and lipid extracted algae co-products will be used to market substitute petrochemical glycerine and fish feed respectively. Thus, credit will be given to the microalgal BD process based on the GHG emissions associated with the production of petrochemical glycerine and microalgal fish feed. None of these studies use a PBR system for microalgal growth. As mentioned in section 2.4.1, GHG impact for PBR growth is usually greater. However, given the variability in climate in northern regions, a PBR growing system is not negotiable if consistent microalgal output is required (Chen et al. 2011; Singh 2012).

Batan et al. (2010) and Frank et al. (2011) developed similar models to the one assessed in this study. Hence the model processes for both Batan et al. (2010) and Frank et al. (2011) are outlined in the following paragraphs.

Batan et al. (2010) produces the lowest GHG impact of the three studies $\left(4.2 \mathrm{kgCO}_{2} \mathrm{e}\right.$ per $100 \mathrm{~km}$ driven in a CIE vehicle). Batan et al. (2010) used a pilot scale polyethylene bag pond system. This model is based on a 315 ha facility producing microalgae for 1 year. Microalgal growth is $260 \mathrm{mg} / \mathrm{L} \cdot \mathrm{hr}$ with a $60 \mathrm{wt} \%$ lipid content. The study's pilot plant is located adjacent to a source of $\mathrm{CO}_{2}$ in order to supply $\mathrm{CO}_{2}$ at $2 \%(\mathrm{v} / \mathrm{v})$. The microalgae moves through a flocculation and centrifuge process for harvest. Lipids are extracted using a shear mixer, decanter, hexane/ethanol solvent and distillation units (i.e., for solvent recovery). Counter flow heat exchangers recover any process heat, and transesterification with methanol and sodium methoxide catalyst produce BD. As mentioned above, co-product credits include glycerine and fish feed. Transport and distribution of the BD final product is included in the model. 
Frank et al. (2011) produces the second lowest GHG impact of the three studies mentioned above (10 $\mathrm{kgCO}_{2}$ e per $100 \mathrm{~km}$ traveled in $\mathrm{CIE}$ vehicle). As also mentioned above, Frank et al. (2011) use a pond for microalgal growth. No chemical flocculants are used and bioflocculation and settling are the first harvesting stage. A centrifuge is used to remove approximately $30 \mathrm{wt} \%$ water and a homogenizer is then used to disrupt the wet microalgal cells prior to extraction. No dryer is used. Hexane extracts lipids, and traditional base-catalyzed transesterification produces BD. Lipid-extracted algae moves through an AD to produce power in a CHP facility, and fertilizer. Process energy and heat offset the energy and heat requirements of the process; the heat and energy is subtracted from the total requirements. Nutrient and carbon dioxide recycling is internal with no co-product credits for additional fertilizer produced due to the GHG impact of fertilizer use. Surprisingly, even though a subsidiary of the U.S. Department of Energy, a proponent of wastewater use for microalgal fuels completed this study, it does not use wastewater and instead uses traditional fertilizers (i.e., urea and diammonium phosphate). The study does mention that nutrients from these fertilizers are continually recycled thus substantially reducing the GHG impact.

\subsection{Summary}

Biodiesel and alcohol fuels were the industry standard prior to the expansion of fossil based fuels in the 1950s (Arbor, 1986). Since liquid fuels will continue to be required to some extent into the future (Allan et al., 2010) and the government of Canada is actively looking for ways to reduce $\mathrm{GHG}$, there is reason to reinvent a more bio-based fuel system.

Since the 1980s, BD has been known to be not only readily biodegradable, but capable of use in CIE vehicles without engine modifications (Arbor, 1986). BD biorefineries already exist today in Canada producing BD from other renewable feedstock and thus have the ability to acquire oil from other feedstock, such as microalgae, for BD production (Biodiesel Magazine, 2017).

A variety of microalgal genera are suitable for biofuel production, specifically biodiesel and alcohol fuels, due to high biomass productivity and high lipid and carbohydrate content (see Table 2.4). Even cyanobacteria populating fresh water lakes can be advantageously sourced for 
biofuel production if the infrastructure is there to take advantage of this resource (Karatay \& Dönmez, 2011; Ma, 2016).

A biofuel facility producing microalgae can act concurrently as a source of wastewater treatment, carbon sequestration source and biofuel biomass feedstock source (Bhola et al., 2014; Ma, 2016; Yun et al., 1997). Nutrients such as nitrogen, phosphorus and carbon, would be readily recycled internally for continued microalgal growth without the requirement of additional fertilizers or water. Anaerobic digestion and co-generation equipment already exist in just under $50 \%$ of all wastewater treatment plants in Canada supplying a city of over 150k people (Lackey et al. 2015). Therefore, recycling nutrients and reducing overall operation power requirements would be possible on-site with existing infrastructure.

LCA results on microalgal $\mathrm{BD}$ and co-products indicate that GHG emissions required to produce and use microalgal BD are slowly decreasing and are sometimes less than the GHG emissions require to produce and use fossil diesel (see Table 2.14 and 2.15). Adding ABE production to the microalgal BD production process could further reduce GHG emissions on a per MJ fuel basis. Considering that butanol is an up and coming substitute for gasoline due to its similar LHV and favourable spark ignition engine properties, determining GHG impact of a combined $\mathrm{BD}$ and $\mathrm{ABE}$ process is a suitable first step towards assessing microalgal biofuel processing. 
Table 2.14: Studies that include GHG impact of microalgal BD and associated co-products, no infrastructure or maintenance included, *Note $-17.9 \mathrm{kgCO}_{2} \mathrm{e}$ is the GHG impact associated with the combustion of fuel driving $100 \mathrm{kms}$ (GREET, 2017)

\begin{tabular}{|c|c|c|c|c|}
\hline$\#$ & Study & Study Parameters & Net GHG Emissions & $\begin{array}{l}\text { Net GHG Emissions (based on } \\
183 \text { MJ or } 100 \mathrm{~km} \text { travelled in a } \\
\text { compact CIE vehicle) }\end{array}$ \\
\hline 1 & $\begin{array}{l}\text { Sander \& Murthy } \\
(2010)\end{array}$ & $\begin{array}{l}\text { WTP, pond, lignocellulosic credit for } \\
\text { ethanol plant feedstock, dried feedstock }\end{array}$ & $\begin{array}{l}-20.9 \text { to } 135.7 \mathrm{kgCO}_{2} \mathrm{e} \text { per } \\
1000 \mathrm{MJ}\end{array}$ & $\begin{array}{l}-3.4 \text { to } 25 \mathrm{kgCO}_{2} \mathrm{e} \text { (need to add } \\
\mathrm{CO}_{2} \mathrm{e} \text { associated with combustion) } \\
+17.9 \mathrm{kgCO}_{2} \mathrm{e}=\mathbf{1 4} \text { to } 43 \\
\mathbf{k g C O} \mathbf{C}(\mathbf{W T W})\end{array}$ \\
\hline 2 & Batan et al. (2010) & $\begin{array}{l}\text { WTP, bag pond (pilot plant scale reactor } \\
\text { system), transesterification, transport, } \\
\text { glycerine and biomass co-product credit, } \\
\text { lipid content } 60 \mathrm{wt} \% \text {, growth rate } 260 \\
\mathrm{mg} / \mathrm{L} \cdot \mathrm{hr}\end{array}$ & $-75 \mathrm{kgCO}_{2} \mathrm{e}$ per $1000 \mathrm{MJ}$ & $\begin{array}{l}-13.7 \mathrm{kgCO}_{2} \mathrm{e}\left(\text { add } \mathrm{CO}_{2} \mathrm{e}\right. \\
\text { associated with combustion })+ \\
17.9 \mathrm{kgCO}_{2} \mathrm{e}=\mathbf{4 . 2} \mathbf{~} \mathbf{k C O}_{2} \mathrm{e} \\
(\mathbf{W T W})\end{array}$ \\
\hline 3 & $\begin{array}{l}\text { Brentner et al. } \\
(2011)\end{array}$ & $\begin{array}{l}\text { WTP, sequestration included, direct } \\
\text { transesterification of wet algal cells with } \\
\text { supercritical methanol, biogas from LEA }\end{array}$ & $\begin{array}{l}805 \mathrm{kgCO}_{2} \mathrm{e} \text { per } 10^{4} \mathrm{MJ}(80 \\
\mathrm{kgCO} 2 \mathrm{e} / 1000 \mathrm{MJ})\end{array}$ & $\begin{array}{l}14.6 \mathrm{kgCO}_{2} \mathrm{e}\left(\text { need to add } \mathrm{CO}_{2} \mathrm{e}\right. \\
\text { associated with combustion) }+ \\
17.9 \mathrm{kgCO}_{2} \mathrm{e}=\mathbf{3 2 . 5} \text { kgCO} \mathbf{e} \\
(\mathbf{W T W})\end{array}$ \\
\hline 4 & $\begin{array}{l}\text { Zaimes \& Khanna } \\
\text { (2013) }\end{array}$ & $\begin{array}{l}\text { WTP, pond, direct inject flue, flocc, } \\
\text { chamber filter press for harvest, waste heat } \\
\text { drying, dry extraction, CHP for residual, } \\
\text { co-products glycerine, power, heat }\end{array}$ & $\begin{array}{l}30-240 \mathrm{gCO}_{2} \mathrm{e} \text { per MJ fuel (30- } \\
\left.240 \mathrm{kgCO}_{2} \mathrm{e} / 1000 \mathrm{MJ}\right)\end{array}$ & $\begin{array}{l}9.2 \mathrm{kgCO}_{2} \mathrm{e} \text { (need to add } \mathrm{CO}_{2} \mathrm{e} \\
\text { associated with combustion) }+ \\
17.9 \mathrm{kgCO}_{2} \mathrm{e}=\mathbf{2 7 . 1} \mathbf{~ k g C O}_{2} \mathrm{e} \\
(\mathbf{W T W})\end{array}$ \\
\hline 5 & $\begin{array}{l}\text { Stephenson et al. } \\
\text { (2010) }\end{array}$ & WTW, biogas co-product credit & $\begin{array}{l}11.9 \times 10^{\wedge} 3 \mathrm{kgCO} 2 \mathrm{e} \text { per } 907 \mathrm{~kg} \\
\text { of } \mathrm{BD}\end{array}$ & $64.5 \mathrm{kgCO}_{2} \mathrm{e}$ \\
\hline 6 & Yuan et al. (2015) & $\begin{array}{l}\text { WTP (cradle to gate), pond, wet extraction, } \\
\text { transport bio oil for conversion to BD, co- } \\
\text { product credits }\end{array}$ & $\begin{array}{l}\text { 72-367 } \mathrm{gCO}_{2} \mathrm{e} \text { per } \mathrm{MJ} \text { of BD } \\
\left(72-367 \mathrm{kgCO}_{2} \mathrm{e} / 1000 \mathrm{MJ}\right)\end{array}$ & $\begin{array}{l}13-67 \mathrm{kgCO}_{2} \mathrm{e} \text { (need to add } \mathrm{CO}_{2} \mathrm{e} \\
\text { associated with combustion) }+ \\
17.9 \mathrm{kgCO}_{2} \mathrm{e}=\mathbf{3 1 - 8 5} \mathbf{k g C O} \mathbf{e} \\
\text { (WTW) }\end{array}$ \\
\hline 7 & Frank et al. (2011) & WTW, pond, AD, co-gen & $55.4 \mathrm{kgCO}_{2} \mathrm{e}$ per $1055 \mathrm{MJ}$ & $10 \mathrm{kgCO}_{2} \mathrm{e}$ \\
\hline
\end{tabular}


Table 2.15: Low Sulfur Diesel life cycle GHG impacts

\begin{tabular}{|c|c|c|c|c|}
\hline$\#$ & Study & Study Parameters & Net GHG Emissions & $\begin{array}{l}\text { Net GHG Emissions (based on } \\
183 \mathrm{MJ} \text { or } 100 \mathrm{~km} \text { travelled in a } \\
\text { compact CIE vehicle) }\end{array}$ \\
\hline 1 & Frank et al. (2013) & $\begin{array}{l}\text { WTW for Low Sulfur Fossil Diesel } \\
\text { (extraction and combustion) }\end{array}$ & $\begin{array}{l}100.00 \mathrm{kgCO}_{2} \text { e per MMBTU } \\
(1055 \mathrm{MJ})\end{array}$ & $17.3 \mathrm{kgCO}_{2} \mathrm{e}$ \\
\hline 2 & Frank et al. (2013) & PTW for LSD (combustion only) & $77.5 \mathrm{kgCO}_{2} \mathrm{e}$ per MMBTU & $13.44 \mathrm{kgCO}_{2} \mathrm{e}(78 \%)$ \\
\hline 3 & Frank et al. (2013) & WTP for LSD (extraction only) & $22.5 \mathrm{kgCO}_{2} \mathrm{e}$ per MMBTU & $3.9 \mathrm{~kg} \mathrm{CO} \mathrm{CO}_{2} \mathrm{e}(22 \%)$ \\
\hline 4 & GREET (2017) & $\begin{array}{l}\text { WTW for Low Sulfur Fossil Diesel } \\
\text { (extraction and combustion) }\end{array}$ & $373 \mathrm{gCO}_{2} \mathrm{e} / \mathrm{mile}$ & $23.3 \mathrm{kgCO}_{2} \mathrm{e}$ \\
\hline 5 & GREET (2017) & PTW for LSD (combustion only) & $286 \mathrm{gCO}_{2} \mathrm{e} / \mathrm{mile}$ & $17.9 \mathrm{kgCO}_{2} \mathrm{e}(77 \%)$ \\
\hline 6 & GREET (2017) & WTP for LSD (extraction only) & $87 \mathrm{gCO}_{2} \mathrm{e} / \mathrm{mile}$ & $5.4 \mathrm{kgCO}_{2} \mathrm{e}(23 \%)$ \\
\hline 7 & $\begin{array}{l}\text { Stephenson et al. } \\
\text { (2010) }\end{array}$ & $\begin{array}{l}\text { WTW for Low Sulfur Fossil Diesel } \\
\text { (extraction and combustion) }\end{array}$ & $86 \mathrm{kgCO}_{2} \mathrm{e}$ per $1000 \mathrm{MJ}$ & $15.7 \mathrm{kgCO}_{2} \mathrm{e}$ \\
\hline
\end{tabular}




\section{Chapter 3 - Materials and Methods}

\subsection{Developing the model}

This study looked at creating as close to a closed loop system as possible for microalgal biofuel production. This includes nutrient recycling and waste re-use where possible (see Figure 3.1). Thus, creating a conceptual model where a microalgal production plant is co-located with a wastewater treatment plant was a preference. The co-location would not only take advantage of wastewater for microalgal growth, but also the equipment and operations currently part of a wastewater treatment facility for use as part of the microalgal production plant. This means that the anaerobic digesters and the co-generation facility operate for both wastewater treatment processing and microalgal biomass processing to produce more power, heat and fertilizer because of increased capacity. Another reason for this co-location is to make potential integration of microalgal growth, harvesting and initial processing into existing anthropological processes as seamless as possible, while using biologically based processes and avoiding the use of environmentally invasive chemicals.

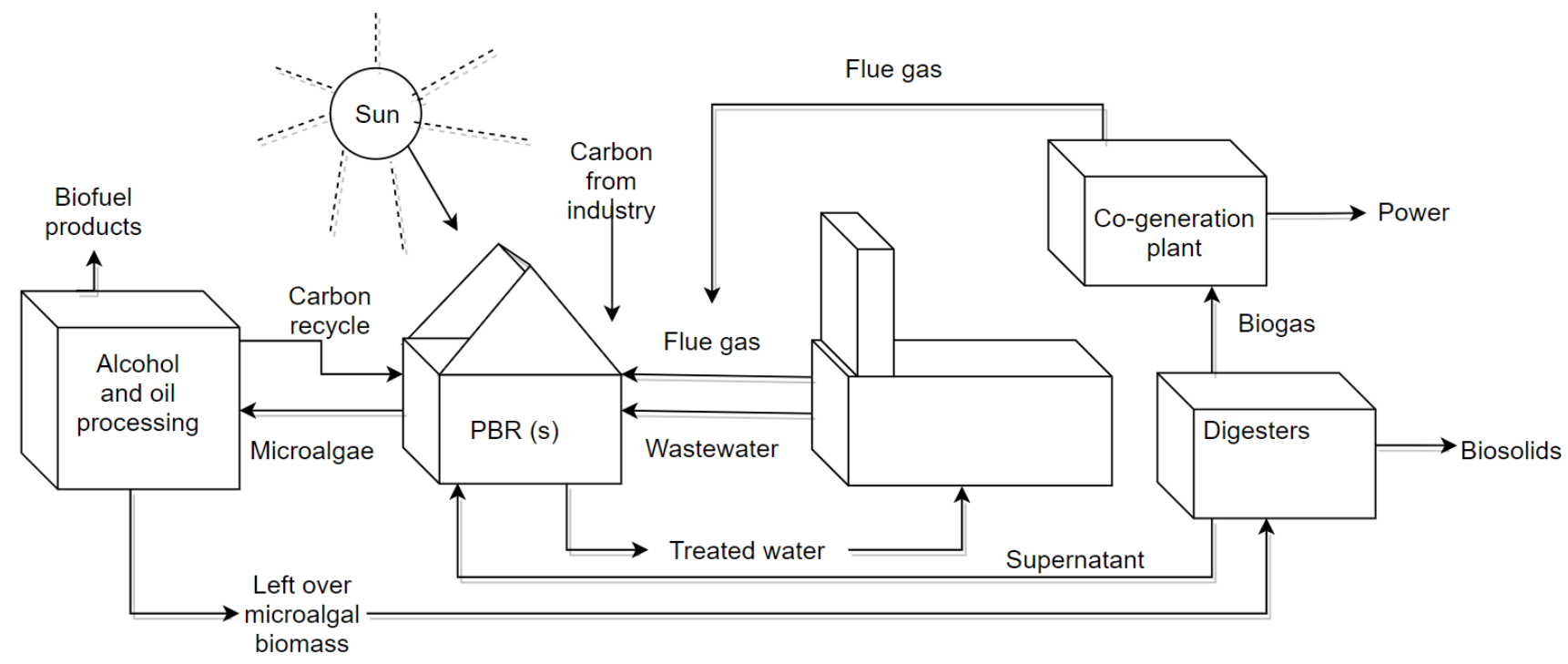

Figure 3.1: Conceptual model of carbon fixation using wastewater nutrients - revised from (Yun et al. 1997) 
From here, the study assumed the use of a multitude of microalgal genus as feedstock for biofuel production because a mixed culture is more resilient and can replicate quickly with additional carbon dioxide from flue gas and nutrients from a variety of wastewaters. Using natural combinations of microalgae in mixed cultures would lead to a more sustainable culture over the long run (see section 2.3). Therefore, a generic growth rate and mixed algal culture would be suitable for this study's purpose. This study planned to draw flue gas from the co-generation plant on-site and co-located industrial facilities. As well, wastewater is drawn from the secondary clarifiers of a wastewater treatment plant in order to channel the most nutrients available as well as the clearest water to assist with photosynthetic efficiency (Barrie wastewater treatment facility, 2017).

The review of the literature concerning microalgal biodiesel and alcohol production processes as well as the report from Ryerson University created by Giang et al. (2017), influenced the model's process selection (Giang et al., 2017). Based on this aforementioned review, the reduction of environmentally invasive chemicals in the model was forefront as additional consumption for the processing and transport of materials would likely increase the GHG impact of the model.

The intention was also to determine the overall energy associated with the microalgal BD and co-product life cycle production process. The reason for determining energy in this situation is because usually with the reduction of chemicals comes the increase in energy requirements as heat and power are used to compensate. Determining energy impact might have been possible if this study had used GREET as the life cycle assessment tool. However, because GREET has a relatively narrow process pathway scope, GREET could not be used (Tatum, 2012). In order to include the $\mathrm{ABE}$ process in the overall microalgal $\mathrm{BD}$ and co-product production process, this study used GaBi Education. In GaBi Education, there was no means of determining total energy impact as one of the environmental impact categories. Therefore, this study only assessed climate change impact over the life cycle of the fuels. Notwithstanding, an increase in GHG emissions could be linked to an increase in energy use considering energy sources are still predominantly fossil carbon intensive. 


\subsection{Methods}

\subsubsection{Goal definition}

According to ISO for LCA, the goal and scope definition section of the study require the reasons for carrying out the study, the intended application of the study, including attributional or consequential perspective, and the study's intended audience (International Organization for Standardization, 2006a). The introduction outlines the reasons for carrying out the study, the intended application of the study and the study's audience. The following paragraphs outline additional requirements.

\subsubsection{Scope definition}

This section requires identification of the study's reference flow, functional unit (also known as the unit of analysis), impact categories, system boundary as well as any study limitations concerning the above mentioned choices (ISO 2006a).

\section{Functional Unit and Reference Flow}

The functional unit (FU) is the quantified purpose of a system for use as a reference unit (ISO 2006a). Several biodiesel LCAs use other units and quantities to compile LCI data and then use the FU to normalize the results in order to represent study objectives and allow for result comparison with other studies. For example, Yuan et al. (2015) focused on mass balance analysis of nutrients, water and carbon for microalgal biodiesel using $1 \mathrm{~kg}$ of dry biomass as a model unit but presents results per MJ of microalgal biodiesel for easy comparison with other fuel studies. For this study, the process is quantified using mass and energy of reactants and products in order to calculate GHG and energy for all life-cycle stages. However, because the purpose of this study's process is to produce biofuel for the transportation system, this study amalgamates all the impacts for all parts of the products' life cycle in reference to the distance travelled in a vehicle.

According to the GHG protocol, a well-defined functional unit consists of three general parameters: the magnitude of the function or service, the duration of the service of that function 
or service and the expected level of quality of that function or service (Sinden et al., 2010). Therefore, the functional unit for this study is one hundred VKT (vehicle kilometer travelled) in a compact diesel car using microalgal BD, bio-acetone, bio-ethanol and bio-butanol. The quality of the fuel is not to ASTM standards; however, as final fuel refinement is not included. See literature review for ASTM fuel properties and the last paragraph in this section 3.2.2 for the justification of the absence of fuel refining in this study.

The reference flow is the measure of the outputs from the process in a given product system required to fulfill the function expressed by the functional unit (ISO 2006a). In reality, the reference flow sets up the process required to produce the common functional unit of two comparable systems such that both these systems can be compared on a relatively equal basis (Weidema et al. 2004). Similar LCA microalgal biodiesel studies have used an overall output or capacity in order to estimate a timeframe to produce a certain volume of product and to quantify associated equipment and materials. Gnansounou \& Raman (2016) used the production of 100,000 tones/year of microalgal biomass as their baseline capacity for analysis (Gnansounou \& Raman 2016). Collet et al. (2011) used 100 ha of cultivated area and 23,000 $\mathrm{m}^{3}$ of total digester volume as their baseline capacity of analysis (Collet et al. 2011). This study's reference flow is the production of approximately 10,000 tonnes (or $10,000,000 \mathrm{~kg}$ ) of microalgae to produce an associated amount of biofuel. The system boundary sub-section and the life cycle inventory analysis (section 3.2.3-4) outline the reference flow in detail. The comparable reference flow is the WTW life cycle of other microalgal BD production processes and/or the traditional WTW life cycle of fossil diesel and associated co-products of a petroleum refinery.

Impact category

The focus of this study is assessing the climate change impact of a microalgal $\mathrm{BD}$ and $\mathrm{ABE}$ production process. Thus, the impact category for this study is climate change only (see Table 2.12 in section 2.5). This limited impact category selection is unfortunate yet typical of most studies using LCA methods (Collotta et al., 2016). However, even though this study does not include other impact categories, such as eutrophication or ozone depletion, for assessment, this study has taken into account the recommendations of other microalgal-energy system LCAs concerning environmental improvements and created a microalgal BD and ABE production 
model accordingly. For example, high energy and environmental impact of microalgal biomass growth associated with nutrient requirements and land area requirements respectively has been accounted for by wastewater use and PBR use (Collotta et al., 2016). Little waste is generated in this process and the use of chemicals is minimalized (e.g., use of energy instead of large volumes of non-recyclable chemicals). Furthermore, the climate change impact category does account for more than just ecosystem risk. According to the JRC for Environment and Sustainability, the climate change impact category's endpoint indicator is human health as well as ecosystem quality (Hunter, 2017). This indicates that the climate change impact category does account for risk to human health (e.g., increase in infectious diseases due to increase in temperature, increase flooding, heat stress) as well as ecosystem risk (e.g., changing biomass, decreasing biodiversity) (Joint Research Centre for Environment and Sustainability, 2011). Whereas, other impact categories measure human health impacts or ecosystem impacts but not both (Hunter, 2017; Joint Research Centre for Environment and Sustainability, 2011).

The unit for the climate change impact category is $\mathrm{kgCO}_{2} \mathrm{e}$. Each greenhouse gas, other than carbon dioxide, has a global warming potential used to derive the GHG impact (i.e., radiative forcing impact) of the GHG based on $\mathrm{kgCO}_{2} \mathrm{e}$. For example, methane is a greenhouse gas and its radiative forcing is relatively 21 times stronger than carbon dioxides over a 100-year period (Qin et al., 2013). Therefore, if a process emitted $10 \mathrm{~kg}$ of methane and 1,000 kg of carbon dioxide, the total impact this resource use would have on climate change would be: $10(21) \mathrm{kgCO}_{2} \mathrm{e}+$ $1,000 \mathrm{kgCO}_{2} \mathrm{e}=1,210 \mathrm{kgCO}_{2} \mathrm{e}$. The GHGs accounted for in this study are predominantly $\mathrm{CO}_{2}$, methane $\left(\mathrm{CH}_{4}\right)$ and nitrous oxide $\left(\mathrm{N}_{2} \mathrm{O}\right)$ (Frank et al. 2011). Sulfur hexafluoride, perfluorocarbons and hydrofluorocarbons are also potent GHGs, however, they are not emitted nearly as often or in the same large quantities. The GaBi Education software has incorporated the GWPs of all associated GHGs based on IPCC protocol (GaBi Thinkstep - PE International, 2017; Qin et al., 2013).

\section{Co-product allocation}

Calculation and subsequent allocation of impacts has notoriously been difficult for LCAs that are capturing the overall impact of a system with several inputs and outputs (Weidema, 2000). Calculating impacts of all inputs and outputs (i.e., waste) for the life cycle of a process under 
study requires the quantification of each input and output's production and distribution. The study then defines these quantities based on the impact category unit and then allocates them to the system's product, or products, if there is more than one product.

If a study has multiple products, similar to this study, the best way to avoid allocation of impacts to multiple products is to quantify the products in terms of a common denominator (e.g., all in terms of MJ of energy). This is only possible if all products are used or can be used in the same capacity. This study groups all biofuel production (i.e., BD and $\mathrm{ABE}$ ) together into one product (i.e., MJ of biofuel energy). All life cycle system impacts are associated with the production of the total biofuel energy produced. This way of quantifying products not only is in line with the overall objective of the system, the production of biofuel, but also prevents the need to distribute GHG emissions and energy at each life-cycle stage between BD, bio-acetone, bio-butanol and bio-ethanol.

At some point, allocation of co-products or waste is required to include co-product benefits to the system and the impacts of waste. There are often co-products that are not of direct use in the system under study, such as biosolid (i.e., fertilizer) in this study's case, but can be used in other systems. There are three ways of calculating and allocating impacts to co-products: 1) subdivision 2) substitution and 3) allocation based on mass, energy or economic value (Collet et al. 2014; Yuan et al., 2015). According to the GHG protocol, allocation methods one through three are preferred in that order (Sinden et al., 2010). There is also the flexibility of using more than one method in a given study if required (Clarens et al., 2011; Stephenson et al., 2010). Note that there are several other terms used to define the same co-product accrediting methods listed above. For example, Frank et al. (2011) use the term displacement for the same method described as substitution in this study. What is important is how the study defines the method(s) used. The following paragraphs define each method.

The subdivision method divides the process under study into sub-processes to the extent that the sub-processes are more easily assigned to a specific product (Sinden et al., 2010; Weidema, 2000). For example, if this study had separated both BD and alcohol products, the study would allocate the SSF process environmental impacts to the alcohol products and not the BD product. This study does not use this method of allocation, as it is not necessary in this study's case. This study uses the substitute co-product allocation method for biosolids and power co-products. The 
following paragraphs explain substitution and allocation based on mass-energy-economic methods.

The substitution method takes the co-product of the system under study and calculates how much impact this product, of given quantity, would have if it were produced traditionally and not in the context of the system under study. This impact is then subtracted from the system under study (Baliga \& Powers, 2010; Bradley et al., 2015; Joint Research Centre for Environment and Sustainability, 2011). This method assumes that this co-product would move into the global material system and reduce its traditional production quantity. Substitution also should quantify the downstream impacts of the co-product (i.e., use in subsequent manufacturing or end of life use) and includes it in the overall impact of the study's main products (Collet et al., 2011). This substitution method may not be feasible, however, if there is no traditional process or if traditional process impact data is not readily available (Azapagic \& Clift, 1999).

This study uses substitution for the biosolid and power co-product to credit the microalgal BD and $\mathrm{ABE}$ production process. For the biosolid co-product allocation, one $\mathrm{kg}$ of nitrogen and $1 \mathrm{~kg}$ of phosphorus from solid digestate $(\mathrm{AD})$ is substituted for $0.6 \mathrm{~kg}$ of nitrogen and $0.4 \mathrm{~kg}$ of phosphorus in synthetic fertilizer respectively (Yuan et al., 2015). This study subtracts the GHG impact associated with producing the equivalent synthetic fertilizer from the overall GHG impact of the overall process. By displacing the product produced traditionally in the market, the production environmental impacts of the traditional product is avoided (Weidema, 2000). In this study, because of the marginal biosolid impact compared to the overall GHG impact of the study, the PBR stage received the credit. However, considering this study has only one product, studies of this type would normally subtract this co-product credit from the total GHG impact and would not selectively benefit the GHG impact of a particular stage of the overall process (Sander \& Murthy, 2010).

This study also uses substitution for the allocation of the co-product, power. This method dictates that this study take the amount of power generated by the co-generation facility and calculates its GHG impact as if the power had been generated by traditional power generation systems. This study should then subtract this GHG impact from the total GHG impact of the process. However, since the overall process of this study is broken down into stages, this study takes the total power produced (i.e., $723 \mathrm{kWh}$ ) and breaks it down based on the percentage of 
how much electrical power each process stage requires (see Appendix A, IO for Anaerobic Digester for calculations). Then each process's electrical energy requirement decreases based on this percentage of the total power produced. This is equivalent to subtracting the GHG associated with power production from the each stage of the process.

Co-product impact allocation based on mass, energy or economic value is also quite common in microalgal LCAs (Clarens et al. 2011; Collet et al. 2014; Yuan et al., 2015). This method distributes co-product impacts between products based on the mass, energy or economic value of these co-products in relation to the products. For example, Collet et al. (2014) allocated some of the GHG impact of the overall process to their co-products, glycerine and oil cake, based on glycerine and oil cake's energy content in relation to the energy content of the main BD product. This reduced the GHG impact of the main product, BD.

If the co-product allocation method by energy-mass-economics is used, the European Directive on Renewable Energy favours co-product allocation based on energy content as this is deemed more consistent and akin with energy producing systems (Collet et al., 2014; European Parliament and Council of the European Union, 2009). There are also some LCAs that prefer using mass and economic allocation as these are perceived to better reflect socio-economic preference (Brentner et al., 2011; Clarens et al. 2011). However, the ILCD handbook indicates that the use of market-price-based allocation partly or entirely prevents the use (i.e., comparison) of results in eco-efficiency studies since the environmental results are directly correlated with market price (Joint Research Centre for Environment and Sustainability, 2011). These market prices are subject to socio-political fluctuations and only add to the uncertainty of an arguably subjective co-product classification method.

\section{System boundary}

Figure 3.2 is a schematic of this study's scope. Figure 3.2 also depicts the WTW (cradle to grave) life cycle of fossil diesel for reference purposes. Note that this study's environmental life cycle assessment only includes the operational life cycle of the biofuel process. This means that no infrastructure and equipment construction energy, infrastructure and equipment material impacts or maintenance of operation equipment impact is included in this environmental life 
cycle analysis. Only wastewater is a system waste, therefore energy required to process this wastewater is included. Note that the impact associated with the life cycle of the material and energy for this study's process is included. This is similar to those studies outlined in Table 2.14 in section 2.5.4, except for waste treatment. Attempting to complete all infrastructure and operational process equipment impacts would have taken a substantial amount more time, resources and expertise. At the current stage of microalgal BD and ABE process development, it makes more sense to determine operational environmental impacts rather than full scope environmental impacts. A study should consider a WTW assessment including infrastructure and equipment prior to the development of a facility for both environmental and economic reasons.

Biodiesel and ABE

\begin{tabular}{|c|c|}
$\begin{array}{c}\text { 1. Extraction of raw } \\
\text { materials (i.e., operational } \\
\text { materials) }\end{array}$ & $\begin{array}{c}\text { Power generation, } \\
\text { solvent production and } \\
\text { transport }\end{array}$
\end{tabular} \begin{tabular}{c}
$\begin{array}{c}\text { Extraction of crude } \\
\text { oil and transport to } \\
\text { refinery }\end{array}$ \\
\hline
\end{tabular}

2. Manufacturing
(sequestered $\mathrm{CO}_{2} \mathrm{e}$
benefit not included)

3. Distribution
$\begin{gathered}\text { 4. End of Llfe } \\ \text { (burning } \mathrm{CO}_{2} \mathrm{e} \\ \text { impact not included) }\end{gathered}$

\section{Fossil Diesel}

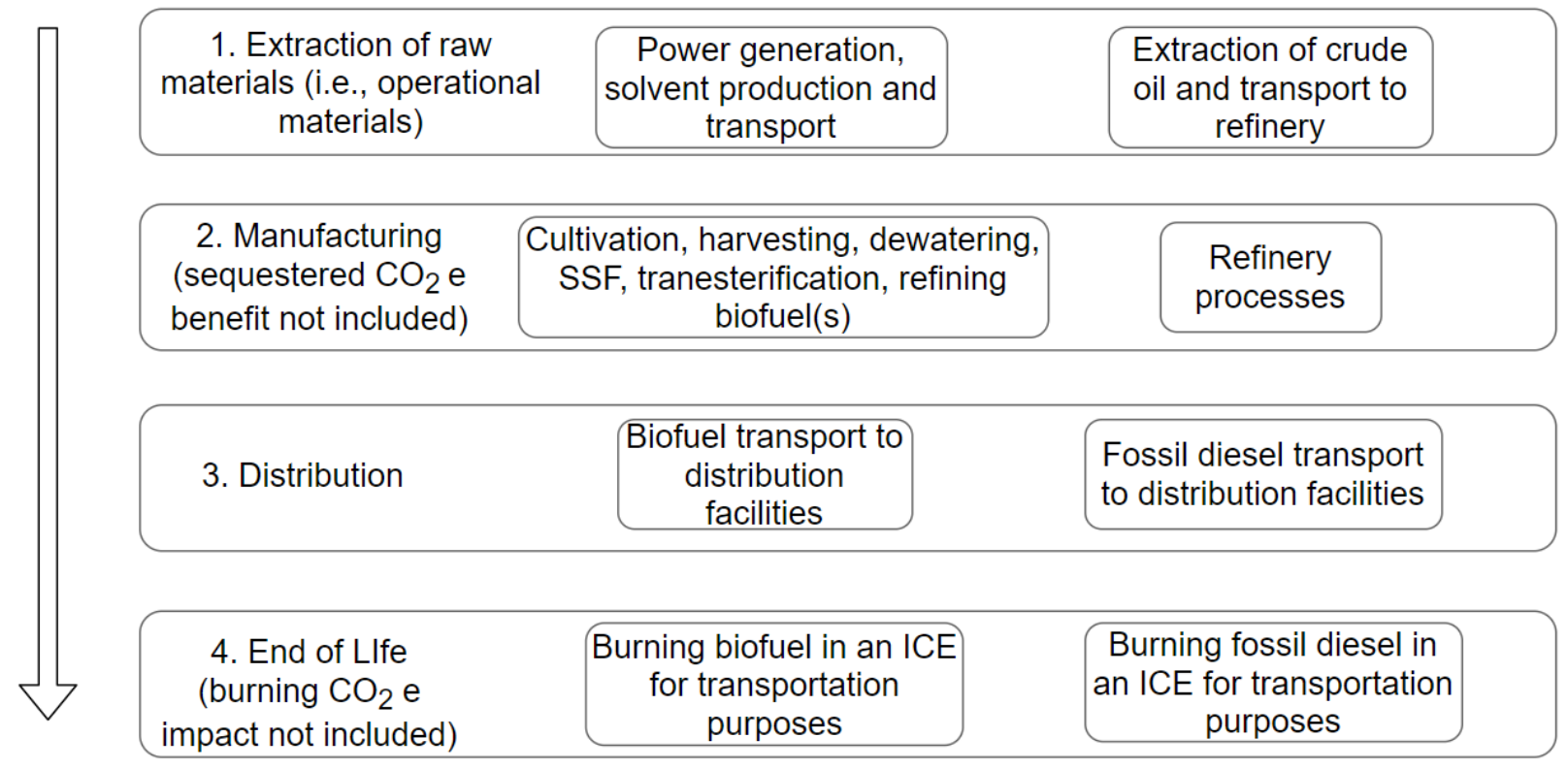

Figure 3.2: Cradle-to-grave scope of this study's operational microalgal biofuel life cycle and the cradle-to-grave scope of the operational fossil diesel's life cycle - revised from (Nanaki \& Koroneos, 2012)

Not including infrastructure in this study's system boundary requires the exclusion of vehicle production in the model. According to GREET 2017, vehicle production produces an additional $37 \mathrm{gCO}_{2} \mathrm{e} / \mathrm{mile}$ or $2.3 \mathrm{kgCO}_{2} \mathrm{e}$ per $100 \mathrm{~km}$ vehicle travel (United States of America Argonne National Laboratory, 2017). This GHG impact would be applicable to the life cycles of both fossil and biodiesel. Therefore, not including this impact is inconsequential to the overall 
assessment. Furthermore, this study does not include additional refining processes associated with final processing of bio-based fuels outlined in 2.4.6. This additional processing would have required the additional use of petrochemicals (solvents) that would have increased the GHG impact of the final product. This impact would be minimal, as the solvents here would be recycled similarly to those used in the main process.

\subsubsection{Study limitations and assumptions}

All assumptions are included in the Appendices preceding calculations. However, the main assumptions and their corresponding study limitations are summarized in the following sections. The Life Cycle Impact Assessment section explains the GaBi Tool limitations.

Process flow and corresponding limitations

This study considers tubular PBRs (approximately 2,800 of them - see Appendix B.1 for calculations) to grow 10,000 tonnes of microalgae in a year. It is understood that growing microalgae in PBRs will be more GHG intense than microalgal grown in open ponds (Quinn \& Davis 2015). Therefore, this study expects higher GHG impacts from the microalgal growth stage than would be apparent otherwise. Microalgae growth is assumed to be $265 \mathrm{mg} / \mathrm{L} \cdot$ day. This value is a conservative growth rate as typically algal growth is less when grown in wastewater (see Table 2.5 and Table 2.8). However, in this case, nutrients are optimized such that this growth rate is reasonable.

The lipid:lignocellulosic:protein ratio by weight in this study's microalgae is 35:35:30. This ratio will vary given a mixed culture environment and the results section of this study will discuss this aspect. This study assumes no algal loss during harvesting and separation, which ultimately decreases the GHG impact per MJ of energy produced. However, this assumption would not affect the results of this study significantly. Although microalgae is small ( 5 micron), a pulveriser is used instead of additional acid or base to assist with the breakdown of interstitial bonds between microalgal components.

Lipid extraction uses hexane and ethanol solvents (Petrick et al., 2013). As this microalgal BD process also produces ethanol, a small amount of this ethanol is redirected and used as a solvent 
in this model, which reduces the need to source and transport additional ethanol. This study assumes $5 \%$ loss of these solvents during operations. This means that only $5 \%$ of the solvent requirement is included in the GHG impact as this is the amount that is required on a regular basis. The harvesting stage and pre-treatment stages of the process use sodium hydroxide and sulfuric acid to neutralize and produce wastewater, which is non-toxic and easily processed as wastewater. Additionally, all material sources for this process are within a $27 \mathrm{~km}$ radius (see Appendix $\mathrm{C}$ for calculations). Transesterification is by supercritical methanol and conversion of TG to FAME is $97 \%$ (Liu, 2013).

Pre-treatment of lignocellulosic biomass includes sulfuric acid bath and heating (Begum \& Dahman, 2015). Subsequently, nitrogen gas circulates materials in the SSF reactor. This study assumes a $10 \%$ loss of nitrogen gas, therefore, similar to solvent assessment above, only $10 \%$ of the overall required amount is included in the GHG impact assessment. This study assumes small amounts of nutrients required to supplement bacteria growth in the SSF and that GHG impact of these nutrients is negligible. This study also does not include the impact associated with the use of replenishment of bacteria for the SSF process. The fused $C b C t$ bacteria are subject to cell immobilization such that replenishment of bacteria occurs infrequently (Dolejš et al., 2014; Green, 2011; Kök, 2016). Eighty two percent of the lignocellulosic biomass is fermented and $49 \%$ of the fermented sugar is converted to ABE (Begum \& Dahman 2015). Distillation of $\mathrm{ABE}$ includes the use of water to assist with the separation of any residual water left in the alcohol stream. This addition of water, once used, is considered wastewater and is included as such in wastewater treatment processing impacts

The top left corner of Figure 3.4 depicts the wastewater equipment used in this process. Colocating this study's biofuel process with a wastewater treatment plant provides nutrients to grow microalgae and allows for nutrient and co-product recirculation. Figure 3.3 is a simplistic diagram of nutrient flow and co-product recirculation within the overall system. The only removal of carbon from the system is through the biosolid and biofuel pathways. Additional carbon is supplemented using industrial flue gas. The following paragraph details Figure 3.3. 
For optimal nutrient recovery and co-product use, the process strips the AD biogas to produce carbon dioxide and methane. Carbon dioxide flows to the PBR to supplement the carbon requirement (flow 1 in Figure 3.3) and methane produces electricity in the plant's gas turbine cogeneration system (flow 7 in Figure 3.3). The electrical power generated from burning methane produced by AD process uses a 30\% efficiency rate (Frank et al. 2011). This electricity offsets the electrical power and heat requirements of the system under study (flow 9). It is assumed that the heat generated by burning methane is sufficient to heat the AD (Frank et al. 2011). The flue gas from the co-generation plant and carbon dioxide from the $\mathrm{ABE}$ fermentation process supplements the carbon requirement of the PBR (flow 8 and 6 respectively). The AD supernatant supplements the nutrient requirements in the PBR (flow 4). This study assumes no nutrient loss and additional nutrients will accumulate in the biosolids produced by the AD. Using the additional nutrients, the equivalent amount of fertilizer is calculated based on the biosolid production rate and credited to the GHG impact of the system. Unreacted lipids, lignocellulosic biomass and protein are digested (flow $2 \& 3$ ). Finally, glycerol from the BD production process is recycled back into the PBR for carbon balance purposes (flow 5).

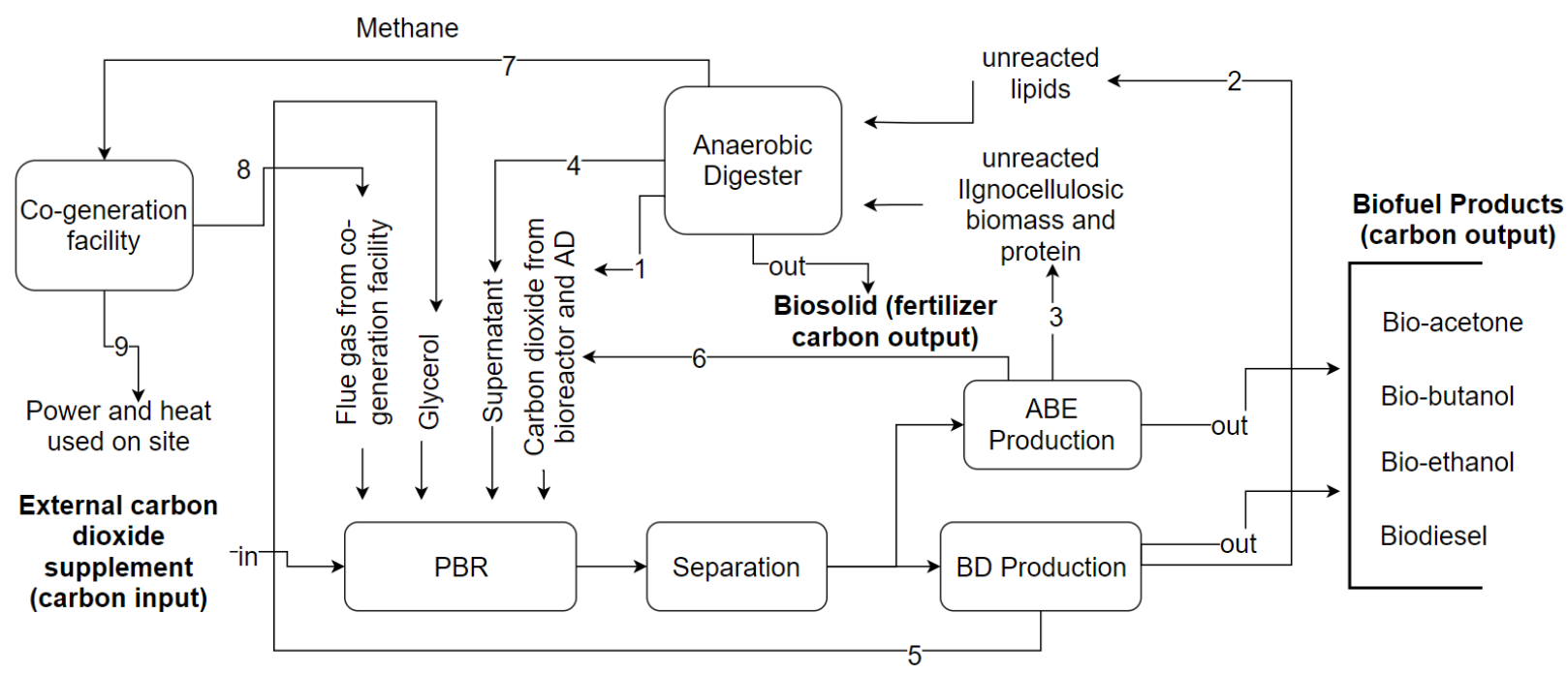

Figure 3.3: Schematic of nutrient and energy balancing (bold text indicates carbon input and output to the system) 
The refrigerants used in this study are Dowthern A and water. Both are continually recycled with no waste treatment required. Any process over $100^{\circ} \mathrm{C}$ uses Dowthern A as the refrigerant to avoid water vaporizing and additional pressure requirements. This study assumes a 5\% loss of refrigerant; therefore, this study uses $5 \%$ of refrigerant requirement to calculate the associated GHG impact.

Based on calculations in Appendix B.2, heating and cooling energy requirements are approximately equal when grouped based on the refrigerant used (see Table B.3 and B.4). Therefore, this study included the GHG impact of the heat energy required only. The cooling energy capacity is not included in the GHG impact. Accordingly, this study uses NG to heat the refrigerant, the refrigerant heats a medium and then the refrigerant subsequently cools another medium before being re-heated. This heating and cooling optimization conserves all heat and mimics an adiabatic system. Thus, this system is very similar to the CHP system used by Frank et al. (2011).

This study did not create a detailed design specification for this process. A design specification includes drawings, dimensions, sizes, specific procedures and maintenance requirements associated with the process equipment. As such, this study did not determine the specifications of each piece of equipment. Consequently, estimations were used for pumps and equipment used to circulate materials. This study assumed half HP pumps to move the microalgal stream from PBRs to the processing system and to move materials between stages of the production process. The size of the pump is reasonable considering the slow rate of movement of most material. Impact on GHG emission results will be greater for cultivation, but less than 5\% of the stage's GHG impact for all other process stages. See Appendix B.1 for circulation power requirements and calculations.

\section{Time frame assumptions and limitations}

This study has the base operating parameters of a fixed amount of microalgal production (i.e., 10,000 tonnes) with the required wastewater volume to produce the required amount of 
microalgae in a 47-week period. In this study, it is assumed that five weeks of the year, the biofuel production system would not be operational for maintenance. Ten thousand tonnes of microalgae per year is reasonable for microalgal growth in an industrial facility at the pilot scale, however it has not been accomplished as of yet with PBRs (i.e., only with open ponds).

The overall time appreciation of 1 year for this study is preferred for two reasons. First, a year timeline for production aligns this study's process with the preferred way of measuring a BD production facility output. This study's approximately 5.7 million liters of fuel output falls in the mid to low range of the current BD production facilities in Canada today (see section 2.1.1). Second, having a yearly output implies a degree of operational stability that makes it easier to calculate hourly outputs from a larger initial volume. There is more accuracy involved when calculating overall large and small quantities of material on a large scale (i.e., a year and annual tonnage) before breaking these values down to a tangible reference (i.e., hourly requirement). For example, this model recycles the solvents methanol, hexane and ethanol. The LCA calculates the GHG impact of these solvents based on how much is required to supplement loss and not based on how much is required at the onset of the production process for a given amount of feedstock or biomass. This way of calculating GHG impact of cyclically used material is reasonable and drastically reduces the GHG impact of the process. It is more accurate to calculate how much solvent is required per year, reduce this to an hourly requirement and then take a percentage of loss from this value. Otherwise, there could be the assumption that the loss is negligible and the study would fail to include GHG impact for this material requirement.

\subsubsection{Life Cycle Inventory Analysis}

Table 3.1 outlines all the material and energy associated with this study's microalgal BD and ABE production process by stage. All calculations for the material and energy use found in Table 3.1 are in Appendix A. Appendix A contains a breakdown of each process stage, also linking all calculations found in these appendices with the process stages depicted in Figure 3.4. 


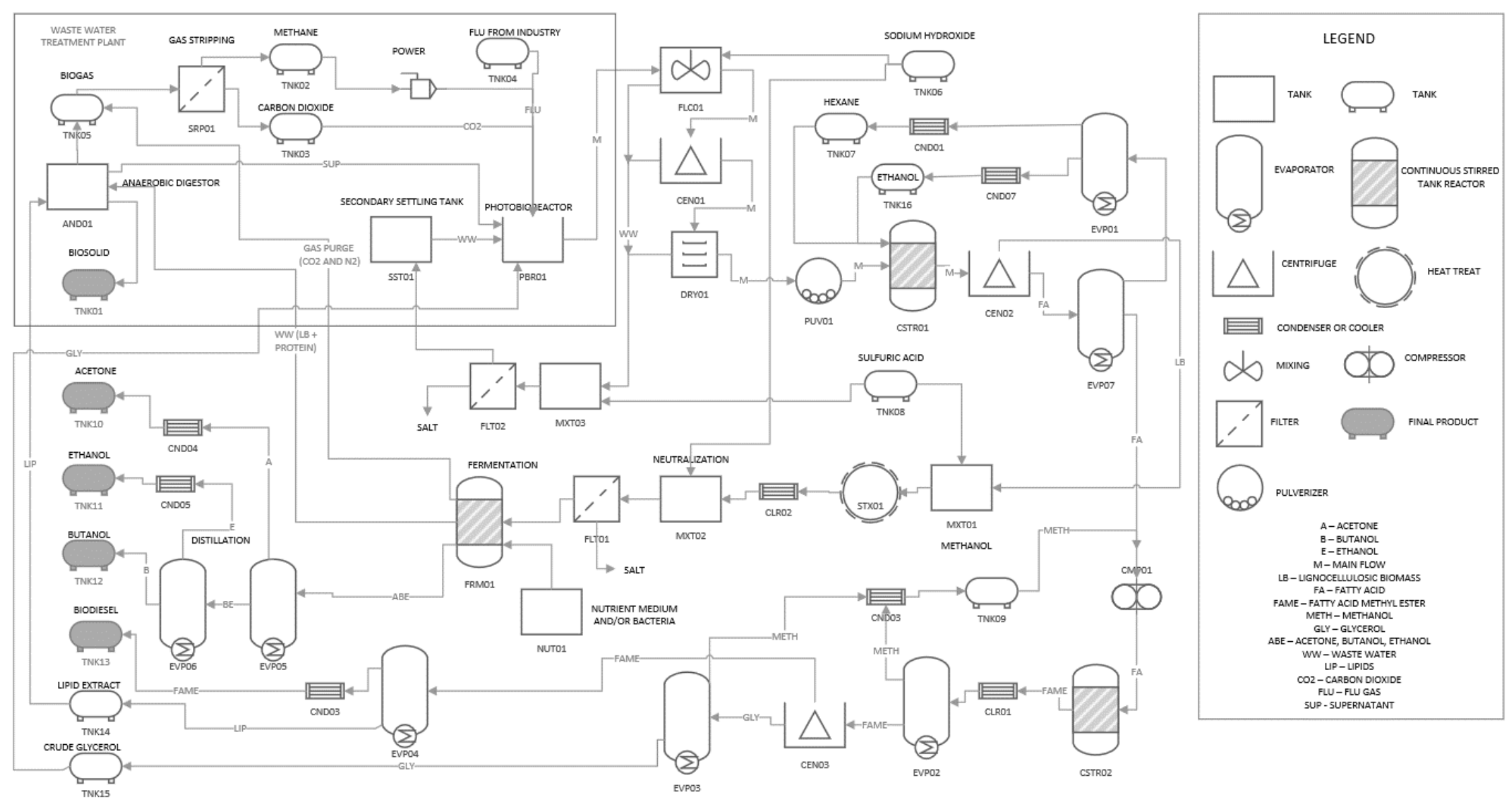

Figure 3.4: Microalgal BD and ABE process flow model (Note - pumps are not included in this diagram. Appendix B.1 contains all pumps with associated energy calculations) 
Table 3.1: Materials required for each stage of the microalgal BD and ABE production process (Appendix A contains all calculations)

\begin{tabular}{|c|c|c|c|}
\hline Stage & Material & $\begin{array}{l}\text { Energy (on a per hour } \\
\text { basis) }\end{array}$ & Amount (on a per hour basis) \\
\hline \multirow[t]{5}{*}{ PBR } & Carbon dioxide & & $1,643 \mathrm{~kg}$ \\
\hline & Wastewatser & & $4.79 \times 10^{\wedge} 6 \mathrm{~L}$ \\
\hline & Internal energy for PBR(s) (electrical) & $13,951 \mathrm{MJ}(3,875 \mathrm{kWh})$ & \\
\hline & $\begin{array}{l}\text { Circulation to/from PBR and plant } \\
\text { (electrical) }\end{array}$ & 3,741 MJ (1,039 kWh) & \\
\hline & & $4,914 \mathrm{kWh}$ & \\
\hline \multirow[t]{7}{*}{ Harvest } & Sodium hydroxide & & $958 \mathrm{~kg}$ \\
\hline & Sulfuric acid & & $1,177 \mathrm{~kg}$ \\
\hline & FLC energy (electrical) & $72 \mathrm{kWh}$ & \\
\hline & CEN energy (electrical) & $5,450 \mathrm{kWh}$ & \\
\hline & DRY energy (NG) & $4,053 \mathrm{kWh}$ & \\
\hline & $\begin{array}{l}\text { Energy for material circulation } \\
\text { (electrical) }\end{array}$ & $11.7 \mathrm{MJ}(3.3 \mathrm{kWh})$ & \\
\hline & & $9,578 \mathrm{kWh}$ & \\
\hline \multirow[t]{11}{*}{ Separation } & PUV energy (electrical) & $241.2 \mathrm{MJ}(67 \mathrm{kWh})$ & \\
\hline & CSTR energy (electrical) & $184.84 \mathrm{kWh}$ & \\
\hline & CSTR heat (NG) & 3,147 MJ (874 kWh) & \\
\hline & Hexane & & $5.99 \mathrm{~kg}$ \\
\hline & Ethanol & & $0.65 \mathrm{~kg}$ \\
\hline & CEN energy (electrical) & $20.1 \mathrm{kWh}$ & \\
\hline & EVP01 heat (NG) & $2,949 \mathrm{MJ}(819 \mathrm{kWh})$ & \\
\hline & EVP07 heat (NG) & $818 \mathrm{MJ}(227 \mathrm{kWh})$ & \\
\hline & $\begin{array}{l}\text { Energy for material circulation } \\
\text { (electrical) }\end{array}$ & $9.1 \mathrm{MJ}(2.5 \mathrm{kWh})$ & \\
\hline & $\begin{array}{l}\text { Energy for heating/cooling fluid } \\
\text { circulation (NG) }\end{array}$ & $1 \mathrm{MJ}(0.265 \mathrm{kWh})$ & \\
\hline & & $2,195 \mathrm{kWh}$ & \\
\hline
\end{tabular}




\begin{tabular}{|c|c|c|c|}
\hline BD production & CMP energy (electrical) & $3.6 \mathrm{kWh}$ & \\
\hline & Methanol & & $49.86 \mathrm{~kg}$ \\
\hline & CSTR heat (NG) & $42.3 \mathrm{kWh}$ & \\
\hline & CEN energy (electrical) & $0.746 \mathrm{kWh}$ & \\
\hline & EVP heat (NG) & $73 \mathrm{kWh}$ & \\
\hline & Dowthern A & & $165.6 \mathrm{~kg}$ \\
\hline & $\begin{array}{l}\text { Energy for material circulation } \\
\text { (electrical) }\end{array}$ & $15.6 \mathrm{MJ}(4.3 \mathrm{kWh})$ & \\
\hline & $\begin{array}{l}\text { Energy for heating/cooling fluid } \\
\text { circulation (NG) }\end{array}$ & $0.5 \mathrm{MJ}(0.147 \mathrm{kWh})$ & \\
\hline & & $124 \mathrm{kWh}$ & \\
\hline ABE production & MXT sulfuric acid & & $175 \mathrm{~kg}$ \\
\hline & MXT water & & $9,573.8 \mathrm{~kg}$ \\
\hline & STX heat (NG) & 4,176 MJ $(1,160 \mathrm{kWh})$ & \\
\hline & MXT sodium hydroxide & & $165 \mathrm{~kg}$ \\
\hline & FRM nitrogen & & $142 \mathrm{~kg}$ \\
\hline & $\begin{array}{l}\text { FRM energy for circ of nitrogen } \\
\text { (electrical) }\end{array}$ & $0.024 \mathrm{kWh}$ & \\
\hline & Energy for stripping nitrogen (electrical) & $0.34 \mathrm{kWh}$ & \\
\hline & EVP water & & $174 \mathrm{~kg}$ \\
\hline & EVP energy for water flow (electrical) & $6.4 \times 10-3 \mathrm{kWh}$ & \\
\hline & EVP heat (electrical) & $13.94 \mathrm{kWh}$ & \\
\hline & $\begin{array}{l}\text { Energy for material circulation } \\
\text { (electrical) }\end{array}$ & $16.9 \mathrm{MJ}(4.7 \mathrm{kWh})$ & \\
\hline & $\begin{array}{l}\text { Energy for heating/cooling fluid } \\
\text { circulation (NG) }\end{array}$ & Included in BD energy & \\
\hline & & $1,179 \mathrm{kWh}$ & \\
\hline $\mathrm{AD}$ and power & AD energy (electrical) & $53.3 \mathrm{kWh}$ & \\
\hline & Stripping energy (electrical) & $80.1 \mathrm{kWh}$ & \\
\hline & & $133 \mathrm{kWh}$ & \\
\hline
\end{tabular}


input 


\subsubsection{Life Cycle Impact Analysis Results}

Most studies rely on an LCA software tool (e.g., GREET, GaBi, SimaPro) and corresponding LCI database (e.g., Ecoinvent, MIRO, GaBi) to produce a desired scope depth. Often the LCA software tool has corresponding databases embedded within the tool. Scope depth refers to the level of detail included in the life cycle assessment. For example, an in-depth life cycle analysis of a fuel might include the life cycles of not only the material required to produce the fuel, but the life cycle of the materials required to produce the materials required to produce the fuel.

Out of the LCAs concerning microalgal BD reviewed, only Sander \& Murthy (2010) applied a $5 \%$ cut off to each impact category, which means the author sourced all of the impact associated with the life cycle of the product without the use of a software tool. The rest, such as Bradley et al. (2015), relied on a life cycle software tool to calculate and include nuanced processes within the study's dictated and defined larger processes. Software packages are essential to include the vast amounts of data required to compile a complete characteristic life cycle assessment, but they inevitably also create a degree of obscurity concerning the inclusion or exclusion of process data twice or more times removed from the primary processes.

Fortunately, software programs such as GaBi allow the user to select the degree depth to which the software will compile impact values. For example, GaBi classifies degree depth based on the boundaries unit process single operation (us-o), cradle-to gate (agg), partially terminated system (p-agg) and avoided product system (aps). This study uses all agg data in order to capture the life cycle impact of not only the material, but also the materials and energy associated with the production of such materials.

GaBi Tool

The GaBi acronym is of German origin and stands for "holistic balancing". GaBi is a software tool supported by PE International and consists of a network of databases; some supported by PE International such as Ecoinvent, and other independent databases. The databases supply key information on millions of processes; information such as material requirements, energy requirements and other key requirements that allow the calculator to calculate the impact these processes have on the environment. The program groups these impacts on the environment into 
what is known as impact categories. One of these impact categories, climate change, is the only impact category used in this study. The unit for the climate change impact category is $\mathrm{kgCO}_{2} \mathrm{e}$ and can also be referred to as global warming potential or green house gas impact.

A scaling value is required in order to obtain results from $\mathrm{GaBi}$ in line with this study's objectives; the GHG impact associated with driving $100 \mathrm{~km}$ in a diesel vehicle. This study uses the following assumptions to calculate the equivalent $100 \mathrm{~km}$ scaling value:

- This study's products (i.e., BD, bio-acetone, bio-butanol and bio-ethanol) all contribute to the total fuel energy output. A study conducted by Wu et al. (2007) included bio-acetone, although not a common fuel, in terms of its energy potential to quantify total energy output of the study's ABE process (Wu et al. 2007).

- $\quad$ Lower heating value for (bio)butanol, (bio)ethanol and (bio)acetone are 33.1, 26.8 and 29.6 MJ/kg respectively (Rakopoulos et al. 2011; Xu et al. 2006)

- $\quad$ Lower heating value of BD is found in Table 2.1

- $\quad 0.65 \mathrm{~kg}$ ethanol $/ \mathrm{hr}$ is subtracted from the total production of ethanol for solvent requirements in the extraction process

- $\quad$ MJ required to move a vehicle $100 \mathrm{~km}$ : 183 MJ (using a diesel vehicle) (Nanaki \& Koroneos, 2012)

Thus, the scaling factor for $\mathrm{GaBi}$ for this study is 0.0078. Appendix D.7 includes scaling factor calculations.

GaBi Tool limitations

The version of GaBi used in this study is GaBi Education. GaBi Education is a free LCA software tool geared for students at the Masters level and below to support the developmental stages of life cycle assessment education and research. Therefore, there are some limitations associated with GaBi Education that would not exist in other versions. The following include a summary of this study's limiting parameters when using GaBi Education.

- This study replaced methanol with natural gas as methanol was not available in the database (Thinkstep representative, 2017). This assumption is reasonable as 
methanol is typically derived from NG, however, the steam reforming process required to add oxygen, to form methanol, is not included. The power and material required for a typical steam reforming process would be required to estimate the GHG impact for a similar part of this process. The time required to do this was deemed unnecessary considering the predicted small GHG impact of this stage when compared with the NG GHG impact. See Appendix A.4 and D.4 for calculations/parameters.

- Heating and cooling fluid, Dowthern A, is replaced with ethylene glycol in GaBi as neither Dowthern A nor Dowthern A's components (diphenyl and diphenyl oxide) are available in GaBi Education. The GHG impact difference is likely minimal considering both processes are relatively energy intensive and both materials are derivatives of fossil resources. However, the environmental impact might be quite different.

- As can be seen in the graphs in Appendix D, some of the process material selected relied on geographic information. On occasion, the appropriate geographic data was not available. For example, most materials and their associated life cycle climate change impact were not available in the Canadian context. Therefore, this study opted for other North American based data (i.e., United States of America). In some cases, as this LCA software tool is German based, North American data was not available either. In this case, this study used generic European Union (EU) data or DE (Denmark) data. This could arguably have huge impacts on results. There would be no way of determining the extent of this impact without the use of other $\mathrm{GaBi}$ versions, which would come at a financial cost of 1,266.00 per year (Thinkstep representative, 2017).

GaBi Results

Table 3.2 summarizes the WTW impact of this study's microalgal BD and ABE production process. Three functional units (i.e., $100 \mathrm{~km}$ traveled in a compact diesel vehicle, 1,000 MJ of fuel energy produced and $1 \mathrm{~kg}$ microalgal biomass produced) were included to facilitate comparison with other studies as required. 
Table 3.2: Total climate change impact using three different functional units

\begin{tabular}{|l|l|l|l|l|}
\hline Stage & $\begin{array}{l}\text { CO2e contribution } \\
\text { (per 100 km } \\
\text { traveled in a diesel } \\
\text { passenger car } \\
\text { equivalent to 183 } \\
\text { MJ) }\end{array}$ & $\begin{array}{l}\text { CO2e } \\
\text { contribution } \\
\text { (per 1,000 MJ) }\end{array}$ & $\begin{array}{l}\text { CO2e } \\
\text { contribution } \\
\text { (per kg } \\
\text { microalgae) }\end{array}$ & \% contribution \\
\hline PBR & $28.1 \mathrm{kgCO} 2 \mathrm{e}$ & $153.7 \mathrm{kgCO}_{2} \mathrm{e}$ & $2.8 \mathrm{kgCO}_{2} \mathrm{e}$ & $37 \%$ \\
\hline Harvest & $38.0 \mathrm{kgCO} 2 \mathrm{e}$ & $207.4 \mathrm{kgCO}_{2} \mathrm{e}$ & $3.8 \mathrm{kgCO}_{2} \mathrm{e}$ & $50 \%$ \\
\hline Separation & $2.0 \mathrm{kgCO} 2 \mathrm{e}$ & $11.0 \mathrm{kgCO}_{2} \mathrm{e}$ & $0.2 \mathrm{kgCO}_{2} \mathrm{e}$ & $2.7 \%$ \\
\hline BD production & $1.9 \mathrm{kgCO} 2 \mathrm{e}$ & $10.2 \mathrm{kgCO}_{2} \mathrm{e}$ & $0.2 \mathrm{kgCO}_{2} \mathrm{e}$ & $2.5 \%$ \\
\hline ABE production & $5.1 \mathrm{kgCO} 2 \mathrm{e}$ & $27.9 \mathrm{kgCO}_{2} \mathrm{e}$ & $0.5 \mathrm{kgCO}_{2} \mathrm{e}$ & $6.7 \%$ \\
\hline $\begin{array}{l}\text { Transport of all } \\
\text { products to } \\
\text { biorefinery or } \\
\text { service station }\end{array}$ & $0.02 \mathrm{kgCO}_{2} \mathrm{e}$ & $0.1 \mathrm{kgCO}_{2} \mathrm{e}$ & $0.002 \mathrm{kgCO}_{2} \mathrm{e}$ & $0.3 \%$ \\
\hline AD and power & $0.6 \mathrm{kgCO}_{2} \mathrm{e}$ & & & \\
\hline Total & $\mathbf{7 5 . 7} \mathbf{k g C O}_{2} \mathrm{e}$ & $3.3 \mathrm{kgCO}_{2} \mathrm{e}$ & $0.06 \mathrm{kgCO}_{2} \mathrm{e}$ & $0.8 \%$ \\
\hline
\end{tabular}

Figure 3.5 is a visual representation of the values in Table 3.2. Note here that it is assumed that the microalgae would sequester the products produced by burning the biofuel. This is not the case in reality because burning fossil fuel and biofuel will produce other gasses other than $\mathrm{CO}_{2}$, such as sulfur dioxides, nitrogen dioxides and particulate matter not sequestered by microalgae during growth. However, other studies have assumed that sequestering and burning cancel out, therefore, this study does as well (Frank et al. 2011). This assumption does not change the cradle to grave boundary of this study and it should be directly compared with other WTW studies. 


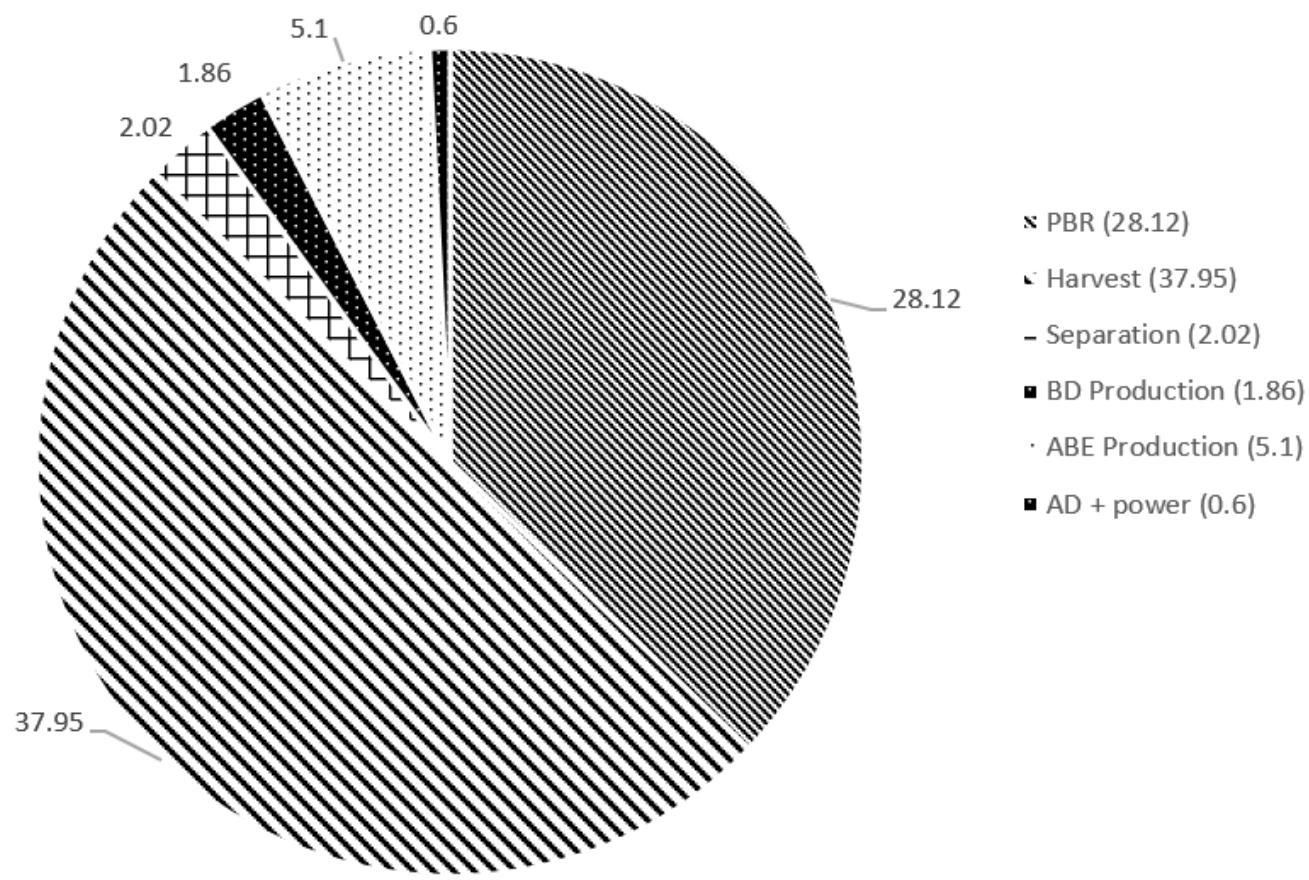

Figure 3.5: Global warming potential contribution in $\mathrm{kgCO}_{2}$ e for this study's microalgal $\mathrm{BD}$ and $\mathrm{ABE}$ production process based on $100 \mathrm{~km}$ driven in a compact diesel vehicle 


\section{Chapter 4 - Results, Discussion and Interpretation}

\subsection{Review of objectives}

As indicated in Chapter 1, the objective of this study is to determine the GHG impact associated with a unique microalgal $\mathrm{BD}$ and $\mathrm{ABE}$ production process in order to compare this process with the other microalgal $\mathrm{BD}$ production processes. There was also a concerted effort made to create a closed loop system where possible to reduce overall material consumption and waste.

Considering the results outlined in 3.2.5 Table 3.2, the following section compares this study's finding with those of other microalgal $\mathrm{BD}$ production processes that have used LCA methods to calculate GHG impact.

\subsection{GHG Results}

As shown in Table 3.2, the cultivation (37\%) and the harvesting (50\%) stages produces the majority of the GHG impact of this microalgal $\mathrm{BD}$ and $\mathrm{ABE}$ production model. Also, the $\mathrm{BD}$ production stage GHG impact was relatively small (2.5\%) compared to the GHG impact of the entire production model.

The GHG impact in each stage of the model is broken down into material, energy and power in the Figure 4.1. The majority of the GHG impact of the cultivation, harvesting and separation stages is from power use. Overall $66 \%$ of the GHG impact is from power use. 


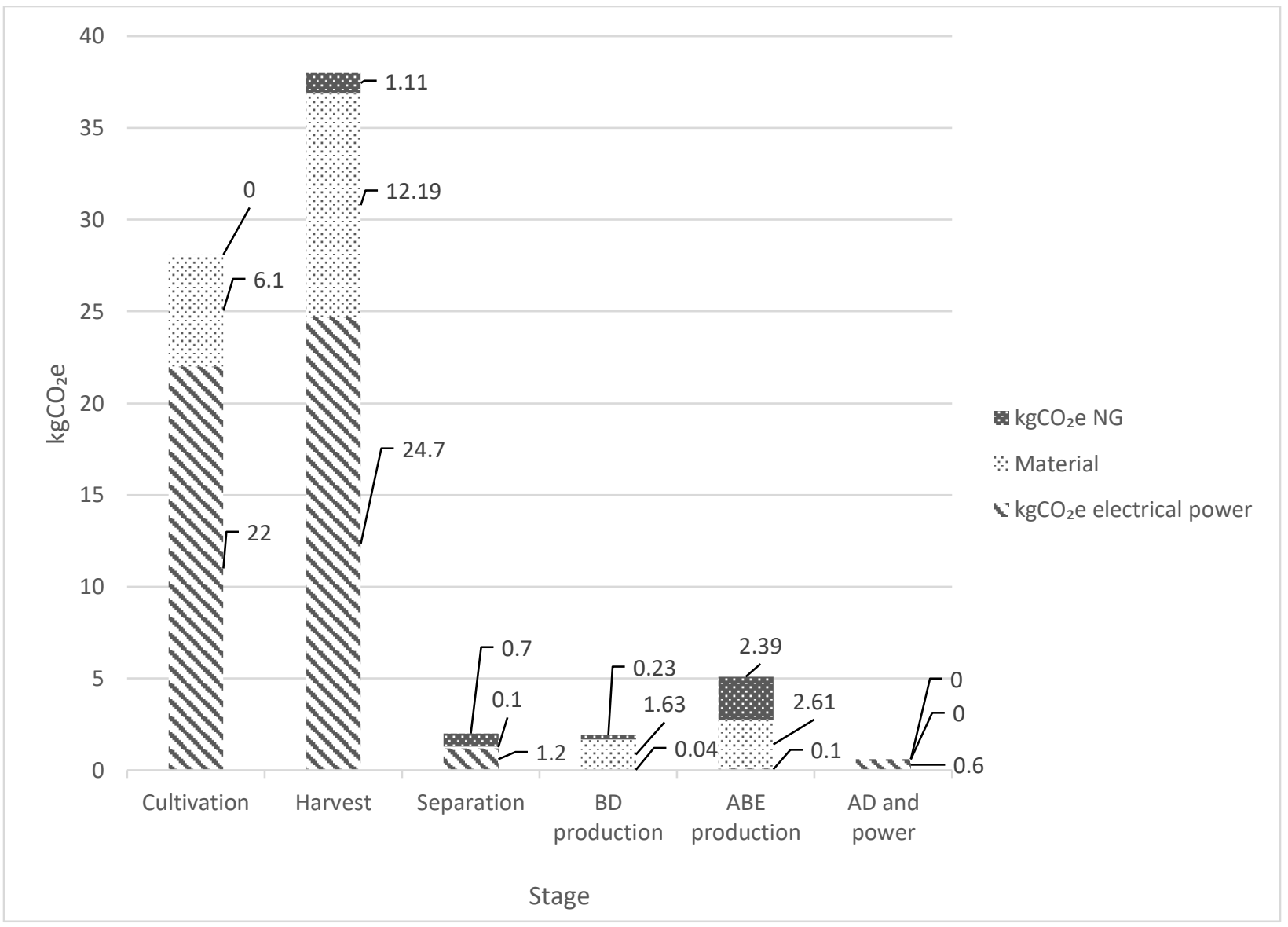

Figure 4.1: GHG impact distribution of NG, material and power per model stage

\subsection{Comparison with other microalgal BD and co-product processes}

All studies mentioned in section 2.5.4 used ponds instead of PBRs. Therefore, it was inevitable that the resulting GHG impact of this study's cultivation stage would be higher than that of other studies. Thirty seven percent of the total GHG impact of this process accrued at this cultivation stage. As mentioned above, other significant trends found in this study include high harvesting impact, low BD production impact and high power impact. Yuan et al. 2015 used a similar process $(\mathrm{AD}$, carbon dioxide re-circulation, water from $\mathrm{AD}$ to support nutrient requirements and co-product biosolids) without ABE production. Yuan et al. (2015) did not provide specific values for energy and GHG impact, however the overall trends were high GHG emissions for cultivation and harvesting and low GHG impact for conversion to $\mathrm{BD}$, which is similar to what this study found. Zaimes \& Khama (2013) did not break down GHG impact into process stages, 
however, the study also found power requirements used the most fossil energy and thus produced the most GHG.

\subsubsection{Argonne National Laboratory study comparison}

Frank et al. (2011) is a microalgal BD GHG LCA study that followed a very similar process to this study. Frank et al. (2011) also provided the most comprehensive supporting information for comparison purposes. Section 2.5.4 of this study outlines Frank et al. (2011)'s parameters. Key differences between Frank et al. (2011) and this study's process include the exclusion of ABE production, the use of a CHP system rather than simply a co-generation facility and the use of a homogenizer to pre-treat the microalgal biomass instead of dry mechanical pre-treatment. Considering the similarity of both processes, each stage of the production process is broken down in the following paragraphs for a more detailed comparison between the two studies.

\section{Cultivation}

Five times more electrical power was required for cultivation in this study than in Frank et al. (2011) (see Appendix E.1.1 for calculations). This is in part because this study used PBRs whereas Frank et al. (2011) used an open pond system. If a reduction of 5 times the amount of electrical power were used in this study, this would correspond to a $62 \%$ reduction in GHG emissions for this stage of the process. Thus, instead of $28.1 \mathrm{kgCO}_{2} \mathrm{e}$ per $100 \mathrm{~km}$ driven in a diesel vehicle, the impact would be around $10.6 \mathrm{kgCO}_{2} \mathrm{e}$. Open pond systems are not an option in Canada if a refinery is to produce year round. Yet, over two thousand PBRs would also take up a considerable amount of space in a populated urban area thus; using PBRs at this scale will likely not be an option either. A reduction in process scale would not alter the operation GHG impacts of the process, however, when including the GHG impact of infrastructure on a per unit biofuel production basis, the GHG impact would increase.

\section{Harvesting}

Frank et al. (2011) used a homogenizer to pre-treat the microalgal biomass before the hexane extraction of lipids. This stage used $365 \mathrm{kWh} / \mathrm{dry}$ ton microalgal biomass. If this process were 
to have used a homogenizer in-lieu of the dryer in this study then it would have saved 3,543 $\mathrm{kWh}$ over the course of an hour (see calculations in Appendix E.1.5). However, because, in this study's case, $\mathrm{NG}$ is used for drying, overall $\mathrm{CO}_{2} \mathrm{e}$ impact is still minimal: $1.1 \mathrm{kgCO}_{2} \mathrm{e}$ compared to the total harvest impact of $37.95 \mathrm{kgCO}_{2} \mathrm{e}$.

There is a substantial difference between the two centrifuge power consumptions of both studies. Frank et al. (2011)'s centrifuge power consumption is based on power required per gram of microalgae, whereas this study's centrifuge power consumption is based on the volume of water that required processing. If this study had used Frank et al. (2011)'s centrifuge power requirement of $3.3 \times 10^{\wedge}-3 \mathrm{kWh} / \mathrm{g}$-microalgae then this study's power requiremnts for this stage's power consumption would have been reduced by $23 \%$ (see calculations in Appendix D.8). However, the total power requirement for Frank et al. (2011)'s final dewatering stage is much less than would be calculated using their own stated centrifugal power requirements. Frank et al. (2011) calculated 3,036 Btu/kg-lipid for the final dewatering stage whereas if $25 \%$ lipid content of microalgae is used, total power requirements for the centrifuge stage for Frank et al. (2011) would be 45,040 Btu/kg-lipid (see calculations in Appendix E.1.3 and E.1.4). This study calculated the equivalent of $41,978 \mathrm{Btu} / \mathrm{kg}$-lipid. This power requirement is less because there is a $35 \%$ lipid content in this study's microalgae.

Regardless, there is a direct correlation between electrical power use and GHG impact, which is apparent in this stage of this study's process. Harvesting is the most electrically intensive stage of this study's process and it accounts for $50 \%$ of the overall GHG impact of this study. If a substantial amount of power could be reduced here, it would reduce the GHG impact of the overall study substantially. The substantial GHG impact associated with harvesting is not unique to this study either. Sander \& Murthy (2010) concluded that $89 \%$ of the energy required to produce the microalgal biomass is required for the harvesting process. It was recognized fairly early on in microalgal biomass cultivation and processing research that the cost of harvesting and dewatering would make or break the economic commercialization of microalgal biofuel production (Balaban et al., 1980).

Considering this study used LCIA electrical power data from the United States of America in GaBi Education, the resulting GHG impact of this study is higher than if Canadian power generation LCIA data had been used. As indicated in section 1.3 of this study, the majority of 
Canada's power generation is hydroelectric and nuclear with only $20 \%$ of electrical power generation directly from fossil resources in general (Natural Resources Canada, 2015b). Ontario specifically uses on average only $6.7 \%$ oil and gas to produce power over the course of a year (see Figure 4.2) and this amount fluctuates (Independent Electricity System Operator, 2018). Ontario produces more electricity using oil and gas in the summer months $(7.8 \%)$ than other times of the year (5.6\%) (Independent Electricity System Operator, 2018).

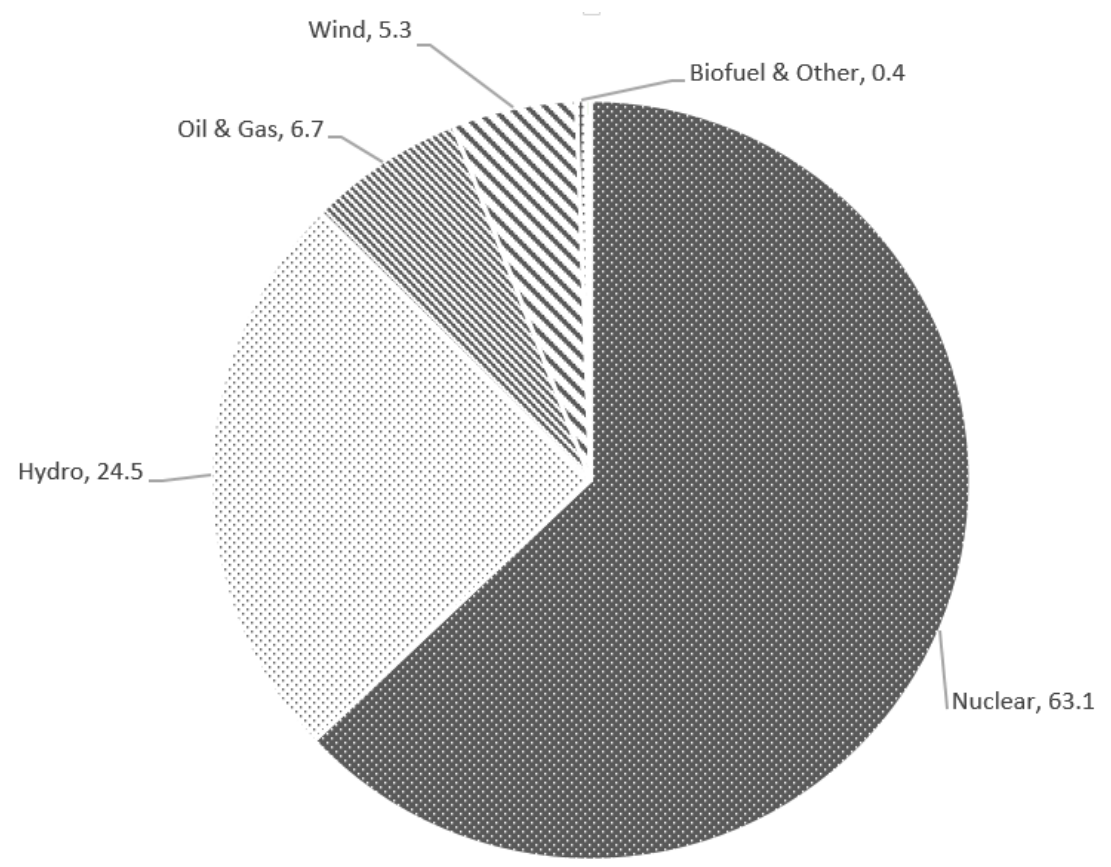

Figure 4.2: Percent Average Ontario Electricity Generation by Source in 2018 adapted from (Independent Electricity System Operator, 2018)

Implemented in Ontario, the estimated GHG impact of this model would range between 31 and $33 \mathrm{kgCO}_{2} \mathrm{e}$ over the course of the year instead of $76 \mathrm{kgCO}_{2} \mathrm{e}$. This change in GHG impact is only by the proportional reduction of fossil resources for the model's operational power use.

\section{Separation}

Frank et al. 2011 only used a 3:1 solvent to oil ratio for lipid separation, which is a lot less than this study's 9:1 ratio, however with the reuse of this solvent and the minimal overall GHG impact of results for this stage, this fact would not have made a significant overall difference. 
Another substantial difference between Frank et al. (2011) and this study is the power production. Frank et al. (2011) require 19,450 Btu/kg-oil for the entire process. The CHP plant generates $14,620 \mathrm{Btu} / \mathrm{kg}$-oil that is used to offset this power requirement. Thus, Frank et al. (2011) generated $75 \%$ of the power requirement on site. In contrast, in this study the process not only requires more power, but also does not produce as much power. This study required the equivalent of $82,980 \mathrm{Btu}$ of electrical power/ kg-oil, which is four times the amount of power required by Frank et al. (2011)'s process on a per kg-oil basis. This study also only produced the electrical power equivalent of $5,460 \mathrm{Btu} / \mathrm{kg}$-oil, which is $7 \%$ of the total power requirement.

One of the reasons for the reduced power production is in part because of the reduced amount of biomass digested in the AD to produce power, as this study uses the lignocellulosic biomass to produce ABE. However, even if all the lignocellulosic biomass were used to generate power in this study, this only adds another $3,858 \mathrm{Btu} / \mathrm{kg}$-oil to the power generated based on the methane and power conversion in this study (see Appendix E.1.8 for calculations). The additional power increases the total generated power of this study's process to 9,426.6 Btu/kg-oil·hr. The difference between this study's power output and Frank et al. (2011)'s study using the same biomass is still substantial. This study's process produces $36 \%$ less power from the same biomass than in Frank et al. (2011) (see Appendix E.1.8 for calculations). There was an attempt made to contact the authors of Frank et al. (2011) to determine discrepancies but the authors were unavailable for comment.

\section{$B D$ and $A B E$ production}

The $\mathrm{BD}$ and $\mathrm{ABE}$ production in this study contributes to $9 \%$ of the overall GHG emissions (7 $\mathrm{kgCO}_{2} \mathrm{e}$ out of $\left.76 \mathrm{kgCO}_{2} \mathrm{e}\right)$. Even though the $\mathrm{BD}$ transesterification process requires supercritical conditions, the resulting GHG impact was minimal. This minimal GHG impact is due to the exothermic chemical reaction taking place as well as the similar heat capacities of the products and reactants. The $\mathrm{ABE}$ process requires more heat, which contributed to $50 \%$ of the total $\mathrm{ABE}$ stage $\mathrm{GHG}$ impact $\left(5 \mathrm{kgCO}_{2} \mathrm{e}\right)$. Frank et al. (2011) indicate a large percent fossil energy use contribution for both the separation and conversion of biomass to fuel process (pg. 42 and 43 of reference); however, are specific amount of energy or $\mathrm{CO}_{2} \mathrm{e}$ for both stages is not 
available or calculable for direct comparison. Larger energy requirements for the sparation stage however, is due to the oil extraction process accommodating a substantial amount of water, whereas this study dries the biomass prior to separation and conversion.

\section{Fertilizer credit}

The fertilizer GHG credit in this study was not substantial enough to influence the overall GHG impact of the process (see Table D.1 in Appendix D.1). Frank et al. (2011) included the $\mathrm{N}_{2} \mathrm{O}$ GHG impact of fertilizing soil. Thus, the GHG impact of using the biosolid fertilizer cancelled out the GHG benefits associated with displacing chemical fertilizer.

\section{Summary}

Overall, the overriding difference between Frank et al. (2011) and this study lies in the cultivation, harvesting and power production processes. In all three of these stages, electrical power use had by far the largest impact on GHG impact. Because of the large GHG impact of electrical power, opting for less material use and more electrical power use for this study produced a higher GHG impact. The BD process stage, in contrast, produced lower GHG impacts. This was expected based on the literature review. The ABE process stage also produced lower GHG impacts than the cultivation and harvesting processes. Note here that there is no discrepancy between the impact factors used to calculate GHG impact in both studies. GaBi uses the IPCC GWP factor to calculate the Climate Change impact category and similar to the GREET model used by Frank et al. (2011).

Although this study's GHG impact is more than Frank et al.'s (2011) study, the model presented in this thesis includes the most significant GHG aspects of the full life cycle of the microalgal BD production process. This model also uses the most developed harvesting and separation processes as well as the most energy efficient operating equipment. Therefore, this model is a realistic representation of the GHG impact of attempting to build and operate such a process today. 


\subsubsection{Biofuel output relative to microalgal biomass input}

A study that greatly influenced the trajectory of this model was the Dong et al. (2016). Dong et al. (2016) completed a similar microalgal BD production process (at lab level) focusing on yield rather than GHG impact. In this study, microalgal biomass is subject to a wet pre-treatment process followed by fermentation to produce ethanol. The ethanol is collected and the biomass feed is subject to transesterification. Dong et al. (2016) found that overall yield (GGE/ton microalgae) was 126 gallons of gasoline equivalent/ton of microalgal biomass (dry weight). This study calculated yield for comparison purposes and found a yield of $186 \mathrm{GGE} /$ ton dry microalgae (see calculations in Appendix E.2). There is no way of knowing how much energy was used to produce the yield in Dong et al. (2016), therefore, there is a strong possibility that more energy is used in this study to produce the increased yield. However, the increased GGE yield using both transesterification and the SSF process is reassuring.

\subsubsection{Net Energy Ratio conclusions}

The original intention was to include total energy requirements in this study's model. However, only primary energy requirements were calculated (i.e., power and NG) for reasons outlined in section 3.1. Primary energy requirements include process energy, but not secondary energy associated with the production of materials required for the process. Even without including secondary energy, total primary process energy for this study, including power reductions from power generated, is $17,400 \mathrm{kWh}$ (summed all energy requirements from Appendix D and found in Figure 4.3 below) compared to 6,513 kWh output (found in Tables A.7 and A.14 as well as Appendix E.2). The resulting overall net energy ratio (NER) is approximately 0.4. NER is typically used to calculate the energy return on an energy product system. NER is the ratio of energy produced by the process divided by the energy used to produce the product energy. In this case, there is much more energy required to produce the product than the energy produced by the process. This NER makes this study unfavourable as there are other microalgal BD studies that produce NERs of upwards of 2 (Clarens et al., 2011). NERs of these studies are discussed in Chapter 5. 


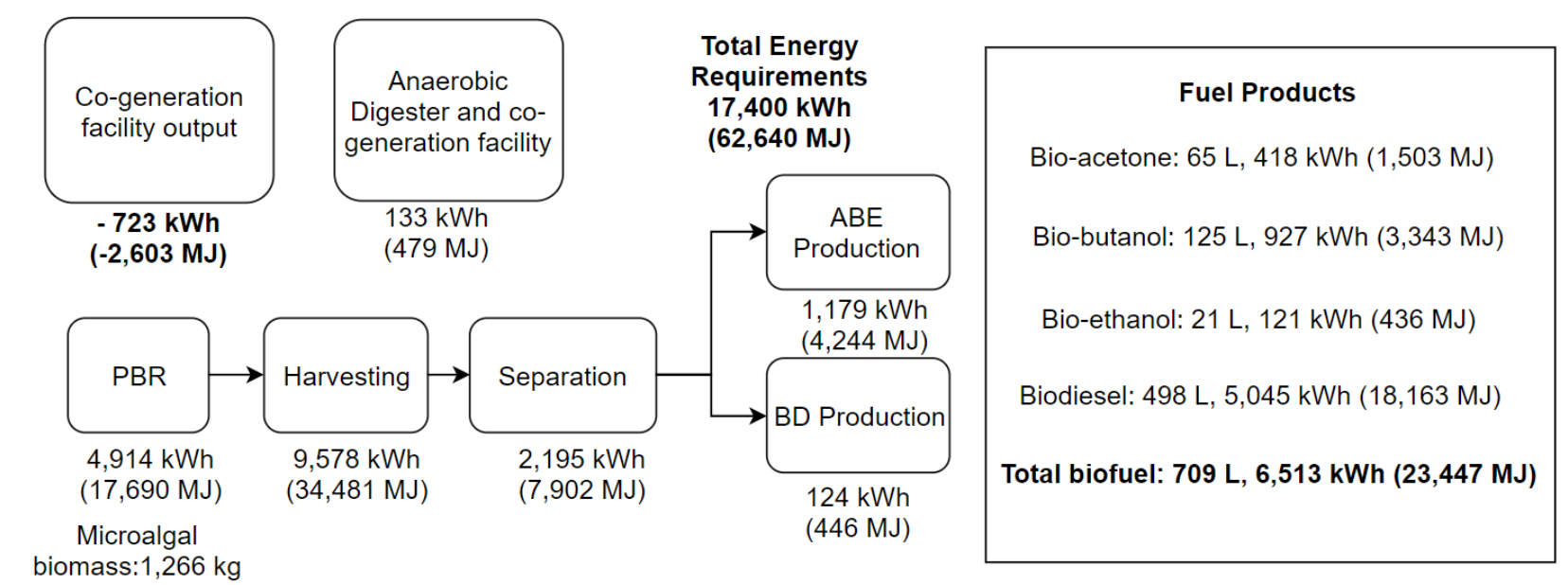

Wastewater: $4.8 \times 10^{\wedge} 6 \mathrm{~L}$

Figure 4.3: Energy requirements (input) and outputs of this study's process on a per hour basis (given an overall study production timeframe of 47 weeks and 10,000,000 $\mathrm{kg}$ of microalgae)

\subsubsection{Feedstock parameter variations}

Without altering this study's process, a variation in lipid, lignocellulosic and protein content would have an effect on the GHG impact of this study's system. As this study broke down GHG impact by stage, it is obvious to see that a lower microalgal lipid content would increase the overall GHG impact of the overall process. The increase in GHG impact is because the ABE process accounts for approximately $4 \%$ more GHG impact overall than the BD process.

As mentioned in section 3.2.3, the microalgal growth rate chosen $(265 \mathrm{mg} / \mathrm{L} \cdot$ day $)$ is conservative and could be improved based on microalgal growth rates found by other studies (e.g. 3.4 $\mathrm{g} / \mathrm{L} \cdot$ day) (de Morais \& Costa, 2007). Increasing the microalgal growth rate per liter of water per day would reduce GHG impact. The reduction in GHG impact would be the result of less processing water circulated and less heat required per microalgal biomass amount during cultivation, harvesting and separation stages. Considering these stages comprise over $50 \%$ of the GHG impact of the overall process, GHG reduction could be significant. The quantity of raw materials (i.e., sodium hydroxide, sulfuric acid and solvents) would remain the same or increase, however, as there would be an increased amount of feedstock that would require processing. Regardless, a substantial increase in microalgal growth rate is unlikely due to the combined use of flue gas and wastewater outlined in section 2.3.4. 


\section{Chapter 5 - Conclusions and Recommendations}

The GHG impact of the microalgal BD processes per $100 \mathrm{VKT}$ of the most recent studies published (Table 2.13 and 2.14) ranges between approximately 3.7 and $85 \mathrm{kgCO}_{2} \mathrm{e}$. Fossil diesel's GHG impact at the same scale is between 17.3 and $23.3 \mathrm{kgCO}_{2} \mathrm{e}$. This study found a 76 $\mathrm{kgCO}_{2}$ e total GHG impact associated with a microalgal BD and $\mathrm{ABE}$ production process measured in $100 \mathrm{VKT}$.

\subsection{Model process adjustment}

Based on this study's results, the following paragraphs outline changes to this study's process that could reduce overall GHG impact.

Considering the difference in power requirements for harvesting (centrifuge) and power generation return between this study and Frank et al. (2011), there is likely some variability in these two areas. Improvements in electrical power requirements for harvesting and electrical power generation would result in a lower GHG impact.

Replacing process NG with stripped methane from AD biogas is not recommended, as this would increase this study's process GHG impact because of the resulting reduction in power generation. At this time, the GHG impact of power is substantially more than the GHG impact of NG, even NG derived from fossil resources. In the future, when power generation relies predominantly on renewable sources, there is potential it would likely be less GHG intensive to recycle on-site methane for heat requirements instead of importing NG from fossil or renewable sources. The energy efficiency of power generation using fuels in gas turbines is also relatively low compared to other forms of energy generation (e.g., hydroelectric, nuclear, boilers) (Cengel $\&$ Boles, 2002), therefore, it would likely be more energy efficient to use the generated biogas for heating purposes instead of power generation.

Instead of using another study's overall energy requirements for tubular PBR cultivation, this study could break down heat and power requirements for cultivation. This breakdown could allow for the incorporation of additional renewable energy sources into the model. Adding solar power to the model for light and power generation purposes will have a positive effect on this 
study's process GHG impact (Subhadra \& Grinson, 2011). Including solar photovoltaic modules would be the first priority considering the large GHG impat of power use. Including solar thermal modules might also decrease the GHG impact of heat energy requirements (Good et al., 2015; Tian \& Zhao, 2013).

As mentioned in section 4.3.1, using wet pre-treatment by homogenization would likely improve the GHG impact of this study's model. Another form of wet-pretreatment that is very energy efficient is ultrasonic treatement. Liang et al. (2012) used ultrasound to pre-treat wet microalgal biomass, but only extracted $49 \%$ of the lipids available in the biomass. This method requires additional research before industrial implementation suitability (Liang et al., 2012).

Other modifications to this study's process could include using wastewater for the lignocellulosic pre-treatment process, thus reducing additional water use (Castro et al., 2015), and using cyanobacteria from fresh water lakes as supplemental feedstock. Including harvested cyanobacteria from fresh water lakes during the summer would increase the biofuel output without having to grow additional biomass. Lake Erie continually experiences nutrient loading and resulting algal blooms because of its centralized location between large urban areas (Watson et al., 2016). However, considering harvesting is the most energy intensive step, this increase in product would likely come with an overall net increase in GHG impact from increased power requirements but a reduced GHG impact on a per-unit fuel basis.

\section{NER}

Although this study focuses on GHG emissions, overall energy use, electrical power or otherwise, is an important aspect of a process because it is directly related to process cost and efficiency. As mentioned in section 4.3.3, other microalgal BD and co-product studies have a larger NER than this study. For example, Clarens et al. (2011)'s study compared the energy return on investement (EROI) of four different microalgal biofuel processes of which two produce BD. EROI is defined the same as NER; the larger the EROI, the better energy return on investment (Colosi, 2012). One of the two BD processes studied by Clarens et al. (2011) produced $\mathrm{BD}$ and bioelectricity from AD, similar to this study's process. The EROI ranged between 1.11 and 1.13. The second of the two BD processes studied by Clarens et al. (2011) produced BD and bioelectricity directly from the combustion of the left over microalgal biomass 
(gasification). The EROI of this later process was 1.99. The main process differences between this study and Clarens et al. (2011) are the use of open ponds and homogenization.

Batan et al. (2010) also uses a form of homogenization for wet processing the microalgal feedstock. The overall NER of Batan et al. (2010)'s mircoalgal BD is 1.1. Batan et al. (2010)'s study was also less GHG intensive than this study's process $\left(4.2 \mathrm{kgCO}_{2} \mathrm{e} / 100 \mathrm{~km} \mathrm{VKT}\right)$ as seen in Table 2.14. Frank et al. (2011) also used homogenization for wet processing and although Frank et al. (2011) did not include a NER, the energy requirements outlined for the homogenization stage are substantially less than this study's combined drying and pulverizing process as seen in section 4.3.1 (Frank et al. 2011). Frank et al. (2011)'s study also predicted a lower GHG impact than this study $\left(10 \mathrm{kgCO}_{2} \mathrm{e} / 100 \mathrm{~km}\right.$ VKT) also seen in Table 2.14.

Based on the results of Frank et al. (2011), Clarens et al. (2011) and Batan et al. (2010), opting for a wet extraction process using homogenization instead of dry processing would likely lower GHG impact and increase the NER of this study's process. It is likely that the GHG and energy savings lie only with the pre-treatment stage and not the follow on processes (i.e., subsequent separation and recycling) for two reasons. First, wet extraction would completely remove the drying NG requirement, but the GHG impact of NG is small. Removing the NG requirement would, however substantially reduce the heat energy requirement. Second, there would be less solvent required downstream if the wet feedstock stream underwent fermentation followed by transesterification as outlined in Dong et al. (2016) (Dong et al. 2016). However, solvent GHG impact is minimal because of solvent recycling. Again, however, there would be less heat energy required to separate the solvent and feedstock mixtures if the process was sequential, similar to Dong et al. (2016). Regardless, there would be more water to heat during the separation of product (i.e., ABE and BD) stages that could outweigh the benefits of reduced solvent and lack of NG drying requirements on GHG impacts and energy consumption (Dong et al. 2016).

To further improve this study's NER, ultrasonic techniques and surfactants during pre-treatment, as well as the use of slower gravity based decanters instead of centrifuges, would improve this model's energy efficiency (Wu et al., 2017). Perfecting these aforementioned processes would potentially slow down the overall process, but would rely more on less energy intensive processes. 


\section{Summary}

Overall, in order to develop a microalgal biofuel production process and system, there is an incentive, along with cost and other parameters, to determine which microalgal biofuel production process is the least GHG intensive to not exacerbate the already blooming GHG levels. All the above modifications would likely align this study's process with the GHG impacts of similar studies yet would not make this process substantially superior from a GHG or energy perspective. In this case, this study's process would not be favoured due to the increased GHG impact and energy efficiency. In the future, it would make sense to focus on the NER for an initial assessment of process superiority and then develop the process to use the the most sustainable power and energy possible.

\subsection{Model development adjustment}

This research found that an LCA type of study supports a cyclical analysis. What this means is a similar study should be set up such that iterations can be performed with different process modifications. This flexibility would make it easier to test a hypothesis after having completed the LCAI stage. For example, using a formulated excel spreadsheet to quickly produce final product values would be beneficial if a future study's objective was to estimate different impacts associated with microalgal biomass with different lipid and lignocellulosic biomass composition. Changing processes, rather than biomass content, would be more labor intensive, but could be facilitated with integrated spreadsheets or a coded program.

This study recommends calculating each process stage (e.g. cultivation, harvesting, BD production etc) GHG impact separately in GaBi. This allows for a better understanding of coproduct allocation and more flexibility of co-product handling.

\subsection{Model purpose adjustment}

This study's co-product allocation would change along with the corresponding GHG impact if this study's purpose had not focused on fuel and instead focused on bio-product development. For example, if this study had considered ABE the primary process bio-chemical products and 
BD a co-product, the GHG impact of all process stages prior to ABE production would be split between the bio-acetone, bio-ethanol and bio-butanol products. The distribution of GHG impact would be based on the amount of each product produced using the mass-based co-product allocation method. The BD production stage GHG impact would also be split between ABE products based on the same ABE output ratio. Furthermore, the GHG benefit of producing BD (renewable fuel) would be credited to the overall process using the substitution allocation method and subsequently the mass based co-product allocation method. Given this shift in study purpose, the resulting GHG impact of the products of the same process but different overall life cycle would be quite different. Given a more energy efficient process and more sustainable forms of power and energy, an LCA of bio-products using a similar process would be a suitable area for future research.

In order to use all the wastewater available from a small WWT plant to grow microalgae, 2,875 40,000 liter PBRs would be required. The space for these PBRs is not practical for set up in an urban setting. A PBR set up in a more rural industrial setting would allow for more of them pending wastewater volume throughput. In this case, microalgae would grow using industrial wastewater rather than municipal wastewater, changing the microalgal growth rate. There is also the additional transport between a rural facility and a biorefinery that would need to be considered in a LCA assessment.

As indicated in section 1.3, this study did not complete a full LCA of microalgal BD and ABE. Because this study focued on operational GHG impact of a microalgal BD and ABE theoretical process, completeing a full LCA that included other impact categories was outside the scope.

\subsection{Overall process conclusions}

The results of this study do, however, provide an understanding of how the addition of an ABE processing step affects the GHG impact of this study's microalgal BD production process. There is additional GHG impact associated with the $\mathrm{ABE}$ process but not to an extent that sets this overall process apart from other microalgal BD and co-product processes. The GHG impact of the ABE processing stage is $6.7 \%$ of the overall process. Given this study's process flow, all other stages of the process, except the separation stage, would have been required in order to produce the other outputs of the process (i.e. BD, fertilizer and energy). Thus, the overall GHG 
impact of including the production of $\mathrm{ABE}$ is minimal. The separation stage only contributed to $2.7 \%$ of the overall GHG impact of the overall process.

It is also apparent based on the results of this study that the source of energy used for the process is rather the problem and not necessarily the carbon footprint. Electrical power requirements make up the majority of the GHG impact of this process (i.e., 66\%) arguably because of the fossil fuel used to produce this electricity (Oreskes, 2018). Rather than continue to explore a diversity of processes to reduce microalgal biofuel production process' GHG emissions below those of a well-established fossil fuel production process, a superior direction would be to reduce fossil use in energy production and elsewhere. This way, a microalgal BD production process might still be carbon intensive, but would be using recycled carbon rather than adding new carbon to the atmosphere. Atmospheric carbon recycling results in a carbon neutral system as carbon has been sequestered from the atmosphere earlier in the life cycle of the process.

Canada is slowly increasing its percent of renewable sources producing electrical power. In 2015, Canadian electrical generation source percentages were $20.2 \%$ fossil $(10.6 \%$ oil/gas \& 9.6\% coal), $15 \%$ nuclear, $58.9 \%$ hydro and $5.9 \%$ renewable (i.e., wind, biomass \& solar) (Natural Resources Canada, 2015b). Based on the Canadian Governments year 2040 predictions, more wind but also more NG will be used to generate electricity (see Figure 5.1). $\mathrm{NG}$ is less $\mathrm{CO}_{2} \mathrm{e}$ intensive for heat generation, as shown in this study in section 4.2.1, but not so for power generation, also demonstrated in this study in section 4.2.1. This reliance on fossil NG for power generation into the future is not acceptable.

The rest of the GHG impact of this study is associated with the carbon footprint of materials. Upon observation of the entire microalgal BD and ABE production process, there are relatively few material inputs involved. Methanol, NG, Dowthern A, hexane, sodium hydroxide and sulfuric acid are the additional materials required in this study's process. There is potential here to derive these materials from biomaterials using biomass energy sources concurrently (Gnansounou \& Pandey, 2017). The import requirement here is the use of biomass or other renewable energy sources to create these materials. Considering the majority of GHG impact comes from the combustion of fuel, the majority of the carbon footprint associated with these materials is from the operational energy requirements used to produce them. 


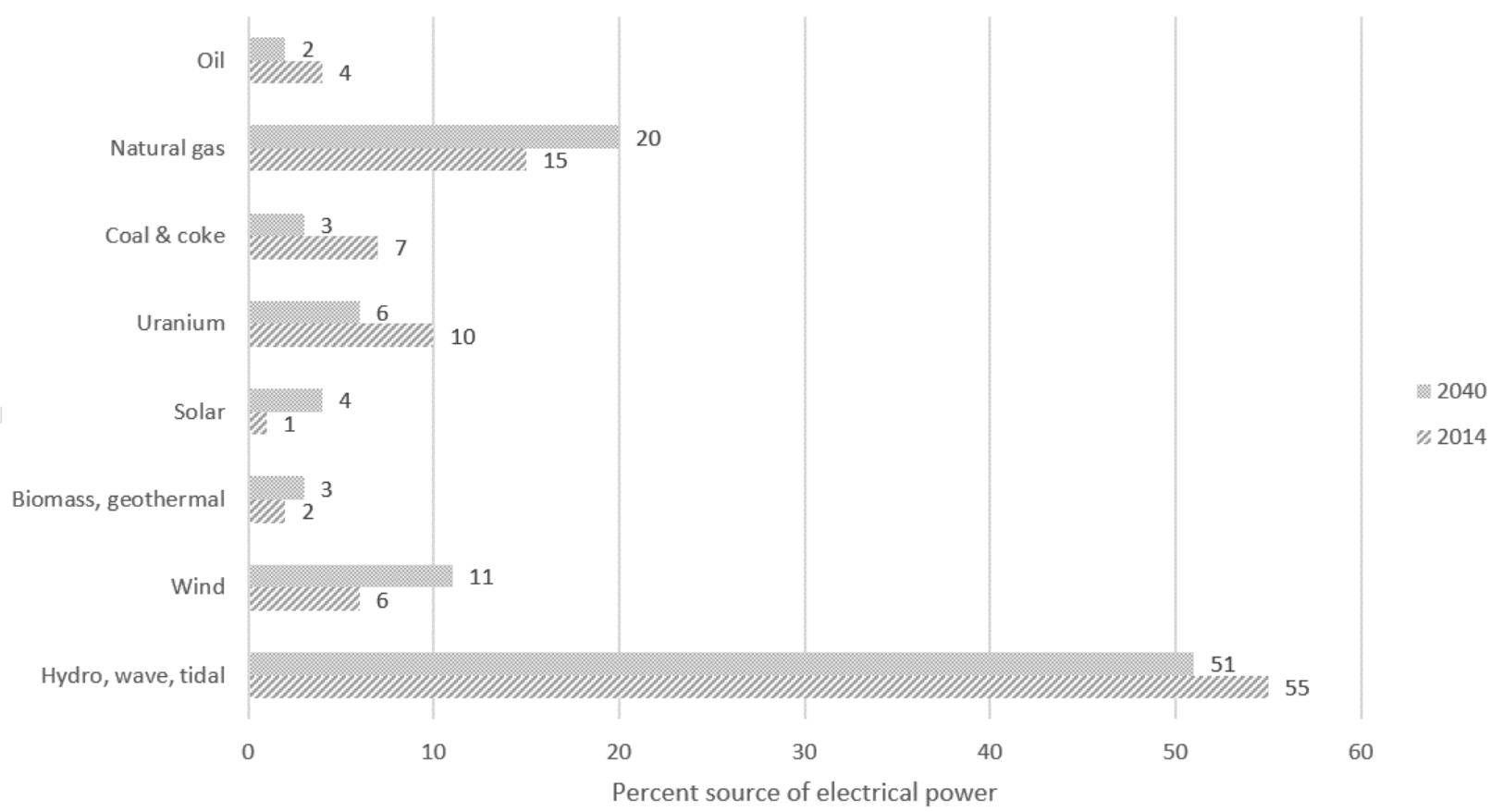

Figure 5.1: Canadian percent electrical power generation sources in 2014 ('14) and predicted for 2040 ('40), Note - read chart and legend from left to right, adapted from (National Energy Board, 2017)

The LCAs that focus on the climate change impact category, similar to this study, are really focused on quantifying the combustion based energy generation required for a process. There are few sources of GHG emissions in engineering processes that are not from the combustion of fuel. As regions power and heat energy generation sources shift to renewable (e.g. hydro, solar, wind, wave, biomass), the measure of GHG impact in LCAs will decrease.

\subsection{Anthropogenic system changes (The biorefinery concept and distribution system)}

There is undoubtedly a need to integrate systems. Similar to the "grid" concept outlined by Harvard's Ms. Oreskes in her Vancouver lecture, there is a need to integrate wastewater treatment, flue gas sequestration and the production of liquid fuels (Oreskes, 2018). To put this into context, the Barrie wastewater treatment plant produces and cleans $5.89 \times 10^{\wedge} 7 \mathrm{~L}$ of wastewater per day (City of Barrie, 2004). This study’s process requires $1.15 \times 10^{\wedge} 8 \mathrm{~L}$ of wastewater per day. Therefore, a larger wastewater treatment plant is required to produce 10,000 
tonnes of microalgae per year (or approximately 5.7 million liters of total biofuel). When compared to other BD production plants operating in Canada today, the fuel product output of 5.7 million liters is relatively small.

If all the wastewater in the United States (122,439 million litres of wastewater/day) were used to produce fuel, with a $90 \%$ removal of limiting nutrient from wastewater, a 10\% BD yield and a 9 months/year operation, the overall process would produce roughly 6.5 million liters of fuel/day (Christenson \& Sims, 2011). The United States uses 1,430 million liters/day of transport fuel. Producing fuel with wastewater amounts for $0.5 \%$ of the total transport fuel required by the US in 2011. Even contributing to such a small amount of a country's fuel requirements, Pittman et al. 2012 and Lundquist et al. 2010 insist the algal growth for biofuel production in consort with wastewater treatment is the only commercial hope for algal biofuel production (Lundquist et al., 2010; Pittman et al., 2011). Considering if it were not for the use of wastewater, 50\% of the GHG emissions associated with algal cultivation would come from chemical fertilizers, this assertion makes sense (Wiley et al., 2011). The United States of America NREL also insists that there is no better way to learn effective integration of biofuel production and wastewater treatment process techniques than to do it on a small scale (Shelef et al., 1984).

Furthermore, there is a need to sequester flue gas. However, without the reduction of fossil fuel use, sequestering this carbon using microalgae and emitting it back into the atmosphere upon combustion, if the algae is used as a fuel source, nullifies any sequestering effects. Using microalgae that has sequestered flue gas to produce other products instead of fuel might help prevent the cycling of fossil carbon back into the air. Sourcing such microalgae for a human food source would be infeasible for health reasons, but there might be a bio-material process pathway that could suffice.

There is adequate technology to produce microalgal biofuel. $\mathrm{BD}$ and alcohol produced by the transesterification of plant based oils and the fermentation of plant based carbohydrates respectively were readily used to produce fuel from biomass at the beginning of the twentieth century (Arbor, 1986). However, since the early twentieth century, society has changed such that there are more people in concentrated areas requiring food and energy. As micro and macroalgae have the potential to be a very important food source, and are already used as supplements (Kelly, Ikonomou, Blair, Morin, \& Gobas, 2007; Suganya et al., 2016), microalgae 
use for transport fuel purposes might be limited. Also considering livestock production is one of the leading producers of GHG, potentially more so than the transportation industry (Agriculture \& Agri-Food Canada, 2018; Andersen \& Kuhn, 2014; McMichael et al. 2007), society will inevitably need to reduce its reliance on livestock as a food source. People will thus need to rely more on lower trophic level organisms for nutrition, including microalgae.

Producing copious quantities of microalgal biofuel initially or even in the future does not have to and should not have to be the main objective of an integrated microalgal biofuel production system, or any anthropogenic production system. The argument that a negative economic impact will result from an increase in biofuel development, and the decarbonisation of the world's energy system, without the support of an economy of scale, is still used today to prevent changes to the transport energy production system status quo. However, there are multiple sources that negate this argument and propose that economic variability is controlled by large and small value based decisions by influential decision makers (Arbor, 1986; Gibney, 2018; Harari, 2014; Oreskes, 2018; Weaver, 2017). Government initiatives have contributed to successful increases in biofuel use, thus making it relatively clear that the only way to increase the use of renewables is to have some kind of overarching accountable control over the economy. Similarly, overarching energy goals should foster the diversification of energy and fuel sources. There should be an aversion to creating a single sourced, non-redundant fuel and chemical industry as was done with fossil resources. As such, it is likely that in the future, liquid fuels will be reserved for certain types of transport in potentially more remote locations such that much less liquid fuel will be required overall. 


\section{Appendices}

Appendix A - Input/Output (IO) process parameters and calculations

\section{A.1 IO for PBR}

Table A.1: Cultivation input/output table

\begin{tabular}{|c|c|c|c|c|c|c|}
\hline Stage & & & Input amount & & & Output amount \\
\hline $\begin{array}{l}\text { PBR } \\
\text { (PBR 01) }\end{array}$ & $\begin{array}{l}1 \\
2 \\
3 \\
4 \\
5 \\
6 \\
7 \\
8\end{array}$ & $\begin{array}{l}\text { Microalgae } \\
\text { Glycerol (nutrients) } \\
\text { Sunlight } \\
\text { Nitrogen } \\
\text { Phosphorus } \\
\mathrm{CO}_{2} \\
\text { Wastewater } \\
\text { Energy requirements }\end{array}$ & $\begin{array}{l}0 \\
\mathbf{4 1 1 , 7 9 2} \mathbf{~ k g} / \text { year } \\
\text { Given } \\
\mathbf{6 9 . 5 2} \mathrm{kg} \mathrm{N} / \mathrm{hr} \\
3.91 \mathrm{~kg} \mathrm{P} / \mathrm{hr} \\
\mathbf{1 , 6 4 3} \mathrm{kg} \mathrm{CO} / \mathrm{hr} \\
1.15 \times 10^{\wedge} 8 \mathrm{~L} / \text { day } \\
\mathbf{1 5 3} \mathbf{G W h}(\text { over } 1 \text { year) }\end{array}$ & $\begin{array}{l}1 \\
2 \\
3\end{array}$ & $\begin{array}{l}\text { Algae } \\
\text { Wastewater } \\
\text { Oxygen }\end{array}$ & $\begin{array}{l}10,000,000 \mathrm{~kg} / \text { year } \\
\mathbf{1 . 1 5 x 1 0}^{\wedge} \mathbf{~ L / d a y} \\
\text { Purged (not used) }\end{array}$ \\
\hline
\end{tabular}

\section{A.1.1 PBR 01 - Tubular photobioreactor (input \#2, 4, 5, 6, 7, 8, output \#1)}

Details/Assumptions:

- Tubular PBR

- Growth of $C$. vulgaris ranges in PBR using different types of wastewater and nutrients is between $85 \mathrm{mg} / \mathrm{L} \cdot \mathrm{day}$ to $3.4 \mathrm{~g} / \mathrm{L} \cdot \mathrm{day}$ (de Morais \& Costa, 2007). This study chose Ma 2016 as a reference value to be conservative $265 \mathrm{mg} / \mathrm{L} \cdot \mathrm{day}$ (Ma, 2016).

- Circulation pump: 0.5 HP/PBR (Min et al., 2014)

- This study did not include the energy requied to initially grow the microalgae in each of the PBRs, although this would take approximately 7 days and additional nutrients. 
- This study assumed that 5 weeks of the year the plant will be down for maintenance/holidays (plant operates 47 weeks/year)

- Phosphorus, nitrogen and carbon nutrient values optimal for algal growth: C:N:P ratio of 100:11:1 (Chisti, 2007)

- $\quad \mathrm{CO}(0.48) \mathrm{H}(1.83) \mathrm{N}(0.11) \mathrm{P}(0.01)($ Chisti, 2007)

- Molecular weight of microalgae: $23.36 \mathrm{~g} / \mathrm{mol}$ microalgae (based on Chisti 2007 chemical formula)

- Molecular weight of FAME: 0.292kg/mol (Agarwal \& Das, 2001)

- Organic carbon content of biosolids is between 20 and 50\% (Torri et al. 2014)

- Biosolids are removed from the system for fertilizer use, however, the supernatant, which contains more than enough nutrients for the PBR is used to suppliment nutrients where required.

- This study uses typical wastewater total nitrogen and total phosphorus levels: $141 \mathrm{mg} / \mathrm{L}$ and $178 \mathrm{mg} / \mathrm{L}$ respectively (Ma, 2016)

- Other microalgal cultivation with municipal wastewater had $\mathrm{N}$ and P concentrations vary between 120.6 - $530 \mathrm{mg} / \mathrm{L}$ of total phosphorus and 128.6 - $290 \mathrm{mg} / \mathrm{L}$ of total nitrogen (Kong et al., 2010; Min et al., 2011)

- Medium lipid microalgal biomass (35\% lipids) have approximately $35 \% \mathrm{P}$ in lipids and $65 \% \mathrm{P}$ in nucleic acids as well as $88 \%$ $\mathrm{N}$ in proteins and $11 \% \mathrm{~N}$ in nucleic acids (Williams \& Laurens, 2010). In the case presented in GREET 2017, virtually no P is found in the proteins, carbohydrates and lipids (United States of America Argonne National Laboratory, 2017; Williams \& Laurens, 2010). Therefore, as this process is extracting most of the lipids and lignocellulosic (carbohydrate) biomass, this leaves almost all the $\mathrm{N}$ in the left over biomass and the majority of the $\mathrm{P}$. Therefore, considering $\mathrm{N} \& \mathrm{P}$ are suitable for continued recycling within system.

- This study assumes full $\mathrm{N}$ and $\mathrm{P}$ recovery and circulation. 
Table A.2: PBR nutrient sources

\begin{tabular}{|l|l|}
\hline Nutrient & Source \\
\hline Carbon & Flue from co-gen facility $\left(\mathrm{CO}_{2}\right.$ and flue $)$ \\
\hline Carbon & Glycerol \\
\hline Carbon & Anaerobic digester supernatant \\
\hline Carbon & Wastewater \\
\hline Carbon & Flue gas from industry to ensure nutrient balance and sequestration \\
\hline Carbon & Fermenter (ABE process) \\
\hline Nitrogen & Anaerobic digester supernatant \\
\hline Nitrogen & Wastewater \\
\hline Phosphorus & Anaerobic digester supernatant \\
\hline Phosphorus & Wastewater \\
\hline
\end{tabular}

\section{Calculations:}

Input \#7 - Wastewater requirement

$265 \mathrm{mg} / \mathrm{L} \cdot$ day $=0.265 \mathrm{~g} / \mathrm{L} \cdot$ day $=2.65 \times 10^{\wedge}-4 \mathrm{~kg} / \mathrm{L} \cdot$ day

$2.65 \times 10^{\wedge}-4 \mathrm{~kg} / \mathrm{L} \cdot$ day $\cdot 0.001$ tonnes $/ \mathrm{kg}=2.65 \times 10^{\wedge}-7$ tonnes $/ \mathrm{L} \cdot$ day

10,000 tonnes/year / 47 weeks/year / 7 days/week $=30.4$ tonnes/day

30.4 tonnes/day $/ 2.65 \times 10^{\wedge}-7$ tonnes $/ \mathrm{L} \cdot$ day $=1.15 \times 10^{\wedge} 8 \mathrm{~L}$ water (required per day)

\section{Input \#8 - Energy requirements for PBR operation}

Details/Assumptions:

- 153 GWh (over the course of the year) (Harun et al., 2011). This includes:

○ PBR circulation

○ Solar requirements 
○ Cooling water pumping as required

- Based on a production of $50 \mathrm{kt} /$ year (5x that of which is produced here):

Calculations:

$153 \mathrm{Gwh}=153 \cdot 3,600,000 \mathrm{MJ}=550,800,000 \mathrm{MJ}($ per year $)=69,757 \mathrm{MJ} / \mathrm{hr}$

For this process estimate:

$69,757 \mathrm{MJ} / \mathrm{hr} / 5=13,951 \mathrm{MJ} / \mathrm{hr}$

Input \#2, 6 - Carbon calculations $\left(\mathrm{CO}_{2}\right.$ and glycerol)

Total amount of carbon in microalgae produced:

$10,000,000 \mathrm{~kg}$ microalgae/year/47 week/year/7days a week $/ 24$ hours $/$ day $=1.27 \times 10^{\wedge} 3 \mathrm{~kg}$ of microalgae $/ \mathrm{hr}$

$1.27 \times 10^{\wedge} 3 \mathrm{~kg}$ microalgae $/ \mathrm{hr} / 0.02336 \mathrm{~kg} / \mathrm{mol}=54,215 \mathrm{~mol} / \mathrm{hr}$

$54,215 \mathrm{~mol} / \mathrm{hr} \cdot 0.012 \mathrm{~kg} / \mathrm{mol}=650.6 \mathrm{~kg} \mathrm{C} / \mathrm{hr}$

Total amount of carbon in products (ABE, BD, fertilizer):

Carbon in FAME:

$430 \mathrm{~kg} / \mathrm{hr} / 0.292 \mathrm{~kg} / \mathrm{mol}=1,473 \mathrm{~mol} / \mathrm{hr}$

$17 \mathrm{~mol} \mathrm{C} / \mathrm{mol}$ FAME

$17 \mathrm{~mol}$ of $\mathrm{C} \cdot 1,473 \mathrm{~mol} \mathrm{FAME} / \mathrm{hr}=25,034 \mathrm{~mol} \mathrm{C} \cdot 0.012 \mathrm{~kg} / \mathrm{mol} \mathrm{C}=300 \mathrm{~kg} \mathrm{C}$

Carbon in Acetone: 
$50.76 \mathrm{~kg} / \mathrm{hr} / 0.058 \mathrm{~kg} / \mathrm{mol}=875.17 \mathrm{~mol} / \mathrm{hr}$

$3 \mathrm{~mol} \mathrm{C} / \mathrm{mol}$ Acetone

$3 \mathrm{~mol}$ of C $875.17 \mathrm{~mol}$ acetone $/ \mathrm{hr}=2,626 \mathrm{molC} / \mathrm{hr} \cdot 0.012 \mathrm{~kg} / \mathrm{mol} \mathrm{C}=31.5 \mathrm{~kg} \mathrm{C}$

Carbon in Butanol:

$101.52 \mathrm{~kg} / \mathrm{hr} / 0.074 \mathrm{~kg} / \mathrm{mol}=1372 \mathrm{molC} / \mathrm{hr}$

$4 \mathrm{~mol}$ of $\mathrm{C} / \mathrm{mol}$ butanol

$4 \mathrm{~mol}$ of C $\cdot 1,372 \mathrm{~mol} \mathrm{C} / \mathrm{hr}=5,488 \mathrm{molC} / \mathrm{hr} \cdot 0.012 \mathrm{~kg} / \mathrm{mol} \mathrm{C}=65.86 \mathrm{~kg} \mathrm{C}$

Carbon in Ethanol:

$16.92 \mathrm{~kg} / \mathrm{hr} / 0.046 \mathrm{~kg} / \mathrm{mol}=368 \mathrm{~mol}$ ethanol $/ \mathrm{hr}$

$2 \mathrm{~mol} \mathrm{C} / \mathrm{mol}$ ethanol

$2 \mathrm{~mol} \mathrm{C} \cdot 368 \mathrm{~mol} \mathrm{ethanol} / \mathrm{hr}=735.65 \mathrm{~mol} \mathrm{C} / \mathrm{hr} \cdot 0.012 \mathrm{~kg} / \mathrm{mol} \mathrm{C}=8.83 \mathrm{~kg} \mathrm{C}$

Total carbon lost with products (bio-acetone, bio-butanol, bio-ethanol, FAME)

$300 \mathrm{~kg} \mathrm{C}$ in FAME $+31.5 \mathrm{~kg} \mathrm{C}$ in bio-acetone $+65.86 \mathrm{~kg} \mathrm{C}$ in bio-butanol $+8.83 \mathrm{~kg} \mathrm{C}$ in bio-ethanol $=406.19 \mathrm{~kg} \mathrm{C}$ in $\mathrm{product} / \mathrm{hr}$

Organic carbon content of biosolids (20\%)

$290.9 \mathrm{~kg}$ biosolid $\cdot 0.20=42 \mathrm{~kg}$ carbon $/ \mathrm{hr}$

Total carbon recycled in the system: 
$650.6-406 \mathrm{~kg} \mathrm{C} / \mathrm{hr}-42 \mathrm{~kg} \mathrm{C} / \mathrm{hr}=202 \mathrm{~kg} \mathrm{C} / \mathrm{hr}$ or $\mathbf{1 . 5 9 x 1 0}{ }^{\wedge} \mathbf{6} \mathbf{~ k g ~ C / y e a r}$ (carbon from glycerol, $\mathrm{CO}_{2}$ from fermenter and flue gas after burning biogas in co-gen)

Mass Balance confirmation (calculating the amount of carbon recycled in recycled glycerol, $\mathrm{CO}_{2}$ from fermenter and flue gas after burning biogas in co-gen)

$\mathrm{CO}_{2}$ purged from airlift bioreactor $\left(1.26 \times 10^{\wedge} 6 \mathrm{~kg} / \mathrm{year} *\right)+\mathrm{CO}_{2}$ produced by $\mathrm{AD}$ of proteins/lignocellulosic/lipid biomass $\left(2.02 \times 10^{\wedge} 6 \mathrm{~kg} \mathrm{CO}_{2}+3.78 \times 10^{\wedge} 5 \mathrm{~kg} \mathrm{CO}_{2}+7.14 \times 10^{\wedge} 5 \mathrm{~kg} \mathrm{CO}_{2} /\right.$ year $)=1.26 \times 10^{\wedge} 6+3.1 \times 10^{\wedge} 6 \mathrm{~kg} /$ year $=4.37 \times 10^{\wedge} 6 \mathrm{~kg} \mathrm{CO}_{2} /$ year

In terms of carbon:

$4.37 \times 10^{\wedge} 6 \mathrm{kgCO}_{2} /$ year $/ 0.044 \mathrm{kgCO}_{2} / \mathrm{mol}=9.93 \times 10^{\wedge} 7 \mathrm{molCO}_{2} /$ year

Given molar ratio $\mathrm{CO}_{2}: \mathrm{C}$ is $1: 1,9.93 \times 10^{\wedge} 7 \mathrm{molCO}_{2} /$ year $=9.93 \times 10^{\wedge} 7 \mathrm{molC} /$ year

$9.93 \times 10^{\wedge} 7 \mathrm{molC} /$ year* $0.012 \mathrm{~kg} / \mathrm{molC}=1.19 \times 10^{\wedge} 6 \mathrm{kgC} /$ year

*see IO for ABE for calculations

Recycled carbon from glycerol production:

Reaction: $\mathrm{TG}+3 \mathrm{MeOH} \leftrightarrow \mathrm{GL}+\mathrm{FAME}$

$429.53 \mathrm{~kg}$ FAME$/ \mathrm{hr} / 0.2965 \mathrm{~kg} / \mathrm{mol}=1,448.67 \mathrm{~mol} \mathrm{FAME} / \mathrm{hr}$

1,448.67 mol glycerol/hr

Each mol of glycerol provides $3 \mathrm{~mol}$ of carbon (Glycerol $\mathrm{C}_{3} \mathrm{H}_{8} \mathrm{O}_{3}$ )

$1,448.67 \mathrm{~mol}$ glycerol $/ \mathrm{hr} \cdot 3=4,346 \mathrm{~mol} \mathrm{C} / \mathrm{hr}$ 
$4,346 \mathrm{~mol} \mathrm{C} / \mathrm{hr} \cdot 0.012 \mathrm{~kg} / \mathrm{mol}=52 \mathrm{~kg} \mathrm{C} / \mathrm{hr}$

$52.15 \mathrm{~kg} \mathrm{C} / \mathrm{hr} \cdot 24 \mathrm{hr} /$ day $\cdot 7$ day/week 47 weeks $/$ year $=411,792.5 \mathrm{~kg}$ C/year

Mass balance calculation total carbon recycled: $1.19 \mathrm{x} 10^{\wedge} 6 \mathrm{kgC} / \mathrm{year}$ from fermenters and $\mathrm{AD}+411,792.5 \mathrm{~kg} \mathrm{C} / \mathrm{year}$ from glycerol $=$ $1.6 \times 10^{\wedge} 6 \mathrm{kgC} /$ year

$$
1.6 \times 10^{\wedge} 6 \mathrm{kgC} / \text { year 1.59x } 10^{\wedge} 6 \mathrm{kgC} / \text { year }
$$

Total $\mathrm{CO}_{2}$ required (due to loss from products/fertilizer):

$406 \mathrm{~kg} \mathrm{C}$ lost in biofuel products $/ \mathrm{hr}+42 \mathrm{~kg} \mathrm{C}$ lost in fertilizer $/ \mathrm{hr}=448 \mathrm{~kg} \mathrm{C} / \mathrm{hr}$

$448 \mathrm{~kg} \mathrm{C} / \mathrm{hr} / 0.012 \mathrm{~kg} / \mathrm{mol} \mathrm{C}=37,333 \mathrm{~mol} \mathrm{C} \cdot 0.044 \mathrm{~kg} / \mathrm{mol} \mathrm{CO}_{2}=1,643 \mathrm{~kg} \mathrm{CO} / \mathrm{hr}$

Input \#4, 5 - Nitrogen and Phosphorous

Total amount of $\mathrm{N}$ and $\mathrm{P}$ required to satisfy $\mathrm{C}: \mathrm{N}: \mathrm{P}$ of 100:11:1

$650.6 \mathrm{~kg} \mathrm{C} / 100=x \mathrm{~kg} \mathrm{~N} / 11, \mathrm{x}=\mathbf{7 1 . 5 7} \mathrm{kg}$ of $\mathrm{N} / \mathrm{hr}$

$650.6 \mathrm{~kg} \mathrm{C} / 100=x \mathrm{~kg} \mathrm{P} / 1, \mathrm{x}=\mathbf{6 . 5} \mathbf{~ k g ~ o f ~ P / h r}$

Total amount of $\mathrm{N}$ and $\mathrm{P}$ in wastewater using $\mathrm{Ma}(2016)$ values:

$0.178 \mathrm{~g} \mathrm{P} / \mathrm{L} \cdot 1.15 \times 10^{\wedge} 8 \mathrm{~L} /$ year $=2.05 \times 10^{\wedge} 7 \mathrm{~g} /$ year $=2.59 \mathrm{~kg} \mathrm{P} / \mathbf{h r}$

$0.141 \mathrm{~g} \mathrm{~N} / \mathrm{L} \cdot 1.15 \times 10^{\wedge} 8 \mathrm{~L} /$ year $=1.62 \times 10^{\wedge} 7 \mathrm{~g} /$ year $=\mathbf{2 . 0 5} \mathbf{~ k g ~ N} / \mathbf{h r}$

Nitrogen and Phosphorus additional requirements: 


\section{$71.57 \mathrm{~kg}$ of N/hr $-2.05 \mathrm{~kg} \mathrm{~N} / \mathrm{hr}=\mathbf{6 9 . 5 2} \mathrm{kg} \mathrm{N} / \mathrm{hr}$}

$6.5 \mathrm{~kg}$ of $\mathrm{P} / \mathrm{hr}-2.59 \mathrm{~kg} \mathrm{P} / \mathrm{hr}=3.91 \mathrm{~kg} \mathrm{~N} / \mathrm{hr}$

$\mathrm{N}$ and $\mathrm{P}$ in wastewater will be recycled through the digestion process (biosolid supernatant returned to PBR)

Approximate time it will require to build up enough $\mathrm{N}$ and $\mathrm{P}$ to supplement the levels of $\mathrm{N}$ and $\mathrm{P}$ required:

$71 / 2.05=34$ - it will only take 34 hours for enough $\mathrm{N}$ (theoretically) to be retained in the system for adequate nutrient maintenance

$6.5 / 2.59=3$ - it will only take 3 hours for enough $\mathrm{P}$ (theoretically) to be retained in the system for adequate nutrient maintenance

Therefore, biosolids will exit the process as fertilizer for elsewhere. GHG credits are calculated in IO for AD section

A.2 IO for Harvesting

Table A.3: Harvesting input/output table

\begin{tabular}{|c|c|c|c|c|c|c|}
\hline Stage & & Inputs & Input amount & & Outputs & Output amount \\
\hline $\begin{array}{l}\text { Flocculation } \\
\text { and } \\
\text { Floatation } \\
\text { (FLC 01) }\end{array}$ & $\begin{array}{l}1 \\
2 \\
3 \\
4 \\
5\end{array}$ & $\begin{array}{l}\text { Wastewater } \\
\text { Algae } \\
\mathrm{NaOH} \\
\text { Sulfuric acid } \\
\text { Power }\end{array}$ & $\begin{array}{l}1.15 \times 10^{\wedge} 8 \mathrm{~L} / \text { day } \\
\mathbf{1 0 , 0 0 0 , 0 0 0} \mathbf{~ k g} / \text { year } \\
7,564,368 \mathrm{~kg} / \text { year } \\
\mathbf{1 , 1 7 6 . 8 ~} \mathbf{~ k g} / \mathbf{h r} \\
72 \mathrm{kWh}(1 \text { hour})\end{array}$ & $\begin{array}{l}1 \\
2\end{array}$ & $\begin{array}{l}\text { Wastewater } \\
\text { Algae }\end{array}$ & $\begin{array}{l}1.09 \times 10^{\wedge} 8 \mathrm{~L} / \text { day } \\
\mathbf{1 0 , 0 0 0 , 0 0 0} \mathbf{~ k g} / \text { year }\end{array}$ \\
\hline $\begin{array}{l}\text { Centrifuge } \\
\text { (CEN 01) }\end{array}$ & $\begin{array}{l}6 \\
7 \\
8\end{array}$ & $\begin{array}{l}\text { Wastewater } \\
\text { Algae } \\
\text { Energy }\end{array}$ & $\begin{array}{l}1.09 \times 10^{\wedge} 8 \mathrm{~L} / \text { day } \\
\mathbf{1 0 , 0 0 0 , 0 0 0} \mathbf{~ k g} / \text { year } \\
5,450 \mathrm{kWh}(1 \text { hour })\end{array}$ & $\begin{array}{l}3 \\
4\end{array}$ & $\begin{array}{l}\text { Wastewater } \\
\text { Algae }\end{array}$ & $\begin{array}{l}7.63 \times 10^{\wedge} 7 \mathrm{~L} / \text { day } \\
10,000,000 \mathrm{~kg} / \text { year }\end{array}$ \\
\hline $\begin{array}{l}\text { Flash Dryer } \\
\text { (DRY 01) }\end{array}$ & $\begin{array}{l}9 \\
10\end{array}$ & $\begin{array}{l}\text { Wastewater } \\
\text { Algae }\end{array}$ & $\begin{array}{l}7.63 \times 10^{\wedge} 7 \mathrm{~L} / \text { day } \\
\mathbf{1 0 , 0 0 0 , 0 0 0} \mathbf{~ k g} / \text { year }\end{array}$ & 5 & Algae & $10,000,000 \mathrm{~kg} /$ year \\
\hline
\end{tabular}




\section{A.2.1 FLC 01 - Flocculation (input \#3, 4, 5, output \#1)}

Details/Assumptions:

- Flocculation energy required is $0.015 \mathrm{kWh} / \mathrm{m}^{3}$ (Vandamme, 2013)

- Assuming density of $\mathrm{NaOH}$ and algae is negligible here

- Final slurry \% total solids: 3-5\% (Vandamme, 2013; Wiley et al., 2011) = loss of 5\% water

- $5 \mathrm{mM}$ of $\mathrm{NaOH}$ required (Yang et al., 2016)

- Molar mass $\mathrm{NaOH}: 40 \mathrm{~g} / \mathrm{mol}$

- Assuming no algae loss

Calculations:

Input \#3 - sodium hydroxide

$5 \mathrm{mmol} \mathrm{NaOH} / \mathrm{L} \cdot 1.15 \times 10^{\wedge} 8 \mathrm{~L} /$ day $=5.75 \times 10^{\wedge} 8 \mathrm{mmol} \mathrm{NaOH} /$ day

$5.75 \times 10^{\wedge} 8 \mathrm{mmol} \mathrm{NaOH} /$ day $\cdot 0.04 \mathrm{~g} / \mathrm{mmol}=2.3 \times 10^{\wedge} 7 \mathrm{~g} /$ day

$2.3 \times 10^{\wedge} 7 \mathrm{~g} /$ day $\cdot 1$ day $/ 24$ hours $=9.58 \times 10^{\wedge} 5 \mathrm{~g} / \mathrm{hr}=958 \mathrm{~kg} / \mathrm{hr}=7,564,368 \mathrm{~kg} /$ year

Input \#5 - power

$0.015 \mathrm{kWh} / \mathrm{m}^{3} \cdot 115,000 \mathrm{~m}^{3} /$ day $($ wastewater $)=1,725 \mathrm{kWh} /$ day

$72 \mathrm{kWh}$ (over an hour)

Output \#1 - wastewater 
$1.15 \times 10^{\wedge} 8 \mathrm{~L}$ wastewater/day $-\left(0.05 \cdot 1.15 \times 10^{\wedge} 8 \mathrm{~L}\right.$ wastewater $/$ day $)=1.09 \times 10^{\wedge} 8 \mathrm{~L} /$ day

Additional wastewater created because of the neutralization of flocculant:

$12,008.3 \mathrm{~mol} \mathrm{H}_{2} \mathrm{SO}_{4} / \mathrm{hr}$ and will produce $2 \mathrm{x}$ the amount of mols per hour according to this equation:

$\mathrm{H}_{2} \mathrm{SO}_{4}+2 \mathrm{NaOH} \rightarrow 2 \mathrm{H}_{2} \mathrm{O}+\mathrm{Na}_{2} \mathrm{SO}_{4}$

Mols of water produced $=12,008.3 \mathrm{~mol} \mathrm{H}_{2} \mathrm{SO}_{4} / \mathrm{hr} \cdot 2=24,017 \mathrm{~mol}$ water $/ \mathrm{hr}$

$24,017 \mathrm{~mol} \mathrm{water} / \mathrm{hr} \cdot 0.018 \mathrm{~kg}$ water $/ \mathrm{mol}=432 \mathrm{~kg}$ water produced $/ \mathrm{hr}$

Input \#4 - sulfuric acid

Initial pOH:

$958 \mathrm{~kg} \mathrm{NaOH} / \mathrm{hr}$ in $4.54 \times 10^{\wedge} 6 \mathrm{~L} / \mathrm{hr}$

$958 \mathrm{~kg} \mathrm{NaOH} / 0.040 \mathrm{~kg} / \mathrm{mol}=23,950 \mathrm{~mol} / \mathrm{hr}$

$23,950 \mathrm{~mol} / \mathrm{hr} / 4.54 \times 10^{\wedge} 6 \mathrm{~L} / \mathrm{hr}=0.0053 \mathrm{~mol} \mathrm{NaOH} / \mathrm{L}$

$\mathrm{NaOH} \rightarrow \mathrm{Na}^{+}+\mathrm{OH}^{-}\left(1 \mathrm{~mol}\right.$ of $\mathrm{NaOH}$ to $\mathrm{mol}$ of $\left.\mathrm{OH}^{-}\right)$

$\mathrm{pOH}=-\log 10\left[\mathrm{OH}^{-}\right]$

$\mathrm{pOH}=-\log 10[0.0053 \mathrm{~mol} / \mathrm{L}]$

$\mathrm{pOH}=2.28$

To get to $\mathrm{pH}$ of 5 :

$0.0053 \mathrm{~mol} / \mathrm{L} \rightarrow 0.00001 \mathrm{~mol} / \mathrm{L}$ requires reduction of $0.00529 \mathrm{~mol}$ hydroxyl ions $/ \mathrm{L}$ 
$0.00529 \mathrm{~mol}$ hydroxyl ions $/ \mathrm{L} \cdot 4.54 \times 10^{\wedge} 6 \mathrm{~L} \mathrm{NaOH}$ solution $/ \mathrm{hr}=24,017 \mathrm{~mol}$ hydroxyl ions to be $\mathrm{removed} / \mathrm{hr}$

Need the same mols of $\mathrm{NaOH}$ as hydroxide ions according to this equation:

$\mathrm{NaOH} \rightarrow \mathrm{Na}^{+}+\mathrm{OH}^{-}\left(1 \mathrm{~mol}\right.$ of $\mathrm{NaOH}$ to $\mathrm{mol}$ of $\left.\mathrm{OH}^{-}\right)$

Need $1 / 2$ the mols of $\mathrm{H}_{2} \mathrm{SO}_{4}$ than of $\mathrm{NaOH}$ according to this equation:

$\mathrm{H}_{2} \mathrm{SO}_{4}+2 \mathrm{NaOH} \rightarrow 2 \mathrm{H}_{2} \mathrm{O}+\mathrm{Na}_{2} \mathrm{SO}_{4}$

Mols of $\mathrm{H}_{2} \mathrm{SO}_{4}$ required: $0.5 \cdot 24,017 \mathrm{~mol}$ hydroxyl ions/hr

$12,008.3 \mathrm{~mol} \mathrm{H}_{2} \mathrm{SO}_{4} / \mathrm{hr} \cdot 0.098 \mathrm{~kg} \mathrm{H}_{2} \mathrm{SO}_{4} / \mathrm{mol}=1,176.8 \mathrm{~kg} \mathrm{H}_{2} \mathrm{SO}_{4} / \mathrm{hr}$

A.2.2 CEN 01 - Centrifuge to remove wastewater (input \#8, output \#3)

Details/Assumptions:

- $\quad 1000 \mathrm{~kg}$ of water $/ \mathrm{m}^{3}$ (based on $1 \mathrm{~kg} / \mathrm{L}$ water density)

- mass of algae is insignificant compared to the weight of water

- lose $5 \%$ of water from flocculation

- EVODOS centrifuge: $1.2 \mathrm{kWh} / \mathrm{m}^{3}$ water (power required for centrifuge) (EVODOS, 2011)

- Increase solid content to 30\% (EVODOS, 2011)

Calculations:

Input \#8 - energy for centrifuge

$1.09 \times 10^{\wedge} 8 \mathrm{~L}$ water/day through the centrifuge

$1.2 \mathrm{kWh} / \mathrm{m}^{3} \cdot 1.09 \times 10^{\wedge} 5 \mathrm{~m}^{3} /$ day $=130,800 \mathrm{kWh}$ per day

$5,450 \mathrm{kWh}$ (over an hour) 


\section{Output \#3 - wastewater}

$1.09 \times 10^{\wedge} 8 \mathrm{~L}$ wastewater/day $\cdot 0.7=7.63 \times 10^{\wedge} 7 \mathrm{~L}$ wastewater/day

A.2.3 DRY 01 - Dryer to dry all microalgae (input \#11)

Details/Assumptions:

- $\quad 3.2 \mathrm{kWh} / \mathrm{kg}$ dried algae based on a $30 \%$ solid content beforehand (Petrick et al., 2013)

Calculations:

$3.2 \mathrm{kWh} / \mathrm{kg}$ dried algae $\cdot 10,000,000 \mathrm{~kg}$ dried algae/year $=3.2 \times 10^{\wedge} 7 \mathrm{kWh}$ over a year period 4,053 kWh (over an hour - given 47 weeks/year) 
Table A.4: Extraction input/output table

\begin{tabular}{|c|c|c|c|c|c|c|}
\hline Stage & & Inputs & Input amount & & Outputs & Output amount \\
\hline $\begin{array}{l}\text { Air Swept } \\
\text { Pulveriser } \\
\text { (PUV 01) }\end{array}$ & $\begin{array}{l}1 \\
2\end{array}$ & $\begin{array}{l}\text { Algae } \\
\text { Energy }\end{array}$ & $\begin{array}{l}10,000,000 \mathrm{~kg} / \text { year } \\
241.2 \mathrm{MJ} \text { (1 hour) }\end{array}$ & 1 & Algae & $10,000,000 \mathrm{~kg} /$ year \\
\hline $\begin{array}{l}\text { Solvent } \\
\text { addition } \\
\text { (CSTR 01) }\end{array}$ & $\begin{array}{l}3 \\
4 \\
5 \\
6 \\
7\end{array}$ & $\begin{array}{l}\text { Algae } \\
\text { Energy } \\
\text { Heat } \\
\text { Hexane } \\
\text { Ethanol }\end{array}$ & $\begin{array}{l}\text { 10,000,000 kg/year } \\
\mathbf{1 8 4 . 8 4 ~ k W h ~ ( 1 ~ h o u r ) ~} \\
3,147.15 \mathrm{MJ} \text { (1 hour) } \\
\mathbf{8 , 7 7 6 . 1} \mathbf{~ k g} / \mathbf{h r} \\
975,7 \mathrm{~kg} / \mathrm{hr}\end{array}$ & $\begin{array}{l}2 \\
3\end{array}$ & $\begin{array}{l}\text { Lipids } \\
\text { Ligno }+ \text { protein }\end{array}$ & $\begin{array}{l}3,500,000 \mathrm{~kg} / \text { year } \\
6,500,000 \mathrm{~kg} / \text { year }\end{array}$ \\
\hline $\begin{array}{l}\text { Centrifuge } \\
\text { (CEN 02) }\end{array}$ & $\begin{array}{l}8 \\
9 \\
10 \\
11 \\
12\end{array}$ & $\begin{array}{l}\text { Lipids } \\
\text { Ligno + protein } \\
\text { Hexane } \\
\text { Ethanol } \\
\text { Energy }\end{array}$ & $\begin{array}{l}3,500,000 \mathrm{~kg} / \text { year } \\
\mathbf{6 , 5 0 0 , 0 0 0 ~ k g / y e a r} \\
8,776.1 \mathrm{~kg} / \mathrm{hr} \\
\mathbf{9 7 5 , 7} \mathbf{~ k g} / \mathbf{h r} \\
20.1 \mathrm{kWh} \text { (for } 1 \text { hour) }\end{array}$ & $\begin{array}{l}4 \\
5\end{array}$ & $\begin{array}{l}\text { Lipids } \\
\text { Ligno + Protein }\end{array}$ & $\begin{array}{l}3,500,000 \mathrm{~kg} / \text { year } \\
6,500,000 \mathrm{~kg} / \text { year }\end{array}$ \\
\hline $\begin{array}{l}\text { Evaporator } \\
(\text { EVP 01) }\end{array}$ & $\begin{array}{l}13 \\
14 \\
15\end{array}$ & $\begin{array}{l}\text { Lipids } \\
\text { Hexane } \\
\text { Heat (Q) }\end{array}$ & $\begin{array}{l}3,500,000 \mathrm{~kg} / \text { year } \\
\mathbf{8 , 7 7 6 . 1} \mathbf{~ k g} / \mathbf{h r} \\
2,949 \mathrm{MJ} / \mathrm{hr}\end{array}$ & $\begin{array}{l}6 \\
7\end{array}$ & $\begin{array}{l}\text { Lipids } \\
\text { Hexane }\end{array}$ & $\begin{array}{l}3,500,000 \mathrm{~kg} / \text { year } \\
8,776.1 \mathrm{~kg} / \mathrm{hr}\end{array}$ \\
\hline $\begin{array}{l}\text { Condenser } \\
\text { (CND 01) }\end{array}$ & $\begin{array}{l}16 \\
17\end{array}$ & $\begin{array}{l}\text { Hexane } \\
\text { Cooling (Q) }\end{array}$ & $\begin{array}{l}8,776.1 \mathrm{~kg} / \mathrm{hr} \\
2,949 \mathrm{MJ} / \mathrm{hr}\end{array}$ & 8 & Hexane & $8,776.1 \mathrm{~kg} / \mathrm{hr}$ \\
\hline $\begin{array}{l}\text { Evaporator } \\
\text { (EVP 07) }\end{array}$ & $\begin{array}{l}18 \\
19 \\
\end{array}$ & $\begin{array}{l}\text { Ethanol } \\
\text { Heat }(Q)\end{array}$ & $\begin{array}{l}975,7 \mathrm{~kg} / \mathrm{hr} \\
818 \mathrm{MJ} / \mathrm{hr}\end{array}$ & 9 & Ethanol & $975,7 \mathrm{~kg} / \mathrm{hr}$ \\
\hline $\begin{array}{l}\text { Condenser } \\
\text { (CND 07) }\end{array}$ & $\begin{array}{l}20 \\
21\end{array}$ & $\begin{array}{l}\text { Ethanol } \\
\text { Cooling (Q) }\end{array}$ & $\begin{array}{l}975,7 \mathrm{~kg} / \mathrm{hr} \\
818 \mathrm{MJ} / \mathrm{hr}\end{array}$ & 10 & Ethanol & $975,7 \mathrm{~kg} / \mathrm{hr}$ \\
\hline
\end{tabular}

\section{A.3.1 PUV 01 - Air swept pulveriser (input \#2)}

Details/Assumptions: 
- $\quad$ Microalgal cell size: 5.3 micron $(\mathrm{Hu}, 2014)$

- $\quad$ Air swept pulverisers can produce smaller particle sizes (Towers, 2016)

- Jacobson pulverizer can produce particles below 37 micron (Carter Day International Inc., 2012)

- Jacobson pulverizer operates at 50-125 HP (Carter Day International Inc., 2012)

- Capacity for a similar product (to the Jacobson pulverizer) $1200 \mathrm{~kg} / \mathrm{hr}$ with similar power requirement (75 - $100 \mathrm{HP}$ ) (Premium Pulman PVT Ltd., 2017)

- $\quad$ Using $90 \mathrm{HP}=6 \mathbf{6 7}$

Calculations:

$67 \mathrm{~kJ} / \mathrm{s} \cdot 3600 \mathrm{~s}=241,200 \mathrm{~kJ}=241.2 \mathrm{MJ}$ (each hour)

A.3.2 CSTR 01 - Continuous Stirred Tank Reactor for separation mixing (input \#4, 5, 6, 7, output \#2, 3)

Details/Assumptions:

- Several studies have used hexane for extraction of oil in a similar process chains (Lardon, Hélias, Sialve, Steyer, \& Bernard, 2009; Nanaki \& Koroneos, 2012; Stephenson et al., 2010)

- Hexane molecular weight: $86.18 \mathrm{~g} / \mathrm{mol}$

- Hexane boiling point: $68^{\circ} \mathrm{C}($ Green \& Perry 2007)

- Dry extraction power requirement: $0.417 \mathrm{kWh} / \mathrm{kg}$ extracted oil; heat requirement: $7.1 \mathrm{MJ} / \mathrm{kg}$ extracted oil; hexane loss: 0.015 $\mathrm{kg} / \mathrm{kg}$ extracted oil calculated from Table 3 of (Lardon et al., 2009)

- Hexane/ethanol solvent mixture with hexane:ethanol at 9:1 by weight with solvent to oil ratio of 22:1 by weight with a $90 \%$ recovery of oil (Batan et al. 2010)

- Assume hexane and ethanol loss in the same ratio (9:1) 
- $35 \mathrm{wt} \%$ lipids (realistic lipid percentages based on values found in Table 2.4 of this document and optimal nutrients)

Calculations:

Input \#4, 5, $6 \& 7$

Power and heat requirements:

3,500,000 kg lipids/year $\cdot 1$ year $/ 47$ weeks $\cdot 1$ week $/ 7$ days $\cdot 1$ day $/ 24$ hours $=443.26 \mathrm{~kg}$ lipids $/ \mathrm{hr}$ enter the bioreactor

$0.417 \mathrm{kWh} / \mathrm{kg} \cdot 443.26 \mathrm{~kg} / \mathrm{hr}=184.84 \mathrm{kWh}(665.42 \mathrm{MJ})$

$7.1 \mathrm{MJ} / \mathrm{kg} \cdot 443.26 \mathrm{~kg} / \mathrm{hr}=3,147.15 \mathrm{MJ}$

Hexane and ethanol loss:

$0.015 \mathrm{~kg}$ hexane loss $/ \mathrm{kg}$ extracted oil $\cdot 443.26 \mathrm{~kg}$ extracted oil $/ \mathrm{hr}=6.65 \mathrm{~kg}$ hexane $/ \mathrm{hr}$ (close to $2 \mathrm{~g}$ hexane loss $/ \mathrm{kg}$ dry algae in Lardon et al. 2009)

$1,266.5 \mathrm{~kg}$ algae $/ \mathrm{hr} \cdot 2 \mathrm{~g}$ hexane loss $/ \mathrm{kg}$ dry algae $=2,533 \mathrm{~g}$ hexane loss $/ \mathrm{hr}=2.5 \mathrm{~kg}$ of hexane loss $/ \mathrm{hr}$ (using Lardon et al. 2009)

Hexane $(x)+$ ethanol $(y)=6.65 \mathrm{~kg}$

$9 \mathrm{y}=\mathrm{x}(9: 1$ hexane/ethanol ratio $)$

Hexane loss $=5.99 \mathrm{~kg} / \mathrm{hr}$

Ethanol loss $=0.65 \mathrm{~kg} / \mathrm{hr}$ 
Amount of total hexane required for circulation purposes:

$443.26 \mathrm{~kg}$ oil $/ \mathrm{hr} \cdot 22=9,751.72 \mathrm{~kg}$ solvent required $(22: 1$ solvent/oil ratio $)$

Hexane $(\mathrm{x})+$ ethanol $(\mathrm{y})=9,751.72$

$9 \mathrm{y}=\mathrm{x}(9: 1$ hexane/ethanol ratio $)$

Ethanol required $=975.12 \mathrm{~kg} / \mathrm{hr}$

Hexane required $=8,776.1 \mathrm{~kg} / \mathrm{hr}$

\section{Output \#2 \& 3}

Calculations:

$10,000,000 \mathrm{~kg}$ mircroalgae/year $\cdot 0.35$ lipid content $=3,500,000 \mathrm{~kg}$ lipids $/ \mathrm{kg}$ of microalgae

$65 \%$ lignocellulosic biomass and protein left over $=6,500,000 \mathrm{~kg}$ lingo+protein $/ \mathrm{kg}$ of microalgae

A.3.3 CEN 02 - Centrifuge to separate lipids from rest of microalgal biomass (input \#12)

Details/Assumptions:

- Average density of Chlorella Vulgaris is $0.57 \mathrm{~g} / \mathrm{cm}^{3}(\mathrm{Hu}, 2014), 0.57 \mathrm{~g} / \mathrm{cm}^{3}=570 \mathrm{~kg} / \mathrm{m}^{3}$

- Density of hexane: $659 \mathrm{~kg} / \mathrm{m}^{3}$ (Green \& Perry 2007)

- Density of ethanol: $0.79 \mathrm{~kg} / \mathrm{L}$ at $15^{\circ} \mathrm{C}$ (Table 2.2 in section 2.1 .2 ) 


\section{Calculations:}

$10,000,000 \mathrm{~kg}$ microalgae/year/47 week/year/7days a week $/ 24$ hours $/$ day $=1.27 \times 10^{\wedge} 3 \mathrm{~kg}$ of microalgae $/ \mathrm{hr}$ $1.27 \times 10^{\wedge} 3 \mathrm{~kg}$ of microalgae $/ \mathrm{hr} / 570 \mathrm{~kg} / \mathrm{m}^{3}=2.23 \mathrm{~m}^{3}$

$8,776.1 \mathrm{~kg}$ hexane $/ \mathrm{hr} / 659 \mathrm{~kg} / \mathrm{m}^{3}=13.3 \mathrm{~m}^{3}$

$975.12 \mathrm{~kg}$ ethanol $/ \mathrm{hr} / 0.79 \mathrm{~kg} / \mathrm{L} \cdot 1 \mathrm{~L} / 0.001 \mathrm{~m}^{3}=1.2 \mathrm{~m}^{3}$

Total volume $\left(16.7 \mathrm{~m}^{3}\right)$ to run through the centrifuge in an hour $1.2 \mathrm{kWh} / \mathrm{m} 3(\mathrm{EVODOS}, 2011) \cdot 16.7 \mathrm{~m}^{3}=20.1 \mathrm{kWh}$ (for 1 hour)

\section{A.3.4 EVP 01 - Evaporate hexane from lipid stream (input \#15)}

Details/Assumptions:

- Using a falling film evaporator as per Figure A.1

- Circulation energy included in the Pump Energy section (Appendix B)

- Using NG for heat source 


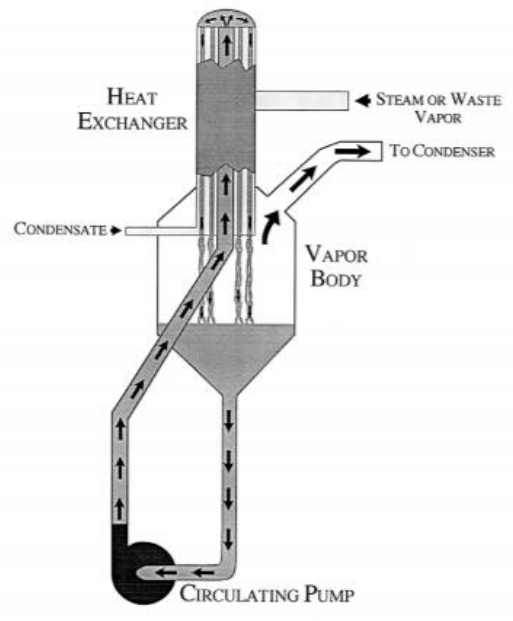

Figure A.1: Falling film evaporator (The Dupps Company, 2017)

Calculations:

$\mathrm{H} 1=\int(341 \mathrm{~K}-298 \mathrm{~K}) \mathrm{Cp}(\mathrm{l})(\mathbf{T G}) \mathrm{dT}$

$\mathrm{H} 1=\int(341 \mathrm{~K}-298 \mathrm{~K})\left(\mathrm{Ai}+\mathrm{BiT}+\mathrm{CiT}^{\wedge} 2\right) \mathrm{dT}($ in $\mathrm{J} / \mathrm{kmol} \cdot \mathrm{K})$

$\mathrm{H} 1=1475.1 \mathrm{~T}-\left(0.71 \mathrm{~T}^{\wedge} 2\right) / 2+\left(5.85 \mathrm{~T}^{\wedge} 3\right) / 3 * 10^{\wedge} 3$

$\mathrm{H} 1=1475.1(341-298)-\left(0.71\left(341^{\wedge} 2-298^{\wedge} 2\right)\right) / 2+\left(5.85\left(341^{\wedge} 3-298^{\wedge} 3\right)\right) / 3 \cdot 10^{\wedge} 3$

$\mathrm{H} 1=61,987 \mathrm{~J} / \mathrm{kmol}$

$\mathrm{H} 1=0.068 \mathrm{~kJ} / \mathrm{mol}$

$\mathrm{H} 2=\int(341 \mathrm{~K}-298 \mathrm{~K}) \mathrm{Cp}(\mathrm{l})(\mathbf{H E X}) \mathrm{dT}+\Delta \mathbf{H}$ vap

Assume $\Delta \mathrm{H}$ vap supersedes 
$\mathrm{H} 2=29.24 \mathrm{~kJ} / \mathrm{mol}$

Mol of hexane:

$8,776.1 \mathrm{~kg} / 0.086 \mathrm{~kg} / \mathrm{mol}=102,048 \mathrm{~mol}$ of hexane $/ \mathrm{hr}$

$\mathrm{Q}=\Sigma$ Internal enthalpy rate $=\mathrm{n} 1 \mathrm{H} 1+\mathrm{n} 2 \mathrm{H} 2$

$\mathrm{Q}=(501 \mathrm{~mol} / \mathrm{hr})(0.062 \mathrm{~kJ} / \mathrm{mol})+(102,048 \mathrm{~mol} / \mathrm{hr})(28.9 \mathrm{~kJ} / \mathrm{mol})$

$\mathrm{Q}=31 \mathrm{~kJ} / \mathrm{hr}+2,949,178 \mathrm{~kJ} / \mathrm{hr}$

$\mathrm{Q}=2,949 \mathrm{MJ} / \mathrm{hr}$

Table A.5: EVP01 - hexane evaporation from lipid stream

\begin{tabular}{|l|l|l|l|l|}
\hline Product & Mass flow (kg/hr) & Molar flow (kmol/hr) (ni) & $\begin{array}{l}\text { Internal Enthalpy } \\
\text { designate (J/kmol) }\end{array}$ & $\begin{array}{l}\text { Internal Enthalpy value } \\
\text { (based on scenario) }\end{array}$ \\
\hline $\begin{array}{l}\text { Triglyceride } \\
\text { (lipid for inlet) }\end{array}$ & 443.26 & 0.501 & $\mathrm{H} 1$ & $0.068 \mathrm{~kJ} / \mathrm{mol}$ \\
\hline $\begin{array}{l}\text { Hexane }(86.18 \\
\mathrm{g} / \mathrm{mol})\end{array}$ & $8,776.1 \mathrm{~kg} / \mathrm{hr}$ & $102,048 \mathrm{~mol} / \mathrm{hr}$ & $\mathrm{H} 2$ & $28.9 \mathrm{~kJ} / \mathrm{mol}$ \\
\hline
\end{tabular}

A.3.5 CND 01 - Condenser for hexane (input \#15)

$\mathrm{H} 2=-\int(341 \mathrm{~K}-339 \mathrm{~K}) \mathrm{Cp}(\mathrm{l})(\mathbf{H E X}) \mathrm{dT}-\mathbf{\Delta} \mathbf{H}$ vap

Assume $\Delta \mathrm{H}$ vap supersedes

$\mathrm{H} 2=-28.9 \mathrm{~kJ} / \mathrm{mol}$ 
$\mathrm{Q}=\Sigma$ Internal enthalpy rate $=\mathrm{n} 2 \mathrm{H} 2$

$\mathrm{Q}=102,048 \mathrm{~mol} / \mathrm{hr} \cdot(-28.9 \mathrm{~kJ} / \mathrm{mol})$

$\mathrm{Q}=-2,949 \mathrm{MJ} / \mathrm{hr}$

\section{A.3.6 EVP 07 - Evaporator for ethanol (input \#19)}

Details/Assumptions:

- $\quad$ Using a falling film evaporator as per Figure A.1

- Circulation energy included in the Pump Energy section (Appendix B)

- $\quad \mathrm{T}_{\mathrm{in}}=68^{\circ} \mathrm{C}$ (from the evaporation of hexane), $\mathrm{T}_{\text {out }}=79^{\circ} \mathrm{C}$ (boiling point of ethanol)

- Using NG for heat source

$\mathrm{H}=\int(352 \mathrm{~K}-341 \mathrm{~K}) \mathrm{Cp}(\mathrm{l})(\mathbf{E T H}) \mathrm{dT}+\Delta \mathrm{H}$ vaporization (evaporating bio-ethanol)

Assume $\Delta \mathrm{H}$ vap supersedes

$\mathrm{H}=38.6 \mathrm{~kJ} / \mathrm{gmol}$

Mol of ethanol:

$975.12 \mathrm{~kg} / \mathrm{hr} / 0.046 \mathrm{~kg} / \mathrm{mol}=21,198 \mathrm{~mol}$ ethanol $/ \mathrm{hr}$

$\mathrm{Q}=\Sigma$ Internal enthalpy rate $=\mathrm{nH}$ 
$\mathrm{Q}=21,198 \mathrm{~mol} / \mathrm{hr} \cdot 38.6 \mathrm{~kJ} / \mathrm{gmol}$

$\mathrm{Q}=818,243 \mathrm{~kJ} / \mathrm{hr}$

$\mathrm{Q}=818 \mathrm{MJ} / \mathrm{hr}$

Table A.6: EVP07 - ethanol evaporation from lipid stream

\begin{tabular}{|l|l|l|l|l|}
\hline Product & Mass flow (kg/hr) & Molar flow (kmol/hr) (ni) & $\begin{array}{l}\text { Internal Enthalpy } \\
\text { designate (J/kmol) }\end{array}$ & $\begin{array}{l}\text { Internal Enthalpy value } \\
\text { (based on scenario) }\end{array}$ \\
\hline $\begin{array}{l}\text { Triglyceride } \\
\text { (lipid for inlet) }\end{array}$ & 443.26 & 0.501 & $\mathrm{H} 1$ & $0.068 \mathrm{~kJ} / \mathrm{mol}$ \\
\hline $\begin{array}{l}\text { Ethanol }(46 \\
\text { g/mol) }\end{array}$ & $975.12 \mathrm{~kg} / \mathrm{hr}$ & $21,198 \mathrm{~mol} / \mathrm{hr}$ & $\mathrm{H} 2$ & $38.6 \mathrm{~kJ} / \mathrm{gmol}$ \\
\hline
\end{tabular}

\section{A.3.7 CND 07 - Condenser for ethanol (input \#21)}

Assume $\Delta \mathrm{H}$ vap supersedes

$\mathrm{H}=-38.6 \mathrm{~kJ} / \mathrm{gmol}$

$\mathrm{Q}=\Sigma$ Internal enthalpy rate $=\mathrm{nH}$

$\mathrm{Q}=21,198 \mathrm{~mol} / \mathrm{hr} *-38.6 \mathrm{~kJ} / \mathrm{gmol}$

$\mathrm{Q}=-818,243 \mathrm{~kJ} / \mathrm{hr}$

$\mathrm{Q}=-818 \mathrm{MJ} / \mathrm{hr}$ 
Table A.7: BD process input/output table

\begin{tabular}{|c|c|c|c|c|c|c|}
\hline Stage & & Inputs & Input amount & & Outputs & Output amount \\
\hline $\begin{array}{l}\text { Positive } \\
\text { displacement } \\
\text { heat pump } \\
\text { (CMP 01) }\end{array}$ & $\begin{array}{l}1 \\
2 \\
3\end{array}$ & $\begin{array}{l}\text { Power/Energy } \\
\text { Triglycerides (Lipids) } \\
\text { Methanol }\end{array}$ & $\begin{array}{l}3.6 \mathrm{~kW} / 3.6 \mathrm{kWh} \\
\mathbf{4 4 3 . 2 6 ~ k g / h r} \\
144.25 \mathrm{~kg} / \mathrm{hr}\end{array}$ & $\begin{array}{l}1 \\
2\end{array}$ & $\begin{array}{l}\text { Triglycerides } \\
\text { (Lipids) } \\
\text { Methanol }\end{array}$ & $\begin{array}{l}\mathbf{4 4 3 . 2 6} \mathbf{~ k g} / \mathrm{hr} \\
144.25 \mathrm{~kg} / \mathrm{hr}\end{array}$ \\
\hline $\begin{array}{l}\text { Transesterific } \\
\text { ation (CSTR } \\
\text { 02) }\end{array}$ & $\begin{array}{l}4 \\
5 \\
6\end{array}$ & $\begin{array}{l}\text { Triglycerides (Lipids) } \\
\text { Methanol } \\
\text { Heat energy (Q) }\end{array}$ & $\begin{array}{l}443.26 \mathrm{~kg} / \mathrm{hr} \\
\mathbf{1 4 4 . 2 5 ~ k g / h r} \\
42.3 \mathrm{~kW}\end{array}$ & $\begin{array}{l}3 \\
4 \\
5 \\
6 \\
7 \\
8\end{array}$ & $\begin{array}{l}\text { Triglycerides } \\
\text { Methanol } \\
\text { Diglycerides } \\
\text { Monoglycerides } \\
\text { Methylesters } \\
\text { Glycerol }\end{array}$ & $\begin{array}{l}0.11 \mathrm{~kg} / \mathrm{hr} \\
97.70 \mathrm{~kg} / \mathrm{hr} \\
1.59 \mathrm{~kg} / \mathrm{hr} \\
5.17 \mathrm{~kg} / \mathrm{hr} \\
429.53 \mathrm{~kg} / \mathrm{hr} \\
\mathbf{5 3 . 3 4} \mathrm{kg} / \mathrm{hr}\end{array}$ \\
\hline $\begin{array}{l}\text { Cooler (CLR } \\
01)\end{array}$ & $\begin{array}{l}7 \\
8 \\
9 \\
10 \\
11 \\
12 \\
13\end{array}$ & $\begin{array}{l}\text { Triglycerides } \\
\text { Methanol } \\
\text { Diglycerides } \\
\text { Monoglycerides } \\
\text { Methylesters } \\
\text { Glycerol } \\
\text { Cooling energy }\end{array}$ & $\begin{array}{l}0.11 \mathrm{~kg} / \mathrm{hr} \\
\mathbf{9 7 . 7 0} \mathbf{~ k g} / \mathrm{hr} \\
1.59 \mathrm{~kg} / \mathrm{hr} \\
\mathbf{5 . 1 7} \mathbf{~ k g} / \mathbf{h r} \\
429.53 \mathrm{~kg} / \mathrm{hr} \\
\mathbf{5 3 . 3 4} \mathbf{~ k g} / \mathbf{h r} \\
50 \mathrm{kWh}\end{array}$ & $\begin{array}{l}10 \\
11 \\
12 \\
13 \\
14 \\
15\end{array}$ & $\begin{array}{l}\text { Triglycerides } \\
\text { Methanol } \\
\text { Diglycerides } \\
\text { Monoglycerides } \\
\text { Methylesters } \\
\text { Glycerol }\end{array}$ & $\begin{array}{l}0.11 \mathrm{~kg} / \mathrm{hr} \\
\mathbf{9 7 . 7 0} \mathrm{kg} / \mathrm{hr} \\
1.59 \mathrm{~kg} / \mathrm{hr} \\
\mathbf{5 . 1 7} \mathrm{kg} / \mathrm{hr} \\
429.53 \mathrm{~kg} / \mathrm{hr} \\
\mathbf{5 3 . 3 4} \mathrm{kg} / \mathrm{hr}\end{array}$ \\
\hline $\begin{array}{l}\text { Evaporator } \\
\text { (EVP 02) }\end{array}$ & $\begin{array}{l}14 \\
15 \\
16 \\
17 \\
18 \\
19\end{array}$ & $\begin{array}{l}\text { Triglycerides } \\
\text { Methanol } \\
\text { Diglycerides } \\
\text { Monoglycerides } \\
\text { Methylesters } \\
\text { Heat energy }\end{array}$ & $\begin{array}{l}0.11 \mathrm{~kg} / \mathrm{hr} \\
97.70 \mathrm{~kg} / \mathrm{hr} \\
1.59 \mathrm{~kg} / \mathrm{hr} \\
5.17 \mathrm{~kg} / \mathrm{hr} \\
429.53 \mathrm{~kg} / \mathrm{hr} \\
0\end{array}$ & 16 & Methanol & $75.88 \mathrm{~kg} / \mathrm{hr}$ \\
\hline $\begin{array}{l}\text { Condenser } \\
\text { (CDN 02) }\end{array}$ & $\begin{array}{l}20 \\
21\end{array}$ & $\begin{array}{l}\text { Methanol } \\
\text { Cooling energy }\end{array}$ & $\begin{array}{l}75.88 \mathrm{~kg} / \mathrm{hr} \text { (from } \\
\text { EVP 02) }\end{array}$ & 17 & Methanol & $\begin{array}{l}94.39 \mathrm{~kg} / \mathrm{hr} \\
\text { (recovered) }\end{array}$ \\
\hline
\end{tabular}




\begin{tabular}{|c|c|c|c|c|c|c|}
\hline & 22 & Methanol sourced & 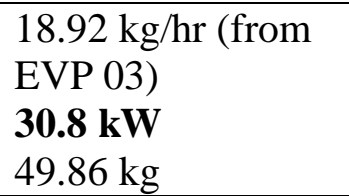 & & & \\
\hline $\begin{array}{l}\text { Centrifuge } \\
\text { (CEN 03) }\end{array}$ & $\begin{array}{l}23 \\
24 \\
25 \\
26 \\
27 \\
28 \\
29\end{array}$ & $\begin{array}{l}\text { Triglycerides } \\
\text { Methanol } \\
\text { Diglycerides } \\
\text { Monoglycerides } \\
\text { Methylesters } \\
\text { Glycerol } \\
\text { Energy }\end{array}$ & $\begin{array}{l}0.11 \mathrm{~kg} / \mathrm{hr} \\
\mathbf{2 1 . 8 2} \mathbf{~ k g} / \mathbf{h r} \\
1.59 \mathrm{~kg} / \mathrm{hr} \\
\mathbf{5 . 1 7} \mathbf{~ k g} / \mathbf{h r} \\
429.53 \mathrm{~kg} / \mathrm{hr} \\
\mathbf{5 3 . 3 4} \mathbf{~ k g} / \mathbf{h r} \\
0.746 \mathrm{kWh}(1 \text { hour})\end{array}$ & $\begin{array}{l}18 \\
19 \\
20 \\
21 \\
22\end{array}$ & $\begin{array}{l}\text { Triglycerides } \\
\text { Methanol } \\
\text { Diglycerides } \\
\text { Monoglycerides } \\
\text { Methylesters }\end{array}$ & $\begin{array}{l}0.11 \mathrm{~kg} / \mathrm{hr} \\
2.9 \mathrm{~kg} / \mathrm{hr} \\
1.59 \mathrm{~kg} / \mathrm{hr} \\
5.17 \mathrm{~kg} / \mathrm{hr} \\
429.53 \mathrm{~kg} / \mathrm{hr}\end{array}$ \\
\hline $\begin{array}{l}\text { Evaporator } \\
\text { (EVP 03) }\end{array}$ & $\begin{array}{l}30 \\
31 \\
32\end{array}$ & $\begin{array}{l}\text { Glycerol } \\
\text { Methanol } \\
\text { Heat }(Q)\end{array}$ & $\begin{array}{l}53.34 \mathrm{~kg} / \mathrm{hr} \\
\mathbf{2 1 . 8 2} \mathbf{~ k g} / \mathbf{h r} \\
22 \mathrm{MJ} / \mathrm{hr}\end{array}$ & $\begin{array}{l}23 \\
24\end{array}$ & $\begin{array}{l}\text { Glycerol } \\
\text { Methanol }\end{array}$ & $\begin{array}{l}53.34 \mathrm{~kg} / \mathrm{hr} \\
18.92 \mathrm{~kg} / \mathrm{hr}\end{array}$ \\
\hline $\begin{array}{l}\text { Evaporator } \\
\text { (EVP 04) }\end{array}$ & $\begin{array}{l}33 \\
34 \\
35 \\
36 \\
37 \\
38\end{array}$ & $\begin{array}{l}\text { Triglycerides } \\
\text { Diglycerides } \\
\text { Monoglycerides } \\
\text { Methylesters } \\
\text { Methanol } \\
\text { Heat energy (Q) }\end{array}$ & 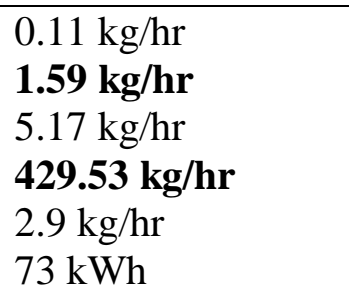 & $\begin{array}{l}25 \\
26 \\
27 \\
28 \\
29\end{array}$ & $\begin{array}{l}\text { Triglycerides } \\
\text { Diglycerides } \\
\text { Monoglycerides } \\
\text { Methylesters } \\
\text { Methanol }\end{array}$ & $\begin{array}{l}0.11 \mathrm{~kg} / \mathrm{hr} \\
\mathbf{1 . 5 9} \mathrm{kg} / \mathrm{hr} \\
5.17 \mathrm{~kg} / \mathrm{hr} \\
\mathbf{4 2 9 . 5 3 ~ k g / h r} \\
2.9 \mathrm{~kg} / \mathrm{hr}\end{array}$ \\
\hline $\begin{array}{l}\text { Condenser } \\
\text { (CND 03) }\end{array}$ & $\begin{array}{l}39 \\
40\end{array}$ & $\begin{array}{l}\text { Methylesters } \\
\text { Cooling energy (Q) }\end{array}$ & $\begin{array}{l}429.53 \mathrm{~kg} / \mathrm{hr} \\
35 \mathrm{kWh}\end{array}$ & 30 & Methylesters & $429.53 \mathrm{~kg} / \mathrm{hr}$ \\
\hline
\end{tabular}

\section{A.4.1 CMP 01 - Compressor for TG feed (input \#1, 3)}

Details/Assumptions:

- Supercritical methanol is the process used for transesterification for this study

- Conversion of oil to FAME is rapid (i.e. less than 3 minutes) with a $98 \%$ conversion at 10-20 MPa, between 375 and $400^{\circ} \mathrm{C}$ with a methanol:oil ratio of 3:1 to 6:1 (Bernal et al., 2012; Marulanda et al., 2010; Pinnarat \& Savage, 2008)

- This study assumes reactor pressure increased from $0.1-15 \mathrm{mPa}$ as per above (not dealing with head pressure) 
- Density of TG: $915 \mathrm{~kg} / \mathrm{m}^{3}$ (Green \& Perry, 2007)

- Density of methanol: $810 \mathrm{~kg} / \mathrm{m}^{3}$ (Green \& Perry, 2007)

- $\quad \mathrm{P}_{\text {elect }}(\mathrm{kW})=\mathrm{Q}\left(\mathrm{m}^{3} / \mathrm{hr}\right) \cdot \Delta \mathrm{P}(\mathrm{bar}) / 36 \cdot \mathrm{E}(\% / 100), \mathrm{E}=$ efficiency of (pump, transmission, motor) (Vogelesang, 2008)

- Molar methanol: Molar Triglyceride (9:1) (Liu, 2013)

Calculations:

Input \#1 - power requirement for pressure increase

$\left(443.26 \mathrm{~kg} / \mathrm{hr} / 915 \mathrm{~kg} / \mathrm{m}^{3}\right)+\left(144.25 \mathrm{~kg} / \mathrm{hr} / 810 \mathrm{~kg} / \mathrm{m}^{3}\right)=0.48+0.18 \mathrm{~m}^{3}=0.66 \mathrm{~m}^{3} / \mathrm{hr}$

$\mathrm{P}_{\text {elect }}=0.66 \mathrm{~m}^{3} / \mathrm{hr} \cdot 150 \mathrm{bar} / 36 \cdot 0.75$

$\mathrm{P}_{\text {elect }}=3.6 \mathrm{~kW}$ (power)

If the pump operates for 1 hour then the total energy used is $3.6 \mathrm{kWh}$

Input \#3 - methanol requirement

Molar flow rate of methanol:

Lipids: $443.26 \mathrm{~kg}$ lipids $/$ hour $\cdot 1 \mathrm{kmol} / 885 \mathrm{~kg}=0.5 \mathrm{kmol} / \mathrm{hr}$

Methanol: $9 \cdot 0.5 \mathrm{kmol} / \mathrm{hr}=4.5 \mathrm{kmol} / \mathrm{hr}$

$4.5 \mathrm{kmol} / \mathrm{hr} \cdot 32 \mathrm{~kg} / \mathrm{kmol}=144.25 \mathrm{~kg} / \mathrm{hr}$

A.4.2 CSTR 02 - Transesterification reaction (input \#6, output\# 3-8)

Details/Assumptions:

- Supercritical methanol is the process used for transesterification for this study 
- Molar methanol: Molar Triglyceride (9:1) (Jiuxu Liu, 2013)

- $\quad 443.26 \mathrm{~kg}$ lipids/hr enter the bioreactor (calculated above)

- Dowthern A is used as a heating medium. The heat source for Downthern A is NG in GaBi using LHV of NG

- Loss of refrigeratants (e.g. Downtern A) is assumed to be 5\%

- $\quad$ Molecular weight of TG $=885 \mathrm{~g} / \mathrm{mol}$ (see Table A.8)

- Methanol can be obtained from renewable sources, it is usually derived from natural gas (Knothe, 2010). Therefore, methanol:NG by weight ratio is $1.7: 1$ with density of $\mathrm{NG}$ at $25^{\circ} \mathrm{C}$ and 1 atm at $0.66 \mathrm{~g} / \mathrm{L}$ (Cheng \& Kung, 1994; Haid \& Koss, 2001). NG is used as a substitute for methanol as methanol is not available in GaBi Education.

Calculations:

Output \#3-8 - amount of methanol, glycerides and FAME

Molar flow rate of all inputs:

Lipids: $443.26 \mathrm{~kg}$ lipids/hour $\cdot 1 \mathrm{kmol} / 885 \mathrm{~kg}=\mathbf{0 . 5} \mathbf{~ k m o l} / \mathbf{h r}$

Methanol: $9 \cdot 0.5 \mathrm{kmol} / \mathrm{hr}=\mathbf{4 . 5} \mathbf{~ k m o l} / \mathrm{hr}$

$4.5 \mathrm{kmol} / \mathrm{hr} \cdot 32 \mathrm{~kg} / \mathrm{kmol}=144.25 \mathrm{~kg} / \mathrm{hr}$ (recover enough that only need to supplement 49.86/hr)

Converting methanol to NG for input into GaBi

methanol:NG 1.7:1

$144.25 \mathrm{~kg} / 1.7=84.9 \mathrm{~kg}$ of $\mathrm{NG}$

*Note - steam reforming impacts not included here 
Total inlet mass flow:

$144.25 \mathrm{~kg}$ methanol $/ \mathrm{hr}+443.26 \mathrm{~kg}$ lipid $/ \mathrm{hr}=587.51 \mathrm{~kg} / \mathrm{hr}$

Outlet mass flow calculations based on ratios in Table A.8 (Liu, 2013) (supercritical transesterification process):

Methanol: $587.51 \mathrm{~kg}$ inlet mass $/ \mathrm{hr} \cdot 16.63 \mathrm{wt} \%=97.70 \mathrm{~kg} / \mathrm{hr}$

Glycerol: $587.51 \mathrm{~kg}$ inlet mass $/ \mathrm{hr} \cdot 9.08 \mathrm{wt} \%=53.34 \mathrm{~kg} / \mathrm{hr}$

MG: $587.51 \mathrm{~kg}$ inlet mass $/ \mathrm{hr} \cdot 0.88 \mathrm{wt} \%=5.17 \mathrm{~kg} / \mathrm{hr}$

DG: $587.51 \mathrm{~kg}$ inlet mass $/ \mathrm{hr} \cdot 0.27 \mathrm{wt} \%=1.59 \mathrm{~kg} / \mathrm{hr}$

TG: $587.51 \mathrm{~kg}$ inlet mass $/ \mathrm{hr} \cdot 0.02 \mathrm{wt} \%=0.11 \mathrm{~kg} / \mathrm{hr}$

FAME: $587.51 \mathrm{~kg}$ inlet mass $/ \mathrm{hr} \cdot 73.11 \mathrm{wt} \%=429.53 \mathrm{~kg} / \mathrm{hr}$

\section{$\underline{\text { Input \#6 - Heat }}$}

- Dowthern A - used in liquid phase heat exchanger from $15-400^{\circ} \mathrm{C}$ (can be used as heat exchanger fluid in vapour form from $257^{\circ} \mathrm{C}$ to $\left.400^{\circ} \mathrm{C}\right)$ and freezing point $\left(12^{\circ} \mathrm{C}\right)$ (The DOW Chemical Company, 1997)

- Molecular weight of Dowthern A: $166 \mathrm{~g} / \mathrm{mol}$ (The DOW Chemical Company, 1997)

- Heat capacity of Dowtherm A: $2.7 \mathrm{~kJ} / \mathrm{kg} \cdot \mathrm{K}\left(@ 400^{\circ} \mathrm{C}\right)$ (The DOW Chemical Company, 1997)

- Heat of formation is the energy associated with the formation of the material

- Heat capacity is the ratio of heat added to or removed from a material to the resulting temperature change of the material (J/Kelvin) 
Table A.8: Properties of transesterification products (Anitescu \& Bruno, 2012; Lapurta et al., 2010; Perry et al., 1997)

\begin{tabular}{|l|l|l|l|l|l|}
\hline Product & $\begin{array}{l}\text { Molecular } \\
\text { weight } \\
(\mathbf{g} / \mathbf{m o l})\end{array}$ & $\begin{array}{l}\text { Weight composition of } \\
\text { product ratio } \mathbf{\%} \text { of } \\
\text { total) }(\mathbf{L i u} \mathbf{2 0 1 3})\end{array}$ & $\begin{array}{l}\text { Boiling Point } \\
(* \mathbf{C})\end{array}$ & $\begin{array}{l}\text { Heat of Formation } \\
(\mathbf{k J} / \mathbf{m o l})\end{array}$ & $\begin{array}{l}\text { Heat of } \\
\text { Vaporization } \\
\text { (kJ/mol) }(\mathbf{R e i d} \text { et al. } \\
\mathbf{2 0 0 7})\end{array}$ \\
\hline Glycerol & 92 & 9.08 & 288 & -669.6 & 91.7 \\
\hline Monoglyceride & 356 & 0.879 & 238 & -1147.31 & 151.86 \\
\hline Diglyceride & 621 & 0.2697 & 397.7 & -1651.79 & 222.69 \\
\hline $\begin{array}{l}\text { Triglyceride } \\
\text { (lipid for inlet) }\end{array}$ & 885 & 0.02 & 629 & -2129.07 & 285.77 \\
\hline FAME & 296.5 & 73.1196 & 218.5 & -734.5 & 84.6 \\
\hline Methanol & 32 & 16.6317 & 64.7 & -239.2 & 37.6 \\
\hline Chloroform & 119.38 & & 62 & & 29.24 \\
\hline
\end{tabular}

Table A.9: Mass and molar flows of transesterification process

\begin{tabular}{|c|c|c|c|c|c|c|}
\hline Product & $\begin{array}{l}\text { Input mass } \\
\text { flow }(\mathrm{kg} / \mathrm{hr})\end{array}$ & $\begin{array}{l}\text { Input molar flow } \\
\text { (kmol/hr) (ni) }\end{array}$ & $\begin{array}{l}\text { Output mass } \\
\text { flow }(\mathrm{kg} / \mathrm{hr})\end{array}$ & $\begin{array}{l}\text { Output } \\
\text { molar flow } \\
\text { (kmol/hr) }\end{array}$ & $\begin{array}{l}\text { Internal Enthalpy } \\
\text { designate }(\mathrm{J} / \mathrm{kmol})\end{array}$ & $\begin{array}{l}\text { Internal Enthalpy } \\
\text { value }(\mathrm{J} / \mathrm{kmol})\end{array}$ \\
\hline Glycerol & & & 53.34 & 0.58 & $\mathrm{H} 8$ & 320,028 \\
\hline Monoglyceride & & & 5.17 & 0.01 & $\mathrm{H} 3$ & 534,768 \\
\hline Diglyceride & & & 1.59 & 0.003 & $\mathrm{H} 4$ & 751,121 \\
\hline $\begin{array}{l}\text { Triglyceride } \\
\text { (lipid for inlet) }\end{array}$ & $443.26 \mathrm{~kg} / \mathrm{hr}$ & 0.5 & 0.11 & 0.0001 & $\mathrm{H} 1 / \mathrm{H} 5$ & 966,688 \\
\hline FAME & & & 429.53 & 1.45 & H6 & 326,656 \\
\hline Methanol & $144.25 \mathrm{~kg} / \mathrm{hr}$ & 4.51 & 97.70 & 3.05 & $\mathrm{H} 2 / \mathrm{H} 7$ & 20,827 \\
\hline
\end{tabular}


Table A.10: Heat capacity coefficients for liquids ((Zeng et al., 2014), Cp (liquid component) $=\mathrm{Ai}+\mathrm{BiT}^{\mathrm{T}} \mathrm{CiT} \mathrm{C}^{\wedge}(\mathrm{in} \mathrm{J} / \mathrm{kmol} \cdot \mathrm{K})$

\begin{tabular}{|l|l|l|l|}
\hline Component & A & B & $\mathbf{C x 1 0}^{\wedge} \mathbf{3}$ \\
\hline Triglyceride/Lipids & 1475.0791 & -0.7072 & 5.8483 \\
\hline Diglyceride & 1105.3142 & -1.2734 & 6.1244 \\
\hline Monoglyceride & 735.5490 & -1.8397 & 6.4005 \\
\hline MeOL/FAME & 509.4171 & -0.3055 & 2.0693 \\
\hline Glycerol & 365.7840 & -2.4060 & 6.6766 \\
\hline MeOH & 7.2703 & 0.1328 & -0.0610 \\
\hline
\end{tabular}

Table A.11: Heat capacity coefficients for gases ((Sazhin et al., 2014), Cp (gas component) $=\left(\mathrm{Ai}+\mathrm{BiT}+\mathrm{CiT}^{\wedge} 2\right) \cdot 10^{\wedge} 3(\mathrm{in} \mathrm{J} / \mathrm{mol} \cdot \mathrm{K})$

\begin{tabular}{|l|l|l|l|}
\hline Component & A & B & C \\
\hline MeOH/FAME & 1.915 & -0.002163 & 0.00000829 \\
\hline $\mathrm{MeOH}$ & 21.152 & 0.070924 & 0.00002587 \\
\hline
\end{tabular}

Total energy required to maintain reactor temperature:

$\mathrm{Q}($ Energy $)=\Delta \mathrm{H}$ (heat content of the system)

$\Delta \mathrm{H}=($ Rate of internal enthalpy change + Rate of enthalpy of reaction $)$ - from Chapter 7 of (Morris et al., 2011)

TG + 3 MeOH -> GL + 3FAME (Dong et al., 2016)

Rate of enthalpy of reaction (heat given off or required by/for the reaction):

Enthalpy of reaction $=$ heat of formation of products - heat of formation of reactants

Enthalpy of reaction $=(1 \mathrm{~mol}$ glycerol $)(-669.6 \mathrm{~kJ} / \mathrm{mol}$ glycerol $)+(3$ moles FAME $)(-734.5 \mathrm{~kJ} / \mathrm{mol}$ FAME $)-[(1 \mathrm{~mol} \mathrm{TG})(-2,129.07$ $\mathrm{kJ} / \mathrm{mol} \mathrm{TG})+(3 \mathrm{~mol}$ methanol $)(-239.2 \mathrm{~kJ} / \mathrm{mol}$ methanol $)]$

Enthalpy of reaction $=-26.43 \mathrm{~kJ} / \mathrm{mol}$ (exothermic reaction) 
Extent of enthalpy of reaction $($ rate $)=(0.5 \mathrm{kmol} \mathrm{TG} / \mathrm{hr})(1 \mathrm{hr} / 3600 \mathrm{~s})(-26.43 \mathrm{~kJ} / \mathrm{mol})(1000 \mathrm{~mol} / \mathrm{kmol})=-3.67 \mathrm{~kJ} / \mathrm{s} \mathrm{or}-3.67 \mathrm{~kW}$

Rate of internal enthalpy change (heat required or given off based on the heat capacity of the materials and their correlated temperature changes):

$\mathrm{Q}=\Sigma$ Internal enthalpy rate output $-\Sigma$ Internal enthalpy rate input + rate of enthalpy of reaction

$\Sigma$ Internal enthalpy rate input $=\mathrm{n} 1 \mathrm{H} 1+\mathrm{n} 2 \mathrm{H} 2$

$\Sigma$ Internal enthalpy rate output $=\mathrm{n} 3 \mathrm{H} 3+\mathrm{n} 4 \mathrm{H} 4+\mathrm{n} 6 \mathrm{H} 6+\mathrm{n} 8 \mathrm{H} 8+\mathrm{n} 5 \mathrm{H} 5+\mathrm{n} 7 \mathrm{H} 7$

$\mathrm{H} 1=\int(673 \mathrm{~K}-298 \mathrm{~K}) \mathrm{Cp}(\mathbf{T G}) \mathrm{dT}$

$\mathrm{H} 1=\int(673 \mathrm{~K}-298 \mathrm{~K})\left(\mathrm{Ai}+\mathrm{BiT}+\mathrm{CiT}^{\wedge} 2\right) \mathrm{dT}($ in $\mathrm{J} / \mathrm{kmol} * \mathrm{~K})$

$\mathrm{H} 1=1475.08 \mathrm{~T}-\left(0.71 \mathrm{~T}^{\wedge} 2\right) / 2+\left(5.85 \mathrm{~T}^{\wedge} 3\right) / 3^{*} 10^{\wedge} 3$

$\mathrm{H} 1=1475.08(673-298)-\left(0.71\left(673^{\wedge} 2-298^{\wedge} 2\right)\right) / 2+\left(5.85\left(673^{\wedge} 3-298^{\wedge} 3\right)\right) / 3^{*} 10^{\wedge} 3$

$\mathrm{H} 1=966,688 \mathrm{~J} / \mathrm{kmol}$

$\mathrm{H} 2=\int(338 \mathrm{~K}-298 \mathrm{~K}) \mathrm{Cp}(\mathrm{l})(\mathbf{M e O H}) \mathrm{dT}+\mathbf{\Delta H} \mathbf{v a p}+\int(673 \mathrm{~K}-338 \mathrm{~K}) \mathrm{Cp}(\mathrm{g})(\mathbf{M e O H}) \mathrm{dT}$

Assume $\Delta \mathrm{H}$ vap supersedes

$\Delta \mathrm{H} 2$ vap $=37.6 \mathrm{~kJ} / \mathrm{mol}$

$\mathrm{H} 3=\int(511 \mathrm{~K}-298 \mathrm{~K}) \mathrm{Cp}(\mathrm{l})(\mathbf{M G}) \mathrm{dT}+\mathbf{\Delta H} \mathbf{v a p}+\int(673 \mathrm{~K}-511 \mathrm{~K}) \mathrm{Cp}(\mathrm{g})(\mathbf{M G}) \mathrm{dT}$

Assume $\Delta \mathrm{H}$ vap supersedes

$\Delta \mathrm{H} 3$ vap $=151.86 \mathrm{~kJ} / \mathrm{mol}$ 
$(H 3[511-298]=534,768 \mathrm{~J} / \mathrm{kmol})$

$\mathrm{H} 4=\int(670 \mathrm{~K}-298 \mathrm{~K}) \mathrm{Cp}(1)(\mathbf{D G}) \mathrm{dT}+\Delta \mathbf{H}$ vap

Assume $\Delta H$ vap supersedes

$\Delta \mathrm{H} 4 \mathrm{vap}=222.69 \mathrm{~kJ} / \mathrm{mol}$

$(H 4[670-298]=751,121 \mathrm{~J} / \mathrm{kmol})$

$\mathrm{H} 5=\int(673 \mathrm{~K}-298 \mathrm{~K}) \mathrm{Cp}(\mathbf{T G}) \mathrm{dT}$

$\mathrm{H} 5=966,688 \mathrm{~J} / \mathrm{kmol}$

H6 $=\int(491 \mathrm{~K}-298 \mathrm{~K}) \mathrm{Cp}(\mathbf{F A M E}) \mathrm{dT}+\mathbf{\Delta H} \mathbf{v a p}+\int(673 \mathrm{~K}-491 \mathrm{~K}) \mathrm{Cp}(\mathrm{g})(\mathbf{F A M E}) \mathrm{dT}$ Assume $\Delta \mathrm{H}$ vap supersedes

$\Delta \mathrm{H} 6$ vap $=84.6 \mathrm{~kJ} / \mathrm{mol}$

$(\mathrm{H6}[491-298]=326,656 \mathrm{~J} / \mathrm{kmol})$

$\mathrm{H} 7=\int(338 \mathrm{~K}-298 \mathrm{~K}) \mathrm{Cp}(\mathbf{M e O H}) \mathrm{dT}+\boldsymbol{\Delta} \mathbf{H} \mathbf{v a p}+\int(673 \mathrm{~K}-338 \mathrm{~K}) \mathrm{Cp}(\mathrm{g})(\mathbf{M e O H}) \mathrm{dT}$ Assume $\Delta \mathrm{H}$ vap supersedes

$\Delta \mathrm{H} 7 \mathrm{vap}=37.6 \mathrm{~kJ} / \mathrm{mol}$

$(H 7[338-298]=20,827 \mathrm{~J} / \mathrm{kmol})$ 
$\mathrm{H} 8=\int(561 \mathrm{~K}-298 \mathrm{~K}) \mathrm{Cp}(\mathbf{G l y}) \mathrm{dT}+\Delta \mathbf{H} \mathbf{v a p}+\int(561 \mathrm{~K}-491 \mathrm{~K}) \mathrm{Cp}(\mathrm{g})(\mathbf{G l y}) \mathrm{dT}$

Assume $\Delta \mathrm{H}$ vap supersedes

$\Delta \mathrm{H} 8$ vap $=91.7 \mathrm{~kJ} / \mathrm{mol}$

$(H 8[561-298]=320,028 \mathrm{~J} / \mathrm{kmol})$

$\Sigma$ Internal enthalpy rate input $=\mathrm{n} 1 \cdot \mathrm{H} 1+\mathrm{n} 2 \cdot \mathrm{H} 2$

$\Sigma$ Internal enthalpy rate input $=0.5 \mathrm{kmol}$ lipids $/ \mathrm{hr}(967 \mathrm{~kJ} / \mathrm{kmol})+4.510 \mathrm{kmol}$ methanol $/ \mathrm{hr}(37,006 \mathrm{~kJ} / \mathrm{kmol})$

$\Sigma$ Internal enthalpy rate input $=483.5 \mathrm{~kJ} / \mathrm{hr}+166,527 \mathrm{~kJ} / \mathrm{hr}$

$\Sigma$ Internal enthalpy rate input $=167,011 \mathrm{~kJ} / \mathrm{hr}$

$\Sigma$ Internal enthalpy rate input $=46.4 \mathrm{~kW}$

$\Sigma$ Internal enthalpy rate output $=\mathrm{n} 8 \cdot \mathrm{H} 8+\mathrm{n} 3 \cdot \mathrm{H} 3+\mathrm{n} 4 \cdot \mathrm{H} 4+\mathrm{n} 5 \cdot \mathrm{H} 5+\mathrm{n} 6 \cdot \mathrm{H} 6+\mathrm{n} 7 \cdot \mathrm{H} 7$

$\Sigma$ Internal enthalpy rate output $=0.58 \mathrm{kmol} \mathrm{GLY} / \mathrm{hr}(91,700 \mathrm{~kJ} / \mathrm{kmol})+0.01 \mathrm{kmol} \mathrm{MG} / \mathrm{hr}(151,860 \mathrm{~kJ} / \mathrm{kmol})+0.003 \mathrm{kmol} \mathrm{DG} / \mathrm{hr}$ $(222,690 \mathrm{kJk} / \mathrm{mol})+0.0001 \mathrm{kmol} \mathrm{TG} / \mathrm{hr}(967 \mathrm{~kJ} / \mathrm{kmol})+1.45 \mathrm{kmol} \mathrm{FAME} / \mathrm{hr}(84,600 \mathrm{~kJ} / \mathrm{kmol})+3.05 \mathrm{kmol} \mathrm{MeOH} / \mathrm{hr}(37,600$ $\mathrm{kJ} / \mathrm{kmol}$ )

$\Sigma$ Internal enthalpy rate output $=53,186 \mathrm{~kJ} / \mathrm{hr}+15,186 \mathrm{~kJ} / \mathrm{hr}+668.1 \mathrm{~kJ} / \mathrm{hr}+0.1 \mathrm{~kJ} / \mathrm{hr}+122,670 \mathrm{~kJ} / \mathrm{hr}+114,680 \mathrm{~kJ} / \mathrm{hr}$

$\Sigma$ Internal enthalpy rate output $=306,390 \mathrm{~kJ} / \mathrm{hr}$

$\Sigma$ Internal enthalpy rate output $=85 \mathrm{~kW}$ (products require more energy for the same temperature increase)

$\mathrm{Q}=\Delta \mathrm{H}$

$\mathrm{Q}=\Sigma$ Internal enthalpy rate output $-\Sigma$ Internal enthalpy rate input + rate of enthalpy of reaction 
$\mathrm{Q}=85 \mathrm{~kW}-46.4 \mathrm{~kW}+3.67 \mathrm{~kW}$

$\mathrm{Q}=42.3 \mathrm{~kW}$ (required to maintain reactor temperature)

\section{A.4.3 CLR 01 - Cooler for transesterification products (input\# 13)}

Details/Assumptions:

- Input: $\mathrm{T}_{\text {in }}=400^{\circ} \mathrm{C}, \mathrm{P}_{\text {in }}=15 \mathrm{mPa}$

- Output: $\mathrm{T}_{\text {out }}=65^{*} \mathrm{C}, \mathrm{P}_{\text {out }}=1 \mathrm{~atm}\left(65^{\circ} \mathrm{C}\right.$ boiling point of methanol - Table A.8)

- Heat capacity of Dowtherm A: $1.587 \mathrm{~kJ} / \mathrm{kg} \cdot \mathrm{K}\left(@ 25^{\circ} \mathrm{C}\right)$ - using the same fluid to cool as to heat (The DOW Chemical Company, 1997)

- Note - the pressure drop will decrease the amount of energy required to cool the products. Energy required to cool was over estimated. That amount is estimated to be rather small and inconsequential overall.

\section{Calculations:}

$\mathrm{Q}=\Delta$ Hvap of glycerol, $\mathrm{MG}, \mathrm{DG}$ and FAME as these transition from gas to liquid during cooling

$\mathrm{Q}=(91.7 \mathrm{~kJ} / \mathrm{mol} \cdot 1 \mathrm{~mol} / 0.092 \mathrm{~kg} \cdot 53.34 \mathrm{~kg} / \mathrm{hr})+(151.86 \mathrm{~kJ} / \mathrm{mol} \cdot 1 \mathrm{~mol} / 0.356 \mathrm{~kg} \cdot 5.17 \mathrm{~kg} / \mathrm{hr})+(222.69 \mathrm{~kJ} / \mathrm{mol} \cdot 1 \mathrm{~mol} / 0.621 \mathrm{~kg} \cdot 1.59 \mathrm{~kg} / \mathrm{hr})$ $+(84.6 \mathrm{~kJ} / \mathrm{mol} \cdot 1 \mathrm{~mol} / 0.296 \mathrm{~kg} \cdot 429.53 \mathrm{~kg} / \mathrm{hr})$

$\mathrm{Q}=53,166 \mathrm{~kJ} / \mathrm{hr}+2,205 \mathrm{~kJ} / \mathrm{hr}+570 \mathrm{~kJ} / \mathrm{hr}+122,764 \mathrm{~kJ} / \mathrm{hr}$

$\mathrm{Q}=178,705 \mathrm{~kJ} / \mathrm{hr}$

$\mathrm{Q}=50 \mathrm{~kW}$

A.4.4 EVP 02 - Evaporate methanol (output\# 16)

Details/Assumptions: 
- no energy required to evaporate methanol here as output temperature from cooler (CLR 01) is the evaporation temperature of methanol $\left(65^{\circ} \mathrm{C}\right)$ (Table A.8)

- $\quad 2-5 \%$ methanol remains in BD. Using 3\% methanol remains with oil and is not extracted (Kusdiana \& Saka, 2004).

- $5 \mathrm{wt} \%$ oil of methanol is required to assist with phase separation between glycerol and with oil (Patil et al., 2012). Therefore, 5 wt\% oil of methanol will not be evaporated at EVP 02 and will be evaporated at EVP 03 after glycerol/lipid separation.

Calculations:

$97.70 \mathrm{~kg}$ methanol $/ \mathrm{hr}-(97.70 \mathrm{~kg}$ methanol $/ \mathrm{hr} \cdot 0.03)=94.39 \mathrm{~kg}$ methanol $/ \mathrm{hr}($ recovered $)$

$2.9 \mathrm{~kg}$ methanol$/ \mathrm{hr}$ remains in $\mathrm{BD}$

$0.11 \mathrm{~kg} \mathrm{TG} / \mathrm{hr}+1.59 \mathrm{~kg} \mathrm{DG} / \mathrm{hr}+5.17 \mathrm{~kg} \mathrm{MG} / \mathrm{hr}+429.53 \mathrm{~kg} \mathrm{FAME} / \mathrm{hr}=436.4 \mathrm{~kg} \mathrm{oil} / \mathrm{hr}$

$436.4 \mathrm{~kg}$ oil $/ \mathrm{hr} \cdot 0.05=21.82 \mathrm{~kg}$ of methanol required to remain with oil

$97.70 \mathrm{~kg}$ methanol $/ \mathrm{hr}-21.82 \mathrm{~kg}$ methanol $/ \mathrm{hr}=75.88 \mathrm{~kg}$ methanol $/ \mathrm{hr}$ evaporated

\section{A.4.5 CDN 02 - Condenser for methanol (input \#21, 22, output \#17)}

\section{Details/Assumptions}

- methanol completely condensed from 65 to $25^{\circ} \mathrm{C}$

- water used here for cooling

- this condenser collects all methanol from both EVP 02 and EVP 03 


\section{Calculations:}

Output \#17 - methanol

$97.70 \mathrm{~kg}$ methanol $/ \mathrm{hr}-(97.70 \mathrm{~kg}$ methanol $/ \mathrm{hr} \cdot 0.03)=94.39 \mathrm{~kg}$ methanol $/ \mathrm{hr}($ recovered $)$

Input \#20 - cooling energy

$\mathrm{H} 1=-\int(338 \mathrm{~K}-298 \mathrm{~K}) \mathrm{Cp}(1)(\mathbf{M e O H}) \mathrm{dT}-\mathbf{\Delta H}$ vap

Assume $\Delta \mathrm{H}$ vap supersedes

$\mathrm{H} 1=-37.6 \mathrm{~kJ} / \mathrm{mol}$

$\mathrm{Q}=\Sigma$ Internal enthalpy rate $=\mathrm{n} 1 \mathrm{H} 1$

$\mathrm{Q}=94.39 \mathrm{~kg} / \mathrm{hr} \cdot 1 \mathrm{~mol} / 0.032 \mathrm{~kg} \cdot(-37.6 \mathrm{~kJ} / \mathrm{mol})$

$\mathrm{Q}=-110,908 \mathrm{~kJ} / \mathrm{hr}$

$\mathrm{Q}=30.8 \mathrm{~kW}$

Input \#22

Total amount of methanol required to be sourced (because of recovery)

$144.25 \mathrm{~kg}$ methanol/ $\mathrm{hr}-94.39 \mathrm{~kg}$ methanol $/ \mathrm{hr}$ recovered $=49.86 \mathrm{~kg}$ methanol required

A.4.6 CEN 03 - Centrifuge to separate glycerol and lipids (input \#29, output\#19)

Details/Assumptions:

- $\quad$ MAC 203 disk bowl centrifuge used here (1HP = 0.746 kW) (United States of America Centrifuge Systems, 2017) 


\section{Calculations:}

Output \#19 - methanol

$97.70 \mathrm{~kg}$ methanol $/ \mathrm{hr} \cdot 0.03=2.9 \mathrm{~kg}$ methanol $/ \mathrm{hr}$ remains in $\mathrm{BD}$

$\underline{\text { Input } \# 29 \text { - centrifuge power requirement }}$

$0.746 \mathrm{kWh}$ (for 1 hour)

A.4.7 EVP 03 - Evaporate rest of methanol (input \#32, output \#24)

Details/Assumptions:

- Using NG for heat source

Calculations:

Output \#24 - methanol

$21.82 \mathrm{~kg}$ methanol $/ \mathrm{hr}-2.9 \mathrm{~kg}$ methanol $/ \mathrm{hr}=18.92 \mathrm{~kg}$ methanol $/ \mathrm{hr}$

Input \#32- heat energy

$\mathrm{H} 1=\int(338 \mathrm{~K}-298 \mathrm{~K}) \mathrm{Cp}(\mathrm{l})(\mathbf{M e O H}) \mathrm{dT}+\Delta \mathbf{H}$ vap

Assume $\Delta \mathrm{H}$ vap supersedes

$\mathrm{H} 1=37.6 \mathrm{~kJ} / \mathrm{mol}$

$\mathrm{H} 2=\int(338 \mathrm{~K}-298 \mathrm{~K}) \mathrm{Cp}(\mathrm{l})(\mathbf{G L Y}) \mathrm{dT}$ - assume negligible compared to $\Delta \mathrm{H}_{\text {vap }}$ above 
$\mathrm{Q}=\Sigma$ Internal enthalpy rate $=\mathrm{n} 1 \cdot \mathrm{H} 1+\mathrm{n} 2 \cdot \mathrm{H} 2$

$\mathrm{Q}=18.92 \mathrm{~kg} / \mathrm{hr} \cdot 1 \mathrm{~mol} / 0.032 \mathrm{~kg} \cdot 37.6 \mathrm{~kJ} / \mathrm{mol}$

$\mathrm{Q}=22,231 \mathrm{~kJ} / \mathrm{hr}$

\section{A.4.8 EVP 04 - Evaporate BD (input\# 38)}

Details/Assumptions:

- $\quad$ Evaporating BD from the other lipids $\left(50^{\circ} \mathrm{C}-218^{\circ} \mathrm{C}\right)$ (see Table A.8 for reference)

- Using NG for heat source and Dowthern A for refrigerant medium

- Dowthern A - used in liquid phase heat exchanger from $15-400^{\circ} \mathrm{C}$ (can be used as heat exchanger fluid in vapour form from $257^{\circ} \mathrm{C}$ to $\left.400^{\circ} \mathrm{C}\right)$ and freezing point $\left(12^{\circ} \mathrm{C}\right)$ (The DOW Chemical Company, 1997)

- Molecular weight of Dowthern A: 166 g/mol (The DOW Chemical Company, 1997)

- Heat capacity of Dowtherm A: $2.162 \mathrm{~kJ} / \mathrm{kg} \cdot \mathrm{K}$ (@230² ) (The DOW Chemical Company, 1997)

- Heat for Downthern A (Q) is from NG in GaBi using LHV of NG

Table A.12: Parameters associated with the evaporation of BD (FAME) from residue lipids

\begin{tabular}{|l|l|l|l|l|}
\hline Product & $\begin{array}{l}\text { Mass flow rate } \\
(\mathbf{k g} / \mathbf{h r})\end{array}$ & $\begin{array}{l}\text { Molar flow rate } \\
\mathbf{( k m o l} / \mathbf{h r})\end{array}$ & $\begin{array}{l}\text { Internal Enthalpy } \\
\text { designate }\end{array}$ & $\begin{array}{l}\text { Internal Enthalpy value } \\
(\mathbf{k J} / \mathbf{m o l})\end{array}$ \\
\hline Monoglyceride & 5.17 & 0.01 & $\mathrm{H} 1$ & $0.38 \mathrm{~kJ} / \mathrm{mol}$ \\
\hline Diglyceride & 1.59 & 0.003 & $\mathrm{H} 2$ & $0.42 \mathrm{~kJ} / \mathrm{mol}$ \\
\hline Triglyceride & 0.11 & 0.0001 & $\mathrm{H} 3$ & $0.7 \mathrm{~kJ} / \mathrm{mol}$ \\
\hline FAME & 429.53 & 1.45 & $\mathrm{H} 4$ & $84.71 \mathrm{~kJ} / \mathrm{mol}$ \\
\hline Methanol & 97.70 & 3.05 & $\mathrm{H} 5$ & $45.96 \mathrm{~kJ} / \mathrm{mol}$ \\
\hline
\end{tabular}




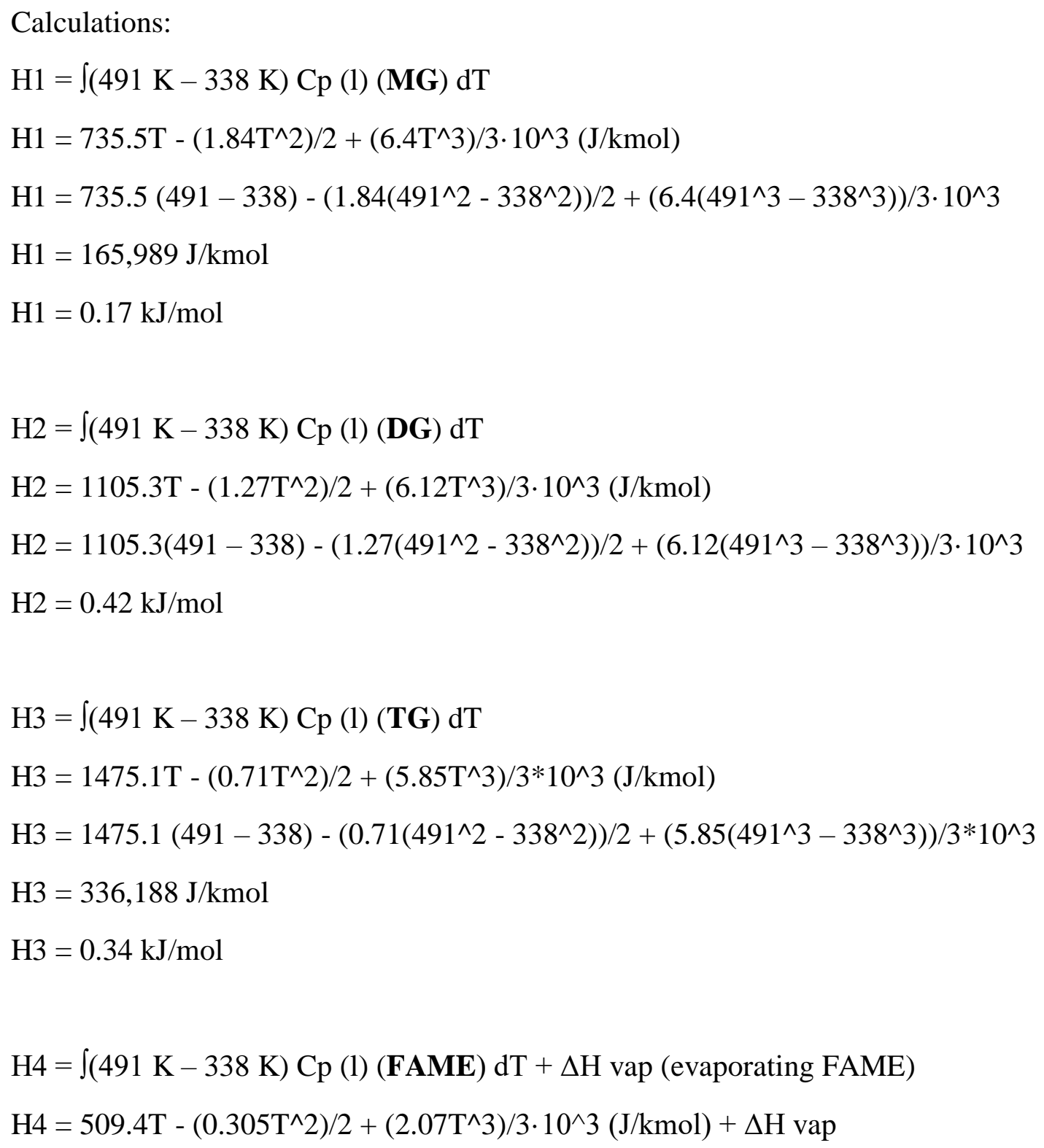




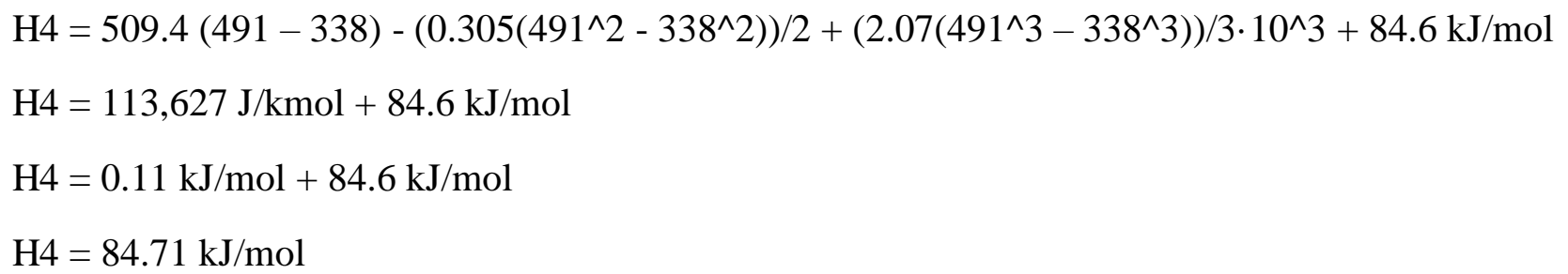


- $\quad \mathrm{Q}=\Sigma \mathrm{niHi}$

- $\quad \mathrm{T}_{\text {in }}=218^{\circ} \mathrm{C}, \mathrm{T}_{\text {out }}=25^{\circ} \mathrm{C}$

- Dowthern A used here to cool

Table A.13: Properties of BD stream for cooling purposes

\begin{tabular}{|l|l|l|l|l|}
\hline Component & Mass flow rate (kg/hr) & $\begin{array}{l}\text { Molar flow rate } \\
\text { (mol/hr) }\end{array}$ & Enthalpy designate & Enthalpy (kJ/mol) \\
\hline FAME & 429.53 & $1,448.67$ & $\mathrm{H} 1$ & $-84.7 \mathrm{~kJ} / \mathrm{mol}$ \\
\hline Methanol & 3.03 & 94.69 & $\mathrm{H} 2$ & $-37.6 \mathrm{~kJ} / \mathrm{mol}$ \\
\hline
\end{tabular}

Calculations:

$\mathrm{H} 1=\int(298 \mathrm{~K}-491 \mathrm{~K}) \mathrm{Cp}(\mathrm{l})(\mathbf{F A M E}) \mathrm{dT}-\Delta \mathrm{H}$ vap (condensing FAME)

$\mathrm{H} 1=509.41 \mathrm{~T}-\left(0.3055 \mathrm{~T}^{\wedge} 2\right) / 2+\left(2.069 \mathrm{~T}^{\wedge} 3\right) / 3 \cdot 10^{\wedge} 3(\mathrm{~J} / \mathrm{kmol})-\Delta \mathrm{H}$ vap

$\mathrm{H} 1=509.41(298-491)-\left(0.3055\left(298^{\wedge} 2-491^{\wedge} 2\right)\right) / 2+\left(2.069\left(491^{\wedge} 3-338^{\wedge} 3\right)\right) / 3 \cdot 10^{\wedge} 3-84.6 \mathrm{~kJ} / \mathrm{mol}$

$\mathrm{H} 1=-0.138 \mathrm{~kJ} / \mathrm{mol}-84.6 \mathrm{~kJ} / \mathrm{mol}$

$\mathrm{H} 1=-84.7 \mathrm{~kJ} / \mathrm{mol}$

$\mathrm{H} 2=-\left[\int(338 \mathrm{~K}-491 \mathrm{~K}) \mathrm{Cp}(\mathrm{g})(\mathbf{M e O H}) \mathrm{dT}+\Delta \mathrm{H}\right.$ vaporization (condensing methanol) $\left.+\int(298 \mathrm{~K}-338 \mathrm{~K}) \mathrm{Cp}(\mathrm{l})(\mathbf{M e O H}) \mathrm{dT}\right]$ *assume $\Delta$ Hvap supersedes

$\mathrm{H} 2=-37.6 \mathrm{~kJ} / \mathrm{mol}$

$\mathrm{Q}($ Energy $)=\mathrm{n} 1 \cdot \mathrm{H} 1+\mathrm{n} 2 \cdot \mathrm{H} 2($ no reaction $)$

$\mathrm{Q}($ Energy $)=1,448.67 \mathrm{~mol} / \mathrm{hr}(-84.7 \mathrm{~kJ} / \mathrm{mol})+94.69 \mathrm{~mol} / \mathrm{hr}(-37.6 \mathrm{~kJ} / \mathrm{mol})$ 
$\mathrm{Q}=-122,702 \mathrm{~kJ} / \mathrm{hr}-3,560 \mathrm{~kJ} / \mathrm{hr}$

$\mathrm{Q}=-126,262 \mathrm{~kJ} / \mathrm{hr}$

$Q=35$ kW

A.5 IO for ABE production

Table A.14: ABE process input/output table

\begin{tabular}{|c|c|c|c|c|c|c|}
\hline Stage & & Inputs & Input amount & & Outputs & Output amount \\
\hline $\begin{array}{l}\text { Pre-treat for } \\
\text { Ligno/Protein } \\
\text { stream (MXT } \\
01)\end{array}$ & $\begin{array}{l}1 \\
2 \\
3 \\
4\end{array}$ & $\begin{array}{l}\text { Lignocellulosic } \\
\text { Protein } \\
\mathrm{H}_{2} \mathrm{SO}_{4}(1 \%) \\
\text { Water }\end{array}$ & $\begin{array}{l}443.26 \mathrm{~kg} / \mathrm{hr} \\
\mathbf{3 7 9 . 9 4} \mathrm{kg} / \mathrm{hr} \\
175 \mathrm{~kg} / \mathrm{hr} \\
9,573.8 \mathrm{~kg} / \mathrm{hr}\end{array}$ & $\begin{array}{l}1 \\
2 \\
3\end{array}$ & $\begin{array}{l}\text { Lignocellulosic } \\
\text { Protein } \\
\text { Dilute sulfuric acid } \\
\text { solution }\end{array}$ & $\begin{array}{l}443.26 \mathrm{~kg} / \mathrm{hr} \\
\mathbf{3 7 9 . 9 4} \mathrm{kg} / \mathrm{hr} \\
9,573.8 \mathrm{~kg} / \mathrm{hr}\end{array}$ \\
\hline $\begin{array}{l}\text { Heated vessel } \\
\text { (STX 01) }\end{array}$ & $\begin{array}{l}5 \\
6 \\
7\end{array}$ & $\begin{array}{l}\text { Ligno + protein } \\
\mathrm{H}_{2} \mathrm{SO}_{4} \text { solution } \\
\text { Heat (Q) }\end{array}$ & $\begin{array}{l}823.2 \mathrm{~kg} / \mathrm{hr} \\
9,573.8 \mathrm{~kg} / \mathrm{hr} \\
4,176 \mathrm{MJ} / \mathrm{hr}\end{array}$ & $\begin{array}{l}4 \\
5 \\
6 \\
7\end{array}$ & $\begin{array}{l}\mathrm{H}_{2} \mathrm{SO}_{4} \text { solution } \\
\text { Sugar } \\
\text { LO ligno } \\
\text { Protein }\end{array}$ & $\begin{array}{l}\text { 9,573.8 kg/hr } \\
\mathbf{4 2 1 . 1 0 ~ k g / h r} \\
22.163 \mathrm{~kg} / \mathrm{hr} \\
379.94 \mathrm{~kg} / \mathrm{hr}\end{array}$ \\
\hline $\begin{array}{l}\text { Cooler (CLR } \\
02)\end{array}$ & $\begin{array}{l}8 \\
9 \\
10 \\
11 \\
12\end{array}$ & $\begin{array}{l}\mathrm{H}_{2} \mathrm{SO}_{4} \text { solution } \\
\text { Sugar } \\
\text { LO ligno } \\
\text { Protein } \\
\text { Cooling }(\mathrm{Q})\end{array}$ & $\begin{array}{l}9,573.8 \mathrm{~kg} / \mathrm{hr} \\
\mathbf{4 2 1 . 1 0} \mathbf{~ k g} / \mathrm{hr} \\
22.163 \mathrm{~kg} / \mathrm{hr} \\
\mathbf{3 7 9 . 9 4} \mathbf{~ k g} / \mathrm{hr} \\
-3,306 \mathrm{MJ} / \mathrm{hr}\end{array}$ & $\begin{array}{l}8 \\
9 \\
10 \\
11\end{array}$ & $\begin{array}{l}\mathrm{H}_{2} \mathrm{SO}_{4} \text { solution } \\
\text { Sugar } \\
\text { LO ligno } \\
\text { Protein }\end{array}$ & $\begin{array}{l}\text { 9,573.8 kg/hr } \\
\mathbf{4 2 1 . 1 0 ~ k g / h r} \\
22.163 \mathrm{~kg} / \mathrm{hr} \\
\mathbf{3 7 9 . 9 4} \mathbf{~ k g} / \mathrm{hr}\end{array}$ \\
\hline $\begin{array}{l}\text { Neutralizatio } \\
\text { n (MXT 02) }\end{array}$ & $\begin{array}{l}13 \\
14 \\
15 \\
16\end{array}$ & $\begin{array}{l}\text { Sugar } \\
\mathrm{NaOH} \\
\mathrm{H}_{2} \mathrm{SO}_{4} \text { solution } \\
\mathrm{LO} \text { lingo + protein }\end{array}$ & $\begin{array}{l}421.10 \mathrm{~kg} / \mathrm{hr} \\
\mathbf{1 6 5} \mathbf{~ k g} / \mathbf{h r} \\
9,573.8 \mathrm{~kg} / \mathrm{hr} \\
\mathbf{4 0 2 . 1 0} \mathbf{~ k g} / \mathbf{h r}\end{array}$ & $\begin{array}{l}12 \\
13 \\
14 \\
15 \\
16\end{array}$ & $\begin{array}{l}\text { Sugar } \\
\mathrm{NaOH} \\
\mathrm{H}_{2} \mathrm{SO}_{4} \\
\text { Wastewater } \\
\text { LO lingo + protein }\end{array}$ & $\begin{array}{l}421.10 \mathrm{~kg} / \mathrm{hr} \\
\mathbf{0} \mathrm{kg} / \mathrm{hr} \\
0 \mathrm{~kg} / \mathrm{hr} \\
\mathbf{9 , 6 4 7 . 8 ~ k g / h r} \\
402.10 \mathrm{~kg} / \mathrm{hr}\end{array}$ \\
\hline
\end{tabular}




\begin{tabular}{|c|c|c|c|c|c|c|}
\hline $\begin{array}{l}\text { Airlift } \\
\text { bioreactor } \\
\text { (FRM 01) }\end{array}$ & $\begin{array}{l}17 \\
18 \\
19 \\
20 \\
21 \\
22 \\
23 \\
\\
24\end{array}$ & $\begin{array}{l}\text { Sugar } \\
\text { LO lingo + protein } \\
\text { Nitrogen } \\
\text { Bacteria } \\
\text { Nutrient medium } \\
\text { Wastewater } \\
\text { Energy required for } \\
\text { reflux of nitrogen } \\
\text { Energy required for } \\
\text { stripping nitrogen }\end{array}$ & 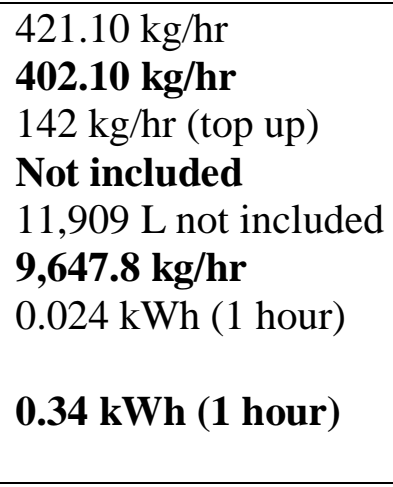 & $\begin{array}{l}17 \\
18 \\
19 \\
20 \\
21 \\
22 \\
23\end{array}$ & $\begin{array}{l}\text { Bio-acetone } \\
\text { Bio-butanol } \\
\text { Bio-ethanol } \\
\text { Wastewater } \\
\text { Protein + LO ligno } \\
\text { UR lingo } \\
\mathrm{CO}_{2}\end{array}$ & 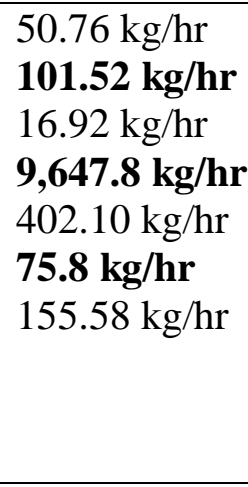 \\
\hline $\begin{array}{l}\text { Bio-acetone } \\
\text { Evaporator } \\
\text { (EVP 05) }\end{array}$ & $\begin{array}{l}25 \\
26 \\
27 \\
28 \\
29 \\
30 \\
31\end{array}$ & $\begin{array}{l}\text { Bio-acetone } \\
\text { Bio-butanol } \\
\text { Bio-ethanol } \\
\text { Water } \\
\text { Prevaporator water flow } \\
\text { energy } \\
\text { Chitosan membrane } \\
\text { Heat (Q) }\end{array}$ & $\begin{array}{l}50.76 \mathrm{~kg} / \mathrm{hr} \\
\mathbf{1 0 1 . 5 2} \mathbf{~ k g} / \mathrm{hr} \\
16.92 \mathrm{~kg} / \mathrm{hr} \\
\mathbf{1 7 4} \mathbf{~ k g} / \mathbf{h r} \\
6.4 \text { x10-3kW (over } 1 \\
\text { hour) } \\
\mathbf{2 . 3} \mathbf{~ m e m b r a n e s ~ p e r ~} \\
\text { year } \\
13.94 \mathrm{kWh} \text { (EVP 05 } \\
\text { and EVP 06 energy) }\end{array}$ & $\begin{array}{l}24 \\
25 \\
26\end{array}$ & $\begin{array}{l}\text { Bio-acetone } \\
\text { Bio-butanol } \\
\text { Bio-ethanol }\end{array}$ & $\begin{array}{l}50.76 \mathrm{~kg} / \mathrm{hr} \\
101.52 \mathrm{~kg} / \mathrm{hr} \\
16.92 \mathrm{~kg} / \mathrm{hr}\end{array}$ \\
\hline $\begin{array}{l}\text { Bio-acetone } \\
\text { Condenser } \\
(\text { CND 04) }\end{array}$ & $\begin{array}{l}32 \\
33\end{array}$ & $\begin{array}{l}\text { Bio-acetone } \\
\text { Cooling energy }\end{array}$ & $50.76 \mathrm{~kg} / \mathrm{hr}$ & 27 & Bio-acetone & $50.76 \mathrm{~kg} / \mathrm{hr}$ \\
\hline $\begin{array}{l}\text { Bio-ethanol } \\
\text { Evaporator } \\
\text { (EVP 06) }\end{array}$ & $\begin{array}{l}35 \\
36\end{array}$ & $\begin{array}{l}\text { Bio-butanol } \\
\text { Bio-ethanol }\end{array}$ & $\begin{array}{l}101.52 \mathrm{~kg} / \mathrm{hr} \\
16.92 \mathrm{~kg} / \mathrm{hr}\end{array}$ & $\begin{array}{l}28 \\
29\end{array}$ & $\begin{array}{l}\text { Bio-butanol } \\
\text { Bio-ethanol }\end{array}$ & $\begin{array}{l}101.52 \mathrm{~kg} / \mathrm{hr} \\
16.92 \mathrm{~kg} / \mathrm{hr}\end{array}$ \\
\hline $\begin{array}{l}\text { Bio-ethanol } \\
\text { Condenser } \\
(\text { CND 05) }\end{array}$ & $\begin{array}{l}37 \\
38\end{array}$ & $\begin{array}{l}\text { Bio-ethanol } \\
\text { Cooling energy }\end{array}$ & $16.92 \mathrm{~kg} / \mathrm{hr}$ & 30 & Bio-ethanol & $16.92 \mathrm{~kg} / \mathrm{hr}$ \\
\hline
\end{tabular}


A.5.1 MXT 01 - Mixing sulfuric acid for pre-treatment of lignocellulosic \& protein biomass (input \#1, 2, 3, 4)

Details/Assumptions:

- $\quad$ pre-treatment of lignocellulosic biomass with $\mathrm{H}_{2} \mathrm{SO}_{4}$ (Dong et al., 2016)

- Of the 10,000,000 microalgal biomass, 35\% lingnocellulosic biomass and 30\% protein (reasonable based on Table 2.4)

Calculations:

Input \#1,2 - lignocellulosic and protein biomass

$10,000,000 \mathrm{~kg}$ microalgal biomass/year $\cdot(0.35$ lignocellulosic biomass $) / 47$ weeks/year/7 days/week $/ 24$ hours $/$ day $=443.2 \mathrm{~kg}$ lignocellulosic biomass/hr

$10,000,000 \mathrm{~kg}$ microalgal biomass/year $\cdot(0.30$ protein biomass $) / 47$ weeks/year/7 days $/$ week $/ 24$ hours $/$ day $=379.94 \mathrm{~kg}$ protein biomass/hr

\section{$\underline{\text { Input \#3 - sulfuric acid }}$}

$4.3 \mathrm{~g}$ biomass/0.05 L 1\% dilute $\mathrm{H}_{2} \mathrm{SO}_{4}$ solution = $86 \mathrm{~g}$ biomass/L $1 \%$ dilute $\mathrm{H}_{2} \mathrm{SO}_{4}$ solution (Begum \& Dahman, 2015)

Which is $0.01163 \mathrm{~L} 1 \%$ dilute $\mathrm{H}_{2} \mathrm{SO}_{4}$ solution/g biomass $=11.63 \mathrm{~L} 1 \%$ dilute $\mathrm{H}_{2} \mathrm{SO}_{4}$ solution $/ \mathrm{kg}$ biomass

$823.2 \mathrm{~kg}$ biomass $/ \mathrm{hr} \cdot 11.63 \mathrm{~L} 1 \%$ dilute $\mathrm{H}_{2} \mathrm{SO}_{4}$ solution $/ \mathrm{kg}$ biomass $=9,573.82 \mathrm{~L}$ of $1 \%$ dilute $\mathrm{H}_{2} \mathrm{SO}_{4}$ solution $/ \mathrm{hr}$ 9,573.82 $\mathrm{L}$ of $1 \%$ dilute $\mathrm{H}_{2} \mathrm{SO}_{4}$ solution /hr (assuming $1 \%$ volume) $=95.7 \mathrm{~L} \mathrm{H}_{2} \mathrm{SO}_{4} / \mathrm{hr}$

$95.7 \mathrm{~L} \mathrm{H}_{2} \mathrm{SO}_{4} / \mathrm{hr} \cdot 1 \mathrm{~cm}^{3} / 0.001 \mathrm{~L}=95,400 \mathrm{~cm}^{3} / \mathrm{hr} \cdot 1.84 \mathrm{~g} / \mathrm{cm}^{3}$ (density of $\mathrm{H}_{2} \mathrm{SO}_{4}$ ) $=175,535 \mathrm{~g} / \mathrm{hr}$

$\mathrm{pH}$ of solution:

$175.5 \mathrm{~kg} \mathrm{H}_{2} \mathrm{SO}_{4} / 0.098 \mathrm{~kg} / \mathrm{mol}=1,785.7 \mathrm{~mol} \mathrm{H}_{2} \mathrm{SO}_{4}$ in $9,573.8 \mathrm{~L}$ 
$1,785.7 \mathrm{~mol} / 9,573.8 \mathrm{~L}=0.186 \mathrm{~mol} / \mathrm{L}$

$\mathrm{H}_{2} \mathrm{SO}_{4} \rightarrow 2 \mathrm{H}^{+}+\mathrm{SO}_{4}^{-2}$

$0.186 \mathrm{~mol} / \mathrm{L} \mathrm{H}_{2} \mathrm{SO}_{4}=2 \cdot 0.186 \mathrm{~mol} / \mathrm{L}=0.372 \mathrm{~mol} \mathrm{H}+/ \mathrm{L}$

$\mathrm{pH}=-\log _{10}[0.372 \mathrm{~mol} / \mathrm{L}]$

$\mathrm{pH}=0.43$

Input \#4 - water

11.63 L/kg biomass $\cdot 823 \mathrm{~kg}$ biomass $/ \mathrm{hr}=9,573.8 \mathrm{~L}$ of water $/ \mathrm{hr}$ (also 9,573.8 $\mathrm{kg}$ of water $/ \mathrm{hr}$ given $1 \mathrm{~L}$ water $=1 \mathrm{~kg}$ water and the solution is mostly water)

A.5.2 STX 01 - Heating of lignocellulosic and protein biomass (input \#7, output \#5, 6)

Details/Assumptions:

- Heat biomass from $25^{\circ} \mathrm{C}$ to $121^{\circ} \mathrm{C}$ and maintain for 60 mins (Begum \& Dahman 2015)

- Density of water: $1000 \mathrm{~kg} / \mathrm{m}^{3}$, mass of water: $1 \mathrm{~L}=1 \mathrm{~kg}$

- By definition: A pump is a device that moves fluid (either liquid or gas) from one place to another. A compressor is a device that squeezes a gas into a smaller volume and often "pumps" it somewhere else at the same time.

- Density of $1 \%$ sulfuric acid solution: 1.0038 kg/L (Green \& Perry, 2007)

- $\quad$ NG used as heat source while Dowthern A used as refrigerant medium

Calculations:

$\underline{\text { Input \#7 - energy required to heat solution }}$ 
Using the heat capacity of water to find heat required:

$\mathrm{Q}=\mathrm{mCp} \Delta \mathrm{T}$

$\mathrm{Q}=(9,573.8 \mathrm{~kg}$ water $/ \mathrm{hr}+823.2 \mathrm{~kg}$ lignocellulosic biomass and protein $/ \mathrm{hr})(4.184 \mathrm{~kJ} / \mathrm{kg} \cdot \mathrm{K})(394-298) \mathrm{K}$

$\mathrm{Q}=4,176,101 \mathrm{~kJ} / \mathrm{hr}$

$\mathrm{Q}=4,176 \mathrm{MJ} / \mathrm{hr}$

\section{Output \#5, 6-fermentable sugar, LO lignocellulosic biomass}

Amount of lignocellulosic biomass:

$10,000,000 \mathrm{~kg} * 0.35 / 47$ week/year $/ 7$ days/week $/ 24 \mathrm{hr} / \mathrm{day}=443.26 \mathrm{~kg} / \mathrm{hr}$

$443.26 \mathrm{~kg} / \mathrm{hr}$ lignocellulosic biomass $0.95 \%$ released $=421.10 \mathrm{~kg}$ fermentable sugar $/ \mathrm{hr}$

Unreleased lignocellulosic biomass:

$443.26 \mathrm{~kg} / \mathrm{hr}$ lignocellulosic biomass $\cdot 0.05 \%$ released $=22.16 \mathrm{~kg}$ left over (LO) ligncellulosic bimoass $/ \mathrm{hr}$

\section{A.5.3 CLR 02 - Cool after pre-treatment (input \#12)}

Details/Assumptions:

- $\mathrm{Cool}$ from $121^{\circ} \mathrm{C}$ to $45^{\circ} \mathrm{C}$ prior to neutralization. $45^{\circ} \mathrm{C}$ is required for $\mathrm{SSF}$ process

- Dowthern A used here to cool

Calculations:

Amount of energy required to reduce temperature of solution (using water heat capacity):

$\mathrm{Q}=\mathrm{mCp} \Delta \mathrm{T}$ 
$\mathrm{Q}=(9,573.8 \mathrm{~kg}$ water $/ \mathrm{hr}+823.2 \mathrm{~kg}$ lignocellulosic biomass and protein $/ \mathrm{hr})(4.184 \mathrm{~kJ} / \mathrm{kg} \cdot \mathrm{K})(394-318) \mathrm{K}$

$\mathrm{Q}=3,306,080 \mathrm{~kJ} / \mathrm{hr}$

$\mathrm{Q}=3,306 \mathrm{MJ} / \mathrm{hr}$

\section{A.5.4 MXT 02 - Neutralization tank (input \#14, 16, output \#15)}

Details/Assumptions:

- $\quad$ Assume requied to attain $\mathrm{pH}$ of 5 for effective neutralization

- Assume water will be produced from the neutralization reaction and will become wastewater

Input \#14 - sodium hydroxide

To get to $\mathrm{pH}$ of 5 :

$0.43 \mathrm{~mol} / \mathrm{L} \rightarrow 0.00001 \mathrm{~mol} / \mathrm{L}$ requies reduction of $0.42999 \mathrm{~mol} / \mathrm{L}$ of protons

$0.42999 \mathrm{~mol}$ protons $/ \mathrm{L} \cdot 9,573.82 \mathrm{~L}_{\text {of }} \mathrm{H}_{2} \mathrm{SO}_{4}$ solution $/ \mathrm{hr}=4,117 \mathrm{~mol}$ protons to be removed/hr

Need $1 / 2 \mathrm{~mol} \mathrm{H}_{2} \mathrm{SO}_{4}$ per mol of protons according to:

$\mathrm{H}_{2} \mathrm{SO}_{4} \rightarrow 2 \mathrm{H}^{+}+\mathrm{SO}_{4}^{-2}\left(1 \mathrm{~mol}\right.$ of $\mathrm{H}_{2} \mathrm{SO}_{4}$ is 2 mols of $\left.\mathrm{H}^{+}\right)$

$1 / 2 \mathrm{~mol} \mathrm{H}_{2} \mathrm{SO}_{4}$ is needs $1 \mathrm{~mol}$ of $\mathrm{NaOH}$ according to:

$\mathrm{H}_{2} \mathrm{SO}_{4}+2 \mathrm{NaOH} \rightarrow 2 \mathrm{H}_{2} \mathrm{O}+\mathrm{Na}_{2} \mathrm{SO}_{4}$

Therefore, need 4,117 mol of $\mathrm{NaOH}$

$4,117 \mathrm{~mol} \mathrm{NaOH} / \mathrm{hr} \cdot 0.04 \mathrm{~kg} \mathrm{NaOH} / \mathrm{mol}=165 \mathrm{~kg} \mathrm{NaOH} / \mathrm{hr}$ 
Input \#16 - LO lignocellulosic and protein biomass

$379.94 \mathrm{~kg}$ protein $/ \mathrm{hr}+22.16 \mathrm{~kg}$ LO lignocellulosic biomass $=402.10 \mathrm{~kg}$ LO lignocellulosic biomass + protein $/ \mathrm{hr}$

\section{Output \#15 - wastewaster}

Extra water produced:

$4,117 \mathrm{~mol} \mathrm{NaOH} / \mathrm{hr}$ is the same number of mols of water produced according to:

$\mathrm{H}_{2} \mathrm{SO}_{4}+2 \mathrm{NaOH} \rightarrow 2 \mathrm{H}_{2} \mathrm{O}+\mathrm{Na}_{2} \mathrm{SO}_{4}$

4,117 mol water produced $0.018 \mathrm{~kg}$ water $/ \mathrm{mol}=74 \mathrm{~kg}$ water produced $/ \mathrm{hr}$

Total water output:

9,573.8 $\mathrm{kg} \mathrm{H}_{2} \mathrm{SO}_{4}$ solution $/ \mathrm{hr}+(74 \mathrm{~kg}$ water produced $/ \mathrm{hr})=9,647.8 \mathrm{~kg} / \mathrm{hr}$

A.5.5 FRM 01 - Airlift Fermentation Reactor to produce alcohols (input \#19, 23, 24, 20, 21, output \#17, 18, 19, 22, 23)

Details/Assumptions:

- $\quad 82 \%$ of fermentable lignocellulosic biomass fermented (Begum \& Dahman, 2015)

- $\quad 49 \%$ of the fermented sugars converted into ABE products (Begum \& Dahman, 2015)

- $\quad 0.49 \mathrm{~g} \mathrm{ABE} / \mathrm{g}$ fermented sugar - Table 7 in reference (Begum \& Dahman, 2015)

- $\quad$ ABE is produced in ratios of 3:6:1 respectively (Ellis et al., 2012)

- Using volumetric flow rate for nitrogen gas $\left(0.45987 \mathrm{~m}^{3} / \mathrm{s}\right)$ based on sized airlift bioreactor for a similar process with similar volumes (Giang et al. 2017)

- Assume $10 \%$ loss of nitrogen gas during operations

- Density of nitrogen gas $=1.165 \mathrm{~kg} / \mathrm{m}^{3}$ (Green \& Perry, 2007) 
- Density of liquid nitrogen: $808.5 \mathrm{~kg} / \mathrm{m}^{3}$ (Green \& Perry, 2007)

- Only liquid oxygen, and not liquid nitrogen storage and handling, is already practiced by wastewater treatment plants as part of the treatment process (Barrie wastewater treatment facility, 2017). Infrastructure for liquid nitrogen would need to be developed/contracted.

- Assume that flue gas from fermenter is stripped of $\mathrm{N}_{2}$ via filters. Assume power requirements to strip are the same as those for stripping biogas (stripping energy requirements range from 0.15 to $0.5 \mathrm{kWh} / \mathrm{Nm}^{3}$ ( $\mathrm{N}$ refers to the temperature measurement at STP) (Bauer et al., 2013)).

- Assume energy requirements for recirculation of nitrogen is similar to the DAF unit that also re-circulates nitrogen gas

- Wastewater here (with proteins and left over lignocellulosic biomass) will move to the anaerobic digester to produce biosolids for nutrient recovery (see Figure $3.3 \& 3.4$ )

Calculations:

Input \#21, 23, 24 - nitrogen, re-circulation \& strip

Nitrogen required $\left(\mathrm{N}_{2}\right)=0.45987 \mathrm{~m}^{3} / \mathrm{s} \cdot 3600 \mathrm{~s} / \mathrm{hr} / 1.165 \mathrm{~kg} / \mathrm{m}^{3}=1,421.05 \mathrm{~kg} / \mathrm{hr}$

$1,421.05 \mathrm{~kg} / \mathrm{hr} \cdot 0.1=142 \mathrm{~kg} / \mathrm{hr}$

Total amount of $\mathrm{N}_{2}$ required on an hourly bases $=142 \mathrm{~kg} / \mathrm{hr}$

Volume of liquid nitrogen required to make up for loss: $142 \mathrm{~kg} / \mathrm{hr} / 808.5 \mathrm{~kg} / \mathrm{m}^{3}=0.17 \mathrm{~m}^{3}$

Volume of liquid nitrogen re-circulated: $1,421.05 \mathrm{~kg} / \mathrm{hr} / 808.5 \mathrm{~kg} / \mathrm{m}^{3}=1.7 \mathrm{~m}^{3} / \mathrm{hr}$

Power requiremenst for re-circulations $=0.015 \mathrm{kWh} / \mathrm{m}^{3} \cdot 1.7 \mathrm{~m}^{3} / \mathrm{hr}=0.024 \mathrm{kWh}(1$ hour $)$

Stripping power: $0.2 \mathrm{kWh} / \mathrm{m}^{3} \cdot 1.7 \mathrm{~m}^{3} / \mathrm{hr}=0.34 \mathrm{kWh}(1$ hour $)$

$\underline{\text { Input \#20, } 21 \text { - bacteria and nutrient medium }}$ 
Details/Assumptions:

- $250 \mathrm{~mL}$ vessel with $59.59 \mathrm{~g} / \mathrm{L}$ fermentable sugar (Begum \& Dahman, 2015)

- Used $7 \mathrm{~mL}$ bacteria and $40 \mathrm{~mL}$ nutrient medium in the $250 \mathrm{~mL}$ vessel (Begum \& Dahman, 2015)

- Given that it takes 5 days to get full ABE return from a quantity of fermentable sugar, it is best to have 5 days of nutrient medium to maintain bacteria culture long enough for the bacteria culture to transfer from relying on the medium to relying on the algal sugar

- Bacteria Culture: Fused Clostridium beijerinckii (ATCC BA101) and Clostridium thermocellum (ATCC 27405) (Syed, 2012)

- Did not calculate amount of bacteria or water associated with this culture. Based on Syed (2012), these bacteria would be used continuously to produce $\mathrm{ABE}$ and would need to be replaced potentially on a weekly bases. Regardless, this input could not be captured in $\mathrm{GaBi}$ for analysis

\section{Calculations:}

$0.25 \mathrm{~L} \cdot 59.59 \mathrm{~g} / \mathrm{L}=0.0149 \mathrm{~kg}$ sugar in the $250 \mathrm{~mL}$ vessel

Given $345.3 \mathrm{~kg}$ of fermentable sugar/hr being produced by the process

$345.3 \mathrm{~kg}$ of fermentable sugar $/ \mathrm{hr} \cdot 24 \mathrm{hr} / \mathrm{day} \cdot 5$ days $=4,436 \mathrm{~kg}$ sugar produced by the process over the course of 5 days

Corresponding nutrient medium required:

$0.0149 \mathrm{~kg}$ sugar/ $0.04 \mathrm{~L}$ nutrient medium $=4,436 \mathrm{~kg}$ sugar $/ \mathrm{X}$

$\mathrm{X}=11,909 \mathrm{~L}$ nutrient medium (nutrient medium required for 5 days)

Nutrient medium required on a per hour basis:

$11,909 \mathrm{~L} / 5$ days/24hours/day $=100 \mathrm{~L} / \mathrm{hr}$ (water) with $1 \mathrm{~kg}$ of glucose $/ \mathrm{hr}$ (see Table A.15) 
Table A.15: Contents of nutrient medium (Syed, 2012)

\begin{tabular}{|l|l|l|l|l|}
\hline Material & $\begin{array}{l}\text { Amount }(\mathrm{kg})(\text { in } 0.2 \mathrm{~L} \\
\text { water) }\end{array}$ & $\begin{array}{l}\text { Amount in 1L of water } \\
(\mathrm{kg} / \mathrm{L})\end{array}$ & $\begin{array}{l}\text { Amount in 11,909 L } \\
\text { required for 5 days (kg) }\end{array}$ & $\begin{array}{l}\text { Amount in 100L of water } \\
(\mathrm{amount} \text { required/hr) }\end{array}$ \\
\hline Glucose & 0.002 & 0.01 & 119.09 & $1 \mathrm{~kg}$ \\
\hline MgSO4 & 0.00006 & 0.0003 & 3.57 & $0.03 \mathrm{~kg}$ \\
\hline FeSO4 & 0.000002 & 0.00001 & 0.12 & Considered insignificant \\
\hline PAPA & 0.0000002 & 0.000001 & 0.01 & Considered insignificant \\
\hline Biotin & 0.000004 & 0.00002 & 0.24 & Considered insignificant \\
\hline Thiamin & 0.00000002 & 0.0000001 & 0.001 & Considered insignificant \\
\hline $\begin{array}{l}\text { Casein } \\
\text { hydrolysate }\end{array}$ & 0.0008 & 0.004 & 47.636 & $0.4 \mathrm{~kg}$ \\
\hline
\end{tabular}

GaBi has a flow steam of glucose syrup $(68 \mathrm{w} \%)$ that would be substituted for the nutrient source (if available)

$1 \mathrm{~kg}$ of glucose is transported with $1.4 \mathrm{~kg}$ of water (for GaBi glucose syrup stream)

$1 \mathrm{~kg}$ sugar/ $1.4 \mathrm{~kg}$ water $=0.68$

Total mass of solution is $2.4 \mathrm{~kg}$

To use the glucose, the solution needs to be diluted to $1 \mathrm{~kg} / 100 \mathrm{~L}(100 \mathrm{~L}$ of water $=100 \mathrm{~kg})$

Additional water required is $100 \mathrm{~kg}-1.4 \mathrm{~kg}=98.6 \mathrm{~kg}$ water

Output \#17, 18, 19 - bio-acetone, bio-butanol and bio-ethanol

Fermented sugar $=421.10 \mathrm{~kg} / \mathrm{hr} \cdot 82 \%=345.3 \mathrm{~kg} / \mathrm{hr}$

$345.3 \mathrm{~kg}$ fermented sugar $/ \mathrm{hr} \cdot 49 \%=169.2 \mathrm{~kg} \mathrm{ABE} / \mathrm{hr}$

Bio-acetone produced: 
$169.2 \mathrm{~kg} \mathrm{ABE} / \mathrm{hr} \cdot 0.3=50.76 \mathrm{~kg}$ bio-acetone $/ \mathrm{hr}$

Bio-butanol produced:

$169.2 \mathrm{~kg} \mathrm{ABE} / \mathrm{hr} \cdot 0.6=101.52 \mathrm{~kg}$ bio-butanol $/ \mathrm{hr}$

Bio-ethanol produced:

$169.2 \mathrm{~kg} \mathrm{ABE} / \mathrm{hr} \cdot 0.1=16.92 \mathrm{~kg}$ bio-ethanol $/ \mathrm{hr}$

\section{Ouput \#22 - Un-reacted (UR) lignocellulosic biomass}

$421.10 \mathrm{~kg}$ fermentable lignocellulosic biomass $/ \mathrm{hr}-345.3 \mathrm{~kg}$ fermented lignocellulosic biomass $/ \mathrm{hr}$

Un-reacted sugar $=75.8 \mathrm{~kg} / \mathrm{hr}$

Output \#23 - carbon dioxide produced

Details/Assumptions:

- $\quad$ Basic reaction: Total sugars $\rightarrow \mathrm{ABE}+$ acetic acid + butyric acid + carbon dioxide + hydrogen

- By-products include acetic acid (5\%), butyric acid (2\%) (Begum \& Dahman, 2015)

- $\quad 93 \%$ of products of the above reaction is carbon dioxide and hydrogen. Of this $93 \%, 95 \%$ is $\mathrm{CO}_{2}$ and $5 \%$ is $\mathrm{H}_{2}$ (Kótai et al., 2013)

Calculations:

$345.3 \mathrm{~kg}$ fermented sugar $/ \mathrm{hr} \cdot 51 \%=176.10 \mathrm{~kg}$ by-products $/ \mathrm{hr}$

Amount of $\mathrm{CO}_{2}$ produced:

$176.10 \mathrm{~kg}$ by-products $/ \mathrm{hr}(0.93 \cdot 0.95)=155.58 \mathrm{~kg} \mathrm{CO}_{2} / \mathrm{hr}=1.26 \times 10^{\wedge} 6 \mathrm{~kg} \mathrm{CO}_{2} /$ year (assuming 47 week year) 


\section{A.5.6 EVP 05 \& 06 - Evaporate bio-acetone and bio-ethanol (input \#28, 29, 30, 31)}

\section{Details/Assumptions}

- Distillation is a physical separation method used to separate compounds from a mix based on the boiling points of the compounds in the mixture. Vaporization here occurs from the whole liquid mass and not just the surface when the mixture's temperature is increased by an outside source up to the boiling point of the compound of interest (Vobis LLC, 2017)

- The reboiler is the means of heating the column and re-circulating the feed stream for continued evaporation

- Acetone's boiling point is $56^{\circ} \mathrm{C}$ (Green \& Perry, 2007), therefore for EVP $06 \mathrm{~T}_{\text {in }}=45^{\circ} \mathrm{C}, \mathrm{T}_{\text {out }}=56^{\circ} \mathrm{C}$

- Ethanol's boiling point is $79^{\circ} \mathrm{C}\left(\right.$ Green \& Perry, 2007), therefore for EVP $07 \mathrm{~T}_{\text {out }}=79^{\circ} \mathrm{C}$

- Total evaporator column heating requirements are from 45 to $79^{\circ} \mathrm{C}$

- Additional water (for pervaporation) is required to separate the azeotropes produced between existing water and the alcohol in the feed stream such that there is only need for 1 distillation column (Martin, 1998). Water feed flow rate: $174 \mathrm{~kg} / \mathrm{hr}$ used in a similar scale process (Giang et al., 2017) (pg 68 of reference)

- Assume chitosan membrane material does not have a significant environmental impact considering the longevity of the membrane and its disposability if manufactured without the use of silica instead of poly(ethylene) glycol (Clasen et al. 2006)

- Using NG for heat source and water for refrigerant medium 
Table A.16: Properties of ABE (Basf Petronas Chemicals, $2006(\#)$; Wright, 2011), Cp = A + BT + CT^2 (J/gmol·K)

\begin{tabular}{|l|l|l|l|l|l|l|}
\hline \multicolumn{3}{|c|}{} & \multicolumn{2}{l}{$\begin{array}{l}\text { Heat capacity coefficients at constant pressure } \\
\text { (gas/liquid) }\end{array}$} & \\
\hline Compound & $\begin{array}{l}\text { Molecular } \\
\text { weight } \\
(\mathrm{g} / \mathrm{mol})\end{array}$ & $\begin{array}{l}\text { Heat of } \\
\text { vaporization }\end{array}$ & A & B & C & $\begin{array}{l}\text { Liquid heat capacity } \\
(\mathrm{Cp})\end{array}$ \\
\hline Acetone & $58 \mathrm{~g} / \mathrm{mol}$ & $29.1 \mathrm{~kJ} / \mathrm{gmol}$ & $6.301 / 72.2$ & $0.261 / 0.186$ & $-1.25 \times 10^{\wedge}-4$ & \\
\hline Ethanol & $46 \mathrm{~g} / \mathrm{mol}$ & $38.6 \mathrm{~kJ} / \mathrm{gmol}$ & 9.014 & 0.214 & $-8.39 \times 10^{\wedge}-4$ & $112.0 \mathrm{~J} / \mathrm{gmol} \cdot \mathrm{K}$ \\
\hline Butanol & $74 \mathrm{~g} / \mathrm{mol}$ & $592 \mathrm{~kJ} / \mathrm{kg}(\#)$ & & & & $2.589 \mathrm{~kJ} / \mathrm{kg} \cdot \mathrm{K}(\#)$ \\
\hline
\end{tabular}

Calculations:

Input \#28 - water for pervaporation

Water feed flow rate: $174 \mathrm{~kg} / \mathrm{hr}$ (see Detals/Assumptions)

Input \#29 - pervaporation water flow energy

Flow rate in $\mathrm{m}^{3}: 0.174 \mathrm{~m}^{3} / \mathrm{hr}$ required (assuming density of water $1000 \mathrm{~kg} / \mathrm{m}^{3}$ )

Pelect $(\mathrm{kW})=\mathrm{Q}\left(\mathrm{m}^{3} / \mathrm{hr}\right) \cdot \Delta \mathrm{P}($ bar $) / 36 \cdot \mathrm{E}(\% / 100)$

$\mathrm{E}=$ efficiency of (pump, transmission, motor)

Pelect $=0.174 \mathrm{~m}^{3} / \mathrm{hr} \cdot 1 \mathrm{bar} / 36 \cdot 0.75$

Pelect $=6.4 \times 10-3 \mathrm{~kW}$ (power)

Input \#30 - pervaporation chitosan membrane

- 141 day lifespan = 2.3 membranes $/ 47$ week year

- $35 \mathrm{~m}^{2}$ of membrane material used in a similar scale process (Giang et al., 2017) (pg 70 of reference) 
Input \#31 -heat energy

Table A.17: Properties of $\mathrm{ABE}$ in distillation column for increase in temperature from $45^{\circ} \mathrm{C}$ to $56^{\circ} \mathrm{C}$

\begin{tabular}{|l|l|l|l|l|l|}
\hline Compound & $\begin{array}{l}\text { Molar mass } \\
(\mathrm{g} / \mathrm{mol})\end{array}$ & $\begin{array}{l}\text { Mass flow rate } \\
(\mathrm{kg} / \mathrm{hr})\end{array}$ & $\begin{array}{l}\text { Molar flow rate } \\
(\mathrm{mol} / \mathrm{hr})(\mathrm{ni})\end{array}$ & Enthalpy designate & Enthalpy $(\mathrm{kJ} / \mathrm{mol})$ \\
\hline Acetone & 58.08 & 50.76 & 874 & $\mathrm{H} 1$ & 30.0 \\
\hline Butanol & 74.12 & 101.52 & 1370 & $\mathrm{H} 2$ & 2.1 \\
\hline Ethanol & 46.07 & 16.92 & 367 & $\mathrm{H} 3$ & 1.2 \\
\hline
\end{tabular}

Heat required to increase $\mathrm{ABE}$ steam temperature from $45^{\circ} \mathrm{C}$ to $56^{\circ} \mathrm{C}$ :

$\mathrm{H} 1=\int(329 \mathrm{~K}-318 \mathrm{~K}) \mathrm{Cp}(1)(\mathbf{A C E}) \mathrm{dT}+\Delta \mathrm{H}$ vap (evaporating acetone)

$\mathrm{H} 1=6.301 \mathrm{~T}+\left(0.261 \mathrm{~T}^{\wedge} 2\right) / 2-\left(1.25 \times 10^{\wedge}-4 \mathrm{~T}^{\wedge} 3\right) / 3(\mathrm{~J} / \mathrm{gmol})+\Delta \mathrm{H}$ vap

$\mathrm{H} 1=6.301(329-318)+\left(0.261\left(329^{\wedge} 2-318^{\wedge} 2\right)\right) / 2-\left(1.25 \times 10^{\wedge}-4\left(329^{\wedge} 3-318^{\wedge} 3\right)\right) / 3+29.1 \mathrm{~kJ} / \mathrm{gmol}$

$\mathrm{H} 1=854.2 \mathrm{~J} / \mathrm{gmol}+29.1 \mathrm{~kJ} / \mathrm{gmol}$

$\mathrm{H} 1=0.854 \mathrm{~kJ} / \mathrm{gmol}+29.1 \mathrm{~kJ} / \mathrm{gmol}$

$\mathrm{H} 1=30.0 \mathrm{~kJ} / \mathrm{mol}$

$\mathrm{H} 2=\int(329 \mathrm{~K}-318 \mathrm{~K}) \mathrm{Cp}(\mathrm{l})(\mathbf{B U T}) \mathrm{dT}$

$\mathrm{H} 2=(329-318) \mathrm{Cp}(1)$

$\mathrm{H} 2=(329-318) \cdot 2.589 \mathrm{~kJ} / \mathrm{kg} \cdot \mathrm{K} \cdot 0.07412 \mathrm{~kg} / \mathrm{mol}$

$\mathrm{H} 2=(329-318) \cdot 0.192 \mathrm{~kJ} / \mathrm{mol} \cdot \mathrm{K}$

$\mathrm{H} 2=2.1 \mathrm{~kJ} / \mathrm{mol}$ 


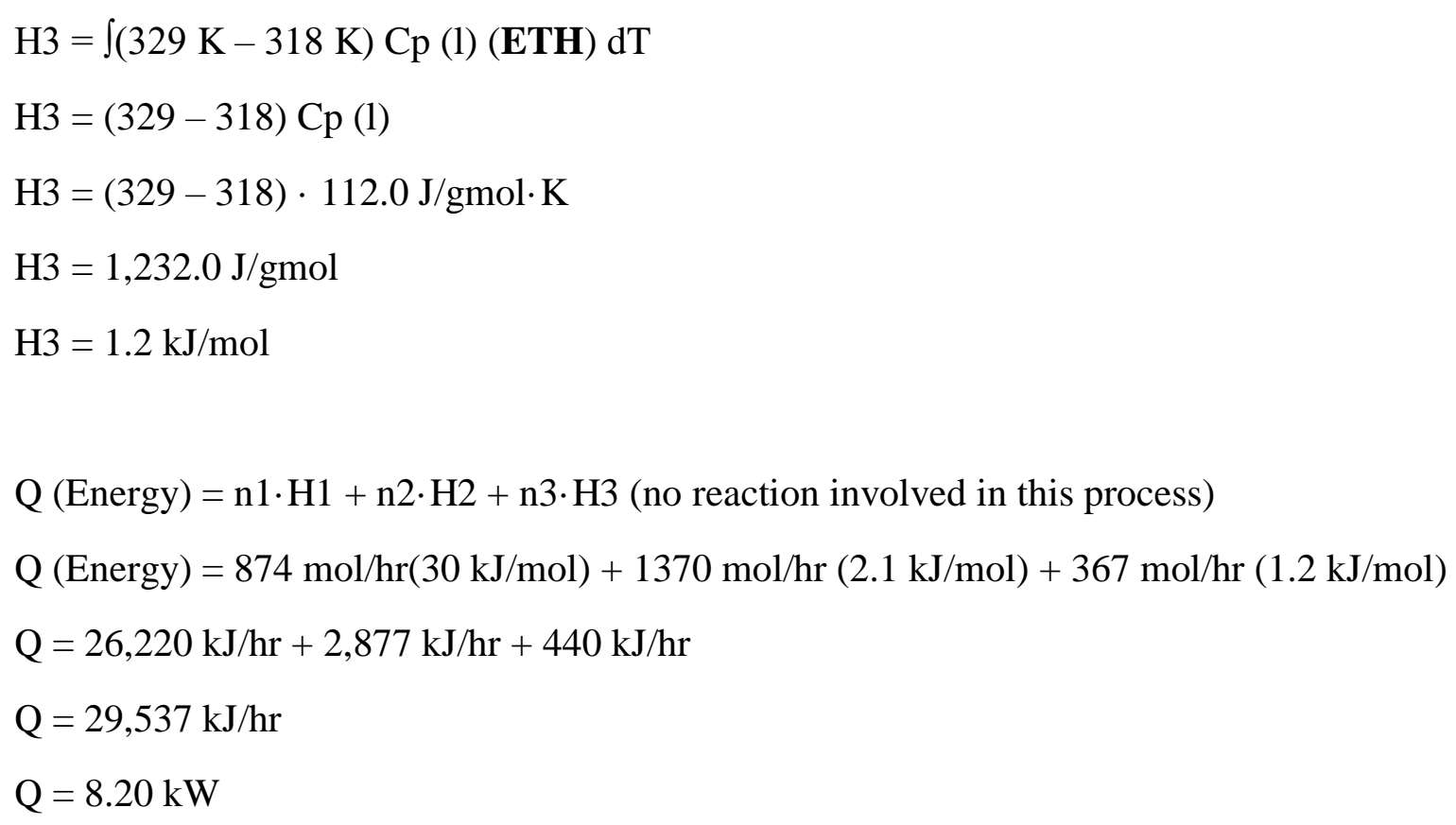

Heat required to increase $\mathrm{BE}$ stream temperature from $56^{\circ} \mathrm{C}$ to $79^{\circ} \mathrm{C}$

Table A.18: Properties of ABE in distillation column for increase in temperature from $56^{\circ} \mathrm{C}$ to $79^{\circ} \mathrm{C}$

\begin{tabular}{|l|l|l|l|l|l|}
\hline Compound & $\begin{array}{l}\text { Molar mass } \\
(\mathrm{g} / \mathrm{mol})\end{array}$ & $\begin{array}{l}\text { Mass flow rate } \\
(\mathrm{kg} / \mathrm{hr})\end{array}$ & $\begin{array}{l}\text { Molar flow rate } \\
(\mathrm{mol} / \mathrm{hr})(\mathrm{ni})\end{array}$ & Enthalpy designate & Enthalpy $(\mathrm{kJ} / \mathrm{mol})$ \\
\hline Butanol & 74.12 & 101.52 & 1370 & $\mathrm{H} 1$ & 4.4 \\
\hline Ethanol & 46.07 & 16.92 & 367 & $\mathrm{H} 2$ & 39.9 \\
\hline
\end{tabular}

$\mathrm{H} 1=\int(352 \mathrm{~K}-329 \mathrm{~K}) \mathrm{Cp}(\mathrm{l})(\mathbf{B U T}) \mathrm{dT}$

$\mathrm{H} 1=(352-329) \mathrm{Cp}(\mathrm{l})$ 
$\mathrm{H} 1=(352-329) \cdot 2.589 \mathrm{~kJ} / \mathrm{kg} \cdot \mathrm{K} \cdot 0.07412 \mathrm{~kg} / \mathrm{mol}$

$\mathrm{H} 1=(352-329) \cdot 0.192 \mathrm{~kJ} / \mathrm{mol} \cdot \mathrm{K}$

$\mathrm{H} 1=4.4 \mathrm{~kJ} / \mathrm{mol}$

$\mathrm{H} 2=\int(352 \mathrm{~K}-329 \mathrm{~K}) \mathrm{Cp}(\mathrm{l})(\mathbf{E T H}) \mathrm{dT}+\Delta \mathrm{H}$ vap (evaporating (bio)ethanol)

$\mathrm{H} 2=9.014 \mathrm{~T}+\left(0.214 \mathrm{~T}^{\wedge} 2\right) / 2-\left(8.39 \times 10^{\wedge}-4 \mathrm{~T}^{\wedge} 3\right) / 3(\mathrm{~J} / \mathrm{gmol})+\Delta \mathrm{H}$ vap

$\mathrm{H} 2=9.014(352-329)+\left(0.214\left(352^{\wedge} 2-329^{\wedge} 2\right)\right) / 2-\left(8.39 \times 10^{\wedge}-4\left(352^{\wedge} 3-329^{\wedge} 3\right)\right) / 3+38.6 \mathrm{~kJ} / \mathrm{gmol}$

$\mathrm{H} 2=1,344.89 \mathrm{~J} / \mathrm{gmol}+38.6 \mathrm{~kJ} / \mathrm{gmol}$

$\mathrm{H} 2=1.34 \mathrm{~kJ} / \mathrm{gmol}+38.6 \mathrm{~kJ} / \mathrm{gmol}$

$\mathrm{H} 2=39.9 \mathrm{~kJ} / \mathrm{mol}$

$\mathrm{Q}($ Energy $)=\mathrm{n} 1 \mathrm{H} 1+\mathrm{n} 2 \mathrm{H} 2($ no reaction involved in this process $)$

$\mathrm{Q}($ Energy $)=1,370 \mathrm{~mol} / \mathrm{hr}(4.4 \mathrm{~kJ} / \mathrm{mol})+367 \mathrm{~mol} / \mathrm{hr}(39.9 \mathrm{~kJ} / \mathrm{mol})$

$\mathrm{Q}=6,028 \mathrm{~kJ} / \mathrm{hr}+14,643 \mathrm{~kJ} / \mathrm{hr}$

$\mathrm{Q}=20,671 \mathrm{~kJ} / \mathrm{hr}$

$\mathrm{Q}=5.74 \mathrm{~kW}$

A.5.7 CND 04 - Bio-acetone condenser (input \#33)

Details/Assumptions: 
- $\quad \mathrm{T}_{\text {in }}=56^{\circ} \mathrm{C}, \mathrm{T}_{\text {out }}=25^{\circ} \mathrm{C}$

- Completely condense bio-acetone to a liquid for transport

Calculations:

$\mathrm{Q}=\mathrm{n} 1 \Delta$ Hvap (bio-acetone)

$\mathrm{Q}=50.8 \mathrm{~kg} / \mathrm{hr} / 0.058 \mathrm{~kg} / \mathrm{mol} \cdot 29.1 \mathrm{~kJ} / \mathrm{mol}$

$\mathrm{Q}=25,488 \mathrm{~kJ} / \mathrm{hr}$

$\mathrm{Q}=7.1 \mathrm{~kW}$

Heat capacity of water at $25^{\circ} \mathrm{C}=4.184 \mathrm{~kJ} / \mathrm{kg} \cdot \mathrm{K}$

$\mathrm{Q}=\mathrm{mCp} \Delta \mathrm{T}$

$-7.1 \mathrm{~kJ} / \mathrm{s}=\mathrm{m}(4.18 \mathrm{~kJ} / \mathrm{kg} \cdot \mathrm{K})(56-25) \mathrm{K}$

Mass of water to cool $=7.1 \mathrm{~kJ} / \mathrm{s} / 129.58 \mathrm{~kJ} / \mathrm{kg}=0.054 \mathrm{~kg} / \mathrm{s}$

A.5.8 CND 05 - Bio-ethanol condenser (input \#38)

Details/Assumptions:

- $\quad \mathrm{T}_{\text {in }}=79^{\circ} \mathrm{C}, \mathrm{T}_{\text {out }}=25^{\circ} \mathrm{C}$

- Completely condense bio-ethanol to liquid for transport

Calculations

$\mathrm{Q}=\mathrm{n} 1 \Delta$ Hvap (bio-ethanol)

$\mathrm{Q}=16.9 \mathrm{~kg} / \mathrm{hr} / 0.046 \mathrm{~kg} / \mathrm{mol} \cdot 38.6 \mathrm{~kJ} / \mathrm{mol}$ 
$\mathrm{Q}=14,181 \mathrm{~kJ} / \mathrm{hr}$

$\mathrm{Q}=3.9 \mathrm{~kW}$

A.6 IO for Anaerobic Digester and Co-generation facility

Table A.19: Anaerobic digestion and co-generation facility process input/output table

\begin{tabular}{|c|c|c|c|c|c|c|}
\hline Stage & & Inputs & Input amount & & Outputs & Output amount \\
\hline $\begin{array}{l}\text { AD (AND } \\
01)\end{array}$ & $\begin{array}{l}1 \\
2\end{array}$ & $\begin{array}{l}\text { LO lipids (from BD) } \\
\text { LO lignocellulosic } \\
\text { biomass (from ABE) } \\
\text { Protein } \\
\text { Power }\end{array}$ & $\begin{array}{l}6.85 \mathrm{~kg} / \mathrm{hr} \\
\mathbf{9 8} \mathrm{kg} / \mathrm{hr} \\
380 \mathrm{~kg} / \mathrm{hr} \\
53.3 \mathrm{kWh} \text { (1 hour) }\end{array}$ & $\begin{array}{l}1 \\
2 \\
3\end{array}$ & $\begin{array}{l}\text { Biogas }\left(\mathrm{CO}_{2}\right) \\
\text { Biogas }\left(\mathrm{CH}_{4}\right) \\
\text { Biosolids }(\mathrm{N} \& \mathrm{P})\end{array}$ & $\begin{array}{l}3.1 \times 10^{\wedge} 6 \mathrm{kgCO}_{2} / \text { year } \\
\mathbf{1 . 4 5 x 1 0}^{\wedge} \mathbf{~ k g ~ C H} 4 / \text { year } \\
2.05 \mathrm{~kg} \mathrm{~N} / \mathrm{hr} \\
2.59 \mathrm{~kg} \mathrm{P} / \mathrm{hr}\end{array}$ \\
\hline $\begin{array}{l}\text { Gas stripping } \\
\text { (SRP 01) }\end{array}$ & $\begin{array}{l}5 \\
6 \\
7\end{array}$ & $\begin{array}{l}\text { Biogas }\left(\mathrm{CO}_{2}\right) \\
\text { Biogas }\left(\mathbf{C H}_{4}\right) \\
\text { Power }\end{array}$ & $\begin{array}{l}3.1 \times 10^{\wedge} 6 \mathrm{kgCO}_{2} \\
\text { /year } \\
\mathbf{1 . 4 5 \times 1 0} \mathbf{1 0}^{\wedge} \mathbf{6} \mathbf{~ k g} \\
\mathbf{C H} 4 / \text { year } \\
80.1 \mathrm{kWh} \text { (1 hour) }\end{array}$ & $\begin{array}{l}4 \\
5\end{array}$ & $\begin{array}{l}\text { Biogas }\left(\mathrm{CO}_{2}\right) \\
\text { Biogas }\left(\mathbf{C H}_{4}\right)\end{array}$ & $\begin{array}{l}3.1 \times 10^{\wedge} 6 \mathrm{kgCO}_{2} / \text { year } \\
\mathbf{1 . 4 5 \times 1 0}^{\wedge} \mathbf{6 g ~} \mathbf{~ C H}_{4} / \text { year }\end{array}$ \\
\hline $\begin{array}{l}\text { Power } \\
\text { generation } \\
(\text { COG 01) }\end{array}$ & 8 & Biogas $\left(\mathrm{CH}_{4}\right)$ & $\begin{array}{l}1.45 \times 10^{\wedge} 6 \mathrm{~kg} \\
\mathrm{CH}_{4} / \text { year }\end{array}$ & $\begin{array}{l}6 \\
7\end{array}$ & $\begin{array}{l}\text { Power } \\
\text { Flue gas }\end{array}$ & $\begin{array}{l}\text { 2,603.25 MJ/hr } \\
\text { Recycled to PBR }\end{array}$ \\
\hline
\end{tabular}

A.6.1 AND 01 - Anaerobic digester (input \#4, output \#1, 2, 3)

Details/Assumptions

- Density of $\mathrm{CO}_{2}=1.98 \mathrm{~kg} / \mathrm{m}^{3}$ (Green \& Perry 2007)

- Anaerobic digester biogas content is 50-70\% methane $\left(\mathrm{CH}_{4}\right)$ and 30-50\% $\mathrm{CO}_{2}$ with trace amounts of $\mathrm{H}_{2} \mathrm{~S}$ (Yanwen Shen et al., 2016). This study assumes $\mathrm{CO}_{2}: \mathrm{CH}_{4}$ in biogas is $60: 40$ 
- Density of methane: $0.6987 \mathrm{~kg} / \mathrm{m}^{3}$ at $298 \mathrm{~K} 1 \mathrm{~atm}, 0.777 \mathrm{~kg} / \mathrm{m}^{3}$ at STP (Green \& Perry 2007)

- $\quad$ Methane yield from Table 2.11 in section 2.4.7

- One $\mathrm{kg}$ of nitrogen and $1 \mathrm{~kg}$ of phosphorus from solid digestate (AD) is substituted for $0.6 \mathrm{~kg}$ of nitrogen and $0.4 \mathrm{~kg}$ of phosphorus in synthetic fertilizer respectively (Yuan et al., 2015)

- Electrical power requirements for mixing are $0.11 \mathrm{kWh} / \mathrm{kg}$-TS (Collet et al., 2011)

Calculations:

Input \#4 - power for the additional digestate production

Total solids (TS) added to digester form microalgal process $=6.85 \mathrm{~kg}$ LO lipids $/ \mathrm{hr}+98 \mathrm{~kg}$ LO \& UR lignocellulosic biomass $/ \mathrm{hr}+380$ $\mathrm{kg}$ protein $/ \mathrm{hr}=484.85 \mathrm{~kg}-\mathrm{TS} / \mathrm{hr}$

$484.85 \mathrm{~kg}-\mathrm{TS} / \mathrm{hr} \cdot 0.11 \mathrm{kWh} / \mathrm{kg}-\mathrm{TS}=53.3 \mathrm{kWh}$

\section{$\underline{\text { Output \#1 - carbon dioxide }}$}

Carbon dioxide produced from un-extracted and unreacted lipid portion of microalgal biomass sent to anaerobic digester:

$6.85 \mathrm{~kg}$ lipids not used $/ \mathrm{hr}$

$6.85 \mathrm{~kg}$ lipids to digester $/ \mathrm{hr} \cdot 1 \mathrm{~m}^{3}$ methane $/ \mathrm{kg}$ fat $=6.85 \mathrm{~m}^{3}$ methane $/ \mathrm{hr}$

Using 60:40 ratio, $6.85 \mathrm{~m}^{3}$ methane $/ \mathrm{hr}=10.28 \mathrm{~m}^{3} \mathrm{CO}_{2} / \mathrm{hr}$ produced

$10.28 \mathrm{~m}^{3} \mathrm{CO}_{2} / \mathrm{hr}$ produced $\cdot 1.98 \mathrm{~kg} / \mathrm{m}^{3}=20.34 \mathrm{~kg} \mathrm{CO} / \mathrm{hr}$ or $7.14 \times 10^{\wedge} 5 \mathrm{~kg} \mathrm{CO}_{2} /$ year

Carbon dioxide produced from proteins sent to anaerobic digester:

$380 \mathrm{~kg}$ proteins $/ \mathrm{hr} \cdot 24 \mathrm{hr} / \mathrm{day} \cdot 7$ days/week $\cdot 47$ weeks/year $=3,000,480 \mathrm{~kg}$ protein to digester $/$ year 
$3,000,480 \mathrm{~kg}$ lipids to digester $/$ year $\cdot 0.51 \mathrm{~m}^{3}$ methane $/ \mathrm{kg}$ protein $=1,530,245 \mathrm{~m}^{3}$ methane

Using 60:40 ratio, 1,020,163 $\mathrm{m}^{3} \mathrm{CO}_{2}$ /year produced

$1,020,163 \mathrm{~m}^{3} \mathrm{CO}_{2} /$ year produced $\cdot 1.98 \mathrm{~kg} / \mathrm{m}^{3}=2,019,923 \mathrm{kgCO}_{2} /$ year

Carbon dioxide produced by sending un-extracted and uncreated lignocellulosic biomass to anaerobic digester:

$98 \mathrm{~kg}$ lignocellulosic/hr$\cdot 24 \mathrm{hr} /$ day· 7 days/week 47 weeks/year $=773,808 \mathrm{~kg}$ lignocellulosic to digester $/$ year

$773,808 \mathrm{~kg}$ lignocellulosic to digester $/$ year $\cdot 0.37 \mathrm{~m}^{3}$ methane $/ \mathrm{kg}$ lignocellulose $=286,309 \mathrm{~m}^{3}$ methane

Using 60:40 ratio, $190,873 \mathrm{~m}^{3} \mathrm{CO}_{2}$ /year produced

$190,873 \mathrm{~m}^{3} \mathrm{CO}_{2}$ /year produced $\cdot 1.98 \mathrm{~kg} / \mathrm{m}^{3}=377,928 \mathrm{~kg} \mathrm{CO}_{2} /$ year

Total $\mathrm{CO}_{2}$ produced from $\mathrm{AD}$ due to microalgal biomass waste:

$7.14 \times 10^{\wedge} 5 \mathrm{~kg} \mathrm{CO}_{2} /$ year (from lipids) $+2,019,923 \mathrm{kgCO}_{2} /$ year (from protein) $+377,928 \mathrm{~kg} \mathrm{CO} /$ year (from lignocellulosic) $=$ $3.1 \times 10^{\wedge} 6 \mathrm{kgCO}_{2} /$ year

\section{Output \#2 - methane}

Methane produced by sending left over lipids to anaerobic digester:

$6.85 \mathrm{~kg}$ lipids not used/hr $\cdot 24 \mathrm{hr} / \mathrm{day} \cdot 7$ days $/$ week $\cdot 47$ weeks $/$ year $=54,087.6 \mathrm{~kg}$ lipids to digester $/$ year $54,088 \mathrm{~kg}$ lipids to digester $/$ year $\cdot 1 \mathrm{~m}^{3}$ methane $/ \mathrm{kg}$ fat $=54,088 \mathrm{~m}^{3}$ methane

$54,088 \mathrm{~m}^{3}$ methane $\cdot 0.777 \mathrm{~kg}$ methane $/ \mathrm{m}^{3}=42,026.4 \mathrm{~kg} \mathrm{CH} /$ year

Methane produced by sending proteins to anaerobic digester: 
$1,530,245 \mathrm{~m}^{3}$ methane $\cdot 0.777 \mathrm{~kg}$ methane $/ \mathrm{m}^{3}=1,189,000.4 \mathrm{~kg} \mathrm{CH} /$ year

Methane produced by sending LO and UR lignocellulosic biomass to anaerobic digester:

$286,309 \mathrm{~m}^{3}$ methane $\cdot 0.777 \mathrm{~kg}$ methane $/ \mathrm{m}^{3}=222,461 \mathrm{~kg} \mathrm{CH} /$ year

Total $\mathrm{CH}_{4}$ produced from $\mathrm{AD}$ due to microalgal biomass waste:

42,026.4 kg CH $4 /$ year from lipids + 1,189,000.4 kg CH $4 /$ year from protein $+222,461 \mathrm{~kg} \mathrm{CH} /$ year from LO and UR lignocellulosic biomass $=1,453,488 \mathrm{~kg} \mathrm{CH}_{4} /$ year

\section{$\underline{\text { Output \#3 - Biosolids }}$}

Total amount of $\mathrm{N}$ and $\mathrm{P}$ in wastewater using Ma (2016) values that will be recycled every hour from the AD:

$0.178 \mathrm{~g} \mathrm{P} / \mathrm{L} \cdot 1.15 \times 10^{\wedge} 8 \mathrm{~L} /$ year $=2.05 \times 10^{\wedge} 7 \mathrm{~g} /$ year $=2.59 \mathbf{~ k g ~ P} / \mathbf{h r}$

$0.141 \mathrm{~g} \mathrm{~N} / \mathrm{L} \cdot 1.15 \times 10^{\wedge} 8 \mathrm{~L} /$ year $=1.62 \times 10^{\wedge} 7 \mathrm{~g} /$ year $=\mathbf{2 . 0 5} \mathbf{~ k g ~ N} / \mathbf{h r}$

$1 \mathrm{~kg} \mathrm{~N}$ from $\mathrm{AD} / 0.6 \mathrm{~kg}$ of $\mathrm{N}$ in synthetic fertilizer $=2.05 \mathrm{~kg} \mathrm{~N}$ from $\mathrm{AD} / \mathrm{X}$

$\mathrm{X}=\mathrm{kg} \mathrm{N}$ in synthetic fertilizer $=1.23 \mathrm{~kg} \mathrm{~N} / \mathrm{hr}$

$1 \mathrm{~kg}$ of $\mathrm{P}$ from $\mathrm{AD} / 0.4 \mathrm{~kg}$ of $\mathrm{P}$ in synthetic fertilizer $=2.59 \mathrm{~kg} \mathrm{P}$ from $\mathrm{AD} / \mathrm{X}$

$\mathrm{X}=\mathrm{kg} \mathrm{P}$ in synthetic fertilizer $=1.04 \mathrm{~kg} \mathrm{P} / \mathrm{hr}$ 
A.6.2 SRP 01 - Stripping biogas and fermenter gas (input 7)

Details/Assumptions:

- $\quad$ stripping energy requirements range from 0.15 to $0.5 \mathrm{kWh} / \mathrm{Nm}^{3}$ ( $\mathrm{N}$ refers to the temperature measurement at STP) (Bauer et al., 2013).

Calculations:

[54,088 $\mathrm{m}^{3} \mathrm{CH}_{4}$ from LO lipids/year $+1,530,245 \mathrm{~m}^{3} \mathrm{CH}_{4}$ from protein/year $+286,309 \mathrm{~m}^{3} \mathrm{CH}_{4}$ from LO and UR lignocellulosic biomass/year $+10.28 \mathrm{~m}^{3} \mathrm{CO}_{2}$ from LO lipids/hr $+1,020,163 \mathrm{~m}^{3} \mathrm{CO}_{2}$ from protein/year $+190,873 \mathrm{~m}^{3} \mathrm{CO}_{2}$ from $\mathrm{LO}_{\text {and }} \mathrm{UR}$ lignocellulosic biomass/year] $/ 47$ weeks/year/7days/week/24 hr/day $=400.56 \mathrm{~m}^{3} \mathrm{gas} / \mathrm{hr}$

$0.2 \mathrm{kWh} / \mathrm{m}^{3} \cdot 400.56 \mathrm{~m}^{3}$ gas $/ \mathrm{hr}=80.1 \mathrm{kWh}(1$ hour $)$

\section{A.6.3 COG 01 - Co-gereration facility for energy production (output \#6):}

Details/Assumptions

- Heat of combustion of methane $=$ lower heating value (LHV) of methane $=47 \mathrm{MJ} / \mathrm{kg}($ Frank et al. 2011)

- $\quad$ power generation systems of less than $5 \mathrm{MW}$ had a power generation conversion efficiency of $30 \%$ (Frank et al. 2011)

Calculations:

1,453,488 kg CH $4 /$ year $\cdot 47.14 \mathrm{MJ} / \mathrm{kg}=68,517,430 \mathrm{MJ} /$ year $=8,677.5 \mathrm{MJ} / \mathrm{hr}$

$8,677.5 \mathrm{MJ} / \mathrm{hr} \cdot 0.3=2,603.25 \mathrm{MJ} / \mathrm{hr}=723 \mathrm{kWh}$

Allocate energy produced between process stages: 
- The percent contribution each stage makes to the overall electrical energy requirements will be the same percentage of the total electrical energy production that is allocated to each process.

- These values will be subtracted from the total electrical power requirements of each stage.

PBR: 4,914 kWh/ 10,874 kWh = 45\%

$0.45 \cdot 723 \mathrm{kWh}=325 \mathrm{kWh}$

Harvest: $5,525 \mathrm{kWh} / 10,874 \mathrm{kWh}=51 \%$

$0.51 \cdot 723 \mathrm{kWh}=369 \mathrm{kWh}$

Separation: $274 \mathrm{kWh} / 10,874 \mathrm{kWh}=3 \%$

$0.03 \cdot 723 \mathrm{kWh}=22 \mathrm{kWh}$

BD production: $9 \mathrm{kWh} / 10,874 \mathrm{kWh}=0 \%$

ABE production: $19.1 \mathrm{kWh} / 10,874 \mathrm{kWh}=0 \%$

AD and power: $133 \mathrm{kWh} / 10,874 \mathrm{kWh}=1 \%$

$0.01 \cdot 723 \mathrm{kWh}=7 \mathrm{kWh}$ 
Appendix B - Pump energy and refrigerant requirements

B.1 Pump energy calculations

Details/Assumptions:

- A 0.5 HP required to circulate water in PBR (Min et al., 2014). The same rating is used for all the pumps in the system moving the main feed as well as providing additional materials (e.g. sodium hydroxide, hexane etc.) as main feed movement is slow

- $\quad$ Energy associated with moving cooling/heating fluids calculated with this equation: $\mathrm{P}_{\text {elect }}(\mathrm{kW})=\mathrm{Q}\left(\mathrm{m}^{3} / \mathrm{hr}\right) \cdot \Delta \mathrm{P}(\mathrm{bar}) / 36 \cdot \mathrm{E}$ (\%/100), E = efficiency of pump (0.75) (Vogelesang, 2008)

- Estimate 7,500 L/PBR based on size of PBR in (Min et al., 2014). Number of PBRs required to process $1.15 \times 10^{\wedge} 8 \mathrm{~L} / \mathrm{day}=$ $1.15 \times 10^{\wedge} 8 \mathrm{~L} /$ day / 7,500 L/PBR $=15,333 \mathrm{PBRs}$

- Estimate 40,000 L/PBR based on size of PBR in Zhou et al. (2014). Number of PBRs required to process $1.15 \times 10^{\wedge} 8 \mathrm{~L} / \mathrm{day}=$ $1.15 \times 10^{\wedge} 8 \mathrm{~L} /$ day / 40,000 L/PBR $=2,875 \mathrm{PBRs}$

Calculations:

$0.5 \mathrm{HP}=0.37285 \mathrm{~kJ} / \mathrm{s} \cdot 3600 \mathrm{~s}=1,342.26 \mathrm{~kJ}=1.3 \mathrm{MJ}$ per pump per hour

Table B.1: List of pumps required - based on diagram in Figure 3.4

\begin{tabular}{|l|l|l|l|}
\hline Stage \# & $\begin{array}{l}\text { Number of } \\
\text { pumps }\end{array}$ & $\begin{array}{l}\text { Process Flow Diagram Equipment } \\
\text { Number }\end{array}$ & Description \\
\hline 1 & n/a & SST01 to PBR01 (2,875) & Wastewater gravity fed \\
\hline 1 & 2,875 & PBR01 (2,875) to FLC01 & Several PBRs supplying water to FLC01 \\
\hline 1 & 1 & TNK05 through 03 to PBR01 & Carbon dioxide to PBR from biogas \\
\hline 1 & 1 & TNK02 to PBR01 & Flue from Co-gen \\
\hline 1 & 1 & AND01 to PBR01 & Supernatant to PBR from AD \\
\hline
\end{tabular}




\begin{tabular}{|c|c|c|c|}
\hline 2 & 1 & FLC01 to CEN01 & Floc to centrifuge \\
\hline 2 & 1 & CEN01 to DRY01 & Centrifuge to dryer \\
\hline 2 & 1 & DRY01 to PUV01 & Dryer to pulverizer \\
\hline 2 & 1 & TNK08 to MXT03 & Sulfuric acid to neutralizing tank for floc water \\
\hline 2 & 1 & MXT03 to SST01 & Returning harvesting water to secondary clarifier \\
\hline 2 & 1 & FLC01 to MXT03 & Floc water to neutralization tank \\
\hline 2 & 1 & CEN01 to MXT03 & Centrifuge water to neutralization tank \\
\hline 2 & 1 & DRY01 to MXT03 & Dryer water to neutralization tank \\
\hline 2 & 1 & TNK06 to FLC01 & Supplying sodium hydroxide \\
\hline 3 & 1 & PUV01 to CSTR01 & Pulverizer to hexane mixing tank \\
\hline 3 & 1 & CSTR01 to CEN02 & Hexane mixing tank to centrifuge for separation \\
\hline 3 & $\mathrm{n} / \mathrm{a}$ & EVP01 to CND01 & Evaporation of hexane to condenser for recirculation \\
\hline 3 & 1 & CND01 to TNK07 & Cooling hexane and recirculation \\
\hline 3 & 1 & TNK07 to CSTR01 & Redistribution of hexane \\
\hline 3 & 1 & EVP01 to EVP07 & Evaporation of ethanol solvent \\
\hline 3 & $\mathrm{n} / \mathrm{a}$ & EVP07 to CND07 & Condensing ethanol solvent \\
\hline 3 & 1 & CND07 to TNK16 & Ethanol solvent circulation to tank \\
\hline 3 & 1 & TNK16 to CSTR01 & Ethanol solvent supplying CSTR with solvent for separation \\
\hline 4 & $\mathrm{n} / \mathrm{a}$ & CEN02 to EVP01 & Oil movement from centrifuge to evaporator \\
\hline 4 & 1 & EVP01 to CMP01 (+1 way vv) & Oil movement from hexane evaporator to compressor \\
\hline 4 & $\mathrm{n} / \mathrm{a}$ & CMP01 to CSTR02 & Compressor to transesterification reactor \\
\hline 4 & 1 & CSTR02 to CLR01 & Cooling products of transesterification reaction \\
\hline 4 & 1 & CLR01 to EVP02 & Evaporating excess methanol \\
\hline 4 & 1 & EVP02 to CND03 & Methanol cooled \\
\hline 4 & 1 & CND03 to TNK09 & Storage tank for methanol \\
\hline 4 & 1 & TNK09 to CMP01 & Recirculation of methanol \\
\hline 4 & 1 & EVP02 to CEN03 & FAME + oil + glycerol to centrifuge for separation \\
\hline 4 & $\mathrm{n} / \mathrm{a}$ & CEN03 to EVP03 & Glycerol moving to evaporator to remove rest of methanol \\
\hline 4 & $\mathrm{n} / \mathrm{a}$ & EVP03 to CND03 & Condensing methanol \\
\hline 4 & 1 & EVP03 to TNK15 & Glycerol intermediate tank \\
\hline 4 & 1 & TNK15 to PBR01 & Glycerol circulated to PBR \\
\hline 4 & $\mathrm{n} / \mathrm{a}$ & CEN03 to EVP04 & FAME + oil to evaporate FAME \\
\hline
\end{tabular}




\begin{tabular}{|l|l|l|l|}
\hline 4 & 1 & EVP04 to TNK14 & Residual oil to intermediate tank \\
\hline 4 & n/a & EVP04 to CND03 & Cooling of evaporated FAME \\
\hline 4 & 1 & CND03 to TNK13 & FAME to storage tank \\
\hline 4 & 1 & TNK14 to AND01 & Residual oil to AD \\
\hline 5 & n/a & CEN02 to MXT01 & Lignocellulosic biomass to pre-treatment tank \\
\hline 5 & 1 & MXT01 to STX01 & Lignocellulosic biomass to steam treat \\
\hline 5 & 1 & STX01 to CLR02 & Cooling after steam treat \\
\hline 5 & 1 & CLR02 to MXT02 & Neutralization after pre treatment \\
\hline 5 & 1 & MXT02 to FRM01 & Pre-treated lignocellulosic biomass to fermenter \\
\hline 5 & 1 & FRM01 to EVP05 & ABE products to st $^{\text {stage evaporator }}$ \\
\hline 5 & 1 & EVP05 to EVP06 & BC to 2 ${ }^{\text {nd } \text { stage evaporator }}$ \\
\hline 5 & 1 & EVP06 to TNK12 & Bio-butanol to storage tank \\
\hline 5 & n/a & EVP05 to CND04 & Cooling bio-acetone \\
\hline 5 & 1 & CND04 to TNK10 & Bio-acetone to storage \\
\hline 5 & n/a & EVP06 to CND05 & Cooling bio-ethanol \\
\hline 5 & 1 & CND05 to TNK11 & Bio-ethanol to storage \\
\hline 5 & 1 & FRM01 to AND01 & Residual lignocellulosic biomass + protein to AD \\
\hline 5 & 1 & FRM01 to TNK05 & Gas purge to stripping system \\
\hline 5 & 1 & TNK08 to MXT01 & Sulfuric acid to pre-treatment tank \\
\hline 5 & 1 & TNK06 to MXT02 & Supplying sodium hydroxide \\
\hline
\end{tabular}

\section{$\underline{\text { PBR }}$}

2,875 PBR with 1 pump each $\cdot 1.3 \mathrm{MJ} / \mathrm{pump} \cdot \mathrm{hr}+3 \mathrm{pumps} \cdot 1.3 \mathrm{MJ} / \mathrm{pump} \cdot \mathrm{hr}=3,741.5 \mathrm{MJ} / \mathrm{hr}$

\section{Harvesting}

9 pumps $\cdot 1.3 \mathrm{MJ} / \mathrm{pump} \cdot \mathrm{hr}=11.7 \mathrm{MJ} / \mathrm{hr}$

$\underline{\text { Separation }}$

7 pumps $\cdot 1.3 \mathrm{MJ} / \mathrm{pump} \cdot \mathrm{hr}=9.1 \mathrm{MJ} / \mathrm{hr}$

$\underline{\text { BD production }}$ 
12 pumps $\cdot 1.3 \mathrm{MJ} / \mathrm{pump} \cdot \mathrm{hr}=15.6 \mathrm{MJ} / \mathrm{hr}$

ABE production

13 pumps $\cdot 1.3 \mathrm{MJ} / \mathrm{pump} \cdot \mathrm{hr}=16.9 \mathrm{MJ} / \mathrm{hr}$

Total power requirement:

$3,741.5 \mathrm{MJ} / \mathrm{hr}+10.4 \mathrm{MJ} / \mathrm{hr}+5.2 \mathrm{MJ} / \mathrm{hr}+15.6 \mathrm{MJ} / \mathrm{hr}+15.6 \mathrm{MJ} / \mathrm{hr}=3,788.3 \mathrm{MJ} / \mathrm{hr}$

Table B.2: Allocation of pump energy requirements by process stage (based on an hourly rating)

\begin{tabular}{|c|c|c|}
\hline Stage \# & Stage & Total electrical energy required (MJ) \\
\hline 1 & PBR & $3,741.5 \mathrm{MJ}$ \\
\hline 2 & Harvesting & $11.7 \mathrm{MJ}$ \\
\hline 3 & Separation & $5.2 \mathrm{MJ}$ \\
\hline 4 & BD production & $15.6 \mathrm{MJ}$ \\
\hline 5 & ABE production & $16.9 \mathrm{MJ}$ \\
\hline
\end{tabular}

B.2 Refrigeration energy calculations

Details/Assumptions:

- Heat capacity of water $=4.184 \mathrm{~kJ} / \mathrm{kg} \cdot \mathrm{K}($ Green $\&$ Perry, 2007)

- $\quad$ Density of water $=1,000 \mathrm{~kg} / \mathrm{m}^{3}$ 
- Dowthern A heat capacity ranges from $1.5 \mathrm{~kJ} / \mathrm{kg} \cdot \mathrm{K}$ at $12^{\circ} \mathrm{C}$ to $2.8 \mathrm{~kJ} / \mathrm{kg} \cdot \mathrm{K}$ at $425^{\circ} \mathrm{C}$ - average heat capacity is $2.15 \mathrm{~kg} / \mathrm{kg} \cdot \mathrm{K}$ (The DOW Chemical Company, 1997)

- Density of Dowthern A at $2.15 \mathrm{~kg} / \mathrm{kg} \cdot \mathrm{K}$ (approximately $225^{\circ} \mathrm{C}$ ): $833.5 \mathrm{~kg} / \mathrm{m}^{3}$ (The DOW Chemical Company, 1997)

\section{B.2.1 Water refrigerant}

Heating and cooling done with water

*Assume heating is all that is required as all water pumped through system will cool and can be used for cooling purposes thereafter.

Table B.3: Heat energy and temperature delta required for calculating heating and cooling water capacity and power circulation requirements

\begin{tabular}{|c|c|c|c|c|}
\hline Stage & Heat energy & $\begin{array}{l}\text { Temperature } \\
\text { delta }\end{array}$ & Stage & Cooling energy \\
\hline EVP01 & $(2,949 \mathrm{MJ} / \mathrm{hr}) 819 \mathrm{kWh}$ & $25^{\circ} \mathrm{C}-68^{\circ} \mathrm{C}(43)$ & CND01 & $(2,949 \mathrm{MJ} / \mathrm{hr}) 819 \mathrm{kWh}$ \\
\hline EVP07 & $(818 \mathrm{MJ} / \mathrm{hr}) 227 \mathrm{kWh}$ & $68^{\circ} \mathrm{C}-79^{\circ} \mathrm{C}(11)$ & CND07 & $(818 \mathrm{MJ} / \mathrm{hr}) 227 \mathrm{kWh}$ \\
\hline EVP03 & $(22 \mathrm{MJ} / \mathrm{hr}) 6 \mathrm{kWh}$ & $25^{\circ} \mathrm{C}-65^{\circ} \mathrm{C}(40)$ & $\mathrm{CND} 02$ & $30.8 \mathrm{kWh}$ \\
\hline EVP05 and EVP06 & $14 \mathrm{kWh}$ & $45^{\circ} \mathrm{C}-79^{\circ} \mathrm{C}(34)$ & $\begin{array}{l}\text { CND04 and } \\
\text { CND05 }\end{array}$ & $11 \mathrm{kWh}$ \\
\hline Total & $1,066 \mathrm{kWh}$ & 128 & & $1,088 \mathrm{kWh}$ \\
\hline
\end{tabular}

Amount of water required to heat to heat capacity:

$\mathrm{Q}=\mathrm{mCp} \Delta \mathrm{T}$ 
$1,066 \mathrm{~kJ} / \mathrm{s}=\mathrm{m}(4.184 \mathrm{~kJ} / \mathrm{kg} \cdot \mathrm{K})(128 \mathrm{~K})$

Mass of water $(\mathrm{m})=1.99 \mathrm{~kg} / \mathrm{s}=7,166 \mathrm{~kg} / \mathrm{hr}$

$7,166 \mathrm{~kg}$ water $/ \mathrm{hr} / 1000 \mathrm{~kg} / \mathrm{m}^{3}=7.166 \mathrm{~m}^{3} / \mathrm{hr}$

Power required to move this amount of water:

$\mathrm{P}_{\text {elect }}(\mathrm{kW})=\mathrm{Q}\left(\mathrm{m}^{3} / \mathrm{hr}\right) \cdot \Delta \mathrm{P}($ bar $) / 36 \cdot \mathrm{E}(\% / 100)$

$\mathrm{P}_{\text {elect }}(\mathrm{kW})=7.166 \mathrm{~m}^{3} / \mathrm{hr} \cdot 1 \mathrm{bar} / 36 \cdot 0.75$

$P_{\text {elect }}=0.265 \mathrm{kWh}(1 \mathrm{MJ} / \mathrm{hr})$

\section{B.2.2 Dowthern A refrigerant}

Heating and Cooling that require by Dowthern:

*Assume heating is all that is required, as all Dowthern A is heated, losing heat while heating and then used to cool thereafter.

Table B.4: Heating energy and temperature delta to calculate corresponding amount of Dowthern A and associated circulation power

\begin{tabular}{|l|l|l|l|l|}
\hline Stage & Heat energy & Temperature delta & Stage & Cooling energy \\
\hline CSTR02 & $42.3 \mathrm{kWh}$ & $25^{\circ} \mathrm{C}-400^{\circ} \mathrm{C}(375)$ & $\mathrm{CLR} 01$ & $50 \mathrm{kWh}$ \\
\hline EVP04 & $73 \mathrm{kWh}$ & $50^{\circ} \mathrm{C}-218^{\circ} \mathrm{C}(168)$ & $\mathrm{CND} 03$ & $35 \mathrm{kWh}$ \\
\hline
\end{tabular}




\begin{tabular}{|l|l|l|l|l|}
\hline STX01 & $\begin{array}{l}(4,176 \mathrm{MJ} / \mathrm{hr}) 1160 \\
\mathrm{kWh}\end{array}$ & $25^{\circ} \mathrm{C}-121^{\circ} \mathrm{C}(96)$ & CLR02 & $(3,306 \mathrm{MJ} / \mathrm{hr}) 918 \mathrm{kWh}$ \\
\hline Total & $\mathbf{1 , 2 7 5} \mathbf{~ k W h}$ & $\mathbf{6 3 9}$ & & $\mathbf{1 , 0 0 3 ~ k W h}$ \\
\hline
\end{tabular}

Amount of Dowthern A required to heat to heat capacity:

$\mathrm{Q}=\mathrm{mCp} \Delta \mathrm{T}$

$1,275 \mathrm{~kJ} / \mathrm{s}=\mathrm{m}(2.15 \mathrm{~kJ} / \mathrm{kg} \cdot \mathrm{K})(639 \mathrm{~K})$

Mass of Dowthern A $(\mathrm{m})=0.919 \mathrm{~kg} / \mathrm{s}=3,311 \mathrm{~kg} / \mathrm{hr}$

$3,311 \mathrm{~kg}$ Dowtern A/hr $/ 833.5 \mathrm{~kg} / \mathrm{m}^{3}=3.972 \mathrm{~m}^{3} / \mathrm{hr}$

Power required to move this amount of water:

$\mathrm{P}_{\text {elect }}(\mathrm{kW})=\mathrm{Q}\left(\mathrm{m}^{3} / \mathrm{hr}\right) \cdot \Delta \mathrm{P}($ bar $) / 36 \cdot \mathrm{E}(\% / 100)$

$\mathrm{P}_{\text {elect }}(\mathrm{kW})=3.972 \mathrm{~m}^{3} / \mathrm{hr} \cdot 1 \mathrm{bar} / 36 \cdot 0.75$

$\mathrm{P}_{\text {elect }}=0.147 \mathrm{kWh}(0.5 \mathrm{MJ} / \mathrm{hr})$

Dowthern A will be continually recycled therefore assuming 5\% loss, only 5\% is needed on a regular basis:

$3,311 \mathrm{~kg}$ Dowtern A·0.05 $=165.6 \mathrm{~kg}$ 
Appendix C - Sourcing materials (distance calculations and assumptions)

Details/Assumptions:

- Toronto's land area is $641 \mathrm{~km}^{2}$ with distances spanning $43 \mathrm{~km}$ east-west and $21 \mathrm{~km}$ north-south (City of Toronto, 2017)

- Given a circular radius of $14.3 \mathrm{~km}\left(\pi \mathrm{r}^{2}=641 \mathrm{~km}^{2}\right)$, there will likely be a factory to source materials within double this distance $\sim 28.6 \mathrm{~km}(27 \mathrm{~km})$

- Used a Euro truck 5, 34-40 t gross weight with a $27 \mathrm{t}$ payload in GaBi for transport for all transport materials

Table C.1: Transport methods for process materials

\begin{tabular}{|c|c|c|}
\hline Materials & Transport methods & $\begin{array}{l}\text { Reference for Transport } \\
\text { method }\end{array}$ \\
\hline Sodium Hydroxide & $\begin{array}{l}\text { Truck in solid form - although } \\
\text { recommended in liquid form }\end{array}$ & (CargoHandbook, 2014) \\
\hline Sulfuric Acid & Truck in liquid form & (CargoHandbook, 2014) \\
\hline $\begin{array}{l}\text { Methanol (NG in } \\
\mathrm{GaBi} \text { ) }\end{array}$ & Truck in liquid form & \\
\hline Hexane & Truck in liquid form & (CargoHandbook, 2014) \\
\hline Nitrogen gas & Truck in liquid form & (CargoHandbook, 2014) \\
\hline Natural gas (NG) & $\begin{array}{l}\text { Normally distributed by existing } \\
\text { pipeline - no transport included }\end{array}$ & \\
\hline Carbon dioxide & $\begin{array}{l}\text { Pipeline required - energy for transport } \\
\text { included only }\end{array}$ & \\
\hline Wastewater & $\begin{array}{l}\text { Co-located with biofuel production } \\
\text { facility - pumping wastewater to PBR } \\
\text { and for further process included }\end{array}$ & \\
\hline Surface water & $\begin{array}{l}\text { From existing surface or ground water } \\
\text { source - pumping energy included }\end{array}$ & \\
\hline $\begin{array}{l}\text { Dowthern A (ethylene } \\
\text { glycol in } \mathrm{GaBi} \text { ) }\end{array}$ & Truck in liquid form & (CargoHandbook, 2014) \\
\hline
\end{tabular}

\section{C.1 Sodium hydroxide}

Details/Assumptions

- $\mathrm{NaOH}$ also called lye

- Stored or transported in pellets or in a $50 \%$ or $70 \%$ saturated solution (called caustic soda liquor) (CargoHandbook, 2014) 
- In GaBi chose $100 \%$ caustic soda instead of liquor as would have had to change water requirements in proceeding processes

\section{C.2 Sulfuric acid}

Details/Assumptions:

- $\quad$ Transported in several concentrations - most common is $93.2 \%$ but $78 \%$ is also popular (CargoHandbook, 2014)

- Acids of $77 \%$ concentration and above do not react with dry mild steel or stainless steel at normal temperatures but dilute acids of less than $77 \%$ concentration will corrode most metals

- Choosing to transport at high concentration and dilute further upon arrival at biorefinery and assuming additional water is negligible.

- $\quad$ Truck in liquid form

\section{C.3 Downthern A}

Details/Assumptions:

- Used ethylene glycol instead of Dowthern A in GaBi because Dowthern A was not available to use.

- $\quad$ Truck in liquid form (CargoHandbook, 2014)

\section{C.4 Methanol}

Details/Assumptions:

- $\quad$ Truck in liquid form in drums (CargoHandbook, 2014)

- As GaBi database does not include methanol, transport of NG by truck is included to approximate the environmental impact. 
C.5 Hexane

Details/Assumptions:

- Normally transported as liquid in drums (CargoHandbook, 2014)

\section{C.6 Nitrogen gas}

Details/Assumptions:

- Normally transported as liquid (Air Products and Chemicals Inc., 2015)

- Majority recirculated, top up required /hr

C.7 Carbon dioxide

Details/Assumptions:

- Pipeline with 0 incline chosen in GaBi 
Appendix D - Life Cycle Impact Assessment Tables and Assumptions

D.1 PBR Life Cycle Impact Assessment Table, graph and assumptions

Table D.1: GHG impact associated with the PBR (scaling factor of 0.0078 is equivalent to a $100 \mathrm{~km}$ drive)

\begin{tabular}{|c|c|c|c|c|}
\hline $\begin{array}{l}\text { Input } \\
\text { /Output }\end{array}$ & Material / Service & Total value/hr & Value (scaling) & $\mathrm{CO}_{2} \mathrm{e}$ contribution \\
\hline Input & $\begin{array}{l}\text { Carbon dioxide } \\
\text { transport }\end{array}$ & $1,643 \mathrm{~kg}$ & $12.8 \mathrm{~kg}$ & $6.21 \mathrm{kgCO}_{2} \mathrm{e}$ \\
\hline Input & Wastewater & $4.79 \times 10^{\wedge} 6 \mathrm{~L}$ & $\mathrm{n} / \mathrm{a}$ & No impact \\
\hline Input & $\begin{array}{l}\text { Operational electrical } \\
\text { energy }\end{array}$ & $3,875 \mathrm{kWh}$ & & \\
\hline Input & $\begin{array}{l}\text { Electrical circulation } \\
\text { energy to/from } \operatorname{PBR}(\mathrm{s})\end{array}$ & $1,039 \mathrm{kWh}$ & & \\
\hline \multirow[t]{2}{*}{ Output } & $\begin{array}{l}\text { Electrical energy credit } \\
\text { from energy production }\end{array}$ & $-325 \mathrm{kWh}$ & & \\
\hline & Total electrical energy & $4,589 \mathrm{kWh}$ & $35.8 \mathrm{kWh}$ & $21.96 \mathrm{kgCO}_{2} \mathrm{e}$ \\
\hline Output & Microalgae & $1.27 \times 10^{\wedge} 3 \mathrm{~kg}$ & $9.91 \mathrm{~kg}$ & No impact \\
\hline Output & $\begin{array}{l}\text { Fertilizer credit } \\
\text { (ammonia and di- } \\
\text { ammonium phosphate) }\end{array}$ & $2.27 \mathrm{~kg}$ & & $-0.05 \mathrm{kgCO}_{2} \mathrm{e}$ \\
\hline \multicolumn{4}{|c|}{ Total impact } & $28.12 \mathrm{kgCO}_{2} \mathrm{e}$ \\
\hline
\end{tabular}

Details/Assumptions:

- No additional fertilizer is required as all $\mathrm{N}$ and $\mathrm{P}$ are recirculated to the point where there will be additional fertilizer credits (just over 48 hours operation)

- DAP (di-ammonium phosphate), the world's most widely used phosphorus fertilizer as well as ammonia to supplement nitrogen requirements (Williams \& Laurens, 2010) 
- This study did not include the burning of biofuel $\mathrm{CO}_{2} \mathrm{e}$ impact, as this same amount of carbon was sequestered in order to create the microalgae. Both values would simply cancel out.

- Not able to change aggregated impact values from DE (Denmark) data to US data as the US values are not available to select.

- This study used the LCIA tab in GaBi database for climate change impact values. These values are the same values found in other tabs (ReCiPe model) that use the GWP 100 model.

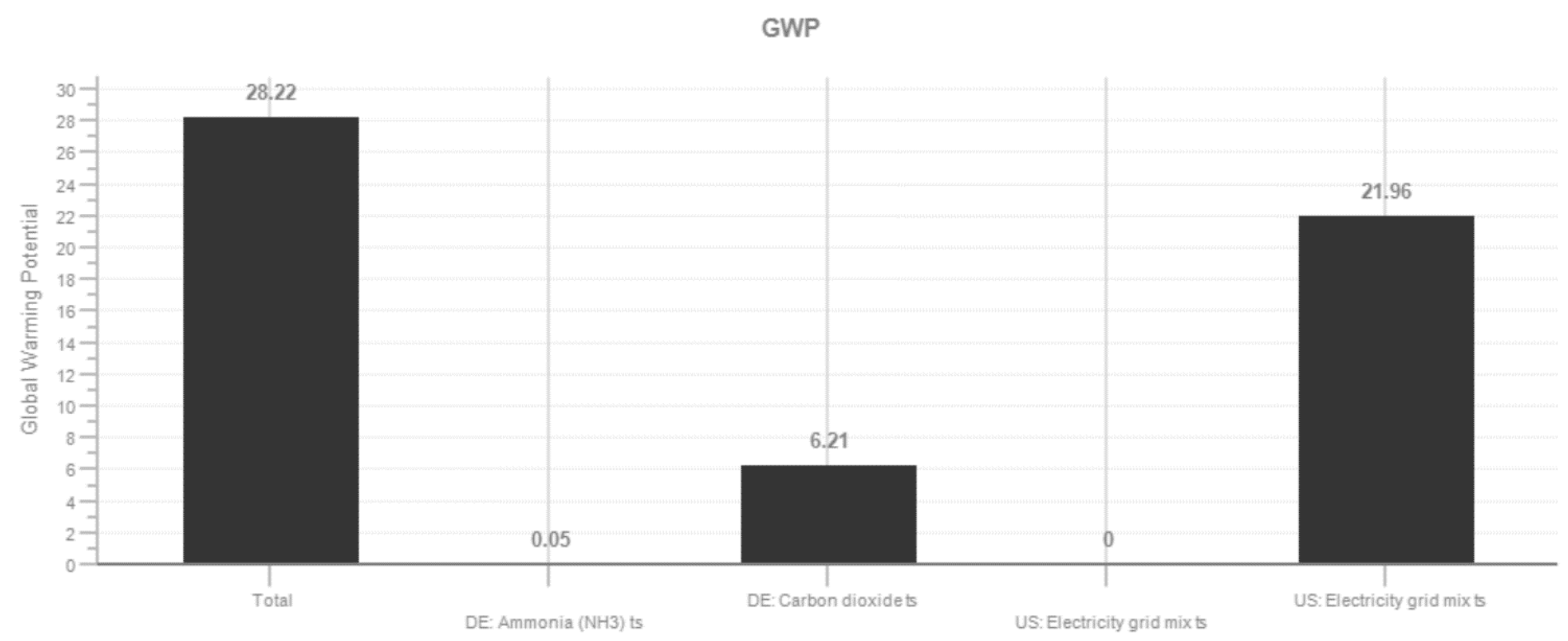

Figure D.1: Global Warming Potential of PBR process requirements (GaBi Thinkstep - PE International, 2017) 
D.2 Harvest Life Cycle Impact Assessment Table, graph and assumptions

Table D.2: GHG impact associated with the Harvest process: flocculation to dryer (scaling factor of 0.0078 equivalent to $100 \mathrm{~km}$ drive)

\begin{tabular}{|c|c|c|c|c|}
\hline $\begin{array}{l}\text { Input } \\
\text { /Output }\end{array}$ & Material / Service & Total value/hr & Value (scaling) & $\mathrm{CO}_{2} \mathrm{e}$ contribution \\
\hline \multirow[t]{2}{*}{ Input } & Sodium hydroxide & $958 \mathrm{~kg}$ & $7.47 \mathrm{~kg}$ & $9.53 \mathrm{kgCO}_{2} \mathrm{e}$ \\
\hline & $\begin{array}{l}\text { Transportation of } \\
\text { sodium hydroxide }\end{array}$ & $27 \mathrm{~km}$ & & inconsequential \\
\hline \multirow[t]{2}{*}{ Input } & Sulfric acid & $1,177 \mathrm{~kg}$ & $9.18 \mathrm{~kg}$ & $2.55 \mathrm{kgCO}_{2} \mathrm{e}$ \\
\hline & $\begin{array}{l}\text { Transportation of } \\
\text { sulfuric acid }\end{array}$ & $27 \mathrm{~km}$ & & inconsequential \\
\hline Input & $\begin{array}{l}\text { Floatation and } \\
\text { flocculation electrical } \\
\text { energy }\end{array}$ & $72 \mathrm{kWh}$ & & \\
\hline Input & $\begin{array}{l}\text { Centrifuge electrical } \\
\text { energy }\end{array}$ & $5,450 \mathrm{kWh}$ & & \\
\hline Input & $\begin{array}{l}\text { Electrical energy for } \\
\text { material circulation }\end{array}$ & $3.3 \mathrm{kWh}$ & & \\
\hline \multirow[t]{2}{*}{ Output } & $\begin{array}{l}\text { Electrical energy credit } \\
\text { from energy production }\end{array}$ & $-369 \mathrm{kWh}$ & & \\
\hline & Total electrical energy & $5,156 \mathrm{kWh}$ & $40.3 \mathrm{kWh}$ & $24.68 \mathrm{kgCO}_{2} \mathrm{e}$ \\
\hline Input & Dryer energy $(\mathrm{NG})$ & $\begin{array}{l}4,053 \mathrm{kWh}(310 \\
\mathrm{kg})\end{array}$ & $2.42 \mathrm{~kg}$ & $1.11 \mathrm{kgCO}_{2} \mathrm{e}$ \\
\hline Input & $\begin{array}{l}\text { Additional wastewater } \\
\text { created }\end{array}$ & $432 \mathrm{~kg}$ & $3.37 \mathrm{~kg}$ & 0.08 \\
\hline \multicolumn{4}{|c|}{ Total Impact } & $37.95 \mathrm{kgCO}_{2} \mathrm{e}$ \\
\hline
\end{tabular}

Details/Assumptions: 
- This study assumed $100 \%$ microalgae recovery

- This study assumes that NG arrives at plant via existing underground pipeline

- $\quad$ See Appendix C for transport calculations and references

- Amount of NG required is calculated using LHV of methane (47 MJ/kg)

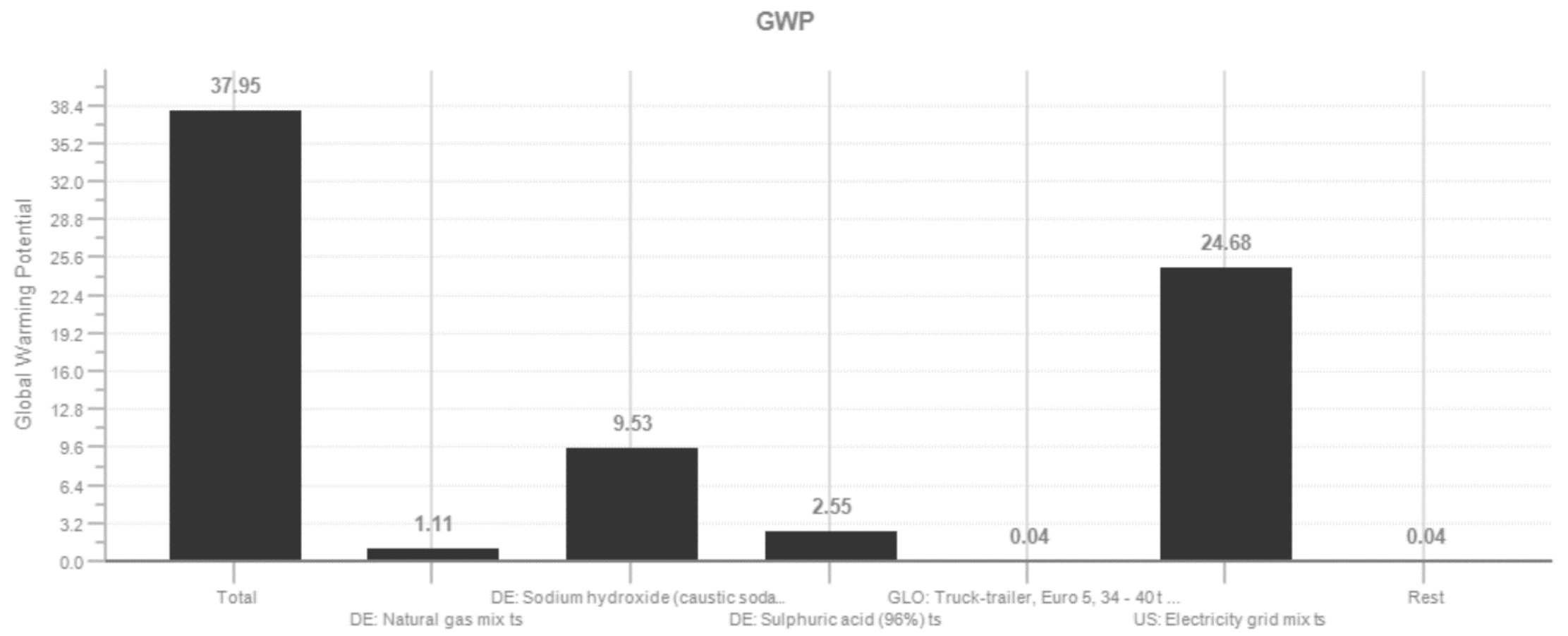

Figure D.2: Global Warming Potential of Harvesting process requirements (GaBi Thinkstep - PE International, 2017) 
D.3 Separation Life Cycle Impact Assessment Table, graph and assumptions

Table D.3: GHG impact associated with the Separation process: pulverizing to condensing of solvents for recovery (scaling factor is 0.0078 equivalent to $100 \mathrm{~km}$ driven)

\begin{tabular}{|c|c|c|c|c|}
\hline $\begin{array}{l}\text { Input } \\
\text { /Output }\end{array}$ & Material / Service & Total value/hr & Value (scaling) & $\mathrm{CO}_{2} \mathrm{e}$ contribution \\
\hline Input & $\begin{array}{l}\text { Pulverizing electrical } \\
\text { energy }\end{array}$ & $67 \mathrm{kWh}$ & & \\
\hline Input & CSTR electrical energy & $184.84 \mathrm{kWh}$ & & \\
\hline Input & $\begin{array}{l}\text { Centrifuge electrical } \\
\text { energy }\end{array}$ & $20 \mathrm{kWh}$ & & \\
\hline Input & $\begin{array}{l}\text { Electrical energy for } \\
\text { material circulation }\end{array}$ & $2.5 \mathrm{kWh}$ & & \\
\hline \multirow[t]{2}{*}{ Output } & $\begin{array}{l}\text { Electrical energy credit } \\
\text { from energy production }\end{array}$ & $-22 \mathrm{kWh}$ & & \\
\hline & Electrical requirement & $252 \mathrm{kWh}$ & $1.97 \mathrm{kWh}$ & $1.21 \mathrm{kgCO}_{2} \mathrm{e}$ \\
\hline \multirow[t]{2}{*}{ Input } & Hexane & $5.99 \mathrm{~kg}$ & $0.047 \mathrm{~kg}$ & $0.1 \mathrm{kgCO}_{2} \mathrm{e}$ \\
\hline & $\begin{array}{l}\text { Transportation of } \\
\text { hexane }\end{array}$ & $27 \mathrm{~km}$ & & inconsequential \\
\hline \multirow[t]{2}{*}{ Input } & Ethanol & $0.65 \mathrm{~kg}$ & & none \\
\hline & $\begin{array}{l}\text { Transportation of } \\
\text { ethanol }\end{array}$ & Use ethanol on site & & \\
\hline Input & CSTR heat (NG) & $874 \mathrm{kWh}$ & & \\
\hline Input & $\begin{array}{l}\text { Evaporation energy } \\
\text { (NG) }\end{array}$ & $1,046 \mathrm{kWh}$ & & \\
\hline \multirow[t]{2}{*}{ Input } & $\begin{array}{l}\text { Energy for heating } \\
\text { /cooling fluid } \\
\text { circulation }(\mathrm{NG})\end{array}$ & $0.256 \mathrm{kWh}$ & & \\
\hline & Total NG requirement & $\begin{array}{l}\text { 1,920 kWh (145.7 } \\
\text { kg) }\end{array}$ & $1.14 \mathrm{~kg}$ & $0.71 \mathrm{kgCO}_{2} \mathrm{e}$ \\
\hline \multicolumn{4}{|c|}{ Total Impacts } & $2.02 \mathrm{kgCO}_{2} \mathrm{e}$ \\
\hline
\end{tabular}


Details/Assumptions:

- This study assumes that NG arrives at plant via existing underground pipeline

- $\quad$ See Appendix C for transport calculations and references

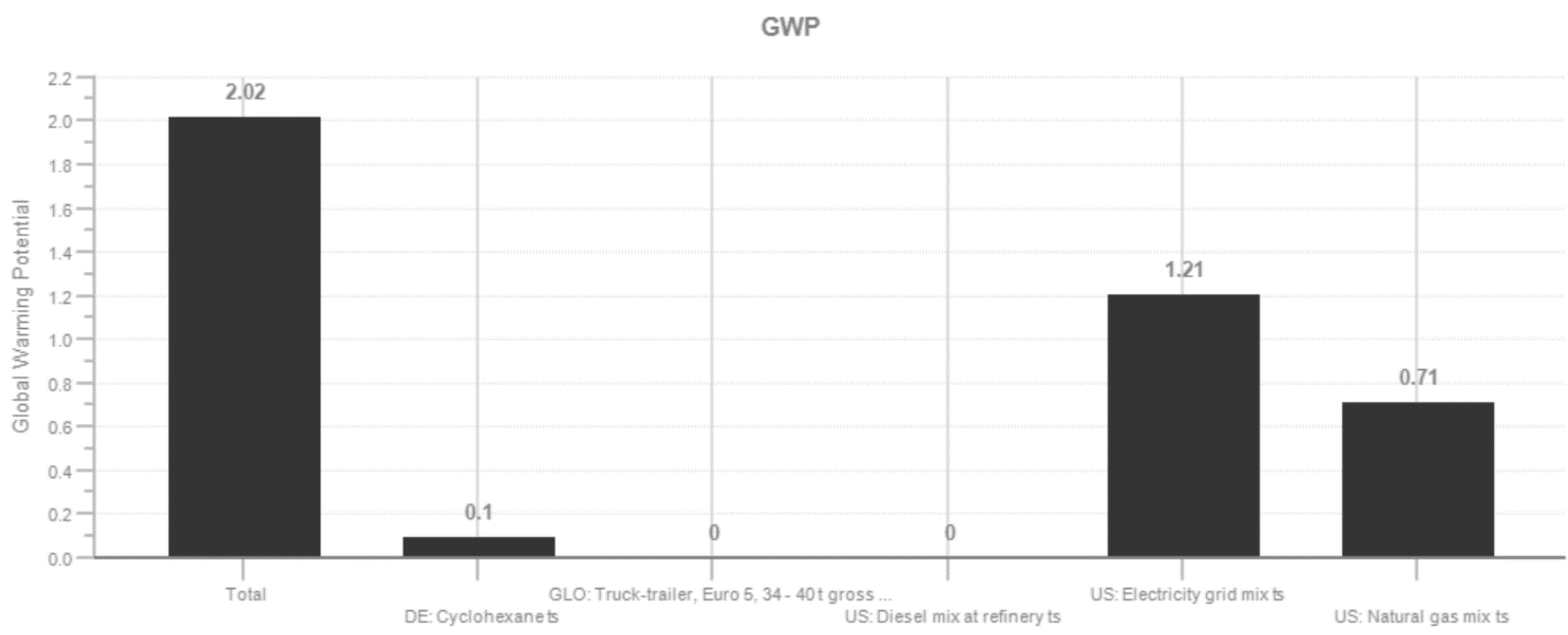

Figure D.3: Global Warming Potential of Separation process requirements (GaBi Thinkstep - PE International, 2017) 
D.4 BD Life Cycle Impact Assessment Table, graph and assumptions

Table D.4: GHG impact associated with the Biodiesel production process (scaling factor is 0.0078 equivalent to $100 \mathrm{~km}$ driven)

\begin{tabular}{|c|c|c|c|c|}
\hline $\begin{array}{l}\text { Input } \\
\text { /Output }\end{array}$ & Material / Service & Total value/hr & Value (scaling) & $\mathrm{CO}_{2} \mathrm{e}$ contribution \\
\hline Input & $\begin{array}{l}\text { Compressor electrical } \\
\text { energy }\end{array}$ & $3.6 \mathrm{kWh}$ & & \\
\hline Input & $\begin{array}{l}\text { Electrical energy for } \\
\text { material circulation }\end{array}$ & $4.3 \mathrm{kWh}$ & & \\
\hline \multirow[t]{2}{*}{ Input } & $\begin{array}{l}\text { Centrifuge electrical } \\
\text { energy }\end{array}$ & $0.746 \mathrm{kWh}$ & & \\
\hline & Total electrical energy & $8.6 \mathrm{kWh}$ & $0.067 \mathrm{kWh}$ & $0.04 \mathrm{kgCO}_{2} \mathrm{e}$ \\
\hline \multirow[t]{2}{*}{ Input } & Dowthern A & $165.6 \mathrm{~kg}$ & $1.29 \mathrm{~kg}$ & $1.43 \mathrm{kgCO}_{2} \mathrm{e}$ \\
\hline & Transport of materials & $27 \mathrm{~km}$ & & $0.01 \mathrm{kgCO}_{2} \mathrm{e}$ \\
\hline Input & Methanol & $49.86 \mathrm{~kg}$ & $0.23 \mathrm{~kg}$ & $0.14 \mathrm{kgCO}_{2} \mathrm{e}$ \\
\hline Input & CSTR heat energy (NG) & $42.3 \mathrm{kWh}$ & & \\
\hline Input & $\begin{array}{l}\text { Evaporation energy } \\
\text { (NG) }\end{array}$ & $73 \mathrm{kWh}$ & & \\
\hline \multirow[t]{2}{*}{ Input } & $\begin{array}{l}\text { Energy for heating/ } \\
\text { cooling fluid circulation } \\
\text { (NG) }\end{array}$ & $0.147 \mathrm{kWh}$ & & \\
\hline & $\begin{array}{l}\text { Total NG } \\
\text { requirements }\end{array}$ & $115.4 \mathrm{kWh}$ & $0.9 \mathrm{kWh}$ & $0.23 \mathrm{kgCO}_{2} \mathrm{e}$ \\
\hline Input & $\begin{array}{l}\text { Transport of BD to } \\
\text { refinery or service } \\
\text { stations }\end{array}$ & $27 \mathrm{~km}$ & & $\begin{array}{l}\text { Included in Table } \\
3.2\end{array}$ \\
\hline \multicolumn{4}{|c|}{ Total Impact } & $1.86 \mathrm{kgCO}_{2} \mathrm{e}$ \\
\hline
\end{tabular}

Details/Assumptions:

- Product output values are found in Appendix A 
- This study assumes that NG arrives at plant via existing underground pipeline

- See Appendix C for transport calculations and references

- Methanol is substituted for NG in GaBi. Methanol:NG ratio is $1.7: 1$ (with density of $\mathrm{NG}$ at $25^{\circ} \mathrm{C}$ and 1 atm at $0.66 \mathrm{~g} / \mathrm{L}$ ) (Aasberg-Petersen et al., 2009)

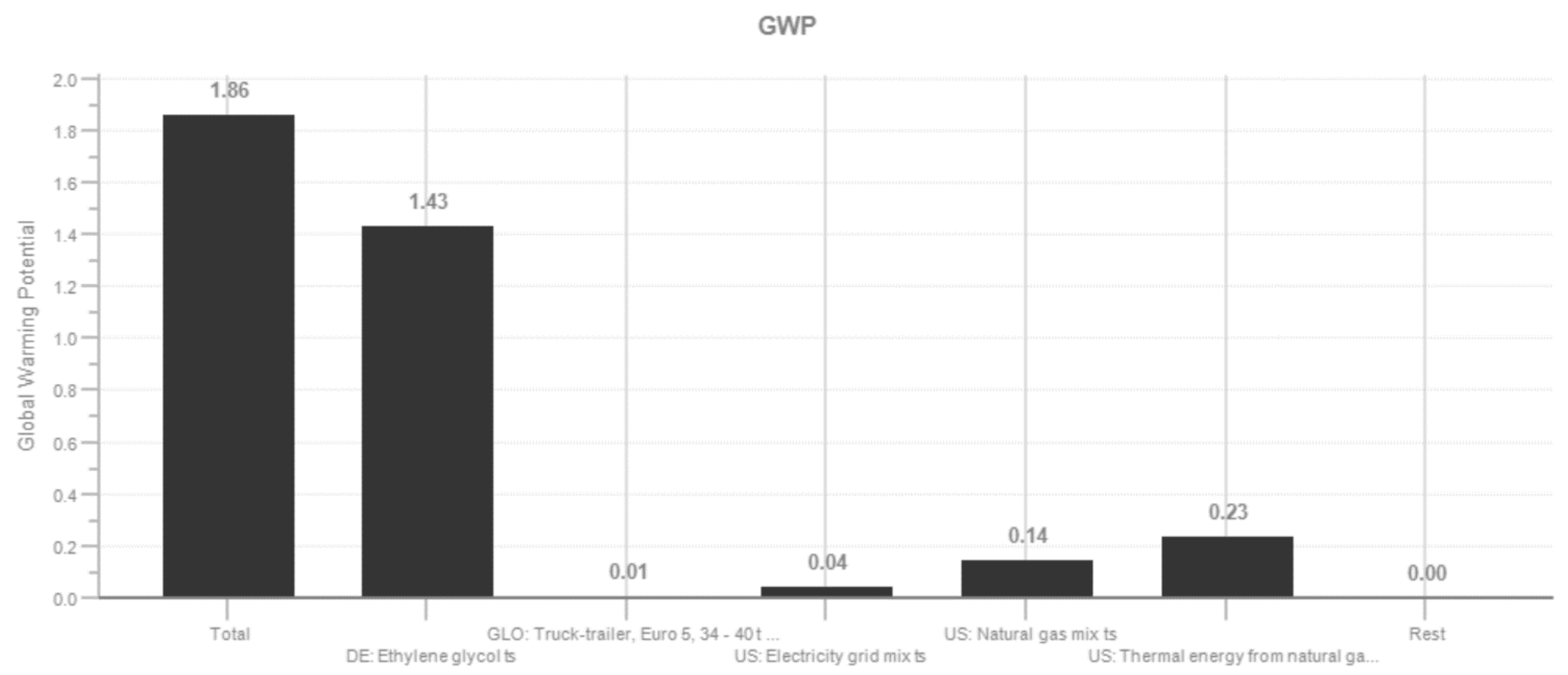

Figure D.4: Global Warming Potential of BD process requirements (GaBi Thinkstep - PE International, 2017) 
D.5 ABE Life Cycle Impact Assessment Table, graph and assumptions

Table D.5: GHG impact for ABE production process (scaling factor is 0.0078 equivalent to $100 \mathrm{~km}$ drive)

\begin{tabular}{|c|c|c|c|c|}
\hline $\begin{array}{l}\text { Input } \\
\text { /Output }\end{array}$ & Material / Service & Total value/hr & Value (scaling) & $\mathrm{CO}_{2} \mathrm{e}$ contribution \\
\hline Input & Sulfuric acid & $175 \mathrm{~kg}$ & $1.37 \mathrm{~kg}$ & $0.38 \mathrm{kgCO}_{2} \mathrm{e}$ \\
\hline Input & Water & $9,574 \mathrm{~kg}$ & $76 \mathrm{~kg}$ & $0.31 \mathrm{kgCO}_{2} \mathrm{e}$ \\
\hline Input & Heating (NG) & $1,160 \mathrm{kWh}$ & & \\
\hline Input & Evaporator heat (NG) & $14 \mathrm{kWh}$ & & \\
\hline Input & $\begin{array}{l}\text { Energy for } \\
\text { heating/cooling fluid } \\
\text { circulation }(\mathrm{NG})\end{array}$ & $\begin{array}{l}\text { In BD section } \\
\text { (minimal) }\end{array}$ & & \\
\hline & Total power from NG & 1,174 kWh & $9.16 \mathrm{kWh}$ & $2.39 \mathrm{kgCO}_{2} \mathrm{e}$ \\
\hline Input & Sodium hydroxide & $165 \mathrm{~kg}$ & $1.29 \mathrm{kWh}$ & $1.64 \mathrm{kgCO}_{2} \mathrm{e}$ \\
\hline Input & Nitrogen & $142 \mathrm{~kg}$ & $1.11 \mathrm{~kg}$ & $0.25 \mathrm{kgCO}_{2} \mathrm{e}$ \\
\hline Input & $\begin{array}{l}\text { Electrical energy to } \\
\text { circulate nitrogen gas }\end{array}$ & $0.024 \mathrm{kWh}$ & & \\
\hline Input & $\begin{array}{l}\text { Electrical energy to } \\
\text { strip nitrogen gas from } \\
\text { SSF exhaust }\end{array}$ & $0.34 \mathrm{kWh}$ & & \\
\hline Input & $\begin{array}{l}\text { Electrical energy to } \\
\text { circulate evaporator } \\
\text { water }\end{array}$ & $6.4 \times 10^{\wedge}-3 \mathrm{kWh}$ & & \\
\hline Input & $\begin{array}{l}\text { Electrical energy for } \\
\text { material circulation }\end{array}$ & $4.7 \mathrm{kWh}$ & & \\
\hline & Total electrical power & $5 \mathrm{kWh}$ & $0.039 \mathrm{kWh}$ & $0.1 \mathrm{kgCO}_{2} \mathrm{e}$ \\
\hline Input & $\begin{array}{l}\text { Transport of products } \\
\text { (ABE) to refinery or } \\
\text { service station }\end{array}$ & 27 km & & $\begin{array}{l}\text { Included in Table } \\
3.2\end{array}$ \\
\hline Input & Evaporator water & $174 \mathrm{~kg}$ & $\begin{array}{l}\text { Included in water } \\
\text { above }\end{array}$ & \\
\hline
\end{tabular}




\begin{tabular}{|c|c|c|c|c|}
\hline \multirow{2}{*}{\multicolumn{4}{|c|}{$\begin{array}{l}\text { Output } \\
\text { Total Impact }\end{array}$}} & $0.03 \mathrm{kgCO}_{2} \mathrm{e}$ \\
\hline & & & & $5.1 \mathrm{kgCO}_{2} \mathrm{e}$ \\
\hline
\end{tabular}

Details/Assumptions:

- Product output values are found in Appendix A

- This study assumes that NG arrives at plant via existing underground pipeline

- See Appendix C for transport calculations and references

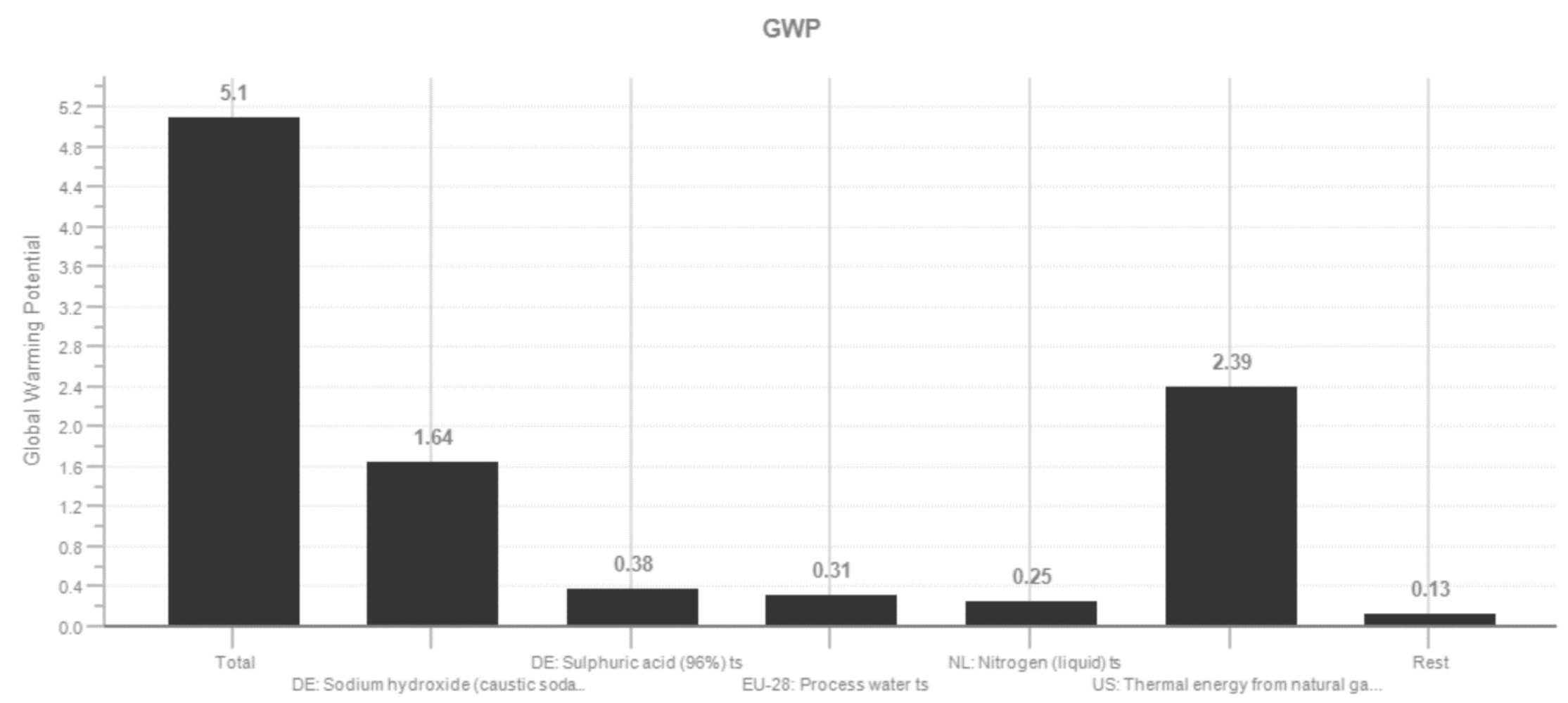

Figure D.5: Global Warming Potential of ABE process requirements (GaBi Thinkstep - PE International, 2017) 
D.6 AD and Power Life Cycle Impact Assessment Table, graph and assumptions

Table D.6: GHG impact of AD and power production process (scaling factor is 0.0078 equivalent to a $100 \mathrm{~km}$ drive)

\begin{tabular}{|l|l|l|l|l|}
\hline $\begin{array}{l}\text { Input } \\
\text { /Output }\end{array}$ & Material / Service & Total value/hr & Value (scaling) & CO2e contribution \\
\hline Input & $\begin{array}{l}\text { Electrical energy for } \\
\text { AD operation }\end{array}$ & $53 \mathrm{kWh}$ & & \\
\hline Input & $\begin{array}{l}\text { Electrical energy for } \\
\text { biogas stripping }\end{array}$ & $80 \mathrm{kWh}$ & & \\
\hline Output & $\begin{array}{l}\text { Electrical energy credit } \\
\text { from energy production }\end{array}$ & $-7 \mathrm{kWh}$ & & $0.6 \mathrm{kgCO}_{2} \mathrm{e}$ \\
\hline & Total electrical energy & $\mathbf{1 2 6} \mathbf{~ k W h}$ & $1 \mathrm{kWh}$ & \\
\hline
\end{tabular}

D.7 Scaling Factor Calculations

Bio-butanol energy produced: $33.1 \mathrm{MJ} / \mathrm{kg} \cdot 101 \mathrm{~kg} / \mathrm{hr}=3,343.1 \mathrm{MJ} / \mathrm{hr}$

Bio-ethanol energy: $26.8 \mathrm{MJ} / \mathrm{kg} \cdot(16.9-0.65 \mathrm{~kg}) / \mathrm{hr}=435.5 \mathrm{MJ} / \mathrm{hr}$

Bio-acetone energy: $29.6 \mathrm{MJ} / \mathrm{kg} \cdot 50.8 \mathrm{~kg} / \mathrm{hr}=1,503.12 \mathrm{MJ} / \mathrm{hr}$

FAME energy: $41 \mathrm{MJ} / \mathrm{kg} \cdot 430 \mathrm{~kg} / \mathrm{hr}=18,163 \mathrm{MJ} / \mathrm{hr}$

Total Energy produced: $23,445 \mathrm{MJ} / \mathbf{h r}(6,513 \mathrm{kWh})$

Scaling factor for $\mathrm{GaBi}: 183 \mathrm{MJ} / 23,445 \mathrm{MJ} / \mathrm{hr}=0.0078$ 
Appendix E - Comparison with other studies (calculations)

E.1 Comparison with Frank et al. (2011) study calculations

E.1.1 Difference between power requirements associated with cultivation + flocculation

7,375 Btu/kg-lipid (assuming lipid = oil) for cultivation and first dewatering (flocculation) (Frank et al. 2011)

This study 4,986 kWh PBR + $72 \mathrm{kWh}($ floc $)=4,986 \mathrm{kWh} / 443 \mathrm{~kg}$-oil = $11.26 \mathrm{kWh} / \mathrm{kg}$-oil

$11.26 \mathrm{kWh} / \mathrm{kg}$-oil = 38,101 Btu/kg-oil

$38,101 \mathrm{Btu} / \mathrm{kg}$-oil/ 7,375 Btu/kg-oil = 5

$\therefore$ 5x more power required for the cultivation and flocculation stages for this study than Frank et al. (2011)

\section{E.1.2 Difference in flocculation energy}

This study used $0.015 \mathrm{kWh} / \mathrm{m}^{3}$ of water

Frank et al. (2011) used $1.33 \times 10^{\wedge}-4 \mathrm{kWh} / \mathrm{dry}-\mathrm{g}$ microalgae

Total flocculation energy required for this study $=\mathbf{7 2} \mathbf{k W h}$

Using $1.33 \times 10^{\wedge}-4 \mathrm{kWh} / \mathrm{dry}-\mathrm{g}$ for this study:

$1.33 \times 10^{\wedge}-4 \mathrm{kWh} /$ dry-g $\cdot 1,266,000 \mathrm{dry}-\mathrm{g} / \mathrm{hr}=\mathbf{1 6 8 . 4} \mathbf{~ k W h}$

$\therefore$ more flocculation energy required for Frank et al. (2011) study 


\section{E.1.3 Difference in centrifuge energy calculations}

Difference in centrifuge power requirements:

*the term oil $=$ lipid in this section

$1.2 \mathrm{kWh} / \mathrm{m}^{3}$ for this study produced 5,450 kWh power requirement for $443 \mathrm{~kg}$-oil (over the course of an hour)

Using Frank et al. (2011)'s centrifuge power requirements and this study's production of microalgae, this study would require slightly less power: $3.3 \times 10^{\wedge}-3 \mathrm{kWh} / \mathrm{g}$-algae (Frank et al. $\left.2011 \mathrm{pg} 22\right)=3.3 \mathrm{kWh} / \mathrm{kg}$ algae $\cdot 1,266 \mathrm{~kg}$ algae $/ \mathrm{hr}$ (this study's production rate) $=$ $4,178 \mathrm{kWh}$

$4,178 \mathrm{kWh} / 5,450 \mathrm{kWh} \cdot 100=76 \%$

$\therefore$ Using Frank et al. (2011) centrifuge power requirements, this study would have reduced its power requirement here by $23 \%$

E.1.4 Incongruence between Frank et al. (2011)'s centrifuge energy requirements and gross energy use for remaining dewatering

This study

$5,450 \mathrm{kWh} / 443 \mathrm{~kg}$-lipid $=12.3 \mathrm{kWh} / \mathrm{kg}$-lipid (this value is lower than $* *$ because of this study's higher percent lipid in microalgae at $35 \%)$

$12.3 \mathrm{kWh} / \mathrm{kg}$-lipid·3,412.14 Btu/kWh $=41,978 \mathrm{Btu} / \mathrm{kg}$-lipid

$\therefore 41,978 \mathrm{Btu} / \mathrm{kg}$-lipid is the power required to dewater this study's microalgae per kg-lipid

Frank et al. (2011) power requirements for remaining dewatering (which is the centrifuge operation) pg 42 is 3,036 Btu/kg-lipid

Considering Frank et al. (2011)'s percent lipid amount in microalgal biomass of $25 \%$, and considering the power requirement for the centrifuge provided in Frank et al. (2011) of $3.3 \mathrm{kWh} / \mathrm{kg}$-algae, the power required for remaining dewatering per $\mathrm{kg}$ lipid: 
$3.3 \mathrm{kWh} / \mathrm{kg}$-algae $1 \mathrm{~kg}$-algae$/ 0.25 \mathrm{~kg}$-lipid $=13.2 \mathrm{kWh} / \mathrm{kg}$-lipid $* *$

$13.2 \mathrm{kWh} / \mathrm{kg}$-lipid·3,412.14 Btu/kWh = 45,040 Btu/kg-lipid

45,040 Btu/kg-lipid $\neq 3,036 \mathrm{Btu} / \mathrm{kg}$-oil

E.1.5 Drying vs. wet processing (Homogenization)

30.4 tonnes $/$ day $=1.267$ tonnes $/ \mathrm{hr} \cdot 1$ ton $/ 0.907$ tonne $=1.396 \mathrm{ton} / \mathrm{hr}$

$365 \mathrm{kWh} / \mathrm{dry}$ ton for homogenation $\cdot 1.396 \mathrm{ton} / \mathrm{hr}=509.7 \mathrm{kWh}$

This study's process uses $4,053 \mathrm{kWh}$ to dry the same amount of microalgae

$\therefore$ Would save $3,543.3 \mathrm{kWh}$ by using homogenization instead of a dryer

\section{E.1.6 Power produced vs. power required}

Difference between power produced by co-gen and power required for the process:

14,620 Btu/kg-oil generated by combined heat and power (CHP) in Frank et al. (2011)

19,450 Btu/kg-oil required by total process (Frank et al. 2011)

14,620 Btu/kg-oil / 19,450 Btu/kg-oil = 75\% of the power required by Frank et al. (2011) system is generated internally by CHP $723 \mathrm{kWh}$ co-gen facility produces $/ 10,859 \mathrm{kWh}$ required by the process $=7 \%$ of the power required by this study's process is generated by this system's co-generation process

E.1.7 Different in total power requirements 
This study: $443 \mathrm{~kg}$ microalgal oil/hr requires a total on-site electricity requirement of $10,859 \mathrm{kWh}$

$10,859 \mathrm{kWh} / 443 \mathrm{~kg}$-oil $=24 \mathrm{kWh} / \mathrm{kg}$-oil

$24 \mathrm{kWh} / \mathrm{kg}$-oil *1MJ/0.28 kWh = 87.5 MJ*1,000,000 Btu/1055 MJ = 82,980 Btu/ kg-oil

82,980 Btu/ kg-oil (this study) / 19,450 Btu/kg-oil (Frank et al., 2011) = 4

$\therefore 4 \mathbf{x}$ more power required for this study per kg-oil produced

\section{E.1.8 Lignocellulosic power generation through digester instead of ABE}

$443.26 \mathrm{~kg}$ lignocellulosic biomass $/ \mathrm{hr} \cdot 0.37 \mathrm{~m}^{3}$ methane $/ \mathrm{kg}$ lignocellulosic biomass $=164 \mathrm{~m}^{3} / \mathrm{hr}$ $164 \mathrm{~m}^{3}$ methane $/ \mathrm{hr} \cdot 0.777 \mathrm{~kg}$ methane $/ \mathrm{m}^{3}=127.4 \mathrm{~kg}$ methane $/ \mathrm{hr}$

$127.4 \mathrm{~kg}$ methane $/ \mathrm{hr} \cdot 47.14 \mathrm{MJ} / \mathrm{kg}$ methane $=6,007.2 \mathrm{MJ} / \mathrm{hr}$

6007.2 MJ/hr / $443 \mathrm{~kg}$-oil = 13.5 MJ/hr·kg-oil

13.5 MJ/hr·kg-oil = 12,852.4 Btu/hr $\cdot \mathrm{kg}$-oil

Power production $(30 \%)$

12,852.4 Btu/hr·kg-oil $\cdot(0.3)=3,858 \mathrm{Btu} / \mathrm{hr} \cdot \mathrm{kg}$-oil

$\therefore$ Additional 3,858 Btu/hr·kg-oil produced in this study by sending lignocellulosic biomass to AD

Comparing energy generated in this study with the output generated in Frank et al. (2011)

$723 \mathrm{kWh}$ this study's co-gen facility produces $+(3,858 \mathrm{Btu} / \mathrm{hr} * \mathrm{~kg}$-oil produced directing lignocellulosic biomass to AD for this study) $=723 \mathrm{kWh} *(3,412 \mathrm{Btu} / 1 \mathrm{kWh}) *(1 / 443 \mathrm{~kg}$-oil $)+(3,858 \mathrm{Btu} / \mathrm{hr} * \mathrm{~kg}$-oil $)=9,426.6 \mathrm{Btu} / \mathrm{hr} * \mathrm{~kg}$-oil 
9,426.6 Btu/hr*kg-oil is how much power this study would produce if lignocellulosic biomass was added to the power production process

14,620 Btu/kg-oil generated by combined heat and power (CHP) in Frank et al. (2011)

9,426.6 Btu/hr*kg-oil / 14,620 Btu/kg-oil $* 100=64 \%$

$\therefore$ this study produced $36 \%$ less power from methane than did Frank et al. (2011)

*the hourly unit should not play a role in discrepancy here

E.2 Gallon of gasoline equivalent calculations

Amount of product produced per year in liters:

FAME

$430 \mathrm{~kg} / \mathrm{hr} / 0.864 \mathrm{~kg} / \mathrm{L}=498 \mathrm{~L} / \mathrm{hr} * 24 \mathrm{~h} /$ day $* 7$ days/week $* 47$ weeks/year $=3,929,722 \mathrm{~L} /$ year (in line with approximately 4 million liters stated in section 2.3.4)

Butanol

$101.52 \mathrm{~kg} / \mathrm{hr} / 0.81 \mathrm{~kg} / \mathrm{L}=125.3 \mathrm{~L} / \mathrm{hr} * 24 \mathrm{~h} /$ day*7 days/week $* 47$ weeks/year = 989,632 L/year

Ethanol

$16.92 \mathrm{~kg} / \mathrm{hr} / 0.79 \mathrm{~kg} / \mathrm{L}=21.42 \mathrm{~L} / \mathrm{hr} * 24 \mathrm{~h} /$ day*7 days/week*47 weeks/year $=169,114 \mathrm{~L} /$ year

Acetone

$50.76 \mathrm{~kg} / \mathrm{hr} / 0.784 \mathrm{~kg} / \mathrm{L}=64.7 \mathrm{~L} / \mathrm{hr} * 24 \mathrm{~h} /$ day*7 days $/$ week $* 47$ weeks $/$ year $=511,226 \mathrm{~L} /$ year

Equivalent MJ produced per year (using densities above and LHV of each): 
FAME: $1.39 \times 10^{\wedge} 8 \mathrm{MJ} / \mathrm{year}$ (using 10k tonnes of microalgal biomass)

Butanol: $2.65 \times 10^{\wedge} 7 \mathrm{MJ} / \mathrm{year}$ (using 10k tonnes of microalgal biomass)

Ethanol: $3.58 \times 10^{\wedge} 6 \mathrm{MJ} / \mathrm{year}$ (using 10k tonnes of microalgal biomass)

Acetone: $1.19 \times 10^{\wedge} 7 \mathrm{MJ} / \mathrm{year}$ (using $10 \mathrm{k}$ tonnes of microalgal biomass)

$\therefore$ Total energy produced $/$ year (using 10k tonnes of microalgal biomass) $=1.809 \times 10^{\wedge} 8 \mathrm{MJ}$

Verification check using MJ produced per hour from D.7.

23,445 MJ/hr*24h/day*7 days/week*47 weeks/year $=1.85 \times 10^{\wedge} 8 \mathrm{MJ}$

$$
1.85 \times 10^{\wedge} 8 \mathrm{MJ} \sim 1.809 \times 10^{\wedge} 8 \mathrm{MJ}
$$

Given:

- $\quad$ Gasoline LHV $=31.5 \mathrm{MJ} / \mathrm{kg}$

- Density of gasoline $=0.74 \mathrm{~kg} / \mathrm{L}$

- 1 gallon $=3.785$ liters

$31.5 \mathrm{MJ} / \mathrm{kg}$ gas $* 0.74 \mathrm{~kg}$ gas $/ \mathrm{L}=23.31 \mathrm{MJ} / \mathrm{L}$

23.31 MJ/L*3.785 L/gallon $=88.23 \mathrm{MJ} /$ gallon gas

$1.809 \mathrm{x} 10^{\wedge} 8 \mathrm{MJ}$ total produced (using 10k tonnes of microalgal biomass)/10,000 tonnes of microalgal biomass $=18,090 \mathrm{MJ}$ produced per tonne of microalgal biomass

18,090 MJ / tonne of microalgal biomass *0.907 tonne/ton $=16,408 \mathrm{MJ} /$ ton

16,408 MJ/ton / 88.23 MJ/gallon gas = 186 gallons/ton

$\therefore$ This study produced 186 gallons of gasoline equivalent /ton of microalgal biomass 


\section{References}

Aasberg-Petersen, K., Nielsen, C. S., Dybkjaer, I., \& Perregaard, J. (2009). Large Scale Methanol Production from Natural Gas. Ravnholm. (pp. 1-14).

Agarwal, A. K., \& Das, L. M. (2001). Biodiesel Development and Characterization for Use as a Fuel in Compression Ignition Engines. Journal of Engineering for Gas Turbines and Power, 123(2), 440-447.

Ağda $\breve{g}$, O. N., \& Sponza, D. T. (2007). Co-digestion of mixed industrial sludge with municipal solid wastes in anaerobic simulated landfilling bioreactors. Journal of Hazardous Materials, 140(1-2), 75-85.

Agriculture \& Agri-Food Canada. (2018). Greenhouse gases. Retrieved February 26, 2018, from http://www.agr.gc.ca/eng/science-and-innovation/agricultural-practices/agriculture-andclimate/greenhouse-gases/?id=1329321969842

Ahmad, F., Khan, A. U., \& Yasar, A. (2013). Transesterification of oil extracted from different species of algae for biodiesel production. African Journal of Environmental Science and Technology, 7(6), 358-364.

Air Products and Chemicals Inc. (2015). Liquid Nitrogen - Physical and Chemical Properties. Allentown. (pp. 1-8).

Al-Hasan, M. I. (2013). BIODIESEL PRODUCTION FROM WASTE FRYING OIL AND ITS APPLICATION TO A DIESEL ENGINE. Transport, 28(3), 276-289.

Al-Shamrani, A. A., James, A., \& Xiao, H. (2002). Separation of oil from water by dissolved air flotation. Colloids and Surfaces A: Physicochemical and Engineering Aspects, 209, 15-26.

Allan, J. D., Williams, P. I., Morgan, W. T., Martin, C. L., Flynn, M. J., Lee, J., ... Coe, H. (2010). Contributions from transport, solid fuel burning and cooking to primary organic aerosols in two UK cities. Atmospheric Chemistry and Physics, 10(2), 647-668.

Alsalme, A. (2008). Heteropoly acids as catalysts for liquid-phase esterification and transesterification. Applied Catalysis, 349(1-2), 170-176.

Amanor-Boadu, V., Pfromm, P. H., \& Nelson, R. (2014). Economic feasibility of algal biodiesel under alternative public policies. Renewable Energy, 67, 136-142.

Andersen, K., \& Kuhn, K. (2014). Cowspiracy. A.U.M. Films and First Spark Media. Retrieved from http://www.cowspiracy.com/about/

Anitescu, G., \& Bruno, T. (2012). Fluid properties needed in supercritical transesterification of triglyceride feedstocks to biodiesel fuels for efficient and clean combustion - a review. The Journal of Supercritical Fluids, 63, 133-149.

Arbor, A. (1986). Future of Transport Fuels: alcohol fuels (1st ed.). Ottawa: Canadian Research Index.

Archer Daniels Midland Co. (2018). Biodiesel. Retrieved March 9, 2018, from 
https://www.adm.com/products-services/fuel/biodiesel

Askeland, D., Fulay, F., \& Wright, W. (2011). Physical Properties. In The Science and Engineering of Materials (6th ed., pp. 641-672). New York: Thomson-Engineering.

Aslan, S., \& Kapdan, I. K. (2006). Batch kinetics of nitrogen and phosphorus removal from synthetic wastewater by algae. Ecological Engineering, 28(1), 64-70.

ASTM INTERNATIONAL. (2014). Petroleum Standards. Retrieved February 27, 2018, from https://www.astm.org/Standards/petroleum-standards.html

Ataya, F., Dubé, M. A., \& Ternan, M. (2007). Acid-Catalyzed Transesterification of Canola Oil to Biodiesel under Single- and Two-Phase Reaction Conditions. Energy Fuels, 21(4), 24502459.

Awudu, I., \& Zhang, J. (2011). Uncertainties and sustainability concepts in biofuel supply chain management: A review. Renewable and Sustainable Energy Reviews, 16, 1359-1368.

Azapagic, A., \& Clift, R. (1999). Allocation of environmental burdens in co-product systems: Product-related burdens (Part 1). The International Journal of Life Cycle Assessment, 4(6), 357-369.

Bai, X., Ghasemi Naghdi, F., Ye, L., Lant, P., \& Pratt, S. (2014). Enhanced lipid extraction from algae using free nitrous acid pretreatment. Bioresource Technology, 159, 36-40.

Bajpai, R., Prokop, A., \& Zappi, M. (Eds.). (2014). Algal Biorefineries (1st ed.). Dordrecht: Springer Netherlands.

Balaban, M., Soeder, C. J., \& Shelef, G. (1980). Algae biomass : production and use in collaboration with the National Council for Research and Development, Israël and the Gesellschaft für Strahlen und Umweltforschung (GSF). In G. Shelef \& C. J. Soeder (Eds.), International Symposium on the Production and Use of Micro-algae Biomass Acre (p. 852). Amsterdam: Elsevier/North-Holland Biomedical Press.

Balan, V. (2014). Current Challenges in Commercially Producing Biofuels from Lignocellulosic Biomass. ISRN Biotechnology. Lansing. (pp. 1-31).

Baliga, R., \& Powers, S. E. (2010). Sustainable Algae Biodiesel Production in Cold Climates. International Journal of Chemical Engineering, 2010, 1-13.

Bare, J. C., Hofstetter, P., Pennington, D. W., \& Udo De Haes, H. A. (2000). State-of-the-Art State-of-the-Art: LCIA Midpoints versus Endpoints: The Sacrifices and Benefits. International Journal of LCA, 5(6), 319-326.

Bare, J. C., Norris, G. A., Pennington, D. W., \& McKone, T. (2003). The Tool for the Reduction and Assessment of Chemical and Other Environmental Impacts. Journal of Industrial Ecology, 6(3-4), 49-78.

Barlow, J., Sims, R. C., \& Quinn, J. C. (2016). Techno-economic and life-cycle assessment of an attached growth algal biorefinery. Biosource Technology, 220, 360-368.

Barrie wastewater treatment facility. (2017). Private conversation with engineer of Barrie wastewater treatment facility. Barrie. 
Basf Petronas Chemicals. (2006). Technical Leaflet Petrochemicals: $n$-BUTANOL. Retrieved from http://www.solvents.basf.com/portal/load/fid245442/n-BUTANOL

Bauer, F., Hulteberg, C., Persson, T., \& Tamm, D. (2013). Biogas upgrading - Review of commercial technologies (Biogasuppgradering - Granskning av kommersiella tekniker); Catalyzing energy gas development for sustainable solutions. Malmo. (pp. 1-81).

Begum, S., \& Dahman, Y. (2015). Enhanced biobutanol production using novel clostridial fusants in simultaneous saccharification and fermentation of green renewable agriculture residues. Biofuels, Bioproducts and Biorefining, 9, 529-544.

Belay, A. (1997). Mass culture of Spirulina outdoors - the earthrise farms experience. In A. Vonshak (Ed.), Spirulina platensis ( Arthrospira ): physiology, cell-biology and biochemistry (pp. 131-158). London: Taylor \& Francis.

Bernal, J. M., Lozano, P., García-Verdugo, E., Burguete, M. I., Sánchez-Gómez, G., LópezLópez, G., ... Luis, S. V. (2012). Supercritical Synthesis of Biodiesel. Molecules, 17(12), 8696-8719.

Bernstein, L., Bosch, P., Canziani, O., Chen, Z., Christ, R., Davidson, O., ... Wratt, D. (2007). Climate Change 2007: Synthesis Report. Valencia. (pp. 1-52).

Bhola, V., Swalaha, F., Ranjith Kumar, R., Singh, M., \& Bux, F. (2014). Overview of the potential of microalgae for $\mathrm{CO} 2$ sequestration. International Journal of Environmental Science and Technology, 11(7), 2103-2118.

Biodiesel Magazine. (2017). The Latest News and Data About Biodiesel Production. Retrieved September 20, 2017, from http://www.biodieselmagazine.com/plants/listplants/Canada/existing/

Bligh, E. G., \& Dyer, W. J. (1959). A RAPID METHOD OF TOTAL LIPID EXTRACTION AND PURIFICATION. Canadian Journal of Biochemistry and Physiology, 37(8), 911917.

Borges, L., Morón-Villarreyes, J. A., D’Oca, M. G. M., \& Abreu, P. C. (2011). Effects of flocculants on lipid extraction and fatty acid composition of the microalgae Nannochloropsis oculata and Thalassiosira weissflogii. Biomass and Bioenergy, 35(10), 4449-4454.

Borowitzka, M. A. (2013). Energy from Microalgae: A Short History. In Borowitzka M. \& Moheimani N. (Eds.), Algae for Biofuels and Energy. Developments in Applied Phycology (1st ed., pp. 1-15). Dordrecht: Springer.

Borowitzka, M., Borowitzka, L. J., Borowitzkal, M. A., \& Moulton, T. P. (1984). Production and utilization of microalgae The mass culture of Dunaliella salina for fine chemicals : From laboratory to pilot plant. Hydrobiologia, 116/117, 115-134.

Bradley, T., Maga, D., \& Antón, S. (2015). Unified approach to Life Cycle Assessment between three unique algae biofuel facilities. Applied Energy, 154, 1052-1061.

Brennan, L., \& Owende, P. (2010). Biofuels from microalgae-A review of technologies for production, processing, and extractions of biofuels and co-products. Renewable and 
Sustainable Energy Reviews, 14(2), 557-577.

Brentner, L. B., Eckelman, M. J., \& Zimmerman, J. B. (2011). Combinatorial Life Cycle Assessment to Inform Process Design of Industrial Production of Algal Biodiesel. Environmental Science \& Technology, 45(16), 7060-7067.

Broch, A., Jena, U., Hoekman, S. K., \& Langford, J. (2014). Analysis of Solid and Aqueous Phase Products from Hydrothermal Carbonization of Whole and Lipid-Extracted Algae. Energies, 7, 62-79.

Bunker, D. Q., Edzwald, J. K., Dahlquist, J., \& Gillberg, L. (1995). Pretreatment considerations for dissolved air flotation: Water type, coagulants and flocculation. Water Science and Technology, 31(3-4), 63-71.

Burlew, J. S. (Ed.). (1953). ALGAL CULTURE FROM LABORATORY TO PILOT PLANT. Washington, D.C.: Carnegie Institution of Washington. (pp. 1-369).

Cabus-Llaurado, M. C., Cesteros, Y., Medina, F., Salagre, P., \& Sueiras, J. E. (2007). Omega 3 fatty acid ethyl ester from a simple catalytic non-oxidative dehydrogenation of a biobased oleochemical. Catalysis Communications, 8(3), 319-323.

Cameron, M. (2017, November). What if Ontario scrapped cap-and-trade for a carbon tax? Macleans. Retrieved from http://www.macleans.ca/opinion/what-if-ontario-scrapped-capand-trade-for-a-carbon-tax/

CargoHandbook. (2014). Category: Products. Retrieved February 21, 2018, from http://www.cargohandbook.com/index.php/Category:Products

Carter Day International Inc. (2012). Grinding Aquafeed With Jacobson Air Swept Pulverizers Quality Size Reduction Equipment. Retrieved from http://www.carterday.com/wpcontent/uploads/JacobsonPulverizerBrochure2012.pdf

Carvalho, A. P., Meireles, L. A., \& Malcata, F. X. (2006). Microalgal Reactors: A Review of Enclosed System Designs and Performances. Biotechnology Progress, 22(6), 1490-1506.

Castro, Y. A., Ellis, J. T., Miller, C. D., \& Sims, R. C. (2015). Optimization of wastewater microalgae saccharification using dilute acid hydrolysis for acetone, butanol, and ethanol fermentation. Applied Energy, 140, 14-19.

Cengel, Y. A., \& Boles, M. A. (2002). The second law of thermodynamics. In Thermodynamics: An Engineering Approach (4th ed.). New York: McGraw-Hill.

Chatsungnoen, T., \& Chisti, Y. (2016). Oil production by six microalgae: impact of flocculants and drying on oil recovery from the biomass. Journal of Applied Phycology, 28(5), 26972705.

Chen, C., Yeh, K., Aisyah, R., Lee, D., \& Chang, J. (2011). Cultivation, photobioreactor design and harvesting of microalgae for biodiesel production: A critical review. Bioresource Technology, 102(1), 71-81.

Chen, C., Zhao, X., Yen, H., Ho, S., Cheng, C., Lee, D., ... Chang, J. (2013). Microalgae-based carbohydrates for biofuel production. Biochemical Engineering Journal, 78, 1-10. 
Chen, G., Zhao, L., \& Qi, Y. (2015). Enhancing the productivity of microalgae cultivated in wastewater toward biofuel production: A critical review. Applied Energy, 137, 282-291.

Chen, J., Jo Zidwick, M., \& Rogers, P. (2013). Organic Acid and Solvent Production: Butanol, Acetone, and Isopropanol; 1,3-and 1,2-Propanediol Production; and 2,3-Butanediol Production. In The Prokaryotes - Applied Bacteriology and Biotechnology (pp. 78-125). Berlin: Springer-Verlang.

Chen, L., Liu, F., Liu, Y., Dong, H., \& Colberg, P. J. S. (2011). Benzene and toluene biodegradation down gradient of a zero-valent iron permeable reactive barrier. Journal of Hazardous Materials, 188, 110-115.

Cheng, W., \& Kung, H. H. (Eds.). (1994). Methanol production and use. (pp. 1-329). New York: Dekker.

Chisti, Y. (2007). Biodiesel from microalgae. Biotechnology Advances, 25, 294-306.

Choi, B. C. K., Pang, T., Lin, V., Puska, P., Sherman, G., Goddard, M., ... Clottey, C. (2005). Can scientists and policy makers work together? Journal of Epidemiology and Community Health, 59(8), 632-7.

Chorus, I., \& Bartram, J. (1999). Toxic Cyanobacteria in Water: A guide to their public health consequences, monitoring and management Determination of Organic Compounds in Natural and Treated Waters. Geneva. (pp. 1-400).

Christenson, L., \& Sims, R. (2011). Production and harvesting of microalgae for wastewater treatment, biofuels, and bioproducts. Biotechnology Advances, 29, 686-702.

City of Barrie. (2004). CITY OF BARRIE WATER POLLUTION CONTROL CENTRE. Barrie. Retrieved from http://www.barrie.ca/Living/Environment/Wastewater-AndSewers/Documents/WaterPollutionControlCentrePamphlet.pdf

City of Toronto. (2017). Geography - Toronto Facts - Your City | City of Toronto. Retrieved December 9, 2017, from https://www.toronto.ca/

Clarens, A. F., Nassau, H., Resurreccion, E. P., White, M. A., \& Colosi, L. M. (2011). Environmental Impacts of Algae-Derived Biodiesel and Bioelectricity for Transportation. Environment Science and Technology, 45, 7554-7560.

Clasen, C., Wilhelms, T., \& Kulicke, W.-M. (2006). Formation and Characterization of Chitosan Membranes. Biomacromolecules, (7), 3210-3222.

Cleantech Canada. (2014, April). Ontario rolls out new diesel regulations to curb emissions Canadian Manufacturing. Retrieved from https://www.canadianmanufacturing.com/sustainability/ontario-rolls-new-dieselregulations-curb-fuels-ghg-emissions-136340/

Cobb, J. (2015). California Is Smiling On Renewable Diesel HPR Fuel. Retrieved December 12, 2016, from http://www.hybridcars.com/california-is-smiling-on-renewable-diesel-hpr-fuel/

Collet, P., Hélias, A., Lardon, L., Ras, M., Goy, R.-A., \& Steyer, J.-P. (2011). Life-cycle assessment of microalgae culture coupled to biogas production. Bioresource Technology, 
$102,207-214$.

Collet, P., Lardon, L., Elias, A. H., Ephanie Bricout, S., Lombaert-Valot, I., Eatrice Perrier, B., ... Bernard, O. (2014). Biodiesel from microalgae - Life cycle assessment and recommendations for potential improvements. Renewable Energy, 71, 525-533.

Collotta, M., Busi, L., Champagne, P., Mabee, W., Tomasoni, G., \& Alberti, M. (2016). Evaluating microalgae-to-energy systems: different approaches to life cycle assessment (LCA) studies. Biofuels, Bioproducts and Biorefining, 10, 883-895.

Colman, B., \& Rotatore, C. (1995). Photosynthetic inorganic carbon uptake and accumulation in two marine diatoms. Plant, Cell and Environment, 18(8), 919-924.

Colosi, L. M. (2012). Will algae produce the green? Using published life cycle assessments as a starting point for economic evaluation of future algae-to-energy systems. Biofuels, 3(2), 129-142.

Conroy, J. D., Quinlan, E. L., Kane, D. D., \& Culver, D. A. (2007). Cylindrospermopsis in Lake Erie: Testing its Association with Other Cyanobacterial Genera and Major Limnological Parameters. Journal of Great Lakes Research, 33, 519-535.

Corriere, F., Peri, G., \& Rocca, V. La. (2013). Characterization of Biodiesel from Vegetable Oil Using Comprehensive Two-Dimensional Gas Chromatography. Applied Mechanics and Materials, 260-261, 312-317.

Cowichan Biodiesel Co-op. (2015). We Eat Locally, And So Do Our Cars! Retrieved March 9, 2018, from https://www.cowichanbiodiesel.org/

de Morais, M. G., \& Costa, J. A. V. (2007). Isolation and selection of microalgae from coal fired thermoelectric power plant for biofixation of carbon dioxide. Energy Conversion and Management, 48(7), 2169-2173.

Dolejš, I., Rebroš, M., \& Rosenberg, M. (2014). REVIEW Immobilisation of Clostridium spp. for production of solvents and organic acids. Chemical Papers, 68(1), 1-14.

Dong, T., Knoshaug, E. P., Davis, R., Laurens, L. M. L., Wychen, S. Van, Pienkos, P. T., \& Nagle, N. (2016). Combined algal processing: A novel integrated biorefinery process to produce algal biofuels and bioproducts. Algal Research, 19, 316-323.

Doucha, J., Straka, F., \& Lívanský, K. (2005). Utilization of flue gas for cultivation of microalgae Chlorella sp. in an outdoor open thin-layer photobioreactor. Journal of Applied Phycology, 17(5), 403-412.

Dragone, G., Fernandes, B., Vicente, A. A., \& Teixeira, J. A. (2010). Third generation biofuels from microalgae. In Applied Microbiology and Microbial Biotechnology (pp. 1355-1366). Saville: FORMATEX.

Ellis, J. T., Hengge, N. N., Sims, R. C., \& Miller, C. D. (2012). Acetone, butanol, and ethanol production from wastewater algae. Bioresource Technology, 111, 491-495.

Environment and Climate Change Canada. (2015). Environmental Indicators - Greenhouse Gas Emissions. Retrieved January 18, 2018, from https://www.ec.gc.ca/indicateurs- 
indicators/default.asp?lang=en\&n=FBF8455E-1

European Parliament and Council of the European Union. Directive on the promotion of the use of energy from renewable sources, Pub. L. No. 2009/28/EC (2009).

EVODOS. (2011). EVODOS type 25 centrifuge specifications. Raamsdonksveer. (pp. 1-8).

Farooq, W., Lee, Y.-C., Ryu, B.-G., Kim, B.-H., Kim, H.-S., Choi, Y.-E., \& Yang, J.-W. (2013). Two-stage cultivation of two Chlorella sp. strains by simultaneous treatment of brewery wastewater and maximizing lipid productivity. Bioresource Technology, 132, 230-238.

Farrar, W. V. (1966). Tecuitlatl; A Glimpse of Aztec Food Technology. Nature, 211(5047), 341342.

FEBICO (Far East Bio-Tech. Co.) Ltd. (2018). What is Microalgae. Retrieved March 18, 2018, from http://www.febico.com/en/health-library/What-is-Microalgae/FL-1-Microalgae.html

Féris, L. A., \& Rubio, J. (1999). Dissolved air flotation (DAF) performance at low saturation pressures. Filtration \& Separation, 36(9), 61-65.

Ferrell, J., \& Reed, S. V. (2010). National algal biofuels technology roadmap. College Park Maryland. (pp. 1-140).

Finnveden, G., Hauschild, M. Z., Ekvall, T., Guiné E D, J., Heijungs, R., Hellweg, S., ... Suh, S. (2009). Recent developments in Life Cycle Assessment. Journal of Environmental Management, 91, 1-21.

Fogler, S. H. (2006). Isothermal reactor design. In Elements of Chemical Reaction Engineering (4th ed., pp. 143-252). Westford: Pearson Education Inc.

Frank, E.D.; Han, J.; Palou-Rivera, I.; Elgowainy, A.; Wang, M. Q. (2011). Life-Cycle Analysis with Algal Lipid Fuels with the GREET model. Lemont. (pp. 1-87).

Frank, E. D., Elgowainy, A., Han, J., \& Wang, Z. (2013). Life cycle comparison of hydrothermal liquefaction and lipid extraction pathways to renewable diesel from algae. Mitigation and Adaptation Strategies for Global Change, 18(1), 137-158.

French, K., Guest, R. K., Finch, G. R., \& Haas, C. N. (2000). Correlating Cryptosporidium removal using dissolved air flotation in water treatment. Water Research, 34(16), 41164119.

Frigon, J. C., \& Guiot, S. R. (2010). Biomethane production from starch and lignocellulosic crops: A comparative review. Biofuels, Bioproducts and Biorefining, 4, 447-458.

GaBi Thinkstep - PE International. (2017). Life Cycle Assessment LCA Software: GaBi Software. GaBi Thinkstep. Retrieved from http://www.gabi-software.com/canada/index/

García-Serna, J., Pérez-Barrigón, L., \& Cocero, M. J. (2007). New trends for design towards sustainability in chemical engineering: Green engineering. Chemical Engineering Journal, 133(1-3), 7-30.

GEA Engineering. (2017). Flash dryers and coolers: Flash Dryer. Retrieved January 13, 2018, from https://www.gea.com/en/products/flash-dryer.jsp 
Geoghegan, M. J. (1951). Unicellular Algæ as a Source of Food. Nature, 168(4271), 426-427.

Giang, C., Bushati, K., \& Morgan-John, M. (2017). Algae Biorefinery. Ryerson University, Toronto.

Gibney, A. (2018). Dirty Money. Netflix. Retrieved from https://www.youtube.com/watch?v=CsplLiZHbj0

Ginzburg, B. (1993). Liquid fuel (oil) from halophilic algae: A renewable source of nonpolluting energy. Renewable Energy, 3(2-3), 249-252.

Gnansounou, E., \& Kenthorai Raman, J. (2016). Life cycle assessment of algae biodiesel and its co-products. Applied Energy, 161, 300-308.

Gnansounou, E., \& Pandey, A. (Eds.). (2017). Life-cycle assessment of biorefineries (1st ed.). Amsterdam, Kidlington \& Cambridge: Elsevier B.V.

Gómez, X., Cuetos, M. J., Cara, J., Morán, A., \& García, A. I. (2006). Anaerobic co-digestion of primary sludge and the fruit and vegetable fraction of the municipal solid wastes:

Conditions for mixing and evaluation of the organic loading rate. Renewable Energy, 31(12), 2017-2024.

Good, C., Andresen, I., \& Hestnes, A. G. (2015). SOLAR ENERGY FOR ZERO ENERGY BUILDINGS - A COMPARISON BETWEEN SOLAR THERMAL, PV AND PHOTOVOLTAIC-THERMAL (PV/T) SYSTEMS. Solar Energy, 122, 561-566.

Government of Canada. Renewable Fuels Regulations, Pub. L. No. SOR/2010-189 (2017). http://www.nrcan.gc.ca/energy/alternative-fuels/programs/12358.

Government of Canada. (2018). Sustainable Development Technology Canada. Retrieved March 18, 2018, from https://www.sdtc.ca/en

Graham, P. (2011). Towards establishing a sustainable aviation fuels industry in Australia and New Zealand: Sustainable aviation fuel road map. Canberra. (pp. 1-48).

Green, B. F., Bernstone, L. S., Lundquist, T. J., \& Oswald, W. J. (1996). Advanced integrated wastewater pond systems for nitrogen removal. Water Science and Technology, 33(7), 207217.

Green, E. M. (2011). Fermentative production of butanol - the industrial perspective. Current Opinion in Biotechnology, 22, 337-343.

Greque De Morais, M., \& Vieira Costa, J. A. (2007). Biofixation of carbon dioxide by Spirulina sp. and Scenedesmus obliquus cultivated in a three-stage serial tubular photobioreactor. Journal of Biotechnology, 129, 439-445.

Griffiths, E. (2009, December 1). Removal and Utilization of Wastewater Nutrients for Algae Biomass and Biofuels [Master's Thesis]. University archives. Utah State University.

Guan, G., Kusakabe, K., Sakurai, N., \& Moriyama, K. (2009). Transesterification of vegetable oil to biodiesel fuel using acid catalysts in the presence of dimethyl ether. Fuel, 88(1), 8186. 
Guston, D. H. (2001). Boundary Organizations in Environmental Policy and Science : An Introduction. Journal of Public Policy, 26(4), 399-408.

Haid, J., \& Koss, U. (2001). Lurgi's Mega-Methanol technology opens the door for a new era in down-stream applications. In E. Iglesia, J. . Spivey, \& T. H. Fleisch (Eds.), Studies in surface science and catalysts (136th ed., pp. 399-404). Elsevier.

Halim, R., Danquah, M. K., \& Webley, P. A. (2012). Extraction of oil from microalgae for biodiesel production: A review. Biotechnology Advances, 30, 709-732.

Hammer, S. K., \& Avalos, J. L. (2017). Harnessing yeast organelles for metabolic engineering. Nature Chemical Biology, 13(8), 823-832.

Hansen, A. C., Zhang, Q., \& Lyne, P. W. L. (2005). Ethanol-diesel fuel blends-a review. Biosource Technology, 96(3), 277-285.

Harari, Y. N. (2014). A Brief History of Humankind - Sapiens. McClelland \& Stewart (USA), Harvill Secker (UK).

Harder, R., \& von Witsch, H. (1942). Weitere Untersuchungen über die Veränderung der photoperiodischen Reaktion von Kalanchoe Blossfeldiana mit zunehmendem Alter der Pflanzen. Planta, 32(5), 547-557.

Harmsen, P. F. H., Huijgen, W. J. J., Bermúdez López, L. M., \& Bakker, R. R. C. (2010). Literature Review of Physical and Chemical Pretreatment Processes for Lignocellulosic Biomass. Wageningen. (pp. 1-49).

Harun, R., Davidson, M., Doyle, M., Gopiraj, R., Danquah, M., \& Forde, G. (2011). Technoeconomic analysis of an integrated microalgae photobioreactor, biodiesel and biogas production facility. Biomass and Bioenergy, 35, 741-747.

Hauschild, M. Z., Jeswiet, J., \& Alting, L. (2004). Design for Environment — Do We Get the Focus Right? CIRP Annals, 53(1), 1-4.

Hazlebeck, D. A., \& Dunlop, E. H. (2010, October 13). Photosynthetic oil production with high carbon dioxide utilization. U.S. Patent No. 7662616. United States of America.

Hira, A., \& Guilherme De Oliveira, L. (2009). No substitute for oil? How Brazil developed its ethanol industry. Energy Policy, 37(6), 2450-2456.

Hodaifa, G., Eugenia Martínez, M. A., \& Sánchez, S. (2007). Use of industrial wastewater from olive-oil extraction for biomass production of Scenedesmus obliquus. Bioresource Technology, 99(5), 1111-1117.

Hoffmeister, S., Gerdom, M., Bengelsdorf, F. R., Linder, S., Flüchter, S., Öztürk, H., ... Dürre, P. (2016). Acetone production with metabolically engineered strains of Acetobacterium woodii. Metabolic Engineering, 36, 37-47.

Honeywell UOP. (2018). Green Diesel v. Biodiesel. Retrieved March 13, 2018, from https://www.uop.com/processing-solutions/renewables/green-diesel/biodiesel/

Hooper, L. A., Hollein, H. C., \& Slater, C. S. (1998). Microfiltration of Streptomyces rimosus: Cell Harvesting Process Studies. Separation Science and Technology, 33(12), 1747-1765. 
Howard, W. L. (2011). Acetone. In Kirk-Othmer Encyclopedia of Chemical Technology (pp. 115). Hoboken, NJ, USA: John Wiley \& Sons, Inc.

Hu, Q., Sommerfeld, M., Jarvis, E., Ghirardi, M., Posewitz, M., Seibert, M., \& Darzins, A. (2008). Microalgal triacylglycerols as feedstocks for biofuel production: perspectives and advances. The Plant Journal, 54(4), 621-639.

Hu, W. (2014). Dry Weight and Cell Density of Individual Algal and Cyanobacterial Cells for Algae [Master's Thesis]. University of Missouri-Columbia.

Huber, G. W., \& Corma, A. (2007). Synergies between Bio-and Oil Refineries for the Production of Fuels from Biomass to Süd-Chemie on the occasion of its 150th anniversary. Angewandte Chemie International Edition, 46, 7184-7201.

Huertas, I. E., Colman, B., Espie, G. S., \& Lubian, L. M. (2001). Active transport of CO2 by three species of marine microalgae. Journal of Phycology, 36(2), 314-320.

Huesemann, M. H., Kuo, L., Urquhart, L., Gill, G. A., \& Roesijadi, G. (2012). Acetone-butanol fermentation of marine macroalgae. Bioresource Technology, 108, 305-309.

Hung, M. T., \& Liu, J. C. (2006). Microfiltration for separation of green algae from water. Colloids and Surfaces B: Biointerfaces, 51(2), 157-164.

Hunter, A. K. (2017). COMPARATIVE LIFE CYCLE ASSESSMENT: GROUND SOURCE HEAT PUMP SYSTEM VERSUS GAS FURNACE AND AIR CONDITIONER SYSTEM [Master's Project]. Ryerson University.

Igbokwe, J. O., \& Nwafor, O. M. I. (2014). Performance characteristics of palm kernel biodiesel and its blend in a CI engine. International Journal of Ambient Energy, 37(1), 103-106.

Independent Electricity System Operator. (2018). 18-Month Outlook: Ontario Electricity Generation System. Retrieved April 17, 2018, from http://www.ieso.ca/en/sectorparticipants/planning-and-forecasting/18-month-outlook

International Organization for Standardization. (2006a). ISO 14040 Life Cycle Assessment. Geneva. (pp. 1-20).

International Organization for Standardization. (2006b). ISO 14044 Environmental management - Life cycle assessment - Requirements and guidelines. Geneva. (pp. 1-46).

Jacobson, M. Z. (2009). Review of solutions to global warming, air pollution, and energy security. Energy Environmental Science, 2(2), 148-173.

Jingura, R. M., \& Matengaifa, R. (2009). Optimization of biogas production by anaerobic digestion for sustainable energy development in Zimbabwe. Renewable and Sustainable Energy Reviews, 13(5), 1116-1120.

Johnson, L., Kralovic, P., \& Romaniuk, A. (2016). CANADIAN CRUDE OIL AND NATURAL GAS PRODUCTION AND SUPPLY COSTS OUTLOOK. Calgary. (pp. 1-52).

Joint Research Centre for Environment and Sustainability. (2011). ILCD handbookRecommendations for Life Cycle Impact Assessment in the European Context. (pp. 1-159). 
Juguin, B. A., Boitiaux, J., \& Martino, G. J. (1990, December 19). Phenol production process. U.S. Patent No. 5160497. United States of America.

Kalnes, T. N., Koers, K. P., Marker, T., \& Shonnard, D. R. (2009). A technoeconomic and environmental life cycle comparison of green diesel to biodiesel and syndiesel. Environmental Progress \& Sustainable Energy, 28(1), 111-120.

Kapdi, S. S., Vijay, V. K., Rajesh, S. K., \& Prasad, R. (2005). Biogas scrubbing, compression and storage: perspective and prospectus in Indian context. Renewable Energy, 30(8), 11951202.

Karatay, S. E., \& Dönmez, G. (2011). Microbial oil production from thermophile cyanobacteria for biodiesel production. Applied Energy, 88(11), 3632-3635.

Kawaguchi, K. (1980). Microalgae production systems in Asia. In G. Shelef \& C. J. Soeder (Eds.), Algae biomass production and use (pp. 25-33). Amsterdam: Elsevier/ North Holland Biomedical Press.

Kelly, B. C., Ikonomou, M. G., Blair, J. D., Morin, A. E., \& Gobas, F. A. P. C. (2007). Food Web-Specific Biomagnification of Persistent Organic Pollutants. Source: Science, New Series, 317(5835), 236-239.

Kim, S., Ly, H. V., Kim, J., Lee, E. Y., \& Woo, H. C. (2015). Pyrolysis of microalgae residual biomass derived from Dunaliella tertiolecta after lipid extraction and carbohydrate saccharification. Chemical Engineering Journal, 263, 194-199. http://doi.org/10.1016/J.CEJ.2014.11.045

Kimantas, J. (2014). AJJ's 2014 environmental education guide: 112 canadian colleges and universities have more than 700 interdisciplinary programs in the study of humans and nature. Alternatives Journal, 40(4), 24-25.

Knothe, G. (2010). Biodiesel and renewable diesel: A comparison. Progress in Energy and Combustion Science, 36(3), 364-373.

Kök, M. S. (2016). An integrated approach: advances in the use of Clostridium for biofuel. Biotechnology and Genetic Engineering Reviews, 31(1-2), 69-81.

Kong, Q. X., Li, L., Martinez, B., Chen, P., \& Ruan, R. (2010). Culture of Microalgae Chlamydomonas reinhardtii in Wastewater for Biomass Feedstock Production. Applied Biochemistry and Biotechnology, 160, 9-18.

Kótai, L., Szépvölgyi, J., Szilágyi, M., Zhibin, L., Baiquan, C., Sharma, V., \& Sharma, P. K. (2013). Biobutanol from Renewable Agricultural and Lignocellulose Resources and Its Perspectives as Alternative of Liquid Fuels. In Liquid, Gaseous and Solid Biofuels (pp. 199-261).

Kujawska, A., Kujawski, J., Bryjak, M., \& Kujawski, W. (2015). ABE fermentation products recovery methods-A review. Renewable and Sustainable Energy Reviews, 48, 648-661.

Kumar, P., Barrett, D. M., Delwiche, M. J., \& Stroeve, P. (2009). Methods for Pretreatment of Lignocellulosic Biomass for Efficient Hydrolysis and Biofuel Production. Industrial \& Engineering Chemistry Research, 48(8), 3713-3729. 
Kumar, V., \& Jain, S. M. (2014). Plants and algae species: Promising renewable energy production source, 26(8), 679-692.

Kusdiana, D., \& Saka, S. (2004). Effects of water on biodiesel fuel production by supercritical methanol treatment. Bioresource Technology, 91(3), 289-95.

Laan, T., Litman, T. A., \& Steenblik, R. (2009). BIOFUELS - AT WHAT COST? Government support for ethanol and biodiesel in Canada: One of a series of reports addressing subsidies for biofuels in selected OECD countries. Winnipeg. (pp. 1-121).

Lackey, J.C.; Peppley, B.; Champagne, P.; Maier, A. (2015). Composition and uses of anaerobic digestion derived biogas from wastewater treatment facilities in NA. Waste Management \& Research, 33(8), 767-771.

Lapurta, M., Rodriguez-Fernandez, J.,Oliva, F. (2010). Determination of enthalpy of formation of methyl and ethyl esters of fatty acids. Chemistry and Physics of Lipids, 163, 172-181.

Lardon, L., Hélias, A., Sialve, B., Steyer, J. P., \& Bernard, O. (2009). Life-cycle assessment of biodiesel production from microalgae. Environmental Science and Technology, 43(17), 6475-6481.

Le Roy, D. G., \& Klein, K. K. (2012). The Policy Objectives of a Biofuel Industry in Canada: An Assessment. Agriculture, 2, 436-451.

Lee, J., Yoo, C., Jun, S., Ahn, C., \& Oh, H. (2010). Comparison of several methods for effective lipid extraction from microalgae. Bioresource Technology, 101(1), S75-S77.

Leite, G. B., \& Hallenbeck, P. C. (2012). Algae Oil. In Microbial Technologies in Advanced Biofuels Production (pp. 231-259). Boston, MA: Springer.

Liang, K., Zhang, Q., \& Cong, W. (2012). Enzyme-Assisted Aqueous Extraction of Lipid from Microalgae. Journal of Agricultural and Food Chemistry, 60(47), 11771-11776.

Linden, J. C., Moreira, A. R., \& Lenz, T. G. (1985). Acetone and Butanol. In M. Moo-Young (Ed.), Comprehensive Biotechnology (pp. 915-929). Oxford, New York, Toronto, Sydney, Frankfurt: Pergamon Press.

Liu, F., Li, W., Sun, Q., Zhu, L., Meng, X., Guo, Y.-H., \& Xiao, F.-S. (2011). Transesterification to Biodiesel with Superhydrophobic Porous Solid Base Catalysts. ChemSusChem, (4), 1059-1062.

Liu, J. (2013). Biodiesel Synthesis via Transesterification Reaction in Supercritical Methanol:a) A Kinetic Study, b) Biodiesel Synthesis Using Microalgae Oil [Master's Thesis]. Syracuse University.

Liu, J., Song, Y., \& Qiu, W. (2017). Oleaginous microalgae Nannochloropsis as a new model for biofuel production_Review ampamp; analysis. Renewable and Sustainable Energy Reviews, 72, 154-162.

Liu, W., Jiang, H., \& Yu, H. (2015). Thermochemical conversion of lignin to functional materials: a review and future directions. Green Chemistry, 17, 4888-4907.

Lundquist, T., Woertz, I., Quinn, N., \& Benemann, J. (2010). A Realistic Technology and 
Engineering Assessment of Algae Biofuel Production. Energy Biosciences Institute. San Luis Obispo. (pp. 1-178).

Ma, X. (2016). UTILIZATION OF WASTE RESOURCES FOR LOW-COST ALGAE-BASED BIOFUEL [Doctoral dissertation]. University of Minnesota.

Maddi, B., Panisko, E., Wietsma, T., Lemmon, T., Swita, M., Albrecht, K., \& Howe, D. (2016). Quantitative characterization of the aqueous fraction from hydrothermal liquefaction of algae. Biomass and Bioenergy, 93, 122-130.

Martin, N. (1998). Separating Azeotropic Mixtures. Retrieved from https://www.scribd.com/document/239403769/Separating-Azeotropic-Mixtures

Martinez, M. E., Sanchez, S., Jim Enez, J. M., El Yousfi, F., \& Munoz, L. (2000). Nitrogen and phosphorus removal from urban wastewater by the microalga Scenedesmus obliquus. Biosource Technology, (73), 263-272.

Marulanda, V. F., Anitescu, G., \& Tavlarides, L. L. (2010). Investigations on supercritical transesterification of chicken fat for biodiesel production from low-cost lipid feedstocks. The Journal of Supercritical Fluids, 54(1), 53-60.

Mason, T. J., \& Lorimer, J. P. (2002). Applied Sonochemistry. Weinheim, FRG: Wiley-VCH Verlag $\mathrm{GmbH} \& \mathrm{Co} . \mathrm{KGaA}$.

Mata, T. M., Martins, A. A., \& Caetano, N. S. (2010). Microalgae for biodiesel production and other applications: a review. Renew Sustainable Energy Review, 14, 217-232.

Mattarelli, E., Rinaldini, C., \& Savioli, T. (2015). Combustion Analysis of a Diesel Engine Running on Different Biodiesel Blends. Energies, 8(4), 3047-3057.

Maurice, M. L., Advisor: David DiBiasio, \& Co Advisor: Yan Lin. (2011). Factors Effecting Ethanol Fermentation Via Simultaneous Saccharification and Fermentation [Undergraduate report]. Worcester Massachusetts.

McGregor, D. (2004). Coming Full Circle: Indigenous Knowledge, Environment, and Our Future. American Indian Quarterly, 28(3/4), 385-410.

McMichael, A. J., Powles, J. W., Butler, C. D., \& Uauy, R. (2007). Food, livestock production, energy, climate change, and health. The Lancet, 370(9594), 1253-1263.

Merrett, M. J., Nimer, N. A., \& Dong, L. F. (1996). The utilization of bicarbonate ions by the marine microalga Nannochloropsis oculata (Droop) Hibberd. Plant, Cell and Environment, 19(4), 478-484.

Miao, X., \& Wu, Q. (2006). Biodiesel production from heterotrophic microalgal oil. Bioresource Technology, 97(6), 841-846.

Mills, G. A., \& Ecklund, E. E. (1987). ALCOHOLS AS COMPONENTS OF TRANSPORTATION FUELS 1. Annual Review Energy, 12, 47-80.

Min, M., Hu, B., Mohr, M. J., Shi, A., Ding, J., Sun, Y., ... Ruan, R. (2014). Swine ManureBased Pilot-Scale Algal Biomass Production System for Fuel Production and Wastewater Treatment-a Case Study. Applied Biochemistry and Biotechnology, 172, 1390-1406. 
Min, M., Wang, L., Li, Y., Mohr, M. J., Hu, B., Zhou, W., ... Ruan, R. (2011). Cultivating Chlorella sp. in a Pilot-Scale Photobioreactor Using Centrate Wastewater for Microalgae Biomass Production and Wastewater Nutrient Removal. Applied Biochemistry and Biotechnology, 165, 123-137.

Molina Grima, E., Acién Fernández, F. G., Camacho, F. G., \& Chisti, Y. (1999). Photobioreactors: light regime, mass transfer, and scaleup. Journal of Biotechnology, 70, 231-247.

Molina Grima, M. E., Belarbi, E. H., Acién Fernández, F. G., Robles Medina, A., \& Chisti, Y. (2003). Recovery of microalgal biomass and metabolites: process options and economics. Biotechnology Advances, 20(7-8), 491-515.

Monick, J. A. (1968). Alcohols their chemistry, properties and manufacture. New York: Reinhold.

Moo-Young, M. (Ed.). (1985). Comprehensive biotechnology : the principles, applications, and regulations of biotechnology in industry, agriculture, and medicine (3rd ed.). New York: Pergamon.

Moorhouse, J., \& Wolinetz, M. (2016). Biofuels in Canada: Tracking progress in tackling greenhouse gas emissions from transportation fuels. Burnaby. (pp. 1-29).

Morris, A. E., Fine, H. A., Geiger, G. H., \& Fine, H. A. (2011). Handbook on material and energy balance calculations in materials processing. John Wiley.

Mostafaei, M., Ghobadian, B., Barzegar, M., \& Banakar, A. (2015). Optimization of ultrasonic assisted continuous production of biodiesel using response surface methodology. Ultrasonics Sonochemistry, 27, 54-61.

Mu, D., Seager, T., Suresh Rao, P., \& Zhao, F. (2010). Comparative Life Cycle Assessment of Lignocellulosic Ethanol Production: Biochemical Versus Thermochemical Conversion. Environmental Management, (46), 565-578.

Murakami, M., \& Ikenouchi, M. (1997). The biological CO2 fixation and utilization project by rite (2) - Screening and breeding of microalgae with high capability in fixing CO2. Energy Conversion and Management, 38, S493-S497.

Naderloo, L., Javadikia, H., \& Mostafaei, M. (2017). Modeling the energy ratio and productivity of biodiesel with different reactor dimensions and ultrasonic power using ANFIS. Renewable and Sustainable Energy Reviews, 70, 56-64.

Nanaki, E. A., \& Koroneos, C. J. (2012). Comparative LCA of the use of biodiesel, diesel and gasoline for transportation. Journal of Cleaner Production, 20(1), 14-19.

National Energy Board. (2017). Canada's Energy Future 2016: Province and Territory Outlooks. Retrieved March 19, 2018, from https://www.neb-one.gc.ca/nrg/ntgrtd/ftr/2016pt/prvnctrrtrl-cmprsn-eng.html

Natural Resources Canada. (2015a). Biodiesel - Government Programs and Regulations. Retrieved January 18, 2018, from http://www.nrcan.gc.ca/energy/alternative-fuels/fuelfacts/biodiesel $/ 3515$ 
Natural Resources Canada. (2015b). Electricity facts. Retrieved March 9, 2018, from https://www.nrcan.gc.ca/energy/facts/electricity/20068

Natural Resources Canada. (2018). Biodiesel - Government Programs and Regulations | Natural Resources Canada. Retrieved March 18, 2018, from http://www.nrcan.gc.ca/energy/alternative-fuels/fuel-facts/biodiesel/3515

Neste. (2018). What is the difference between renewable diesel and traditional biodiesel - if any? Retrieved March 13, 2018, from https://www.neste.com/en/what-difference-betweenrenewable-diesel-and-traditional-biodiesel-if-any

Noureddini, H., Bandlamudi, S. R. P., \& Guthrie, E. A. (2009). A Novel Method for the Production of Biodiesel from the Whole Stillage-Extracted Corn Oil. Journal of the American Oil Chemists' Society, 86(1), 83-91.

Obasi, G. O. ., \& Tolba, M. K. (1992). Climate Change: First Assessment Report Overview and Policymaker Summaries. Geneva. (pp. 1-178).

Ogden, K. L. (2014). Algae as a Bio-Feedstock. Chemical Engineering Progress, 11(110), 6366.

Oil Sands Magazine. (2017). Oil Sands Magazine - Do you know where your oil comes from? Retrieved January 4, 2018, from http://www.oilsandsmagazine.com/news/2015/6/11/doyou-know-where-your-oil-comes-from

Olsson, J., Feng, X. M., Ascue, J., Gentili, F. G., Shabiimam, M. A., Nehrenheim, E., \& Thorin, E. (2014). Co-digestion of cultivated microalgae and sewage sludge from municipal waste water treatment. BIORESOURCE TECHNOLOGY, 171, 203-210.

Ontario Environmental Protection Act. GREENER DIESEL - RENEWABLE FUEL CONTENT REQUIREMENTS FOR PETROLEUM DIESEL FUEL, Reg. 97/14 Ontario Environmental Protection Act (2014). Government of Ontario.

Ontario Ministry of the Environment and Climate Change. (2016). Ontario 2016 Five Year Climate Action Change Plan. Toronto. (pp. 1-86).

Oreskes, N. (2004). Beyond the ivory tower. The scientific consensus on climate change. Science (New York, N.Y.), 306(5702), 1686.

Oreskes, N. (2018). Decoding the resistance to climate change, [Audio podcast]. Canadian Broadcast Corporation - Ideas.

Oswald, W. J. (1988). Micro-algae and waste-water treatment. In M. A. Borowitzka \& L. J. Borowitzka (Eds.), Micro-algal Biotechnology (pp. 305-328). Cambridge: Cambridge University Press.

Oswald, W. J., \& Benemann, J. R. (1977). A critical analysis of bioconversion with microalgae. In A. Mitsui, S. Miyachi, A. San Pietro, \& S. Tamura (Eds.), Biological solar energy conversion (pp. 379-396). New York: Oxford: Elsevier Science.

Oswald, W. J., \& Gotaas, H. B. (1957). Photosynthesis in Sewage Treatment. Transactions of the American Society of Civil Engineers, 122(1), 73-97. 
Oxford Instruments. (2018). Fatty Acid Composition of Triglycerides. Retrieved March 19, 2018, from https://www.oxford-instruments.com/industries-and-applications/agriculturefood-beverage/food-beverages/fatty-acid-composition-of-triglycerides

Parkin, G. F., \& Owen, W. F. (1986). FUNDAMENTALS OF ANAEROBIC DIGESTION OF WASTEWATER SLUDGES. Journal of Environmental Engineering, 112(5), 867-920.

Pasadakis, N., Gaganis, V., \& Foteinopoulos, C. (2006). Octane number prediction for gasoline blends. Fuel Processing Technology, 87, 505-509.

Patel, D., Kellici, S., \& Saha, B. (2014). Green Process Engineering as the Key to Future Processes. Processes, 2, 311-332.

Patil, P. D., Gude, V. G., Mannarswamy, A., Cooke, P., Nirmalakhandan, N., Lammers, P., \& Deng, S. (2012). Comparison of direct transesterification of algal biomass under supercritical methanol and microwave irradiation conditions. Fuel, 97, 822-831.

Patwardhan, A. W. (2003). Rotating Biological Contactors: A Review. Industrial and Engineering Chemistry Research, 42(10), 2035-2051.

Pegallapati, A. K., \& Frank, E. D. (2016). Energy use and greenhouse gas emissions from an algae fractionation process for producing renewable diesel. ALGAL, 18, 235-240.

Perry, R. H., Green, D. W., \& Maloney, J. O. (Eds.). (1997). Perry's Chemical Engineering Handbook (7th ed.). New York: McGraw-Hill.

Petrick, I., Dombrowski Michael Kröger, L., Beckert Thomas Kuchling, T., \& Kureti, S. (2013). Algae Biorefinery - Material and energy use of algae. Leipzig. (pp. 1-13).

Pfromm, P. H., Amanor-Boadu, V., \& Nelson, R. (2011). Sustainability of algae derived biodiesel: A mass balance approach. Bioresource Technology, 102, 1185-1193.

Pienkos, P. T., \& Darzins, A. (2009). The promise and challenges of microalgal-derived biofuels. Biofuels, Bioproducts and Biorefining, 3, 431-440.

Pinnarat, T., \& Savage, P. E. (2008). Assessment of Noncatalytic Biodiesel Synthesis Using Supercritical Reaction Conditions. Industrial \& Engineering Chemistry Research, 47(18), 6801-6808.

Pittman, J. K., Dean, A. P., \& Osundeko, O. (2011). The potential of sustainable algal biofuel production using wastewater resources. Bioresource Technology, 102, 17-25.

Pond Technologies Inc. (2017). Energy and Algae. Retrieved January 7, 2018, from http://pondtechnologiesinc.com/technology/energy-and-algae/

Poux, M. (2014a). 4th International Congress on Green Process Engineering (GPE2014; Sevilla, Spain, April 7-10,2014). Green Processing and Synthesis, 3(5), 309-310.

Poux, M. (2014b). 4th International Congress on Green Process Engineering (GPE2014) continued. Green Processing and Synthesis, 3(6), 399-399.

Premium Pulman PVT Ltd. (2017). Air Swept Pulverizers. Retrieved December 11, 2017, from http://www.premiumpulverisers.com/a_mill.htm 
Qin, D., Plattner, G. K., Tignor, M., Allen, S. K., Boschung, J., Nauels, A., ... Midgley, P. M. (2013). IPCC 2013 - Summary for Policymakers. Cambridge \& New York. (pp. 1-28).

Quinn, J. C., \& Davis, R. (2015). The potentials and challenges of algae based biofuels: A review of the techno-economic, life cycle, and resource assessment modeling. Bioresource Technology, 184, 444-452.

Quiroz-Castañeda, R. E., \& Folch-Mallol, J. L. (2013). Hydrolysis of Biomass Mediated by Cellulases for the Production of Sugars. In A. Chandel (Ed.), Sustainable Degredation of Lignocellulosic Biomass - Techniques, Application and Commercialization (Vol. 3, pp. 119-155). InTech.

Qureshi, N., Li, X., Hughes, S., Saha, B. C., \& Cotta, M. A. (2006). Butanol Production from Corn Fiber Xylan Using Clostridium acetobutylicum. Biotechnology Progress, 22(3), 673680.

Radmer, R. J. (1996). Algal Diversity and Commercial Algal Products. Source: BioScience Marine Biotechnology, 46(4), 263-270.

Ragauskas, A. J., Williams, C. K., Davison, B. H., Britovsek, G., Cairney, J., Eckert, C. A., ... Tschaplinski, T. (2005). Production of Liquid Alkanes by Aqueous-Phase Processing of Biomass-Derived Carbohydrates. Science, 308(5727), 1446-1450.

Rakopoulos, D. C., Rakopoulos, C. D., Papagiannakis, R. G., \& Kyritsis, D. C. (2011). Combustion heat release analysis of ethanol or n-butanol diesel fuel blends in heavy-duty DI diesel engine. Fuel, 90, 1855-1867.

Rance Bare, W. F., Jones, N. B., \& Middlebrooks, E. J. (1975). Algae Removal Using Dissolved Air Flotation. Source Journal (Water Pollution Control Federation), 47(1), 153-169.

Ratledge, C. (2004). Fatty acid biosynthesis in microorganisms being used for Single Cell Oil production. Biochimi, 86(11), 807-815.

Ribeiro, R. L. L., Vargas, J. V. C., Mariano, A. B., Ordonez, J. C., \& Vargas, J. V. C. (2017). The experimental validation of a large-scale compact tubular microalgae photobioreactor model. International Journal of Energy Research, 41, 2221-2235.

Romero-García, J. M., Niño, L., Martínez-Patiño, C., Álvarez, C., Castro, E., \& Negro, M. J. (2014). Biorefinery based on olive biomass. State of the art and future trends. Bioresource Technology, 159, 421-432.

Rosello Sastre, R., Csögör, Z., Perner-Nochta, I., Fleck-Schneider, P., \& Posten, C. (2007). Scale-down of microalgae cultivations in tubular photo-bioreactors-A conceptual approach. Journal of Biotechnology, 132(2), 127-133.

Ross, C. C., Brandon, P. E., Smith, M., \& Valentine, E. I. T. G. E. (2000). RETHINKING DISSOLVED AIR FLOTATION (DAF) DESIGN FOR INDUSTRIAL PRETREATMENT. Atlanta. (pp.1-14).

Saini, J. K., Saini, R., \& Tewari, L. (2015). Lignocellulosic agriculture wastes as biomass feedstocks for second-generation bioethanol production: concepts and recent developments. Biotechnology, 5(4), 337-353. 
Sakai, N., Sakamoto, Y., Kishimoto, N., Chihara, M., \& Karube, I. (1995). Chlorella strains from hot springs tolerant to high temperature and high CO2. Energy Conversion and Management, 36(6-9), 693-696.

Salim, S., Bosma, R., Vermuë, M. H., \& Wijffels, R. H. (2011). Harvesting of microalgae by bio-flocculation. Journal of Applied Phycology, 23(5), 849-855.

Sander, K., \& Murthy, G. S. (2010). Life cycle analysis of algae biodiesel. International Journal of Life Cycle Assessment, 15(7), 704-714.

Sanhueza, P. A., Torreblanca, M. A., Diaz-Robles, L. A., Schiappacasse, L. N., Silva, M. P., \& Astete, T. D. (2009). Particulate Air Pollution and Health Effects for Cardiovascular and Respiratory Causes in Temuco, Chile: A Wood-Smoke-Polluted Urban Area. Journal of the Air \& Waste Management Association, 59(12), 1481-1488.

Sazhin, S. S., Al Qubeissi, M., Kolodnytska, R., Elwardany, A. E., Nasiri, R., \& Heikal, M. R. (2014). Modelling of biodiesel fuel droplet heating and evaporation. Fuel, 115, 559-572.

Scavia, D., David Allan, J., Arend, K. K., Bartell, S., Beletsky, D., Bosch, N. S., ... Zhou, Y. (2014). Assessing and addressing the re-eutrophication of Lake Erie: Central basin hypoxia. Journal of Great Lakes Research, 40(2), 226-246.

Schädlich, K., Schug, P., Fabri, J., Dabelstein, W., \& Reglitzky, A. (2003). Octane Enhancers. In Ullmann's Encyclopedia of Industrial Chemistry. Weinheim, Germany: Wiley-VCH Verlag $\mathrm{GmbH} \& \mathrm{Co}$. KGaA.

Schenk, P. M., Thomas-Hall, S. R., Stephens, E., Marx, U. C., Mussgnug, J. H., Posten, C., ... Hankamer, B. (2008). Second Generation Biofuels: High-Efficiency Microalgae for Biodiesel Production. BioEnergy Research, 1(1), 20-43.

Sen, B., Tahir, M., Sonmez, F., Turan Kocer, M. A., \& Canpolat, O. (2013). Relationship of Algae to Water Pollution and Waste Water Treatment. In Water Treatment (1st ed., pp. 335-354). Elasig Turkey: InTech.

Seraj, H. (2014). ASSESSING THE COMMERCIALIZATION POTENTIAL OF ALGAL JET FUEL FROM A LIFECYCLE ASSESSMENT APPROACH [Master's Thesis]. Ryerson University.

Sharara, Mahmoud; Clausen, Edgar; Carrier, D. (2012). An Overview of Biorefinery Technology. In C. V. Stevens (Ed.), Biorefinery Co-Products (p. 1). Ghent: Wiley.

Sheehan, J., Aden, A., Paustian, K., Killian, K., Brenner, J., Walsh, M., \& Nelson, R. (2003). Energy and Environmental Aspects of Using Corn Stover for Fuel Ethanol. Journal of Industrial Ecology, 7(3-4), 117-146.

Sheehan, J., Dunahay, T., Benemann, J., \& Roessler, P. (1998). Look Back at the U.S. Department of Energy's Aquatic Species Program: Biodiesel from Algae; Close-Out Report. Golden, CO. (pp. 1-296).

Shelef, G., Sukenik, A., \& Green, M. (1984). Microalgae harvesting and processing: a literature review. Golden, CO. (pp. 1-71). 
Shell Global. (2017). Hydrotreating. Retrieved January 18, 2018, from https://www.shell.com/business-customers/global-solutions/refinery-technologylicensing/hydrotreating.html

Shen, Y., Linville, J. L., Anne, P., Ignacio-De Leon, A., Schoene, R. P., \& Urgun-Demirtas, M. (2016). Towards a sustainable paradigm of waste-to-energy process: Enhanced anaerobic digestion of sludge with woody biochar. Journal of Cleaner Production, 135, 1054-1064.

Shen, Y., Yang, T., Zhu, W., \& Zhao, Y. (2017). Wastewater treatment and biofuel production through attached culture of Chlorella vulgaris in a porous substratum biofilm reactor. Journal of Applied Physiology, 29, 833-841.

Shen, Y., Yuan, W., Pei, Z. J., Wu, Q., \& Mao, E. (2009). Microalgae Mass Production Methods. Transactions of the ASABE, 52(4), 1275-1287.

Silva, C., Soliman, E., Cameron, G., Fabiano, L. A., Seider, W. D., Dunlop, E. H., \& Coaldrake, A. K. (2014). Commercial-Scale Biodiesel Production from Algae. Industrial \& Engineering Chemistry Research, 53(13), 5311-5324.

Sims, R. E. H., Mabee, W., Saddler, J. N., \& Taylor, M. (2010). An overview of second generation biofuel technologies. Bioresource Technology, 101(6), 1570-1580.

Sinden, G., Callahan, W., Fava, J. A., King, H., Wickwire, S., \& Sottong, J. (2010). GHG Protocol: Product Life Cycle Accounting Reporting Standard. World Resources Institute \& WBCSD. (pp. 1-146).

Singh, A., Nigam, P. S., \& Murphy, J. D. (2011). Renewable fuels from algae: An answer to debatable land based fuels. Bioresource Technology, 102(1), 10-16.

Singh, M., Shukla, R., \& Das, K. (2013). Chapter 6: Harvesting of Microalgal Biomass. In F. Bux (Ed.), Biotechnological Applications of Microalgae (1st ed., p. 255). New York: CRC Press.

Singh, R. N. (2012). Development of suitable photobioreactor for algae production - A review. Renew Sustainable Energy Review, 16(4), 2347-2353.

Singh, U. B., \& Ahluwalia, A. S. (2013). Microalgae: A promising tool for carbon sequestration. Mitigation and Adaptation Strategies for Global Change, 18(1), 73-95.

SME China Mining Equipment. (2017). Ball Mill. Retrieved December 11, 2017, from http://sighri.in/products/ball-mill.html

Smerkowska, B. (2011). Biobutanol - Production and Application in Diesel Engines. CHEMIK, 65(6), 549-556.

Soeder, C. (1986). An historical outline of applied algology. In A. Richmond (Ed.), Handbook for Microalgal Mass Culture (pp. 25-41). Boca Raton, FL.: CRC Press.

Spier, M. R., Porto De Souza Vandenberghe, L., Bianchi, A., Medeiros, P., \& Soccol, C. R. (2011). APPLICATION OF DIFFERENT TYPES OF BIOREACTORS IN BIOPROCESSES. In P. G. Antolli \& Z. Liu (Eds.), Bioreactors: Design, Properties and Applications (pp. 55-90). Nova Science Publishers Inc. 
Spirinckx, C., \& Ceuterick, D. (1996). Biodiesel and Fossil Diesel Fuel: Comparative Life Cycle Assessment. International Journal of LCA, 1(3), 127-132.

Spoehr, H. A., \& Milner, H. W. (1949). THE CHEMICAL COMPOSITION OF CHLORELLA; EFFECT OF ENVIRONMENTAL CONDITIONS. Plant Physiology, 24(1), 120-49.

Stanbury, P. F., Whitaker, A., \& Hall, S. J. (2016). Principles of fermentation technology (3rd ed.). Butterworth-Heinemann.

Statistics Canada. (2015). Fuel used for road motor vehicles by province. Retrieved from http://www.statcan.gc.ca/tables-tableaux/sum-som/101/cst01/trade37b-eng.htm

Stephenson, A. L., Kazamia, E., Dennis, J. S., Howe, C. J., Scott, S. A., \& Smith, A. G. (2010). Life-Cycle Assessment of Potential Algal Biodiesel Production in the United Kingdom: A Comparison of Raceways and Air-Lift Tubular Bioreactors. Energy Fuels, 24, 4062-4077.

Subhadra, B., \& Grinson, G. (2011). Algal biorefinery-based industry: an approach to address fuel and food insecurity for a carbon-smart world. Journal of the Science of Food and Agriculture, 91(1), 2-13.

Suganya, T., Varman, M., Masjuki, H. H., \& Renganathan, S. (2016). Macroalgae and microalgae as a potential source for commercial applications along with biofuels production: A biorefinery approach. Renewable and Sustainable Energy Reviews, 55, 909941.

Suslick, K. S. (1989). The Chemical Effects of Ultrasound. Scientific American, 260, 80-87.

Syed, K. (2012). Development of Novel Clostridial Fusants For Enhanced Biobutanol Production [Master's Thesis]. Ryerson University.

Szwaja, S., \& Naber, J. D. (2009). Combustion of n-butanol in a spark-ignition IC engine. Fuel, $89,1573-1582$.

Taherzadeh, M. J., \& Karimi, K. (2007). ENZYME-BASED HYDROLYSIS PROCESSES FOR ETHANOL FROM LIGNOCELLULOSIC MATERIALS: A REVIEW. BioResources, 2(4), 707-738.

Tashiro, Y., Yoshida, T., Noguchi, T., \& Sonomoto, K. (2013). Recent advances and future prospects for increased butanol production by acetone-butanol-ethanol fermentation. Engineering in Life Sciences, 13(5), 432-445.

Tatum, C. (2012). Using the GREET Model to Analyze Algae as a feedstock for biodiesel production [Master's thesis]. University of Tennessee at Chattanooga.

Taylor, A., Raynolds, M., \& Contact, M. H. (2005). Bio-product Market Development Strategy: A Scoping Study on Federal Policies for Creating Market Demand for Bio-products in Canada. Calgary. (pp. 1-45).

Tchobanoglous, G., Burton, F. L., \& Stensel, H. D. (1991). Wastewater Engineering: Treatment and reuse. (Metcalf \& Eddy Inc, Ed.) (4th ed.). New York: McGraw Hill.

Teixeira, M. R., \& Rosa, M. J. (2007). Comparing dissolved air flotation and conventional sedimentation to remove cyanobacterial cells of Microcystis aeruginosa Part II. The effect 
of water background organics. Separation and Purification Technology, 53, 126-134.

The DOW Chemical Company. (1997). DOWTHERM A Heat Transfer Fluid - product technical data. Retrieved from https://www.dow.com/enus/products/DOWTHERMSyntheticOrganicFluids

The Dupps Company. (2017). Evaporator System Basics. Germantown. Retrieved from http://www.dupps.com/Evaporator Basics.pdf

The Government of Canada. Canada Foundation for Sustainable Development Technology Act/ Loi sur la Foundation du Canada pour l'appui technologique developpement durable, Pub. L. No. S.C. 2001, C 23 (2001).

The Government of Canada. Federal Sustainable Develoment Act/ Loi federale sur le developpement durable, Pub. L. No. S.C. 2008, c. 33 (2008).

The Government of Ontario. (2018). Ontario's Cleantech Strategy. Retrieved March 7, 2018, from https://www.ontario.ca/page/ontarios-cleantech-strategy

Thinkstep GaBi. (2017). LCA and introduction to GaBi - Part 1, Chapter 6: Understanding Flows. Retrieved January 22, 2018, from http://www.gabisoftware.com/international/support/gabi-learning-center/gabi-6-learning-center/part-1-lcaand-introduction-to-gabi/

Thinkstep representative. (2017). Private conversation with Cheryl Carbon. Boston: Thinkstep $\mathrm{GaBi}$.

Tian, Y., \& Zhao, C. Y. (2013). A review of solar collectors and thermal energy storage in solar thermal applications. Applied Energy, 104, 538-553.

Tonietto, E. A., Lombardi, A. T., Vieira, A. A. H., Parrish, C. C., \& Choueri, R. B. (2014). Cylindrospermopsis raciborskii (Cyanobacteria) exudates: Chemical characterization and complexation capacity for $\mathrm{Cu}, \mathrm{Zn}, \mathrm{Cd}$ and $\mathrm{Pb}$. Water Research, 49, 381-390.

Toptionlab. (2017). Spray Dryer (Lab). Retrieved January 13, 2018, from http://www.toptionlab.com/product/Lab-Spray-Dryer.html

Torri, S. I., Corrêa, R. S., \& Renella, G. (2014). Soil Carbon Sequestration Resulting from Biosolids Application. Applied and Environmental Soil Science, 2014, 1-9.

Toulouse INP (Universite). (2018). International Congress on Green Process Engineering. Toulouse, Mont Tremblant, Venice, Kuala Lumpur, Seville.

Towers, L. (2016). Grinding Equipment for Aquatic Feeds. Retrieved December 11, 2017, from https://thefishsite.com/articles/grinding-equipment-for-aquatic-feeds

Travieso, L., Hall, D. ., Rao, K. ., Benítez, F., Sánchez, E., \& Borja, R. (2001). A helical tubular photobioreactor producing Spirulina in a semicontinuous mode. International Biodeterioration \& Biodegradation, 47(3), 151-155.

U.S. Energy Information Administration. (2017). What is U.S. electricity generation by energy source? Retrieved March 9, 2018, from https://www.eia.gov/tools/faqs/faq.php?id=427\&t=3 
U.S. Securities and Exchange Commission. (2005). Summary of Koch Industries history. Retrieved March 18, 2018, from https://www.sec.gov/Archives/edgar/data/41077/000119312505225697/dex993.htm

United States of America Argonne National Laboratory. (2017). GREET 2017. Argonne National Laboratory.

United States of America Centrifuge Systems. (2017). MAC 203 Disc Bowl Centrifuge - US Centrifuge Systems. Retrieved November 21, 2017, from https://www.uscentrifuge.com/centrifuges/disc-centrifuges/mac-203-disc-bowl-centrifuge/

United States of America Department of Energy. (2015). Quadrennial Energy Review - Energy Transmission, Storage and Distribution Infrastructure: Appendix A - Liquid Fuels. Washington. (pp. 1-45).

United States of America Department of Energy. (2017). 2017 Project Peer Review - U.S. D.O.E. Bioenergy Technologies Office. (pp. 1-704)

University of Western Ontario. (2018). Green Process Engineering Program - Western University. Retrieved March 6, 2018, from https://www.eng.uwo.ca/undergraduate/programs/GreenProcess.html

Usui, N., \& Ikenouchi, M. (1997). The biological CO2 fixation and utilization project by RITE(1) - Highly-effective photobioreactor system - Energy Conversion and Management, 38, S487-S492.

Vandamme, D. (2013). Flocculation based harvesting processes for microalgae biomass production [Doctoral dissertation]. KU Leuven.

Vasudevan, P. T., \& Briggs, M. (2008). Biodiesel production — current state of the art and challenges. Industrial Microbiology and Biotechnology, 35, 421-430.

Vobis LLC. (2017). Types of Evaporators - Vobis, LLC. Retrieved February 1, 2018, from http://www.vobisllc.com/types-of-evaporators/

Vogelesang, H. (2008). An introduction to energy consumption in pumps. World Pumps, 2008(496), 28-31.

Vonshak, A., \& Richmond, A. (1988). Mass Production of the Blue-green Alga Spirulina: An Overview*. Biomass, 15, 233-247.

Wang, B., Lan, C. Q., \& Horsman, M. (2012). Closed photobioreactors for production of microalgal biomasses. Biotechnology Advances, 30(4), 904-912.

Wang, B., Li, Y., Wu, N., \& Lan, C. Q. (2008). CO2 bio-mitigation using microalgae. Applied Microbiology and Biotechnology, 79(5), 707-718.

Wang, L., Min, M., Li, Y., Chen, P., Chen, Y., Liu, Y., ... Ruan, R. (2010). Cultivation of Green Algae Chlorella sp. in Different Wastewaters from Municipal Wastewater Treatment Plant. Applied Biochemistry and Biotechnology, 162(4), 1174-1186.

Watson, S. B., Miller, C., Arhonditsis, G., Boyer, G. L., Carmichael, W., Charlton, M. N., ... Wilhelm, S. W. (2016). The re-eutrophication of Lake Erie: Harmful algal blooms and 
hypoxia. Harmful Algae, 56, 44-66.

Weaver, A. (2017). I could not look my children in the face and say I did nothing, [Audio podcast]. CBC Radio - The Sunday Edition.

Weidema, B. (2000). Avoiding Co-Product Allocation in Life-Cycle Assessment. Journal of Industrial Ecology, 4(3), 11-33.

Weidema, B., Wenzel, H., Petersen, C., \& Hansen, K. (2004). The Product, Functional Unit and Reference Flows in LCA Introduction to the series. Copenhagen. (pp. 1-47).

Wiens, J., Fargione, J., \& Hill, J. (2011). Biofuels and biodiversity. Ecological Applications, $21(4), 1085-1095$.

Wiley, P. E., Campbell, J. E., \& McKuin, B. (2011). Production of Biodiesel and Biogas from Algae: A Review of Process Train Options. Water Environment Research, 83(4), 366-338.

Williams, P. J. le B., \& Laurens, L. M. L. (2010). Microalgae as biodiesel and biomass feedstocks: Review and analysis of the biochemistry, energetics and economics. Energy \& Environmental Science, 3, 554-590.

Willson, B. D., Turner, C. W., Babbitt, G. R., Letvin, P. A., \& Wickrmasinghe, S. R. (2009). Permeable membranes in film photobioreactors. U.S. Patent No. 20090305389, United Stated of America.

Wittcoff, H., Reuben, B. G., \& Plotkin, J. S. (2004). Industrial organic chemicals. (2nd ed.). Wiley-Interscience.

Wittcoff, H., Reuben, B. G., \& Plotkin, J. S. (2013). Industrial organic chemicals (3rd ed.). John Wiley \& Sons, Inc.

Woertz, I., Feffer, A., Lundquist, T., \& Nelson, Y. (2009). Algae Grown on Dairy and Municipal Wastewater for Simultaneous Nutrient Removal and Lipid Production for Biofuel Feedstock. Journal of Environmental Engineering, 135(11), 1115-1122.

Wu, Xiaodan; Ruan, Rongsheng; Du, Zhenyi; Liu, Y. (2014). Advances in Biofuel Production Algae and Aquatic Plants. (B. Gikonyo, Ed.). Toronto: Apple Academic Press; CRC Press.

Wu, C., Xiao, Y., Lin, W., Zhu, J., De la Hoz Siegler, H., Zong, M., \& Rong, J. (2017). Surfactants assist in lipid extraction from wet Nannochloropsis sp. Bioresource Technology, 243, 793-799.

Wu, M., Want, M., Liu, J., \& Huo, H. (2007). Life-Cycle Assessment of Corn-Based Butanol as a Potential Transportation Fuel. Argonne Illinois. (pp. 1-59).

Xu, H., Miao, X., \& Wu, Q. (2006). High quality biodiesel production from a microalga Chlorella protothecoides by heterotrophic growth in fermenters. Journal of Biotechnology, 126(4), 499-507.

Yang, F., Xiang, W., Fan, J., Wu, H., Li, T., \& Long, L. (2016). High pH-induced flocculation of marine Chlorella sp. for biofuel production. Journal of Applied Phycology, 28(2), 747-756.

Yen, H. W., \& Wang, Y.-C. (2013). The enhancement of butanol production by in situ butanol 
removal using biodiesel extraction in the fermentation of $\mathrm{ABE}$ (acetone-butanol-ethanol). Bioresource Technology, 145, 224-228.

Yoo, C., Jun, S. Y., Lee, J. Y., Ahn, C.-Y., \& Oh, H.-M. (2009). Selection of microalgae for lipid production under high levels carbon dioxide. Bioresource Technology, 101(1), S71-S74.

Yuan, J., Kendall, A., \& Zhang, Y. (2015). Mass balance and life cycle assessment of biodiesel from microalgae incorporated with nutrient recycling options and technology uncertainties. GCB Bioenergy, 7, 1245-1259.

Yüksel, F., \& Yüksel, B. (2004). The use of ethanol-gasoline blend as a fuel in an SI engine. Renewable Energy, 29(7), 1181-1191.

Yun, Y., Lee, S. B., Park, J. M., Lee, C., \& Yang, J. (1997). Carbon Dioxide Fixation by Algal Cultivation Using Wastewater Nutrients. Journal of Chemical Technology and Biotechnology, 69(69), 451-455.

Zaimes, G. G., \& Khanna, V. (2013). Environmental sustainability of emerging algal biofuels: A comparative life cycle evaluation of algal biodiesel and renewable diesel. Environmental Progress \& Sustainable Energy, 32(4), 926-936.

Zeevalkink, J. A., Kelderman, P., Visser, D. C., \& Boelhouwer, C. (1979). Physical mass transfer in a rotating disc gas-liquid contactor. Water Research, 13(9), 913-919.

Zeng, D., Li, R., Jin, T., \& Fang, T. (2014). Calculating the Thermodynamic Characteristics and Chemical Equilibrium of the Stepwise Transesterification of Triolein Using Supercritical Lower Alcohols. Industrial and Engineering Chemistry Research, (53), 7209-7216.

Zheng, H., Yin, J., Gao, Z., Huang, H., Ji, X., \& Dou, C. (2011). Disruption of Chlorella vulgaris Cells for the Release of Biodiesel-Producing Lipids: A Comparison of Grinding, Ultrasonication, Bead Milling, Enzymatic Lysis, and Microwaves. Applied Biochemistry and Biotechnology, 164(7), 1215-1224.

Zhou, N., Zhang, Y., Wu, X., Gong, X., \& Wang, Q. (2011). Hydrolysis of Chlorella biomass for fermentable sugars in the presence of $\mathrm{HCl}$ and $\mathrm{MgCl}$. Bioresource Technology, 102, 1015810161.

Zhu, L. (2015). Biorefinery as a promising approach to promote microalgae industry: An innovative framework. Renewable and Sustainable Energy Reviews, 41, 1376-1384. 